\title{
Bail-ins and Bail-outs: Incentives, Connectivity, and Systemic Stability
}

\author{
Benjamin Bernard, Agostino Capponi, and Joseph E. Stiglitz*
}

This paper endogenizes intervention in financial crises as the strategic negotiation between a regulator and creditors of distressed banks. Incentives for banks to contribute to a voluntary bail-in arise from their exposure to credit and price-mediated contagion. In equilibrium, a bail-in is possible only if the regulator's threat to not bail out insolvent banks is credible. Contrary to models without intervention or government bailouts only, sparse networks are beneficial in our model for two main reasons: they improve the credibility of the regulator's no-bailout threat for large shocks and they reduce free-riding incentives among bail-in contributors when the threat is credible.

Financial institutions are linked to each other via bilateral contractual obligations and are thus exposed to counterparty risk of their obligors. If one institution defaults on its liabilities, it affects the solvency of its creditors. Since the creditors are also borrowers, they may not be able to repay what they owe and default themselves - problems in one financial institution spread to others in what is known as financial contagion. Large shocks can trigger a cascade of defaults, which impose negative externalities on the economy. The extent of these cascades - the magnitude of the systemic risk - depends on the nature of the linkages, i.e., the structure of the financial system. In the 2008 crisis, it became apparent that the financial system had evolved in a way which enhanced its ability to absorb small shocks but made it more fragile in the face of a large shock. While a few studies called attention to these issues before the crisis, it was only after the crisis that the impact of the network structure on systemic risk became a major object of analysis 1 Most of the existing studies analyze the systemic risk implications of a default cascade, taking into account the network structure, asset liquidation costs, and different forms of inefficiencies that

\footnotetext{
*Bernard: Department of Economics, National Taiwan University, No. 1, Sec. 4, Roosevelt Rd, Da'an District, Taipei City, 10617 Taiwan; benbernard@ntu.edu.tw. Capponi: Department of Industrial Engineering and Operations Research, Columbia University, 500 W 120th St, Mudd Hall 535-G, New York, NY 10027; ac3827@columbia.edu. Stiglitz: Columbia Business School, Columbia University, 3022 Broadway, Uris Hall 212, New York, NY 10027; jes322@gsb.columbia.edu We are grateful to George Pennacchi (discussant), Yiming Ma (discussant), Asuman Ozdaglar, Alireza Tahbaz-Salehi (discussant), Darrell Duffie, Jakša Cvitanić, Matt Elliott, Douglas Gale, Matthew Jackson, Piero Gottardi, and Felix Corell for interesting discussions and perceptive comments. We would also like to thank the seminar participants of the Laboratory for Information and Decision Systems at the Massachusetts Institute of Technology, the Cambridge Finance Seminar series, the London School of Economics, Stanford University, New York University, the Fields Institute, the third annual conference on Network Science and Economics, the Columbia Conference on Financial Networks: Big Risks, Macroeconomic Externalities, and Policy Commitment Devices, the 2018 SFS Cavalcade North America, the 2019 American Finance Association meeting, and the 2018 North American Summer Meeting of the Econometric society for their valuable feedback. The research of Agostino Capponi is supported by a NSFCMMI: 1752326 CAREER grant. Benjamin Bernard acknowledges financial support from grant P2SKP1_171737 by the Swiss National Science Foundation. Joseph Stiglitz acknowledges the support of the Columbia Business School and of the grant on Financial Stability from the Institute for New Economic Thinking.

${ }^{1}$ Most notably, Allen and Gale (2000) and Greenwald and Stiglitz (2003). See also Boissay (2006), Castiglionesi 2007), May, Levin and Sugihara (2008), and Nier et al. (2007). One of the reasons for the limited study is the scarce availability of data on interbank linkages.
} 
arise at default. Many of these models, however, do not account for the possibility of intervention to stop the cascade. There is either no rescue of insolvent banks or the regulator (or central bank or other government institution) intervenes by following an exogenously specified protocol. The goal of our paper is to endogenize the intervention mechanism as the equilibrium outcome of the strategic interaction between regulator and financial institutions. We show that endogenizing the intervention plan affects the socially desirable network structure.

The most common default resolution procedure during the 2007-2009 financial crisis was the bailout $:^{2}$ In a bailout, the government injects liquidity to help distressed banks service their debt, effectively transferring liabilities from the private sector to the public sector. For example, the U.S. Treasury Department's Troubled Asset Relief Program (TARP) authorized expenditures of $\$ 700$ billion to prevent fire sale losses. (See also Duffie (2010) for a related discussion.) Some governments, such as Germany's, have called for private-sector participation through bail-ins, in which creditors write down their interbank claims against troubled banks $3^{3}$ Bail-ins effectively amount to a transfer of liabilities within the private sector, which places the burden of losses on creditors as opposed to taxpayers. A prominent example of a bail-in is the consortium organized by the Federal Reserve Bank of New York to rescue the hedge fund Long-Term Capital Management 4 In our paper, we will also consider assisted bail-ins, in which the regulator provides some liquidity assistance to incentivize the formation of a bail-in.

We model the provision of liquidity assistance as a three-stage sequential game between regulator and the banks. In the first stage, the regulator proposes an assisted bail-in allocation, which specifies the contributions by each solvent bank, as well as the additional liquidity injections that he will provide to each bank. In the second stage, each bank decides whether or not to participate in the proposed rescue. If all designated participants accept, the game ends with the proposed rescue consortium. Otherwise, the game proceeds to the third stage, in which the regulator chooses among three options: (i) use taxpayer money to contribute the debt amount that was supposed to be forgiven by the banks which rejected the proposal, (ii) abandon the bail-in coordination and resort to a public bailout, or (iii) avoid any intervention. After transfers are made, the banks' liabilities are cleared simultaneously in the spirit of Eisenberg and Noe (2001), possibly leading to a default cascade if the outcome of the negotiation leaves some banks insolvent.

Financial contagion in our model occurs through price-mediated contagion and credit contagion, the two most prominent channels of contagion identified by historical events. 5 First, distressed

\footnotetext{
${ }^{2}$ The Bush administration bailed out large financial institutions (AIG insurance, Bank of America, Bear Stearns and Citigroup) and government sponsored entities (Fannie Mae, Freddie Mac) at the heart of the crisis. The European Commission intervened to bail out financial institutions in Greece and Spain.

${ }^{3}$ In spite of such calls and the design of instruments to make private-sector participation automatic, there have been few successful bail-ins. Automatic participation is implemented through the use of "bail-inable debt" such as contingent-convertible bonds in order to reduce the banks' credit risk. The focus of this paper is on the welfare impact of default resolution policies after these risk-mitigating instruments have already been used.

${ }^{4}$ Long-Term Capital Portfolio collapsed in the late 1990s. On September 23, 1998, a recapitalization plan of $\$ 3.6$ billion was coordinated under the supervision of the Federal Reserve Bank of New York. A total of fourteen banks agreed to participate and two banks (Bear Stearns and Lehman Brothers) rejected the proposal.

${ }^{5}$ Quoting Greenspan (1998): "It was the judgment of officials at the Federal Reserve Bank of New York, who were monitoring the situation on an ongoing basis, that the act of unwinding LTCM's portfolio in a forced liquidation would not only have a significant distorting impact on market prices but also in the process could produce large losses, or worse, for a number of
} 
banks may have to liquidate some of their asset holdings in order to fulfill their obligations. Doing so over a short time period will have a knock-on effect on the asset price, causing losses to other holders of the asset. If several banks liquidate the asset simultaneously, this leads to a self-reinforcing mechanism, where a price drop forces banks to sell more in order to raise the needed liquidity, causing further price drops, and so on. Second, if a distressed bank defaults on its obligations, it will transmit losses to its creditors. Bankruptcy imposes deadweight losses, which are amplified by the feedback loops among defaulting banks.

We investigate the structure of default resolution plans that arise in equilibrium when the regulator cannot credibly commit to an ex-post suboptimal resolution policy. The lack of commitment power has important consequences for the regulator's negotiation power: if banks are aware that without their participation, the regulator prefers a bailout over no intervention, then they have no incentive to participate in any assisted bail-in the regulator proposes. We say that, in this case, the regulator's no-intervention threat fails to be credible ${ }^{6}$ Only if the threat is credible can an assisted bail-in be organized in equilibrium. Individually, a bank is willing to contribute up to the maximum it would lose in a default cascade. However, because losses are amplified as the shock propagates through the system, losses exceed the required ex-ante contributions. Therefore, it is not necessary that every bank contributes and banks have an incentive to free-ride on the contributions of others. In the equilibrium bail-in, the set of contributing banks minimizes free-riding incentives by consisting of the banks with the largest exposure to contagion. It thus follows that banks are willing to contribute more in sparser networks: because losses are more concentrated, the benefits of a bail-in are more targeted to the contributors than in more diversified networks, thereby reducing free-riding incentives.

A key determinant of the equilibrium outcome is the credibility of the regulator's no-intervention threat. We show that it is credible if and only if the losses generated by the regulator's inactionequal to the amplification of the shock as it propagates through the network - does not exceed a given threshold. Whether the shock amplification increases with the size of the initial shock faster than the threshold rises depends on asset illiquidity and on the network structure. We identify a variable, which we call the total throughput of defaulting banks, as a sufficient statistic for the dependence of the credibility on the network structure, conditional on the banks' levels of solvency and their total claims on solvent banks. The total throughput measures the rate of spillover losses transmitted to the solvent banks in the system. Conditional on the banks' solvency, the total throughput depends only on the network structure and not the banks' balance sheet quantities, making it a particularly convenient measure to compare different networks' potential to propagate losses. We demonstrate that the throughput increases as the connectivity of defaulting banks increases. As a result, in sparsely connected networks, the regulator's threat may not be credible for small shocks, but the credibility improves as the shock grows larger. Because the total

creditors and counterparties, and for other market participants who were not directly involved with LTCM. In that environment, it was the FRBNY's judgment that it was to the advantage of all parties-including the creditors and other market participantsto engender if at all possible an orderly resolution rather than let the firm go into disorderly fire-sale liquidation following a set of cascading cross defaults."

${ }^{6}$ Several attempted bail-ins failed because the threat of not undertaking a bail-out was not credible; see Stiglitz (2002). 


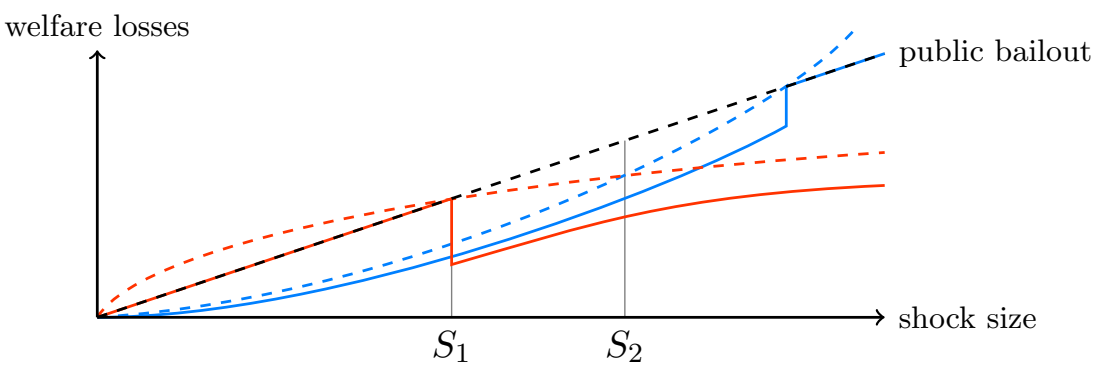

Figure 1: The figure compares equilibrium welfare losses in a diversified (blue) and a concentrated network (red) in the presence (solid lines) and absence (dashed lines) of intervention. $7^{7}$ Because the total throughput is small (large) in sparse (dense) networks, welfare losses in absence of intervention are concave (convex). When the no-intervention losses exceed the costs of a public bailout (black dashed line), the government's threat to not intervene fails to be credible and a public bailout is the only possible equilibrium rescue. If the threat is credible, contributions from the private sector are possible. Contributions are larger in the sparse network because losses without intervention are more concentrated, thereby reducing free-riding incentives. Without intervention or in a model with bailouts only, the diversified network is preferable up until shock size $S_{2}$. Endogeneity of the intervention reverses the relative desirability of the two network structures for intermediate shock sizes between $S_{1}$ and $S_{2}$.

throughput is small, the systemic threat does not increase much with the size of the shock. By contrast, in more diversified network structures, small losses can be well absorbed and the threat not to intervene is credible. However, because the total throughput is large, the threat becomes less credible as the shock size increase. As illustrated in Figure 1, endogeneity of the default resolution plan thus reverses the relative desirability of network structures for intermediate shock sizes when compared to models without intervention or models with bailouts only.

In any intervention plan - bail-in or bailout - the regulator trades off the cost of taxpayer contributions with the fire-sale losses caused by inefficient asset liquidation and broader societal losses from a bankruptcy cascade. In a bail-in, this trade-off is further affected by the size of the banks' incentive-compatible contributions, which are increasing in the attained price: the higher the asset price under the proposed resolution, the more attractive this resolution becomes relative to the default cascade where asset prices would collapse. The regulator thus sustains a higher asset price in a bail-in than in a bailout. We show that banks, in fact, benefit from additional market illiquidity. Not only does it decrease the credibility of the regulator's threat not to intervene, but the larger potential knock-on effects on the asset price also warrant larger liquidity assistance from the regulator. This results in a net increase of asset prices - at the cost of taxpayer contributions. These findings imply that banks have an incentive to specialize in thinly-traded assets. Policies which require banks to hold a certain proportion of highly liquid assets, such as the liquidity coverage ratio, thus help improve the credibility of the no-intervention threat.

We then proceed to investigate the structure of optimal intervention plans, that is, the set of banks that are rescued. A bank is not rescued in a bailout if the loss-absorption capacity of its creditors is high enough to absorb a significant portion of the losses due to contagion. In a bail-in, not only the total loss absorption capacity matters but also its distribution among the creditors. When a bank has a single creditor, that creditor cannot free-ride on the contributions of others. Proposing a bail-in for a bank whose losses are absorbed by a single creditor thus leads to an increase in welfare. However, if contagion effects are spread among many creditors, rescuing that 
bank does not substantially increase contributions from the private sector because of the banks' inherent free-riding incentives. It may then be welfare-enhancing to not rescue such a bank. Banks rescued in the optimal bail-in may thus default in the optimal bailout and vice versa.

Our results uncover the economic forces behind the decision to rescue banks which default due to idiosyncratic fundamental shocks versus those failing as a result of contagion. For example, the government opted to rescue AIG as opposed to Goldman Sachs during the global financial crisis 8 Because loss absorption capacities were low in the aftermath of Lehman Brother's default, bankruptcy filings of AIG might have had far-reaching consequences, bringing under many of its creditors including Goldman Sachs. If the financial system had been in a more resilient state, in which Goldman Sachs was the only contagiously defaulting bank, it might have been welfareenhancing to let AIG default and provide liquidity assistance only to Goldman Sachs after the depletion of Goldman's capital buffers.

The remainder of the paper is organized as follows. In Section 1, we put our work in perspective with the existing literature. We develop the model in Section 2. We characterize incentives and the equilibrium intervention outcome for any financial network in Section 3. We analyze the impact of the network structure, the shock size, and asset illiquidity on the public bailout and the credibility in Section 4. In Section 5, we characterize the set of banks to be optimally rescued in bail-ins and bailouts. We discuss the impact of the network structure on the equilibrium intervention plan in Section 6 using a model calibrated to a data set of the European Banking Authority (EBA). Section 7 concludes. The proofs of the main results are contained in the appendix. Supplementary results and auxiliary proofs are in the online appendix Bernard, Capponi and Stiglitz (2019).

\section{Literature Review}

Our paper is related to a vast branch of literature on financial contagion in interbank networks. Pioneering works include Allen and Gale (2000) and Eisenberg and Noe (2001), and further developments were made in more recent years by Acemoglu, Ozdaglar and Tahbaz-Salehi (2015), Elliott, Golub and Jackson (2014), Gai, Haldane and Kapadia (2011), Glasserman and Young (2015), and Capponi, Chen and Yao (2016). We refer to Glasserman and Young (2016) for a thorough survey on financial contagion 9 These works study how an initial shock is amplified through the interbank network based on the network structure, the inefficient liquidation of non-interbank assets, the trade-off between diversification and integration on the level of interbank exposures, and the complexity of the network. Different from our study, in these models agents execute exogenously specified contractual agreements but do not take any strategic action to resolve distress in the net-

\footnotetext{
${ }^{8}$ The bailout of AIG was widely speculated to be an indirect bailout of Goldman Sachs, to which it had sold millions of dollars worth of insurance. Because of the large exposure, AIG's default might have lead to the contagious default of Goldman Sachs, the largest investment bank at the time.

${ }^{9}$ Other related contributions include Gai and Kapadia (2010), which analyze how knock-on effects of distress can lead to write down the value of institutional assets; Cifuentes, Ferrucci and Shin (2005), which analyze the impact of fire sales on financial network contagion; Cabrales, Gottardi and Vega-Redondo (2017), which study the trade-off between the risk-sharing generated by more dense interconnection and the greater potential for default cascades; Battiston et al. (2012), which demonstrate that systemic risk does not necessarily decrease if the connectivity of the underlying financial network increases; and Elsinger, Lehar and Summer (2006), which study transmission of contagion in the Austrian banking system;
} 
work. A different branch of the literature has focused on the coordinating role of the central bank in stopping financial contagion through provision of liquidity (Freixas, Parigi and Rochet (2000) and Gorton (2010)). These studies, however, do not consider resolution strategies that involve the private sector, such as the bail-ins considered in our paper. The viability of private-sector involvement depends on the credibility of the no-intervention commitment by the government, which we show to be tightly linked to the network structure. The importance of integrating bottom up approaches based on network models and top down frameworks that design regulatory measures is stressed in a recent survey on systemic risk by Benoit et al. (2017).

In our model, the regulator determines the optimal rescue plan after the banks have already decided on the amount of risk undertaken, and shocks to outside assets have occurred. We do not consider here the moral hazard problem of banks taking excessive risks, knowing that they will be rescued if the market moves against them. Moral hazard problems arising in this context have been thoroughly investigated in the literature, though not in the context of a strategic bail-in policy. Doing so represents a next step in our research agenda, discussed briefly in Section 7. Important contributions include Gale and Vives (2002), who argue that a bailout is optimal ex post, but ex ante it should be limited to control moral hazard; Acharya and Yorulmazer (2007), who show that banks may find it optimal to invest in highly correlated assets in anticipation of a bailout triggered by the occurrence of many simultaneous failures; Farhi and Tirole (2012), who support Acharya and Yorulmazer (2007)'s findings by showing that safety nets can provide perverse incentives and induce correlated behavior that increases systemic risk; Chari and Kehoe (2016), who show that if the regulator cannot commit to avoid bailouts ex post, then banks may overborrow ex ante; and Keister (2016), who finds that prohibiting bailouts may lead intermediaries to invest into too liquid assets which lower aggregate welfare. Our model, like the rest of the literature on default resolution policies in financial networks, does not account for the endogenous structure of the interbank network. The exception is a recent study by Erol (2018), which develops an endogenous network formation model in the presence of government bailouts. Different from Erol (2018), the regulator in our model is strategic and cannot commit to a bailout or a no-intervention policy.

Related to our model is the study by Rogers and Veraart (2013), which analyze situations in which banks can stop the insolvency from spreading by stabilizing the financial system through mergers. In their paper, however, a merger need not be incentive compatible for the shareholders of an individual bank and the government does not take an active role. By contrast, our model focuses on the credibility of the regulator's actions and the free-riding problem that arises because the stability of the financial system is to the benefit of every participant. Duffie and Wang (2017) consider a bargaining model, in which bail-ins are done contractually rather than through a central planner. Unlike our paper, they crucially assume the absence of cross-network externalities. In the limit as disagreement is disallowed, Duffie and Wang (2017) show that the bargaining equilibrium in a 3-bank network is efficient. In our paper, externalities from asset liquidation and bankruptcy losses are at the heart of the analysis, driving our characterization of the endogenous ex-post default resolution plan that arises from the negotiation between regulator and financial institutions. 


\section{Model}

We consider an economy consisting of $n$ risk-neutral financial institutions $i=1, \ldots, n$, called "banks", which lasts for three periods $t=0,1,2$. At the initial period, each bank $i$ is endowed with capital that it can lend to other banks, invest into a liquid asset, called "cash", or invest into an illiquid asset, yielding a random return at time $t=1$ and a non-pledgeable return at $t=2$. Because long-term returns are non-pledgeable, liabilities have to be cleared at time $t=1$. After short-term returns are realized, banks may liquidate their risky assets to help service their debt, but because of market illiquidity, doing so will cause a downward impact on the asset's price.

Banks negotiate debt contracts with each other at $t=0$. We denote by $L^{j i}$ bank $i$ 's liabilities to bank $j$ at time $t=1$ and use $L^{i}:=\sum_{j=1}^{n} L^{j i}$ to denote bank $i$ 's total interbank liabilities. Let $p^{i}$ denote the market value of bank $i$ 's liabilities. This market value may be lower than the notional value $L^{i}$ because bank $i$ may be unable to repay its liabilities in full. If $p^{i}<L^{i}$, we say that bank $i$ defaults. All interbank liabilities have equal seniority: if bank $i$ defaults, each creditor $j$ receives $\pi^{j i} p^{i}$ from bank $i$, where

$$
\pi^{j i}= \begin{cases}L^{j i} / L^{i} & \text { if } L^{i}>0 \\ 0 & \text { otherwise }\end{cases}
$$

The relative liability matrix $\pi=\left(\pi^{i j}\right)_{i, j=1, \ldots, n}$ captures the structure of the financial network. In addition to its liabilities within the financial network, bank $i$ has financial commitments $w^{i}$ outside the financial sector due at time $t=1$, which have higher seniority than the interbank liabilities. These commitments include wages, depositors' claims, and other operating expenses ${ }^{10}$

For each bank $i$, we denote by $e^{i}$ the long-term returns of the illiquid asset held by $i$. We denote by $c^{i}$ the sum of $i$ 's cash holdings and the short-term returns realized at time $t=1$. If a bank $i$ is not able to meet its liabilities out of current income, it will liquidate a portion $\ell^{i} \in\left[0, e^{i}\right]$ of its illiquid asset. Liquidation imposes a downward impact on the price $\alpha$ of the asset given by

$$
\alpha(\ell)=\exp \left(-\gamma \sum_{i=1}^{n} \ell^{i}\right)
$$

where $\gamma \geq 0$ is the elasticity of the asset price ${ }^{11}$ A defaulting bank liquidates all of its assets to repay the maximal amount to its creditors. Because liquidation is costly, a solvent bank liquidates just enough to meet its liabilities. When prices are equal to $(p, \alpha)$, bank $i$ thus liquidates an amount

$$
\ell^{i}(p, \alpha)=\min \left(\frac{1}{\alpha}\left(L^{i}+w^{i}-c^{i}-\sum_{j=1}^{n} \pi^{i j} p^{j}\right), e^{i}\right)
$$

\footnotetext{
${ }^{10}$ Our framework can accommodate lending from outside the financial sector with equal seniority as interbank liabilities by adding a "sink node" that has only interbank assets but no interbank liabilities.

${ }^{11}$ Such a downward sloping inverse demand function has also been used in Cifuentes, Ferrucci and Shin (2005). For this choice of inverse demand function, the infinitesimal price impact is proportional to the asset's price. The elasticity parameter $\gamma$ typically depends on the asset class: Ellul, Jotikasthira and Lundblad (2011) find that $\gamma$ is in the order of $10^{-8}$ for corporate bonds (see Table 8 therein).
} 
where $(\cdot)^{+}=\max (\cdot, 0)$ denotes the positive part ${ }^{12}$ If a bank $i$ cannot meet its liabilities even after liquidating all of its assets, it will default. The default creates losses proportional to the banks' asset value: only a fraction $\beta \in(0,1]$ of the bank's asset value is paid to the creditors and a fraction $(1-\beta)$ is lost ${ }^{13}$ The market value of bank $i$ 's interbank liabilities is thus equal to

$$
p^{i}= \begin{cases}L^{i} & \text { if } c^{i}+\alpha \ell^{i}+(\pi p)^{i} \geq L^{i}+w^{i} \\ \left(\beta\left(c^{i}+\alpha e^{i}+(\pi p)^{i}\right)-w^{i}\right)^{+} & \text {otherwise }\end{cases}
$$

where $(\pi p)^{i}=\sum_{j=1}^{n} \pi^{i j} p^{j}$ is the market value of bank $i$ 's interbank claims. How much a bank is able to repay depends on the solvency of the other banks in the system. In a clearing equilibrium, every solvent bank repays its liabilities in full and every insolvent bank pays its entire value (after bankruptcy costs) to its creditors.

The financial system in our model is parametrized by $(L, \pi, e, c, w, \gamma, \beta)$, where $L, e, c$, and $w$ are vectors whose entries are the corresponding balance sheet quantities of each bank.

Definition 2.1. A set of prices and liquidation decisions $(p, \ell, \alpha)$ is a clearing equilibrium for a financial system $(L, \pi, e, c, w, \gamma, \beta)$ if it satisfies (1)-(3).

When prices are equal to $(p, \alpha)$, the value of bank $i$ 's equity is given by

$$
V^{i}(p, \alpha):=\left((\pi p)^{i}+c^{i}+\alpha e^{i}-w^{i}-L^{i}\right)^{+}
$$

If the payment $p^{i}$ made by bank $i$ is positive, it is divided pro-rata among bank $i$ 's junior creditors and the senior creditors are paid in full. If $p^{i}=0$, the junior creditors lose the full amount of their claims and the senior creditors of bank $i$ suffer a loss of

$$
\delta^{i}(p, \alpha):=\left(w^{i}-\beta\left(c^{i}+\alpha e^{i}+(\pi p)^{i}\right)\right)^{+},
$$

We denote by $\mathcal{D}(p, \ell, \alpha):=\left\{i \mid L^{i}+w^{i}>c^{i}+\alpha \ell^{i}+(\pi p)^{i}\right\}$ the set of defaulting banks. Welfare losses in a clearing equilibrium are defined as the weighted sum of default costs, i.e.,

$$
W_{\lambda}(p, \ell, \alpha):=(1-\alpha) \sum_{i=1}^{n} e^{i}+(1-\beta) \sum_{i \in \mathcal{D}(p, \ell, \alpha)}\left(c^{i}+\alpha e^{i}+(\pi p)^{i}\right)+\lambda \sum_{i \in \mathcal{D}(p, \ell, \alpha)} \delta^{i}(p, \alpha) .
$$

The first term are losses due to fire sales, and the second term quantifies deadweight losses from bankruptcy. Losses of senior creditors are weighted by a factor $\lambda \geq 0$, which captures the importance the regulator assigns to the senior creditor's losses relative to deadweight losses. A regulator with $\lambda=0$ views losses of senior creditors simply as transfers of wealth and not as losses to the economy. A higher value of $\lambda$ indicates a higher priority to the economy outside of the banking sector.

\footnotetext{
${ }^{12}$ The model can be adjusted to account for liquidity requirements such as Basel-III (2013). This can be achieved by setting $c^{i}$ equal to the amount of liquid assets that can be liquidated before hitting the liquidity coverage ratio requirement.

${ }^{13}$ According to Moody's analysis, the average recovery rate for unsecured corporate bonds ranges from $30 \%$ to $43 \%$; see Exhibit 8 in https://www.moodys.com/sites/products/DefaultResearch/2006600000428092.pdf
} 
Every financial system admits a clearing equilibrium as a consequence of Tarski's fixed-point theorem. Cifuentes, Ferrucci and Shin (2005) show that it is unique under mild regularity conditions if $\beta=1$. This is no longer the case in the presence of bankruptcy costs, i.e., when $\beta<1$. Following standard practices in the literature, liabilities are then cleared with the unique Pareto-dominant clearing equilibrium.

Lemma 2.1. For any financial system, there exist a greatest and a lowest clearing equilibrium $(\bar{p}, \bar{\ell}, \bar{\alpha})$ and $(\underline{p}, \underline{\ell}, \underline{\alpha})$, respectively, with $\bar{p}^{i} \geq p^{i} \geq \underline{p}^{i}, \bar{\ell}^{i} \leq \ell^{i} \leq \underline{\ell}^{i}$, and $\bar{\alpha}^{i} \geq \alpha^{i} \geq \underline{\alpha}^{i}$ for any clearing equilibrium $(p, \ell, \alpha)$ and any bank $i$. Moreover, $(\bar{p}, \bar{\ell}, \bar{\alpha})$ is Pareto dominant for any $\lambda \geq 0$, that is, for any clearing equilibrium $(p, \ell, \alpha)$, we have $V^{i}(\bar{p}, \bar{\alpha}) \geq V^{i}(p, \alpha)$ for all $i$ and $W_{\lambda}(\bar{p}, \bar{\ell}, \bar{\alpha}) \leq W_{\lambda}(p, \ell, \alpha)$.

\subsection{Contagion and Default Cascade}

We position ourselves at time $t=1$, when short-term returns have been realized but banks have not yet cleared their liabilities. Our objective is to characterize the endogenous intervention outcome after any realization of market returns. We consider voluntary bail-ins, so that if the banks and/or the regulator have implemented automatic bail-in triggers such as, for example, contingent convertible bonds, then $(L, \pi, e, c, w, \alpha, \beta)$ represents the state of the financial system after accounting for these risk-mitigating actions. Depending on the size of the shock, banks may still need to dispose of their illiquid assets to remain solvent and defaults may still occur.

There are two channels of financial contagion in our model. The first channel is price-mediated contagion. When a distressed bank needs to liquidate the asset, the price declines due to market illiquidity, captured in reduced from through (1). As a consequence, other banks which hold the asset experience a reduction of their equity value. If the price impact from asset sales is sufficiently large, other banks may also need to liquidate some of their asset to stay solvent, thereby further depressing the price. This leads to a downward spiral, which converges to the highest price, for which the asset's demand - given by the inverse of (1) - equals the amount liquidated.

Lemma 2.2. For any vector $p$ of repayments, there exists a solution $\left(\alpha_{p}, \ell_{p}\right)$ to (1) and (2) such that $\alpha \leq \alpha_{p}$ for any other solution $(\alpha, \ell)$ to (1) and (2).

The second channel of contagion in our model is credit contagion. A defaulting bank $i$ reduces the payments to its creditors, thereby imposing losses $L^{i}-p^{i}$ to the rest of the financial system. Creditors with large interbank exposures may thus default as a consequences of these losses. This, in turn, may affect the solvency of their creditors and so on, causing a cascade of defaults. The cascade starts with the set $\mathcal{F}:=\left\{i \mid c^{i}+\alpha_{L} \ell_{L}^{i}+(\pi L)^{i}<L^{i}+w^{i}\right\}$ of fundamentally defaulting banks, i.e., the set of banks that are unable to repay their liabilities even if every other bank repays its liabilities in full. If there are no fundamentally defaulting banks, then $\left(L, \ell_{L}, \alpha_{L}\right)$ is the Pareto-efficient clearing equilibrium and all interbank liabilities are honored. 


\subsection{Coordination of Rescues}

As argued in the preceding section, financial stability can be ensured by injecting sufficient liquidity into the fundamentally defaulting banks so that they can meet their liabilities. In a bailout, this additional liquidity is provided by the government through taxpayer contributions, whereas in a bail-in, creditors of insolvent banks voluntarily take a haircut on their claims. As an intermediate option between the two, we also consider assisted bail-ins, which include transfers by both the banks and the government.

Definition 2.2. An assisted bail-in $(b, s)$ specifies, for each bank $i$, the contribution $b^{i}$ to be made by $i$ and the size $s^{i}$ of the subsidy $i$ receives. The government's contribution to the bail-in is $\sum_{i=1}^{n}\left(s^{i}-b^{i}\right)$, which is imposed to be non-negative 14

Observe that assisted bail-ins contain bailouts and privately backed bail-ins as special cases. A bailout is the limiting case of an assisted bail-in, in which the banks' contributions are equal to zero. In a private bail-in, the government contributions are zero. In addition to contributing to a bail-in financially, the regulator also serves to coordinate among different bail-ins. Specifically, the regulator may propose a bail-in allocation, but cannot force banks to participate.

\section{Organization of a rescue.}

1. The regulator proposes an assisted bail-in $(b, s)$.

2. Each bank $i$ from the set $\mathcal{A}(b):=\left\{j \notin \mathcal{F} \mid b^{j}>0\right\}$ chooses a binary action $a^{i} \in\{0,1\}$, indicating whether or not it agrees to contribute $b^{i} 15$

3. The regulator chooses his response $r$ from the following three options:

(i) "bail-in": Proceed with the proposed subsidies $s$, using taxpayer money to cover the contributions of banks which rejected the proposal. Cash holdings and financial commitments of each bank $i$ are then equal to $c^{i}(s):=c^{i}+s^{i}$ and $w^{i}(b, a):=w^{i}+b^{i} 1_{\left\{a^{i}=1\right\}}$ and the resulting financial system is cleared as in Section 2. We denote by $\bar{p}(b, s, a)$ and $\bar{\alpha}(b, s, a)$ the prices in the Pareto-dominant clearing equilibrium. Bank $i$ 's equity value is equal to $V^{i}(\bar{p}(b, s, a), \bar{\alpha}(b, s, a))$. Welfare losses are obtained from (6) by additionally accounting for the social cost of government subsidies, that is,

$$
W_{\lambda}(b, s, a):=W_{\lambda}(\bar{p}(b, s, a), \bar{\ell}(b, s, a), \bar{\alpha}(b, s, a))+\lambda \sum_{i=1}^{n}\left(s^{i}-b^{i} 1_{\left\{a^{i}=1\right\}}\right) 16
$$

\footnotetext{
${ }^{14}$ This formulation is equivalent to a formulation, in which creditors write down their claims on insolvent banks such that $b^{i}$ is the net debt forgiven by bank $i$ and $s^{i}$ is the sum of government injections and net debt forgiven to bank $i$ by $i$ 's creditors.

${ }^{15} \mathrm{We}$ assume that a bank $i$ with $b^{i}=0$ is simply not part of the negotiation and hence has no power to reject the proposal. For ease of notation, we write $a^{i}=1$ for such a bank.

${ }^{16} \mathrm{We}$ assume that the regulator assigns the same weight to taxpayer contributions as to losses of senior creditors. This assumption is made for notational convenience and our results would hold if the regulator used different weights.
} 
(ii) "bailout": Resort to a public bailout $(0, \tilde{s})$ with subsidies $\tilde{s}$ decided by the regulator. Then, cash holdings of bank $i$ are equal to $c^{i}(\tilde{s})=c^{i}+\tilde{s}^{i}$ and we denote by $\bar{p}(\tilde{s})$ and $\bar{\alpha}(\tilde{s})$ the prices in the Pareto-dominant clearing equilibrium. Each bank $i$ 's equity value is $V^{i}(\bar{p}(\tilde{s}), \bar{\alpha}(\tilde{s}))$ and welfare losses are denoted by $W_{\lambda}(\tilde{s}):=W_{\lambda}(0, \tilde{s}, 0)$.

(iii) "no intervention": Abandon the rescue, which results in the default cascade of Section 2 . We denote by $\left(p_{N}, \ell_{N}, \alpha_{N}\right)$ the Pareto-dominant clearing equilibrium and by $W_{N}$ the welfare losses in the default cascade.

Remark 2.1. Some banks may be left with zero equity after they are bailed in or bailed out. Such banks cease to exist as a separate entity after the intervention and their bail-ins or bailouts should be understood as an orderly liquidation through takeovers by the bail-in contributors or the government 17 Because these banks have zero equity value after the intervention, we do not model how the assets are distributed among contributors.

A strategy of each bank $i$ is a map $a^{i}(b, s)$ that specifies for each proposal whether or not $i$ agrees to participate. The regulator's strategy consists of which bail-in $(b, s)$ to propose, as well as a reaction $r(b, s, a)$ to any given response vector $a$ by banks. Our solution concept is that of subgame Pareto efficient equilibria, defined as follows.

Definition 2.3. A strategy profile $(b, s, a, r)$ is subgame Pareto efficient if it is subgame perfect and after any proposal $(b, s)$, there is no other continuation equilibrium $(\tilde{a}, \tilde{r})$ of the accepting/rejecting subgame that Pareto dominates $(a, r)$ for the banks in $\mathcal{A}(b)$ and the regulator.

This equilibrium solution concept is meant to capture the interactions between the regulator and the banks in $\mathcal{A}(b)$, aiming at finding a suitable resolution outcome. For example, during the bailin of Long-Term Capital Management, Peter Fisher of the Federal Reserve Bank of New York sat down with representatives of LTCM's creditors to find an appropriate solution; and it is implausible that they would have agreed on a bail-in plan that is Pareto dominated. Note that banks in the complement of $\mathcal{A}(b)$ are potentially worse off than in an alternative continuation equilibrium of the accepting/rejecting subgame because they are not part of the discussion.

Any proposal of the regulator admits an equilibrium response by the banks by the following lemma. This result also implies existence of subgame Pareto efficient equilibria in our model.

Lemma 2.3. After any proposal $(b, s)$, there exists a Pareto efficient continuation equilibrium $(a, r)$ of the accepting/rejecting subgame.

Because subgame Pareto efficiency is a refinement of subgame perfection, the regulator lacks commitment power: it eliminates the non-credible threat of the regulator to abandon the rescue in the third stage when, in fact, a public bailout leads to lower welfare losses than a default cascade. This restricts the regulator's ability to incentivize banks to contribute as we discuss in Section 3.2 .

\footnotetext{
${ }^{17}$ Examples of such takeovers from the global 2007-2009 financial crisis are plentiful. Among the most prominent ones are the takeovers of Bear Stearns and Merrill Lynch by JP Morgan Chase and Bank of America, respectively, or the federal takeovers of Fannie Mae and Freddie Mac.
} 
In our model, the regulator knows the financial position of each bank and, thus, he can anticipate the banks' responses to any bail-in proposal. Therefore, he need not make a proposal that is not incentive compatible and the negotiation is collapsed into a single stage. In reality, the regulator typically is not fully informed and the coordination of a bail-in might take the form of a strategic bargaining game instead. Some banks might reject the regulator's initial proposal, after which the regulator revises his proposal to either exclude those banks or to accommodate them 18

We may assume without loss of generality that the regulator proposes only so-called feasible bail-ins, in which every bank can afford the proposed contribution.

Definition 2.4. A bail-in proposal $(b, s)$ is feasible if $b^{i}=0$ for any fundamentally defaulting bank $i \in \mathcal{F}$ and $L^{i}+w^{i}+b^{i} \leq c^{i}+s^{i}+\bar{\alpha}(b, s, 1) \ell^{i}(b, s, 1)+\sum_{j=1}^{n} \pi^{i j} \bar{p}^{j}(b, s, 1)$ for any bank $i \notin \mathcal{F}$, where $1=(1, \ldots, 1)$ is the response of unanimous agreement.

Since prices are weakly decreasing with the banks' contributions, feasibility of a bail-in $(b, s)$ implies that $L^{i}+w^{i}+b^{i} \leq c^{i}+s^{i}+\bar{\alpha}(b, s, a) \ell^{i}(b, s, a)+\sum_{j=1}^{n} \pi^{i j} \bar{p}^{j}(b, s, a)$ also for any response vector $a \neq(1, \ldots, 1)$. Therefore, whether a bank can afford to contribute to a feasible bail-in does not depend on the responses of the other banks. Key is the fact that if some bank $i$ refuses to participate but the regulator decides to implement the bail-in nonetheless, then the regulator uses additional taxpayer money to make up for $i$ 's contribution. Feasibility of a proposed bail-in thus provides banks with a guarantee that prices will be at least $\bar{p}(b, s, 1)$ and $\bar{\alpha}(b, s, 1)$-backed by government contributions if necessary; see Lemma F.3 in the online appendix for details 19

\section{Incentives and Credibility of Intervention Plans}

To highlight the primary economic forces at play, we focus on the case of what we call complete interventions in this section, where the regulator considers only bailouts and bail-ins that rescue every bank in the system. Section 5 treats the more general case where the regulator can propose interventions that rescue some banks but not others, and discusses the additional economic tradeoffs arising in the equilibrium outcome.

\subsection{Public Bailout}

In a complete bailout, the regulator provides subsidies to support the clearing payment vector $L$. The smallest subsidies necessary are those required to make every bank solvent, which are equal to

$$
s_{L}:=\left(L+w-c-\alpha_{L} \ell_{L}-\pi L\right)^{+},
$$

\footnotetext{
${ }^{18}$ The original proposal made by the Federal Reserve Bank of New York for the rescue of Long Term Capital Management involved a total of 16 of LTCM's creditors. However, Bear Stearns and Lehman Brothers later declined to participate. Upon the rejection of these two banks, the Fed adjusted its proposal so that the contributions of Bear Stearns and Lehman Brothers were covered by the remaining 14 banks. The fact that these two banks decided not to cooperate shows that participation in the bail-in was at least partially voluntary.

${ }^{19}$ In reality, asset price guarantees are often given through collateral. This was the case in the purchase of Bear Stearns by JP Morgan Chase, where the government provided a large collateral for Bear Stearns' assets.
} 
where $\left(\alpha_{L}, \ell_{L}\right)$ is given by Lemma 2.2 . If asset liquidation is more costly than tax- payer contributions, the regulator will want to provide additional subsidies up to

$$
s_{0}:=(L+w-c-\pi L)^{+}
$$

so that no bank liquidates any of its assets. Note that subsidies $s_{L}$ and $s_{0}$ support the clearing equilibria $\left(L, \ell_{L}, \alpha_{L}\right)$ and $(L, 0,1)$, respectively. The welfare-maximizing subsidies $s$ in a complete bailout are such that the marginal losses from liquidation are as close to the marginal welfare cost $\lambda$ of taxpayer contributions as possible, given the constraints $s_{L}^{i} \leq s^{i} \leq s_{0}^{i}$, which guarantee solvency of every bank in the system.

Lemma 3.1. Define the function $g(\alpha)=\alpha\left(\frac{\lambda}{\gamma} \ln (\alpha)-\sum_{i=1}^{n} e^{i}\right)$. In any complete bailout with subsidies $s^{i} \leq s_{0}^{i}$ for every bank $i$, welfare losses are equal to

$$
W_{\lambda}(s)=\sum_{i=1}^{n}\left(e^{i}+\lambda s_{0}^{i}\right)+g(\bar{\alpha}(s)) .
$$

The regulator's choice of subsidies affects welfare only through the induced asset price as the argument of the function $g$, which captures the welfare trade-off between liquidation costs and taxpayer contributions. Any bailout that induces asset price $\alpha$ requires banks to liquidate an aggregate amount $-\ln (\alpha) / \gamma$ as seen in (1). This corresponds to a reduction of the maximal subsidies $s_{0}$ by the market value of those liquidated assets. Choosing subsidies that induce asset price $\alpha$ (as opposed to $s_{0}$ ) thus lower welfare costs by $-\lambda \alpha \ln (\alpha) / \gamma$ but increase liquidation losses by $\alpha \sum_{i=1}^{n} e^{i}$. The function $g$ is convex and it is minimized at the indifference price

$$
\alpha_{\text {ind }}:=\exp \left(\frac{\gamma}{\lambda} \sum_{i=1}^{n} e^{i}-1\right)
$$

If $\alpha<\min \left(\alpha_{\text {ind }}, 1\right)$, liquidation is more costly than taxpayer contributions, hence the regulator will provide additional subsidies to drive the price up to $\min \left(\alpha_{\text {ind }}, 1\right)$. If $\alpha>\alpha_{\text {ind }}$, asset liquidation is considered cheaper than taxpayer contributions, hence the regulator will reduce the size of the provided subsidies (to a minimum of $s_{L}$ ) to drive the price down to $\min \left(\alpha_{\text {ind }}, \alpha_{L}\right)$.

Lemma 3.2. Let $\alpha_{P}=\max \left(\min \left(\alpha_{\mathrm{ind}}, 1\right), \alpha_{L}\right)$. A welfare-maximizing complete bailout awards subsidies $s$ with $s_{L}^{i} \leq s^{i} \leq s_{0}^{i}$ for every bank $i$ such that

$$
\sum_{i=1}^{n} s^{i}=\sum_{i=1}^{n} s_{0}^{i}+\frac{\alpha_{P} \ln \left(\alpha_{P}\right)}{\gamma} .
$$

These subsidies induce asset price $\alpha_{P}$. We denote by $W_{P}$ the corresponding welfare losses.

If $\alpha_{P}=\alpha_{L}$ or $\alpha_{P}=1$, subsidies in the bailout are unique and equal to $s_{L}$ and $s_{0}$, respectively. However, subsidies are not unique if $\alpha_{L}<\alpha_{P}<1$ : since the price impact depends only on the total 
amount of liquidation, welfare depends only on the total subsidies awarded, but not on how those are distributed among banks. The regulator wishes to prevent any asset liquidation if $\alpha_{P}=1$. It follows from (10) that this is the case if and only if $\lambda \leq \gamma \sum_{i=1}^{n} e^{i}$, i.e., if taxpayer contributions are considered cheap, if the asset price is sufficiently elastic, or if the total holdings in the illiquid asset exceed the threshold $\lambda / \gamma$. Depending on the distribution of the shortfall, the regulator may not be able to attain the indifference price in a complete bailout. Because the minimal required subsidies $s_{L}$ induce asset price $\alpha_{L}$, it is impossible to do so if $\alpha_{\text {ind }}<\alpha_{L}$. Note that the optimal complete bailout does not depend on the network structure because all defaults are prevented.

\subsection{Credibility of the Regulator's Threat}

If welfare losses in the public bailout of Lemma 3.2 are lower than in a default cascade, banks know that the regulator's preferred outside option is a bailout. The regulator thus has no credible threats to punish recalcitrant banks and, as a consequence, banks have no incentive to participate in any bail-in the regulator proposes. The following proposition shows that in such a situation, the regulator has no choice but to resort to a public bailout.

Proposition 3.3. If $W_{P}<W_{N}$, the unique subgame Pareto efficient equilibrium outcome is the public bailout of Lemma 3.2.

We say that the regulator's no-intervention threat is credible if and only if $W_{N} \leq W_{P}$. We will show in the the following three subsections that when the threat is credible, the regulator can indeed incentivize banks to participate in a bail-in. In the remainder of this section, we study the conditions under which the threat is credible 20

The main component of welfare losses in a bailout is the banks' aggregate shortfall $\sum_{i=1}^{n} s_{0}^{i}$, where $s_{0}$ is defined in (8). The aggregate shortfall is a measure of the size of the initial shock hitting the financial system, when the shock is large enough that banks can no longer pay their liabilities out of current income. Welfare losses in a default cascade are a measure of the size of the shock after it propagates through the financial system. Losses of junior creditors in a default cascaded are

$$
S_{N}:=\sum_{i=1}^{n}\left(V^{i}(L, 1)-V^{i}\left(p_{N}, \alpha_{N}\right)\right)
$$

The following result states that the regulator's threat is credible if and only if the amplification of losses through the financial system in absence of intervention is smaller than a certain threshold. Because senior creditors may absorb a portion of the losses, the part of the initial shock that is amplified through the network is

$$
S_{0}:=\sum_{i=1}^{n} s_{0}^{i}-\sum_{i \in \mathcal{D}\left(p_{N}, \ell_{N}, \alpha_{N}\right)} \delta^{i}\left(p_{N}, \alpha_{N}\right) .
$$

\footnotetext{
${ }^{20}$ Note that the credibility of the threat is a function of exogenous variables: the welfare losses $W_{P}$ in the optimal bailout is the result of a minimization problem solved by the regulator alone and $W_{N}$ are the welfare losses in absence of any action.
} 
Lemma 3.4. The regulator's threat is credible if and only if

$$
S_{N}-S_{0} \leq \lambda S_{0}+\sum_{i=1}^{n}\left(e^{i}-s_{0}^{i}\right)^{+}+g\left(\alpha_{P}\right) .
$$

The credibility threshold depends on the weight $\lambda$ given to taxpayer contributions in the social welfare function, the size of the initial shortfall, and the distribution of the initial shortfall via the second and third term on the right-hand side of $(12)$. The second term is a measure of the amount of illiquid assets that can be used to absorb the initial shock: if a larger part can be absorbed by the system, the threat is more credible. The last term in $(12)$ captures the trade-off between liquidation costs and taxpayer contributions in the optimal bailout for a given distribution of shocks $s_{0}$. While this trade-off is minimized at the indifference price $\alpha_{\text {ind }}$, the regulator may not be able to attain $\alpha_{\text {ind }}$ for any distribution of shocks $s_{0}$ as seen in Lemma 3.2. The difference $g\left(\alpha_{P}\right)-g\left(\alpha_{\text {ind }}\right)$ is thus a measure for how close to attaining $\alpha_{\text {ind }}$ the regulator can tailor a bailout for a given distribution of shocks; see also the discussion after Lemma 3.1 .

Lemma 3.4 establishes a link between the credibility of the no-intervention threat and the existing literature on financial networks without intervention, which often ranks the desirability of network structures according to the welfare loss criterion $S_{N}-S_{0}$. Existing results in Allen and Gale (2000) and Acemoglu, Ozdaglar and Tahbaz-Salehi (2015) show that dense connections between banks may serve as an amplifier for large initial shocks. Since the right-hand side of (12) does not depend on the network structure, Lemma 3.4 indicates that dense connections are detrimental to the credibility of the threat when the initial shock is large.

\subsection{Regulator's Response in the Last Stage}

It follows as a consequence to Lemma 3.2 that, for a given bail-in proposal $(b, s)$ and the banks' response vector $a$, the regulator's best response in stage 3 is

$$
r(b, s, a)= \begin{cases}\text { "no intervention" } & \text { if } W_{N} \leq \min \left(W_{\lambda}(b, s, a), W_{P}\right), \\ \text { "bailout" } & \text { if } W_{P}<\min \left(W_{N}, W_{\lambda}(b, s, a)\right), \\ \text { "bail-in" } & \text { otherwise. }\end{cases}
$$

The regulator chooses the action that minimizes welfare losses if such an action is unique. Ties are broken according to "no intervention" $\succ$ "bail-in" $\succ$ "bailout" so that (a) taxpayer money is used only if it is strictly welfare increasing and (b) unilateral deviations by banks in stage 2 can be discouraged when $W_{N}=W_{\lambda}(b, s, a)$; see Lemma 3.5 and Footnote 21 below for details.

\subsection{Banks' Equilibrium Responses}

In this section, we analyze how the banks respond to a given proposal when the regulator's threat is credible. A crucial separation among the banks' responses is whether or not they trigger the regulator's decision to proceed with the bail-in. 
Definition 3.1. Given a bail-in proposal $(b, s)$, an equilibrium response $a$ is an accepting equilibrium if $r(b, s, a)=$ "bail-in" and it is a rejecting equilibrium otherwise.

Remark 3.1. The banks' responses do not have to be unanimous in an accepting or rejecting equilibrium. Some banks may reject the proposal in an accepting equilibrium and vice versa. However, a sufficient proportion of banks accepts the proposal in an accepting equilibrium for the regulator to proceed with the bail-in.

For a bank to participate in a bail-in, the bail-in has to be incentive-compatible. Proposition 3.3 shows that when the regulator's threat fails to be credible, the regulator can do no better than resorting to the public bailout of Lemma 3.2 . If the threat is credible, the incentive-compatibility conditions are stated in the following lemma.

Lemma 3.5. Suppose that $W_{N} \leq W_{P}$. Let $(b, s)$ be a feasible proposal of a complete bail-in. In any accepting equilibrium $a$, bank $i$ with $b^{i}>0$ accepts if and only if:

1. $W_{\lambda}\left(b, s,\left(0, a^{-i}\right)\right) \geq W_{N}$, and

2. $b^{i}-s^{i} \leq \sum_{j=1}^{n} \pi^{i j}\left(L^{j}-p_{N}^{j}\right)+\left(\alpha\left(b, s,\left(1, a^{-i}\right)\right)-\alpha_{N}\right) e^{i}$.

The first condition states that there is no possibility for free-riding: if bank $i$ were to reject the proposal, the regulator would not make up for $i$ 's contribution and he would let a default cascade occur instead ${ }^{21}$ In other words, the set of banks which accept the proposal is minimal in any accepting equilibrium. The second condition states how much bank $i$ is willing to contribute to prevent a default cascade. Bank $i$ is willing to make a net contribution up to the sum of its exposure to the default cascade through both channels of contagion.

Accepting equilibria need not be unique: for a given proposal, the regulator may be happy to organize a bail-in with more than one accepting coalition of banks. This raises the questions of how banks coordinate among different equilibrium responses. The following result shows that the regulator can preempt any problems of coordination by altering the proposed bail-in so that it is incentive compatible only for one consortium of banks to accept the proposal. If the regulator requests 0 contributions from any bank outside the selected consortium, unanimous acceptance becomes the unique accepting equilibrium of the revised proposal.

Lemma 3.6. Let $(b, s)$ be a bail-in with accepting equilibria $\left\{a_{1}, \ldots, a_{m}\right\}$. For any $k=1, \ldots, m$, there exists a proposal $(\tilde{b}, \tilde{s})$ with $W_{\lambda}\left(b, s, a_{k}\right)=W_{\lambda}(\tilde{b}, \tilde{s}, 1)$, to which $1=(1, \ldots, 1)$ is the unique accepting equilibrium response.

Our next result shows the banks will not reject a proposal if there is a subgame Pareto efficient equilibrium outcome in which the bail-in is implemented.

Lemma 3.7. Suppose that a proposal $(b, s)$ admits at least one accepting equilibrium. Then the accepting equilibrium leading to the lowest welfare losses is subgame Pareto efficient. Moreover, a rejecting equilibrium is subgame Pareto efficient if and only if $(b, s)$ is the bailout given by Lemma 3.2.

\footnotetext{
${ }^{21}$ The regulator prefers "no intervention" over "bail-in" in 13 when they lead to the same welfare losses so that a rejection by bank $i$ can be prevented when $W_{\lambda}\left(b, s,\left(0, a^{-i}\right)\right)=W_{N}$.
} 
If a proposal admits a unique accepting equilibrium, its acceptance is the unique subgame Pareto efficient continuation by Lemma 3.7. For such a proposal, the regulator can anticipate the banks' responses and what the resulting welfare losses will be. It is thus suboptimal for the regulator to make a proposal that admits more than one accepting equilibrium: according to Lemma 3.6, the regulator could have revised his proposal to select his preferred response unambiguously. Among those proposals that admit only one accepting equilibrium, the regulator will thus propose the bailin that minimizes welfare losses, subject to the incentive-compatibility conditions of Lemma 3.5 . This is described in the following section.

\subsection{Optimal Proposal of the Regulator}

Contributions of banks to a bail-in affect welfare in two ways. First, they reduce the amount of taxpayer contributions needed. Second, if the asset price in the optimal bailout is larger than the indifference price, the regulator can use the contributions of banks to enhance welfare by exploiting the trade-off between asset liquidation and taxpayer contributions.

Lemma 3.8. Let $g$ and $s_{0}$ be defined as in Lemma 3.1 and (8), respectively, and let $b_{0}:=(c+$ $\pi L-w-L)^{+}$. Let $(b, s)$ be a complete feasible bail-in proposal with $b^{i} s^{i}=0$ for every bank $i .22$ For any response vector a, welfare losses are equal to

$$
W_{\lambda}(b, s, a)=W_{P}+\left(g(\bar{\alpha}(b, s, a))-g\left(\alpha_{P}\right)\right)+\lambda \sum_{i=1}^{n}\left(s^{i}-s_{0}^{i}\right)^{+}-\lambda \sum_{i=1}^{n} \min \left(b^{i}, b_{0}^{i}\right) 1_{\left\{a^{i}=1\right\}} .
$$

Equation (14) shows how welfare losses in a bail-in compare to welfare losses in the optimal bailout of Lemma 3.2. A contribution of bank $i$ up to the amount $b_{0}^{i}$ does not require asset liquidation, hence it does not impact the asset price. Thus, each dollar contributed up to $b_{0}^{i}$ improves welfare by $\lambda$. Contributions in excess of $b_{0}^{i}$ require asset liquidation by the bank, thereby impacting the asset price and the welfare trade-off $g$. Finally, subsidies beyond $s_{0}$ do not bring any benefit in terms of reduced fire-sale losses because banks can fulfill all obligations without liquidating assets. Each dollar of subsidies awarded in excess of $s_{0}$ thus effectively burns $\lambda$ units of welfare. While this generally constitutes a decrease in welfare, we illustrate below how welfare burning can be used by the regulator to deter banks from free-riding.

Next, we analyze how the regulator best implements a rescue plan that satisfies the incentivecompatibility conditions of Lemma 3.5. The no-free-riding constraint in Condition 1 requires that after the rejection by any bank, welfare losses in the residual bail-in are larger than welfare losses in a default cascade. Using (14), this is equivalent to requiring that for any participating bank $i$,

$$
W_{\lambda}(b, s, a) \geq W_{N}+g(\bar{\alpha}(b, s, a))-g\left(\bar{\alpha}\left(b, s,\left(0, a^{-i}\right)\right)\right)-\lambda \min \left(b^{i}, b_{0}^{i}\right)
$$

Equation (15) constitutes a lower bound on attainable welfare losses imposed by the no free-riding

\footnotetext{
${ }^{22}$ The restriction $b^{i} s^{i}=0$ imposes that bank $i$ either receives subsidies or makes contributions to the bail-in, but not both. We show in Lemma C.1 in the appendix that this comes without loss of generality.
} 
constraint. It states that welfare losses in an incentive-compatible bail-in cannot be lowered from $W_{N}$ by more than the welfare impact stemming from the contribution of any participating bank.

The no-free-riding constraint thus drives the regulator to include in the bail-in banks which have a potential for large contributions or banks whose contributions enhance the welfare trade-off between subsidies and asset liquidation. It follows from Condition 2 in Lemma 3.5 that for a given set of liquidation decisions $\ell$ that induces asset price $\alpha$, the vector of largest incentive-compatible contributions is

$$
\eta(\alpha, \ell):=\min \left(\pi\left(L-p_{N}\right)+\left(\alpha-\alpha_{N}\right) e,(c+\alpha \ell+\pi L-w-L)^{+}\right) .
$$

The right expression in the minimum appearing in $(16)$ is the vector of budget constraints in a bail-in, in which the banks' contributions induce the amount $\ell$ of asset liquidation ${ }^{23}$ Note that by choosing an incentive-compatible proposal $(b, s)$, the regulator implicitly chooses an associated vector of liquidation decisions $\ell(b, s, 1){ }^{24}$ In order to construct a bail-in with maximal contributions by the banks, the regulator can thus choose $\ell$ first and propose a bail-in with contributions $\eta(\alpha(\ell), \ell)$. For a chosen set of liquidation decisions $\ell$ that induce asset price $\alpha$, the necessary subsidies to guarantee solvency of every bank are

$$
s(\alpha, \ell):=(L+w-c-\alpha \ell-\pi L)^{+}
$$

We say that a bail-in is individually incentive compatible if Condition 2 of Lemma 3.5 is satisfied for each bank when every bank accepts the regulator's proposal. The following lemma states that bailins of the above form are welfare-maximizing among individually incentive-compatible bail-ins. 25

Lemma 3.9. Let $(b, s)$ be a complete feasible bail-in that satisfies Condition 2 of Lemma 3.5 for every bank in the response vector $1=(1, \ldots, 1)$. Let $\mathcal{C}:=\left\{i \mid b^{i}>0\right\}$ and denote $\alpha=\bar{\alpha}(b, s, 1)$. Then

$$
W_{\lambda}(b, s, 1) \geq W_{P}-g\left(\alpha_{P}\right)+g(\alpha)-\lambda \sum_{i \in \mathcal{C}} \eta^{i}(\alpha, 0) .
$$

Equality holds in (18) if and only if $b^{i}-s^{i} \geq \eta^{i}(\alpha, 0)$ for every $i \in \mathcal{C}$ and $s^{i} \leq s_{0}^{i}$ for every $i \notin \mathcal{C}$.

Equation (18) shows that when contributions are of size $\eta(\alpha, \ell)$, their welfare impact depends on the liquidation decision only through the induced asset price. Thus, similarly to the bailout, the regulator optimizes bail-ins to induce the asset price, at which he is indifferent between additional taxpayer contributions and asset liquidation. Since banks are willing to make larger contributions to a bail-in that guarantees a higher asset price by Lemma 3.5, the regulator's indifference price in a bail-in is higher than in a bailout.

\footnotetext{
${ }^{23}$ The model could easily be adapted to allow for capital requirements of solvent banks by subtracting the capital requirements from the budget constraint in the right expression of 16 .

${ }^{24}$ By Lemma 3.6 the regulator can aim to construct bail-ins that can be accepted by all banks without loss of generality.

${ }^{25}$ Feasibility and individual incentive compatibility imply that $b^{i}-s^{i} \leq \eta^{i}(\alpha, e)$ for any contributing bank $i$. Since every bank is rescued in a complete bail-in, subsidies satisfy $s^{i} \geq s^{i}(\alpha, e)$ for every $i \notin \mathcal{C}$. The lower bound in (18) is thus attained if and only if $\eta^{i}(\alpha, 0) \leq b^{i}-s^{i} \leq \eta^{i}(\alpha, e)$ for any $i \in \mathcal{C}$ and $s^{i}(\alpha, e) \leq s^{i} \leq s^{i}(\alpha, 0)=s_{0}^{i}$ for any $i \notin \mathcal{C}$.
} 
Suppose now that for a bail-in of the above form, some bank $i \in \mathcal{C}$ has an incentive to free-ride, that is, the regulator proceeds with the residual bail-in even without $i$ 's participation. By Condition 1 in Lemma 3.5. this occurs precisely if $W_{N}-W_{\lambda}\left(b, s,\left(0, a^{-i}\right)\right) \geq 0$. If the regulator were able to decrease welfare in the proposed bail-in by this amount for all response vectors, he could eliminate $i$ 's free-riding incentives. It follows from Lemmas 3.8 and 3.9 that welfare in an individually incentive-compatible bail-in $(b, s)$ with contributing banks in $\mathcal{C}$ exceeds the lower bound in $(18)$ by

$$
\lambda \sum_{i \notin \mathcal{C}}\left(s^{i}-s_{0}^{i}\right)^{+}+\lambda \sum_{i \in \mathcal{C}}\left(\eta^{i}(\alpha, 0)-b^{i}\right)^{+} .
$$

Thus, providing subsidies in excess of $s_{0}$ and requesting contributions below $\eta(\alpha, 0)$ are means with which the regulator can decrease or "burn" welfare. Since subsidies in excess of $s_{0}$ and contributions below $\eta(\alpha, 0)$ do not affect the asset price, burning welfare as in 19 does not distort incentives and hence decreases welfare by the same amount in any response vector of the banks. We denote by $\chi_{\mathcal{C}}(\alpha)$ the minimal amount of welfare-burning needed to eliminate free-riding incentives from an individually incentive-compatible bail-in with contributing banks $\mathcal{C}$ that induces asset price $\alpha$. The mathematical definition of $\chi_{\mathcal{C}}(\alpha)$ is somewhat convoluted and deferred to Lemma A.1. In Theorem E.3 of the online appendix, we show that welfare burning is used sparingly in equilibrium.

The analysis above brings us to the characterization of the equilibrium intervention plan. The result states that when the threat is credible, the regulator proposes a bail-in which implements the minimum value burning $\chi_{\mathcal{C}}(\alpha)$ for the optimal choice of $\mathcal{C}$ and $\alpha$. For the sake of reference, we isolate the set of all incentive compatible bail-ins that implement the minimum value burning.

Definition 3.2. Let $z(\alpha):=\alpha \ln (\alpha)$ and let $z^{-1}$ be its inverse on the interval $\left[\frac{1}{\mathrm{e}}, 1\right]$. Define the function $g_{\alpha}(x):=g\left(z^{-1}(z(\alpha)+\gamma x)\right)-g(\alpha)$. Let $\Xi(\mathcal{C}, \alpha)$ denote the set of all bail-ins $(b, s)$ satisfying:

(i) $b^{i}-s^{i} \leq \eta^{i}(\alpha, e)$ for every $i \in \mathcal{C}$,

(ii) $b^{i}=0$ and $s^{i}(\alpha, e) \leq s^{i}$ for every $i \notin \mathcal{C}$,

(iii) $\sum_{i=1}^{n}\left(s_{0}^{i}-s^{i}\right)^{+}+\sum_{i \in \mathcal{C}}\left(b^{i}-b_{0}^{i}\right)^{+}=-\frac{\alpha \ln (\alpha)}{\gamma}$,

(iv) $\lambda \sum_{i=1}^{n}\left(s^{i}-s_{0}^{i}\right)^{+}+\lambda \sum_{i \in \mathcal{C}}\left(\eta^{i}(\alpha, 0)-b^{i}\right)^{+}=\chi_{\mathcal{C}}(\alpha)$,

(v) $\lambda \min \left(b^{i}, b_{0}^{i}\right)+g_{\alpha}\left(\left(b^{i}-b_{0}^{i}\right)^{+}\right) \geq W_{N}-W_{P}+g\left(\alpha_{P}\right)-g(\alpha)+\lambda \sum_{j \in \mathcal{C}} \eta^{j}(\alpha, 0)-\chi_{\mathcal{C}}(\alpha)$ for $i \in \mathcal{C}$.

The contribution of a bank $i \in \mathcal{C}$ to a bail-in affects welfare in two ways. Contributions up to $\eta^{i}(\alpha, 0)$ do not require asset liquidation and increase welfare by an amount $\lambda \eta^{i}(\alpha, 0)$. Contributions that exceed $\eta^{i}(\alpha, 0)$ by an amount $x^{i}$ require asset liquidation and impact the welfare trade-off between taxpayer contributions and asset liquidation by an amount $g_{\alpha}\left(x^{i}\right)$. A contribution of size $\eta^{i}(\alpha, 0)+x^{i}$ by bank $i$ thus has a total impact on welfare of $\lambda \eta^{i}(\alpha, 0)+g_{\alpha}\left(x^{i}\right)$. The first two Conditions in Definition 3.2 state that $(b, s)$ is a feasible, complete bail-in with contributing banks in $\mathcal{C}$. Conditions (iii) and (iv) state that the bail-in induces asset price $\alpha$ and the total amount of welfare burnt is $\chi_{\mathcal{C}}(\alpha)$. Finally, Condition (v) states that there is no free-riding because the 
welfare impact of any bank $i$ (left hand side) is larger than the difference between the welfare losses in a default cascade and in the bail-in. Thus, Conditions (i) and (v) together imply that it is incentive-compatible for any bank $i \in \mathcal{C}$ to accept the proposal.

Theorem 3.10. For any bail-in $(b, s)$, let $\ell(b, s)$ denote the vector of liquidated assets if the proposal is accepted by all banks. For any $\ell$, let $i_{1}(\ell), i_{2}(\ell), \ldots$ denote a decreasing order of banks according to $\eta^{i}(\alpha(\ell), \ell)$. Let $\mathcal{C}(\ell):=\left\{i_{1}(\ell), \ldots, i_{m(\ell)}(\ell)\right\}$, where $m(\ell)$ denotes the smallest integer $k$ such that

$$
W_{P}+\left(g(\alpha(\ell))-g\left(\alpha_{P}\right)\right)-\lambda \sum_{j=1}^{k} \eta^{i_{j}(\ell)}(\alpha(\ell), \ell)<W_{N}
$$

If $W_{P}<W_{N}$, then any subgame Pareto efficient equilibrium outcome is a public bailout with welfare losses $W_{P}$ as specified by Lemma 3.2. If $W_{P} \geq W_{N}$, then there exist generically unique $\mathcal{C}_{*}$ and $\alpha_{*}$ such that in any subgame Pareto efficient equilibrium, a bail-in from the set $\Xi\left(\mathcal{C}_{*}, \alpha_{*}\right)$ is proposed by the regulator and accepted by all banks. ${ }^{26}$ Welfare losses are equal to

$$
W_{E}=W_{P}+\left(g\left(\alpha_{*}\right)-g\left(\alpha_{P}\right)\right)-\lambda \sum_{i \in \mathcal{C}_{*}} \eta^{i}\left(\alpha_{*}, 0\right)+\chi_{\mathcal{C}_{*}}\left(\alpha_{*}\right)
$$

Finally, if $\mathcal{C}_{*}$ is unique, then $\mathcal{C}_{*}=\mathcal{C}(\ell(b, s))$ for all $(b, s) \in \Xi\left(\mathcal{C}_{*}, \alpha_{*}\right)$.

As we have highlighted before, a bail-in can be organized in equilibrium if and only if the regulator's no-intervention threat is credible. The set of contributing banks $\mathcal{C}_{*}$ is the set of banks that are most exposed to contagion at the equilibrium asset price $\alpha_{*}$ : after choosing a set of liquidation decisions $\ell$ that induces $\alpha_{*}$, the regulator adds banks into the bail-in consortium in decreasing order of their incentive compatible contributions $\eta(\alpha(\ell), \ell)$ until welfare losses in the bail-in are lower than in the default cascade. This occurs after adding the $m(\ell)$ most exposed banks. Because of the no-free-riding constraint of Lemma 3.5, no more contributors can be added after that: any additional bank would know that even without its contribution, the regulator will proceed with the residual consortium and, hence, that bank has no incentive to participate.

The asset price and the welfare losses are generically unique in equilibrium. The set of liquidation decisions and the bail-in proposal, however, are not unique in general. Similarly as in the public bailout, welfare depends on the liquidation by banks only through the total amount that is being liquidated. This gives the regulator some leeway on how to induce asset price $\alpha_{*}$. The only restriction on liquidation by an individual bank is the fifth condition in Definition 3.2, specifying the minimal liquidation amount by bank $i$ for the no-free-riding condition to hold.

In Appendix E we highlight the relationship between the equilibrium asset price and the amount of welfare burnt in equilibrium: in many situations, the regulator will avoid burning welfare in equilibrium and instead choose to induce an asset price, at which the contributions by individual banks are sufficiently large to deter free-riding without burning welfare.

\footnotetext{
${ }^{26}$ Generically unique means that it is unique for an open and dense set of model parameters.
} 


\section{Remark 3.2.}

1. Banks are willing to make larger contributions to a bail-in that guarantees a higher asset price, hence the regulator's indifference price increases as more banks are included into the bail-in consortium. In particular, $\alpha_{*} \geq \min \left(\alpha_{\text {ind }}, 1\right)$.

2. If the regulator had the power to commit to not intervening in the third stage, the equilibrium outcome would improve to $W_{E}$ even if $W_{P}<W_{N}$. Commitment power would thus improve social welfare by $\left(W_{P}-W_{E}\right) 1_{\left\{W_{P}<W_{N}\right\}}$.

\section{Shocks, Asset Illiquidity, and Total Throughput}

In this section, we analyze the dependence of equilibrium quantities including asset prices, awarded subsidies, credibility, and welfare losses on the banks' balance sheet parameters, the network structure, and the recovery rates from asset liquidation and bankruptcy procedures ${ }^{27}$

\subsection{Optimal Bailout}

Since every bank is rescued in a complete bailout, there are no bankruptcy costs and no losses that depend on the network structure. Asset prices, subsidies, and welfare losses in the complete bailout are thus independent of $\beta$ and $\pi$. The dependence of the welfare-maximizing bailout on the price elasticity $\gamma$, the welfare cost $\lambda$ of a taxpayer dollar, and the size $s_{0}$ of the banks' shortfall is described in the following result and illustrated graphically in Figure 2 .

Lemma 4.1. There exist constants $0<\gamma_{1}<\gamma_{2}$ and $0<\lambda_{1}<\lambda_{2}$ such that:

(i) The asset price $\alpha_{P}$ is decreasing for $\gamma \leq \gamma_{1}$, increasing for $\gamma \in\left[\gamma_{1}, \gamma_{2}\right]$, and equal to 1 for $\gamma \geq \gamma_{2}$. Subsidies and welfare losses are increasing for $\gamma \leq \gamma_{2}$ and constant for $\gamma \geq \gamma_{2}$.

(ii) The asset price $\alpha_{P}$ and subsidies are constant for $\lambda \leq \lambda_{1}$, decreasing for $\lambda \in\left[\lambda_{1}, \lambda_{2}\right]$, and constant again for $\lambda \geq \lambda_{2}$. Welfare losses are increasing in $\lambda$.

(iii) The asset price $\alpha_{P}$ is non-increasing in $s_{0}^{i}$ for any bank $i$. Subsidies and welfare losses are increasing in $s_{0}^{i}$ for any bank $i$.

When the price elasticity $\gamma$ is low, liquidation has a small impact on the asset price and it is welfare maximizing for the regulator to provide only the minimal amount of subsidies necessary to ensure solvency of all banks in the system. Under this minimal-intervention policy, the price of the asset falls as its elasticity increases. The resulting decrease of the banks' equity value requires larger subsidies to restore the system to a going concern. When the price elasticity rises above the threshold $\gamma_{1}$, the regulator switches from the minimal-intervention policy to a policy that trades off

\footnotetext{
${ }^{27}$ The results in this section are presented under the assumption that $\gamma>0, e^{i}>0$ for every bank $i$, and that there is at least one fundamentally defaulting bank. Without this assumption, the results in this section hold when strict monotonicity is replaced by weak monotonicity.
} 

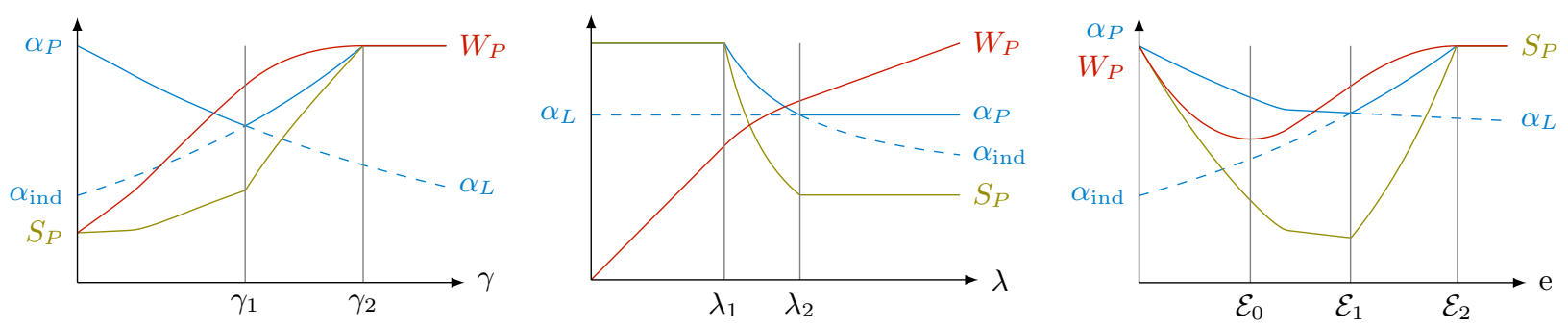

Figure 2: The three charts show the dependence of the optimal bailout on $\gamma, \lambda$, and $e$ for a calibrated model of a dense financial network using the 2018 data of the EBA stress test ${ }^{28}$ The asset price $\alpha_{P}=\min \left(\max \left(\alpha_{L}, \alpha_{\text {ind }}\right), 1\right)$ is shown in solid blue lines; the minimal-intervention asset price $\alpha_{L}$ and the indifference price $\alpha_{\text {ind }}$ are shown in dashed blue lines. The total subsidies $S_{P}$ awarded and welfare losses $W_{P}$ are shown in yellow and red, respectively, normalized to fit the same scale. By Lemma 4.1 the dependence patterns are the same for arbitrary financial networks.

asset liquidation and taxpayer contributions to maintain the indifference price $\alpha_{\text {ind }}$. As liquidation becomes more costly, the regulator has to provide additional subsidies to maintain the indifference price, which has a positive net effect on the asset price. In the interval $\left[\gamma_{1}, \gamma_{2}\right]$, non-defaulting banks thus benefit from additional market illiquidity - at the cost of taxpayer contributions - since their equity value increases with the asset price. Above the threshold $\gamma_{2}$, liquidation is so costly that the regulator covers the banks' entire shortfall $s_{0}$ to avoid any liquidation.

Covering the entire shortfall with taxpayer contributions is optimal if the welfare cost of taxpayer contributions is below the threshold $\lambda_{1}$. Above the threshold, the regulator decreases the size of subsidies as $\lambda$ increases to attain the indifference price. A decrease in subsidies will lead to a decrease in the asset price until, at level $\lambda_{2}$, the regulator provides only the minimal subsidies necessary.

Finally, subsidies and welfare losses are increasing in the shortfall $s_{0}$ of the banks, which can be understood as a measure of the size of the initial shock. The asset price is strictly decreasing in the size of the shock where it exceeds the indifference price and it is constant otherwise. Since the indifference price does not depend on the size of the shock, a threshold where the transition takes place may not exist: the asset price may be strictly decreasing or it may be constant for any shock size.

Lemma 4.1 divides the space of parameters into regions where the regulator provides the minimal amount of subsidies $\left(\alpha_{P}=\alpha_{L}\right)$, where the regulator trades off taxpayer contributions with asset liquidation $\left(\alpha_{P}=\alpha_{\text {ind }}\right)$, and where liquidation is so costly that the regulator covers the entire shortfall with subsidies $\left(\alpha_{P}=1\right)$. To describe the same phenomenon in terms of the size of illiquid assets held by the banks, we need the notion of separating manifolds, which generalizes thresholds to higher dimensions. Separating manifolds, like separating hyperplanes, divide $\mathbb{R}_{+}^{n}$ monotonically into two regions, but they can accommodate a non-linear boundary.

Definition 4.1. A separating manifold $\mathcal{X}$ is a subset of $\mathbb{R}^{n}$ that admits a global parametrization $f^{i}$ along every coordinate $i=1, \ldots, n$ such that for every $x \notin \mathcal{X}$, either $f^{i}\left(x^{-i}\right)>x^{i}$ for each $i=$ $1, \ldots, n$ or $f^{i}\left(x^{-i}\right)<x^{i}$ for each $i=1, \ldots, n$. We write $x<\mathcal{X}$ if $x^{i}<f^{i}\left(x^{-i}\right)$ for each $i$ and we write $x \leq \mathcal{X}$ if $x<\mathcal{X}$ or $x \in \mathcal{X}$. We define $>$ and $\geq$ analogously.

\footnotetext{
${ }^{28}$ Unlike the data calibration procedure for Section 6 described in Appendix D we do not shut down the price-mediated contagion channel for Figures 2 and 4 Instead, we set $90 \%$ of outside assets reported by banks as illiquid and $10 \%$ as cash.
} 
Lemma 4.2. There exist separating manifolds $0<\mathcal{E}_{0} \leq \mathcal{E}_{1}<\mathcal{E}_{2}$ such that:

(i) For a fundamentally defaulting bank $i \in \mathcal{F}$, the asset price $\alpha_{P}$ and subsidies are decreasing in $e^{i}$ for $e \leq \mathcal{E}_{1}$, increasing in $e^{i}$ for $\mathcal{E}_{1} \leq e \leq \mathcal{E}_{2}$, and constant for $e \geq \mathcal{E}_{2}$. Welfare losses are decreasing in $e^{i}$ for $e \leq \mathcal{E}_{0}$, increasing in $e^{i}$ for $\mathcal{E}_{0} \leq e \leq \mathcal{E}_{1}$, and constant for $e^{i} \geq \mathcal{E}_{2}$.

(ii) For a non-fundamentally defaulting bank $i \notin \mathcal{F}$, the asset price $\alpha_{P}$ and subsidies are constant in $e^{i}$ for $e \leq \mathcal{E}_{1}$ or $e \geq \mathcal{E}_{2}$ and they are increasing in $e^{i}$ for $\mathcal{E}_{1} \leq e \leq \mathcal{E}_{2}$. Welfare losses are increasing in $e^{i}$ for $e \leq \mathcal{E}_{2}$ and constant for $e^{i} \geq \mathcal{E}_{2}$.

Similarly to Lemma 4.1, Lemma 4.2 divides the vector of illiquid asset holdings $e$ into three regions, where it is optimal for the regulator to provide minimal assistance $\left(e \leq \mathcal{E}_{1}\right)$, where the regulator trades off taxpayer contributions with asset liquidation $\left(\mathcal{E}_{1} \leq e \leq \mathcal{E}_{2}\right)$, and where the regulator prevents all liquidation $\left(e \geq \mathcal{E}_{2}\right)$. Generally speaking, an increase in the sum of illiquid assets held by the entire system has a similar impact on the optimal bailout as an increase in the price elasticity because the same amount of liquidation leads to larger fire-sale losses. However, there is a subtle difference below $\mathcal{E}_{1}$, where minimal subsidies are optimal: A fundamentally defaulting bank $i$ cannot cover its shortfall by liquidating its assets. The regulator is thus forced to cover a large portion of the shortfall even if the marginal welfare cost of taxpayer contributions is higher than the marginal welfare impact of asset liquidation. As $e^{i}$ increases, bank $i$ is able to cover a larger portion of its shortfall by liquidating its assets, which reduces the size of the minimal subsidies required. Below the threshold $\mathcal{E}_{0}$, the benefits of the reduced size of subsidies outweighs additional losses from fire sales, leading to an overall decrease in welfare losses. Note that $\mathcal{E}_{0}$ may be strictly lower than $\mathcal{E}_{1}$ if the aggregate amount of the illiquid asset held by the other banks in the system is large. See the right panel in Figure 2 for a graphical illustration.

\subsection{Credibility}

For a given set of parameters, the threat is either credible or not. To analyze how the credibility depends on underlying variables, we study the difference $W_{P}-W_{N}$ between the welfare losses in the optimal bailout and the default cascade. This measures how close to being credible the threat is. We say that the credibility of the threat is increasing or decreasing in a parameter if $W_{P}-W_{N}$ is, respectively, increasing and decreasing in that parameter.

A critical measure for the sensitivity analysis is the total throughput of a defaulting bank to the solvent members of the economy. We define it as follows. Abbreviate $\mathcal{D}_{N}=\mathcal{D}\left(p_{N}, \ell_{N}, \alpha_{N}\right)$, let $\mathcal{C}_{N} \subseteq \mathcal{D}_{N}$ denote the set of defaulting banks which repay their senior creditors in full, and let $\mathcal{I}_{N}$ denote the set of illiquid but solvent banks. For two sets of banks $\mathcal{S}$ and $\mathcal{C}$, let $\pi^{\mathcal{S}, \mathcal{C}}$ denote the submatrix of $\pi$ with rows and columns corresponding to banks in $\mathcal{S}$ and $\mathcal{C}$, respectively. The throughput of a bank $i \in \mathcal{C}_{N}$ to a set of banks $\mathcal{S}$ is

$$
\theta_{\mathcal{S}}^{i}(\beta, \pi):=\sum_{j \in \mathcal{S} \backslash \mathcal{D}_{N}} \pi^{\{j\} \mathcal{C}_{N}}\left(I-\beta \pi^{\mathcal{C}_{N}, \mathcal{C}_{N}}\right)^{-1} \rho_{i}^{\mathcal{C}_{N}}+\beta \sum_{j \in \mathcal{S} \cap \mathcal{D}_{N} \backslash \mathcal{C}_{N}} \pi^{\{j\} \mathcal{C}_{N}}\left(I-\beta \pi^{\mathcal{C}_{N}, \mathcal{C}_{N}}\right)^{-1} \rho_{i}^{\mathcal{C}_{N}} .
$$




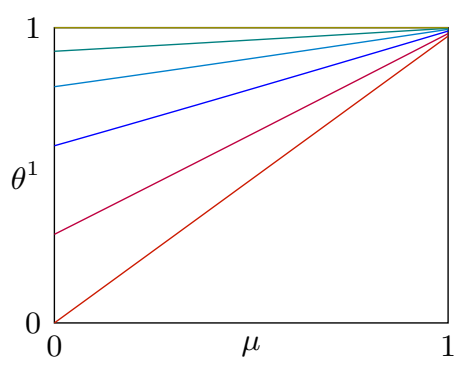

$$
\begin{aligned}
& \beta=1 \\
& \beta=0.92 \\
& \beta=0.8 \\
& \beta=0.6 \\
& \beta=0.3 \\
& \beta=0
\end{aligned}
$$

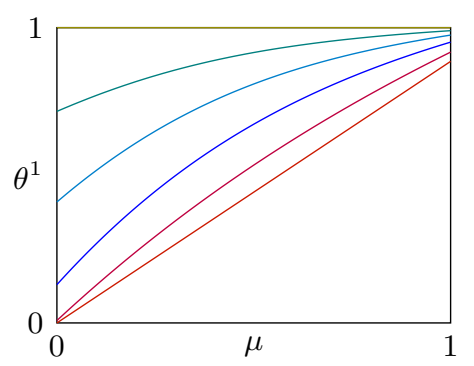

Figure 3: Let $\pi_{c}$ and $\pi_{r}$ denote the complete and the ring network, respectively. The two charts show the total throughput $\theta^{1}\left(\beta, \pi_{\mu}\right)$ of bank 1 in the network $\pi_{\mu}=\mu \pi_{c}+(1-\mu) \pi_{r}$ for various levels of $\beta$, when $\mathcal{C}_{N}=\mathcal{D}_{N}=\{1,2\}$ and $\mathcal{C}_{N}=\mathcal{D}_{N}=\{1,2,3,4,5\}$ in the left and right panel, respectively.

where $\rho_{i}^{\mathcal{C}_{N}}$ denotes the unit vector in $\mathbb{R}^{\mathcal{C}_{N}}$ in the direction of $i$. The total throughput of bank $i \in \mathcal{C}_{N}$ is then defined as $\theta^{i}(\beta, \pi):=\theta_{\{1, \ldots, N\}}^{i}(\beta, \pi)$. For a bank $i \in \mathcal{D}_{N} \backslash \mathcal{C}_{N}$, we set $\theta^{i}(\beta, \pi):=1$.

The total throughput of bank $i$ is a measure of the exposure of solvent junior creditors (first term) and senior creditors (second term) to a shock hitting bank $i$. It thus quantifies the potential for spillover losses triggered by defaults. For a bank $i \in \mathcal{C}_{N}$, it captures the amplification of losses due to negative feedback loops between defaulting banks through the Leontief matrix $\left(I-\beta \pi^{\mathcal{C}_{N}, \mathcal{C}_{N}}\right)^{-1}=$ $\sum_{k=0}^{\infty}\left(\beta \pi^{\mathcal{C}_{N}, \mathcal{C}_{N}}\right)^{k}$ : term $k$ in the sum corresponds to the propagation of losses through liability chains in $\mathcal{C}_{N}$ of length $k$. After accounting for feedback effects and bankruptcy losses, the exposure of a solvent creditor to a shock on bank $i$ 's assets is $\pi^{j i}$ for a solvent bank $j$ and $\beta \pi^{j i}$ for the senior creditors of a bank $j \in \mathcal{D}_{N} \backslash \mathcal{C}_{N} 29$ The total throughput is a normalized measure for the rate of spill-over losses that condenses all the network information needed to determine the credibility of the regulator's threat, as specified by the following lemma.

Lemma 4.3. The total throughput of any bank is non-decreasing in $\beta$ and it takes values in $[0,1]$. Conditional on the banks' levels of solvency (the sets $\mathcal{D}_{N}, \mathcal{C}_{N}$, and $\mathcal{I}_{N}$ ) and the total value of their claims on solvent banks, $W_{P}-W_{N}$ depends on $\pi$ only through $\sum_{i \in \mathcal{C}_{N}} \theta_{\mathcal{I}_{N}}^{i}(\beta, \pi)$ and $\sum_{i \in \mathcal{C}_{N}} \theta^{i}(\beta, \pi)$.

Observe from 20 that the throughput depends only on the network structure, the location of the shocked bank(s) within the network, the connections of the shocked banks to other defaulting banks, as well as the recovery rate $\beta$. Conditional on the banks' levels of solvency, it does not, however, depend on the asset price or the banks' balance sheet quantities $L, c, w$, and $e$. The throughput is increasing in the connectivity between defaulting banks as illustrated in Figure $3^{30}$ We are now ready to determine the dependency of the credibility on the model parameters.

\section{Lemma 4.4.}

(i) The credibility of the threat is increasing in $\lambda$.

\footnotetext{
${ }^{29}$ The total throughput of bank $i \in \mathcal{C}_{N}$ is related to the bank's Bonacich centrality $B^{i}=\mathbf{1}_{\mathcal{C}_{N}}^{\top}\left(I-\beta \pi^{\mathcal{C}_{N}, \mathcal{C}_{N}}\right)^{-1} \rho_{i}^{\mathcal{C}_{N}}$, which captures the total amplification of losses through feedback loops in $\mathcal{C}_{N}$. The total throughput additionally takes into account how the losses are distributed among the creditors. It is important to note that the Bonacich centrality may diverge to $\infty$ if $\beta \rightarrow 1$, whereas our notion of total throughput is bounded on the interval $[0,1]$.

${ }^{30}$ The symmetric complete network $\pi_{c}$ with $\pi_{c}^{i j}=1_{\{i \neq j\}} /(n-1)$ is the least concentrated network structure. The ring network $\pi_{r}$ with $\pi_{r}^{i j}=1_{\{j=i+1 \bmod n\}}$ is the sparsest network structure as measured by the Gini index; see Hurley and Rickard (2009).
} 

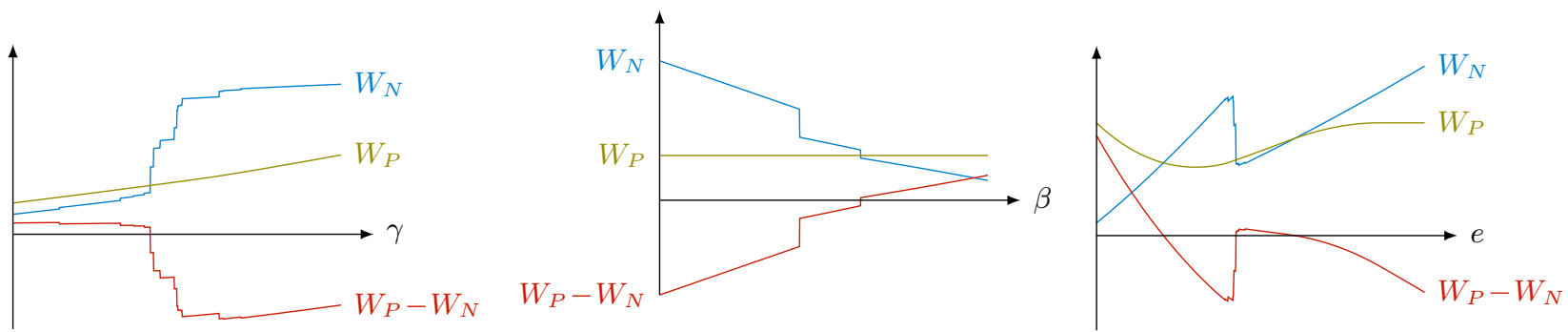

Figure 4: The three charts show the dependence of the optimal bailout on $\gamma, \beta$, and $e$ for a calibrated model of a dense financial network using the 2018 data of the EBA stress test. In the right chart, we scale the size of illiquid assets held by all banks simultaneously, leading to a cluster of thresholds $e_{*}^{i}$, where each bank $i$ becomes solvent and the credibility of the threat increases.

(ii) The credibility of the threat is non-decreasing in $\beta$ and it is strictly increasing on the set $\left\{\beta \mid\right.$ there exists $i \in \mathcal{D}_{N}(\beta)$ with $\left.\theta^{i}(\beta, \theta)>0\right\}$.

(iii) The credibility of the threat is decreasing in $\gamma$ for $\gamma \geq \gamma_{2}$. For $\gamma \leq \gamma_{2}$, an increase in $\gamma$ leads to downward discontinuities in $W_{P}-W_{N}$ when additional banks default. The marginal change of $W_{P}-W_{N}$ at continuity points in $\left[0, \gamma_{2}\right]$ is decreasing in $\beta$ and $\theta^{i}(\beta, \pi)$ for every defaulting bank $i$; see Lemma H.14 in the online appendix for the precise expression.

(iv) If $(1+\lambda) \beta \leq 1$ or $\mathcal{D}_{N}=\mathcal{C}_{N}$, then for each $i$ and fixed $e^{-i}$, there exists $e_{*}^{i}$ such that the credibility of the threat is decreasing in $e^{i}$ everywhere except at $e_{*}^{i}$ where $W_{P}-W_{N}$ has an upward discontinuity 31

As the welfare cost $\lambda$ of taxpayer contribution increases, a bailout becomes more costly and the threat becomes more credible. Similarly, as the recovery rate $\beta$ increases, bankruptcies become less costly and it becomes more credible that the regulator will not intervene. If the throughput of all defaulting banks is 0 , then losses generated by defaulting banks do not spill over to the rest of the system, hence welfare losses are locally constant in $\beta{ }^{32}$ However, if the set of defaulting banks is connected to the rest of the system, then the credibility is strictly increasing in $\beta$.

As the price elasticity $\gamma$ increases, liquidation has a larger knock-down effect on the asset's price. Illiquid banks are able to raise a smaller amount of cash from their sales and, when $\gamma$ crosses a certain threshold, are unable to meet their liabilities. In the absence of intervention, the set of defaulting banks increases at such a threshold of asset illiquidity, causing a downward discontinuity in the credibility of the threat due to bankruptcy losses. Between these discontinuities, two counteracting forces determine the change in credibility: a larger impact on the asset price causes larger liquidation losses in the absence of intervention but also mandates larger subsidies in a bailout. Which effect dominates depends on the recovery rate $\beta$ and the network structure via the total throughput of defaulting banks. As illustrated in Figure 3, the marginal change in credibility is higher in more sparsely connected networks. As seen in the left panel of Figure 4 , the discontinuities typically

\footnotetext{
${ }^{31}$ If $(1+\lambda) \beta>1$ and $\mathcal{D}_{N} \neq \mathcal{C}_{N}$, then one can give a similar condition on the marginal change of $W_{P}-W_{N}$ as in (iii).

${ }^{32}$ Indeed, if all senior creditors are repaid in full and the defaulting banks are only liable to each other but not to the solvent banks in the system, then repayments are just a redistribution of net zero wealth among defaulting banks.
} 
dominate the continuous changes in the credibility. When $\gamma \geq \gamma_{2}$, subsidies in the bailout are constant and the credibility decreases.

An increase in the amount of illiquid asset held by any bank increases the system's exposure to fire-sale losses both in the optimal bailout and in the default cascade. Because fire-sale losses are partially mitigated when a bailout is implemented, the sensitivity of welfare losses to higher asset holdings is stronger in absence of intervention than in a bailout. Therefore, the credibility of the threat is decreasing in $e^{i}$ for each bank $i$ except at the threshold $e_{*}^{i}$, where bank $i$ changes from being insolvent for $e^{i}<e_{*}^{i}$ to being solvent for $e^{i} \geq e_{*}^{i}$; see the right panel in Figure 4.

The following result shows how the credibility of the threat changes with the size of the initial shock. For the credibility analysis, we write $c=c_{0}-\varepsilon$ as the sum of cash kept in period $t=0$ and the realization of short-term returns on the illiquid assets in period $t=1$, where $\varepsilon$ is interpreted as the size of the shock to those returns. In contrast, the public bailout depends on the size $\varepsilon$ of the initial shock only through the shortfall $s_{0}$, hence Lemma 4.1 simply states the dependency on $s_{0}$.

Lemma 4.5. For each bank $i$, there exist $0<\varepsilon_{1}^{i}<\varepsilon_{2}^{i} \leq \varepsilon_{3}^{i}$ such that the credibility of the threat is constant for $\varepsilon^{i} \leq \varepsilon_{1}^{i}$, decreasing for $\varepsilon^{i} \in\left[\varepsilon_{1}^{i}, \varepsilon_{2}^{i}\right]$, and increasing for $\varepsilon^{i} \geq \varepsilon_{3}^{i}$. On the interval $\left[\varepsilon_{2}^{i}, \varepsilon_{3}^{i}\right]$, the credibility has only downward discontinuities. The marginal change of $W_{P}-W_{N}$ at continuity points in $\left[\varepsilon_{2}^{i}, \varepsilon_{3}^{i}\right]$ is decreasing in $\beta$ and $\theta^{i}(\beta, \pi)$.

For very small shock sizes, bank $i$ is able to honor its liabilities without liquidating its assets. Welfare losses and the credibility of the threat thus remain unaffected. For small shock sizes in the interval $\left[\varepsilon_{1}^{i}, \varepsilon_{2}^{i}\right]$, bank $i$ has to start liquidating its assets in absence of intervention, but not in the public bailout where all interbank claims are honored. The credibility of the threat is thus decreasing in that interval. For intermediate shock sizes in the region $\left[\varepsilon_{2}^{i}, \varepsilon_{3}^{i}\right]$, banks have insufficient liquidity to repay their liabilities both in the bailout and in the no-intervention outcome. Whether this leads to a larger increase of welfare losses without intervention than in a bailout - and hence to a decrease in credibility - depends on the recovery rate $\beta$, and the total throughput of bank $i$ in the default cascade. Finally, for large shock sizes $\varepsilon^{i} \geq \varepsilon_{3}^{i}$, bank $i$ does not make any payment to its junior creditors in the default cascade, hence any marginal increase in the shock is not amplified through the network anymore. Consequently, the credibility is increasing in the shock size.

We conclude this section with the following result, which highlights that the credibility of the threat is the most important determinant when comparing welfare losses between two networks.

Lemma 4.6. For fixed $L, e, c, w, \gamma, \beta$, equilibrium welfare losses after intervention are smaller in network $\pi_{1}$ than in network $\pi_{2}$ if the regulator's threat is credible in $\pi_{1}$ but not in $\pi_{2}$.

Indeed, if the regulator's threat is credible in network $\pi^{1}$ but not in network $\pi^{2}$, Theorem 3.10 implies that $W_{E}\left(\pi^{1}\right)<W_{N}\left(\pi^{1}\right) \leq W_{P}=W_{E}\left(\pi^{2}\right)$. If the threat fails to be credible in both networks, the only available option for the regulator in either network is a public bailout. Therefore, equilibrium welfare losses are identical in both networks. In Section 6, we compare welfare losses when the threat is credible in both networks. We do that numerically, using a data set from the European Banking Authority's 2018 stress test. 


\section{Optimal Intervention with Partial Rescues}

In this section, we extend the baseline model of Section 3 by enlarging the strategy space of the government from complete rescues to bail-ins and bailouts that may rescue only a subset of the banks. We also refer to these interventions as partial interventions or partial rescues. This analysis reveals the additional forces that contribute to the formation of bail-ins when the regulator does not necessarily rescue every bank in the system. To simplify the analysis and the exposition of our results, we assume a perfectly elastic asset price, that is, we set $\gamma=0$. This is equivalent to assuming that banks do not hold any illiquid asset and all non-interbank claims are cash assets.

\subsection{Public Bailout}

Without the participation of the banks, the regulator minimizes welfare losses over all possible sets of banks he could bail out. The first lemma describes this minimization procedure.

Lemma 5.1. For any set of banks $\mathcal{B}$, let $p(\mathcal{B})$ be the greatest fixed-point of

$$
p^{i}= \begin{cases}L^{i} & \text { if } i \in \mathcal{B} \text { or } c^{i}+\sum_{j=1}^{n} \pi^{i j} p^{j} \geq L^{i}+w^{i}, \\ \left(\beta\left(c^{i}+(\pi p)^{i}\right)-w^{i}\right)^{+} & \text {otherwise. }\end{cases}
$$

Define the vector of subsidies $s(\mathcal{B})$ by setting $s^{i}(\mathcal{B}):=\left(L^{i}+w^{i}-c^{i}-\sum_{j=1}^{n} \pi^{i j} p^{j}(\mathcal{B})\right)^{+}$for $i \in \mathcal{B}$ and $s^{i}(\mathcal{B})=0$ otherwise. Let $\mathcal{B}_{P}:=\arg \min _{\mathcal{B}} W_{\lambda}(s(\mathcal{B}))$. A welfare-maximizing partial bailout awards subsidies from the set $\mathcal{S}_{P}:=\left\{s\left(\mathcal{B}_{*}\right) \mid \mathcal{B}_{*} \in \mathcal{B}_{P}\right\}$ and attains welfare losses $W_{P}^{*}:=\min _{\mathcal{B}} W_{\lambda}(s(\mathcal{B}))$.

The bailout $s(\mathcal{B})$ is the welfare-maximizing bailout among all bailouts that rescue banks in $\mathcal{B}$ by giving subsidies only to banks in $\mathcal{B}$. The regulator thus maximizes welfare by optimally selecting which banks to subsidize. Generically, $\mathcal{B}_{P}$ is a singleton, that is, welfare is maximized for a unique set of banks to be bailed out. Note that in Lemma 5.1, we do not preclude the possibility that the optimal partial bailout rescues no banks at all. In that case, the optimal "bailout" is the default cascade. The following result describes the structure of the partial bailout by characterizing conditions under which it is optimal not to rescue a certain set of banks. Those conditions depend on the shortfall $S(\mathcal{B})$ and the capital buffer $C(\mathcal{B})$ in the bailout $s(\mathcal{B})$, defined as follows:

$$
S(\mathcal{B}):=(L+w-c-\pi p(\mathcal{B}))^{+}, \quad C(\mathcal{B}):=(c+\pi p(\mathcal{B})-w-L)^{+} .
$$

For the sake of brevity, we set $\delta(\mathcal{B})=\delta(p(\mathcal{B}))$ and $\mathcal{D}(\mathcal{B})=\mathcal{D}(p(\mathcal{B}))$, and denote by $\mathcal{S}(\mathcal{B})=\mathcal{D}(\mathcal{B})^{c}$ the set of solvent banks in the bailout $s(\mathcal{B})$, where $A^{c}$ denotes the complement of the set $A$.

Lemma 5.2. For any two sets of banks $\mathcal{B}, \mathcal{B}^{\prime}$ such that $\mathcal{B}^{\prime} \subseteq \mathcal{B}$, let $\zeta:=\pi\left(p(\mathcal{B})-p\left(\mathcal{B}^{\prime}\right)\right)$ and $\mathcal{R}:=\mathcal{D}\left(\mathcal{B}^{\prime}\right) \backslash \mathcal{D}(\mathcal{B})$. Then $W_{\lambda}\left(\mathcal{B}^{\prime}\right)<W_{\lambda}(\mathcal{B})$ if and only if

$$
\sum_{i \in \mathcal{R}} S^{i}\left(\mathcal{B}^{\prime}\right)+\frac{\lambda}{1+\lambda} \sum_{i \in \mathcal{S}(\mathcal{B})} \min \left(\zeta^{i}, C^{i}(\mathcal{B})\right)>\sum_{i \in \mathcal{R}} \delta^{i}\left(\mathcal{B}^{\prime}\right)+\sum_{i \in \mathcal{D}(\mathcal{B})} \min \left(\beta \zeta^{i}, \delta^{i}\left(\mathcal{B}^{\prime}\right)\right)+\sum_{i \in \mathcal{S}(\mathcal{B})} \zeta^{i}
$$


The left-hand side of 22 captures the benefits for not rescuing banks in $\mathcal{R}$. The first term is proportional to the shortfall of banks in $\mathcal{R}$ if those banks are not rescued. Rescuing them is costly and requires government expenditures in the form of awarded subsidies. The second term captures the potential of solvent banks to absorb losses $\zeta$ caused by the default of banks in $\mathcal{R}$. If solvent banks have sufficiently large capital buffers, these losses can be absorbed by the system and there is less benefit in rescuing the defaulting banks using public money. The right-hand side of (22) captures the benefits of rescuing banks in $\mathcal{R}$. These include the direct benefits to senior creditors (first two terms) and solvent junior creditors (third term) if banks in $\mathcal{R}$ are rescued. Benefits to insolvent banks do not explicitly appear in the expression above because those benefits are passed on to creditors of rescued banks through the repayment of liabilities.

Lemma 5.2 implies that a bank is not rescued in the optimal bailout if it is hit by a very large shock or if such as shock can be well-absorbed by the capital buffer of its creditors. By contrast, a bank is rescued in the optimal bailout if, relative to the size of the exogenous shock, the bank's default causes large losses to its creditors that cannot be absorbed by their capital buffers. Losses are particularly large if the bank's bankruptcy causes a default cascade. If a bank is hit by a large shock and its bankruptcy causes a default cascade, it may be welfare enhancing to rescue the contagiously defaulting banks as opposed to the fundamentally defaulting bank: To rescue the fundamentally defaulting bank, the regulator has to cover the bank's large shortfall entirely using taxpayer money. If he rescues only the contagiously defaulting banks, he can leverage the balance sheet capacity of those banks and cover the residual shortfall only after the capital buffers of the contagiously defaulting banks have been depleted.

From an ex-ante perspective, our result implies that a risky bank $i$ has incentives to borrow from other risky banks in the system so that in case of $i$ 's default, its creditors are likely to be distressed as well. Then, their potential for absorbing the losses induced by bank $i$ is small, increasing the chances that bank $i$ is bailed out. (As we noted in the introduction, in this paper we do not, however, pursue the ex-ante implications of our bail-out strategies.)

\subsection{Banks' Equilibrium Responses}

When the regulator commits to complete bailouts as in Section 3 , the threat towards the banks is binary: a bank's assets are either fully protected or they are exposed to the full extent of the default cascade. This is no longer the case when the government allows for partial bailouts. A bank's assets may be protected to varying degrees in a welfare-maximizing bailout: while some debtors may be rescued and hence the claims towards those banks are protected, other debtors may still default, thereby inducing losses to the remaining banks in the system. Thus, banks may have an incentive to participate in a bail-in even if the regulator cannot credibly threaten a default cascade.

If there is more than one set of banks, whose bailout is welfare-maximizing, the regulator can choose which one to implement. If the regulator announces that he will implement the bailout $s(\mathcal{B})$ when banks fail to cooperate, he threatens to not rescue banks in $\mathcal{B}^{c}$. Because he can credibly implement any optimal bailout after any response vector by the banks, he can "threaten" different 
bailouts to different banks. Consider a financial system with two identical defaulting banks $i$ and $j$, where the welfare-maximizing bailout prescribes the rescue of only one of them. Since rescuing either is a credible action by the regulator, he can threaten the creditors of bank $i$ that, without their participation, he will bail out bank $j$ and vice versa. This is formalized in the following lemma.

Lemma 5.3. Let $(b, s)$ be a feasible bail-in proposal. In an accepting equilibrium a, bank $i$ with $b^{i}>0$ accepts if and only if for some $\mathcal{B}_{i} \in \mathcal{B}_{P}$, the following conditions hold:

1. $W_{\lambda}\left(b, s,\left(0, a^{-i}\right)\right) \geq W_{P}$,

2. $b^{i}-s^{i} \leq \sum_{j=1}^{n} \pi^{i j}\left(\bar{p}^{j}\left(b, s,\left(1, a^{-i}\right)\right)-p^{j}\left(\mathcal{B}_{i}\right)\right)-s^{i}\left(\mathcal{B}_{i}\right)$.

Moreover, if there exists $\mathcal{B}_{*} \in \mathcal{B}_{P}$ such that Condition 2 holds simultaneously for every bank $i$ with $\mathcal{B}_{i}=\mathcal{B}_{*}$, then rejecting equilibria are subgame Pareto efficient only if $s \in \mathcal{S}_{P}$. If there exists no such $\mathcal{B}_{*}$ for any accepting equilibrium, then rejecting equilibria are not subgame Pareto dominated by accepting equilibria.

Condition 1 is analogous to that in the case of complete bailouts and states that a participating bank must not have an incentive to free-ride. Specifically, Condition 1 imposes that without $i$ 's participation, the regulator chooses his preferred outside option over the residual bailin 33 Condition 2 states that bank $i$ 's net contribution to the bail-in $(b, s)$ has to be smaller than or equal to $i$ 's benefits in the bail-in over the threatened bailout $s\left(\mathcal{B}_{i}\right)$. If bank $i$ or $i$ 's debtors are rescued in the bailout $s\left(\mathcal{B}_{i}\right)$, the benefits are lower and $i$ has a lower incentive to contribute. If the optimal bailout of Lemma 5.1 is not unique, the regulator has some room to adjust the threats towards the banks by selecting $\mathcal{B}_{i}$ accordingly. However, if the regulator threatens different bailouts after the rejection by individual banks, the threats can no longer be carried out against all banks simultaneously. Thus, at least one bank is not punished as harshly as it was threatened, hence that bank is strictly better off rejecting the proposal. As a consequence, rejecting equilibria are no longer Pareto dominated by accepting equilibria. The regulator can ensure that his proposal is accepted in equilibrium if he threatens the same bailout after any response from the banks 34 This corresponds to a risk-averse regulator who minimizes welfare losses in the worst-case outcome over all subgame Pareto-efficient continuation equilibria.

\subsection{Optimal Proposal of the Regulator}

In a partial bail-in, the regulator selects both rescued banks and contributors. Because a bank is more willing to contribute to a bail-in that protects its debtors, the two decisions are interconnected.

\footnotetext{
${ }^{33}$ Note that in the case of partial rescues, the preferred outside option is the threatened partial bailout $s\left(\mathcal{B}_{i}\right)$ leading to welfare losses $W_{P}$. By contrast, Lemma 3.5 for complete rescues is formulated under the assumption that the threat is credible, where the regulator's preferred outside option is the default cascade with welfare losses $W_{N}$.

${ }^{34}$ The regulator can choose which welfare-maximizing bailout to implement after any response vector. If it is possible to assign bailouts to response vectors such that at least one bank has an incentive to deviate for any $a \neq(1, \ldots, 1)$, the regulator can prevent the existence of rejecting equilibria. In that case, it does not matter that threats towards different banks cannot be carried out simultaneously. Whether or not rejecting equilibria can be prevented is a combinatorial argument that depends on the number of participating banks, the number of credible bailout threats, and the rank-order of the threats for the individual banks. Since the optimal bailout is generically unique in this framework, this analysis is beyond the scope of the paper.
} 
When the regulator proposes to rescue a set of banks $\mathcal{B}$ and threatens the bailout $s\left(\mathcal{B}_{*}\right)$, the maximal incentive-compatible contribution by bank $i$, or the threat level towards bank $i$, is equal to

$$
\eta_{\mathcal{B}_{*}}(\mathcal{B}):=\min \left(\left(\pi\left(p(\mathcal{B})-p\left(\mathcal{B}_{*}\right)\right)-s\left(\mathcal{B}_{*}\right)\right)^{+}, C(\mathcal{B})\right)
$$

where $C(\mathcal{B})$ is the vector of the banks' capital buffer defined in (21). The notion of threat levels generalizes the credibility of the threat in Section $3^{35}$ If the optimal bailout happens to be the complete bailout, then threat levels towards all banks are zero and, as in Section 3, the regulator cannot incentivize any banks to participate. If the optimal bailout is not the complete bailout, the regulator minimizes welfare losses over which banks to subsidize, taking into account that it affects the contributions he can extract from the private sector. We state the result under the generically satisfied assumption that the optimal bailout is unique. If the welfare-maximizing bailout fails to be unique, the regulator will additionally optimize over which bailouts to threaten.

Theorem 5.4. Suppose that $\mathcal{B}_{P}=\left\{\mathcal{B}_{*}\right\}$. For any set of banks $\mathcal{B}$, denote by $i_{1}(\mathcal{B}), i_{2}(\mathcal{B}), \ldots$ a non-increasing ordering of banks according to $\eta_{\mathcal{B}_{*}}^{i}(\mathcal{B})$. For any integer $k$, define

$$
W^{k}(\mathcal{B}):=W_{\lambda}(s(\mathcal{B}))-\lambda \sum_{j=1}^{k} \eta_{\mathcal{B}_{*}}^{i_{j}(\mathcal{B})}(\mathcal{B})
$$

Let $m(\mathcal{B})$ denote the smallest $k$ for which $W_{\mathcal{B}_{*}}^{k}(\mathcal{B})<W_{P}$ and set

$$
W(\mathcal{B})=\min \left(W^{m(\mathcal{B})}(\mathcal{B}), W_{P}-\lambda \eta_{\mathcal{B}_{*}}^{i_{m(\mathcal{B})+1}(\mathcal{B})}(\mathcal{B})\right)
$$

In the game with partial interventions, the welfare losses in any subgame Pareto-efficient equilibrium are equal to $W_{E}^{*}:=\min _{\mathcal{B}} W(\mathcal{B})$.

As in Theorem 3.10, the no-free-riding constraint drives the regulator to ask for contributions from banks, towards which the threat level is the highest. He includes banks into the bail-in consortium according to the decreasing order $i_{1}(\mathcal{B}), i_{2}(\mathcal{B}), \ldots$ until welfare losses are lower than in the default cascade (left expression in the minimum of (24)). After that, he can only include additional banks into the consortium by burning welfare (right expression in the minimum of (24)) 36

In addition to the above forces that also govern the formation of a complete bail-in, the selection of rescued banks affects the size of the contributions the regulator can demand from the private

\footnotetext{
${ }^{35}$ Because rescues are required to be complete in Section 3 it follows that $\mathcal{B}=\{1, \ldots, n\}$ and hence $p(\mathcal{B})=L$. With complete rescues, the threat level towards the banks is thus either 0 (if the no-intervention threat fails to be credible and hence $p\left(\mathcal{B}_{*}\right)=L$ ) or it is given by $\eta(\alpha(\ell), \ell)$ in 16 for any bail-in that induces asset liquidation $\ell$ (if the no-intervention threat is credible and hence $p\left(\mathcal{B}_{*}\right)=p(\emptyset)=p_{N}$ and $s\left(\mathcal{B}_{*}\right)=0$ ). Here, we highlight the dependence on the set $\mathcal{B}$ of subsidized banks because $\ell=0$ and $\alpha=1$ in this section.

${ }^{36}$ In absence of price-mediated contagion, the order $i_{1}(\mathcal{B}), i_{2}(\mathcal{B}), \ldots$ of banks most exposed to contagion does not depend on the number of banks included in the consortium. The order is fixed for given $\mathcal{B}$ and $\mathcal{B}_{*}$, and this simplifies the characterization of welfare burning in equilibrium: To include an additional bank $i$ into the consortium, the regulator burns $W_{P}-W^{m(\mathcal{B})}(\mathcal{B})$ units of welfare so that without $i$ 's participation, welfare in the residual bail-in and the optimal bailout are identical. Then, bank $i$ does not have an incentive to free-ride. Because banks are decreasingly ordered according to their threat levels, the regulator will consider burning welfare only to include bank $i_{m(\mathcal{B})+1}(\mathcal{B})$.
} 
sector. In equilibrium, the regulator will include few banks into the bail-in consortium, each willing to make a large contribution, rather than many banks with small contributions each. It is, therefore, beneficial to rescue banks which have few large creditors rather than banks with many small creditors. This is formalized in the following lemma. It characterizes the structure of partial equilibrium bail-ins by giving conditions, under which it is optimal not to rescue a certain set of banks. We use the same notation as in Lemma 5.2 and Theorem 5.4 , and additionally denote by $\mathcal{C}(\mathcal{B}):=\left\{i_{1}(\mathcal{B}), \ldots, i_{m(\mathcal{B})}(\mathcal{B})\right\}$ the set of contributing banks towards a rescue of banks in $\mathcal{B}$.

Lemma 5.5. Suppose that $\mathcal{B}_{P}=\left\{\mathcal{B}_{*}\right\}$. For any two sets of banks $\mathcal{B}^{\prime} \subseteq \mathcal{B}$, let $\mathcal{R}:=\mathcal{D}\left(\mathcal{B}^{\prime}\right) \backslash \mathcal{D}(\mathcal{B})$ as in Lemma 5.2. Then $W_{\lambda}\left(s\left(\mathcal{B}^{\prime}\right)\right)-\sum_{i \in \mathcal{C}\left(\mathcal{B}^{\prime}\right)} \lambda \eta^{i}\left(\mathcal{B}^{\prime}\right)<W_{\lambda}(s(\mathcal{B}))-\sum_{i \in \mathcal{C}(\mathcal{B})} \lambda \eta^{i}(\mathcal{B})$ if and only if

$$
\begin{aligned}
\sum_{i \in \mathcal{R}} S^{i}\left(\mathcal{B}^{\prime}\right)+\frac{\lambda}{1+\lambda} \sum_{i \in \mathcal{S}(\mathcal{B})} \min \left(\zeta^{i}, C^{i}(\mathcal{B})\right)+\frac{\lambda}{1+\lambda}\left(\sum_{i \in \mathcal{C}\left(\mathcal{B}^{\prime}\right)} \eta_{\mathcal{B}_{*}}^{i}\left(\mathcal{B}^{\prime}\right)-\sum_{i \in \mathcal{C}(\mathcal{B})} \eta_{\mathcal{B}_{*}}^{i}\left(\mathcal{B}^{\prime}\right)\right) \\
>\sum_{i \in \mathcal{R}} \delta^{i}\left(\mathcal{B}^{\prime}\right)+\sum_{i \in \mathcal{D}(\mathcal{B})} \min \left(\beta \zeta^{i}, \delta^{i}\left(\mathcal{B}^{\prime}\right)\right)+\sum_{i \in \mathcal{S}(\mathcal{B})} \zeta^{i}+\frac{\lambda}{1+\lambda} \sum_{i \in \mathcal{C}(\mathcal{B})} \min \left(\zeta^{i}, \eta_{\mathcal{B}_{*}}^{i}(\mathcal{B})\right) .
\end{aligned}
$$

Similarly to the characterization of the optimal bailout in Lemma 5.2 , the left-hand side of (25) represents the benefits of not rescuing banks in $\mathcal{R}$, whereas the right-hand side represents the benefits of rescuing banks in $\mathcal{R}$. The majority of terms are identical to $(22)$, but there are two key differences. The first is related to the system's ability to absorb losses transmitted from banks in $\mathcal{R}$ when they are not rescued (second term on the left-hand side of (25)). Losses are absorbed either partially or completely by the capital buffers of $\mathcal{R}$ 's creditors. In order to benefit from those capital buffers in a partial bailout, the regulator has an incentive to let banks in $\mathcal{R}$ default. In the bail-in, the regulator benefits from those capital buffers even when he rescues the banks in $\mathcal{R}$ because he can extract larger contributions - up to the amount $\eta(\mathcal{B}) \leq C(\mathcal{B})$ - from contributors in $\mathcal{C}(\mathcal{B})$ (third term on the right-hand side of $(25)$ ). The choice of which banks to rescue is thus network-dependent: If banks in $\mathcal{R}$ have many creditors, only some of them will be included in the consortium $\mathcal{C}(\mathcal{B})$ due to free-riding incentives. Thus, if capital buffers are large and the defaults of banks in $\mathcal{R}$ can be well absorbed, the second term on the left-hand side of 25$]$ is larger than the third term on the right-hand side, constituting a reason not to rescue banks in $\mathcal{R}$. If, however, $\mathcal{R}$ has only a few large creditors, those are likely included in $\mathcal{C}(\mathcal{B})$ and hence the two terms balance out 37 While, in the partial bailout, only the size of the absorbed losses matter, in the partial bail-in the distribution of those losses matters as well because it determines the contributions that can be elicited from the private sector.

The second difference from the case of complete bailouts is the third term on the left-hand side of (25). It captures the structure of rescue consortia in the two alternative bail-ins, stating that it is beneficial to not rescue banks in $\mathcal{R}$ if, by doing so, the regulator does not lose any contributors. Indeed, if the number of banks contributing towards a bail-in rescuing banks in $\mathcal{B}^{\prime}$ is larger than

\footnotetext{
${ }^{37}$ One can show that $\min \left(\zeta^{i}, \eta^{i}(\mathcal{B})\right)=\min \left(\zeta^{i}, C^{i}(\mathcal{B})\right)$ for any bank $i$ with a positive threat level $\eta^{i}\left(\mathcal{B}^{\prime}\right)$ when banks in $\mathcal{R}$ are not rescued. The terms in the sums for each such bank are thus identical.
} 
when rescuing banks in $\mathcal{B}$, i.e., $m\left(\mathcal{B}^{\prime}\right) \geq m(\mathcal{B})$, then this term is positive because $\mathcal{C}\left(\mathcal{B}^{\prime}\right)$ is the set of banks of size $m\left(\mathcal{B}^{\prime}\right)$ that maximizes contributions of size $\eta\left(\mathcal{B}^{\prime}\right)$.

Both Lemmas 5.2 and 5.5 imply that it is beneficial for banks to have only a small number of creditors. To be rescued in a bailout, the bank must cause large contagion effects. To be rescued in a bail-in, the bank's creditors need to be among the largest potential contributors to the rescue consortium. Both are more likely to happen when the losses caused by the bank's default are spread only across a few creditors. Because the regulator prefers sparsely connected networks, it follows that ex-ante incentives of banks are better aligned with the regulator's objective when he allows for partial intervention, rather than when restricting himself to complete rescues only.

Our final result relates the structure of the equilibrium partial bail-in with the structure of the optimal partial bailout.

Lemma 5.6. Let $\mathcal{B} \subseteq \mathcal{B}^{\prime}$ with $W_{\lambda}\left(s\left(\mathcal{B}^{\prime}\right)\right) \leq W_{\lambda}(s(\mathcal{B}))$. Then $W\left(\mathcal{B}^{\prime}\right) \leq W(\mathcal{B})$.

For $\mathcal{B}^{\prime} \in \mathcal{B}_{P}$, Lemma 5.6 shows that it cannot be optimal to rescue a subset of the banks that are bailed out in the threatened bailout: by Lemma 5.3. no bank would have any incentive to contribute to such a bail-in. Lemma 5.6, however, does not imply that all banks from the optimal bailout are rescued in the optimal bail-in, nor is that statement true in general. Condition 2 in Lemma 5.3 shows that, in order to incentive banks to participate, the regulator needs to propose bail-ins that rescue banks that are not bailed out. In the online appendix, we provide a numerical example, in which banks rescued in the optimal bailout are not rescued in the optimal bail-in.

\section{Equilibrium Welfare Losses and Network Structure}

In this section, we analyze the dependency of equilibrium welfare losses on the structure of the interbank network using data from the 2018 stress test of the European Banking Authority (EBA). We elaborate in Appendix $\mathrm{D}$ how we calibrate our model to the data set. To highlight more prominently the impact of the network structure on welfare, we shut down the price-mediated contagion channel for this calibration exercise. This corresponds to setting $\gamma=0$ or, equivalently, assuming that all outside assets are held as cash. Detailed information on bilateral exposures is not publicly available. To compare the relative performance of different network structures, we fit a sparse and a dense network structure $\pi_{s}$ and $\pi_{d}$, respectively, to the data from the EBA stress test. We then analyze the credibility of the regulator's threat and the equilibrium welfare losses as a function of the network structure $\pi_{\mu}:=\mu \pi_{s}+(1-\mu) \pi_{d}$ for $\mu \in[0,1]{ }^{38}$ We generate the dense network $\pi_{d}$ following the maximum entropy method developed by Upper and Worms (2004). This method distributes interbank liabilities as evenly as possible among the counterparties, yielding a complete network. We generate the sparse network $\pi_{s}$ using an iterated greedy algorithm, for which details are provided in

\footnotetext{
${ }^{38}$ Craig and Von Peter (2014) show that the German interbank network has a core-periphery structure: while the 45 large core banks act as intermediaries and have many counterparties, the periphery banks trade only with core banks, but not with each other. The participating banks in the EBA stress test are 36 of the largest banks in Europe, which are all considered core banks. Therefore, we do not aim to estimate a core-periphery network to this data set but rather analyze the impact of a range of network structures of different sparsity on credibility and equilibrium welfare losses for the subnetwork of core banks.
} 

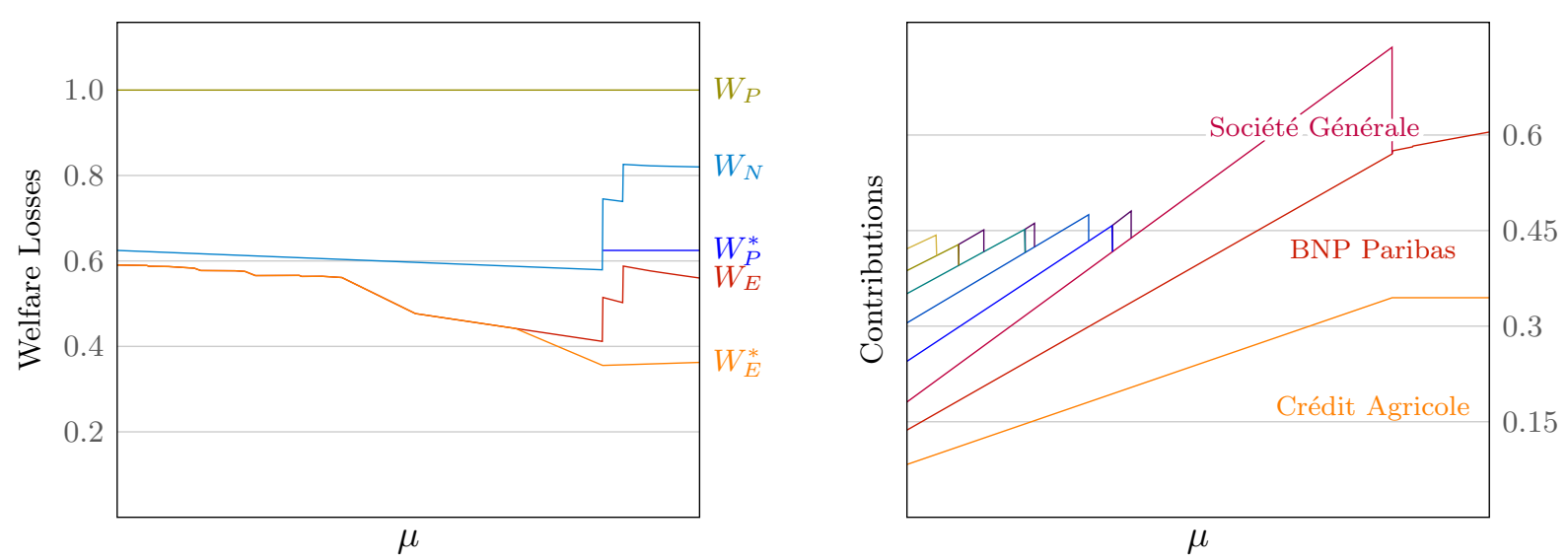

Figure 5: The two plots show how welfare losses under different resolution plans and banks' contributions to the equilibrium bail-in change as a function of the network $\pi_{\mu}=\mu \pi_{s}+(1-\mu) \pi_{d}$ as $\mu$ increases from 0 to 1 . Thus, at the left edge of each plot, the network is equal to a dense network $\pi_{d}$ and it gets progressively sparser as we move to the right until it is equal to a sparse network $\pi_{s}$ at the right edge. Welfare losses and welfare impacts of banks' contributions are shown relative to the welfare losses $W_{P}$ in the complete bailout. Contributions of banks are shown cumulatively so that the contributed amount of a single bank corresponds to the distance between two consecutive lines.

Appendix $D^{39}$ We then apply a shock to the assets of HSBC, Barclays, and Deutsche Bank with a shock size equal to their cash holdings, thereby wiping out the value of their non-interbank assets.

The left plot of Figure 5 shows the impact of the network structure on welfare losses under different resolution plans. As the network becomes sparser, liabilities among banks are more concentrated. This makes contagious defaults in absence of intervention more likely and the corresponding welfare losses $W_{N}$ change discontinuously where this happens. For the chosen size of shocks, the threat is credible in all networks since $W_{N}$ is smaller than welfare losses $W_{P}$ in the complete bailout. This allows the coordination of a complete equilibrium bail-in as described in Theorem 3.10 with welfare losses equal to $W_{E}$. Contributions to the equilibrium bail-in are illustrated in the right plot of Figure 5, where it is evident that they increase as the network becomes sparser because free-riding incentives are reduced. The three main creditors of the shocked banks would suffer large losses without intervention and can, therefore, be incentivized to make large contributions. This leads to a continuous decrease in equilibrium welfare losses as the network gets sparser until we observe contagious defaults in the default cascade: because of the no-free-riding constraints, welfare losses in a bail-in can differ from $W_{N}$ by at most the contribution of any participating bank. Therefore, discontinuous changes in $W_{N}$ are reflected also in the equilibrium welfare losses. Nevertheless, equilibrium welfare losses in the sparsest network are $5.2 \%$ lower than in the most dense network despite the fact that without intervention, they would be $31.2 \%$ larger.

For the chosen shock sizes, there are no contagiously defaulting banks in the most dense network. This illustrates that even if the regulator's threat is more credible in a dense network, equilibrium welfare losses are typically still decreasing in the sparsity of the network because of the reduced freeriding incentives. For larger shock sizes or a more lowly capitalized financial system, we would ob-

\footnotetext{
${ }^{39}$ The resulting network $\pi_{d}$ has 1260 edges, i.e., each of the 36 banks is connected to every other bank, and a normalized Gini index of 0.4556. The network $\pi_{s}$ has 71 edges with a normalized Gini index of 0.9981. See Hurley and Rickard (2009) for a definition of the Gini index. The Gini index is a measure of sparsity, which we normalize to account for the fact that diagonal entries in any relative liability matrix are 0 . The normalized Gini index is 0 for the symmetric complete network and 1 for a ring network.
} 
serve that the credibility improves as the network becomes sparser: consistent with Lemma 3.4 and findings of earlier literature on systemic risk in absence of intervention (e.g., Allen and Gale (2000) and Acemoglu, Ozdaglar and Tahbaz-Salehi (2015)), dense connections amplify a large shock.

Finally, the left panel of Figure 5 illustrates the findings of Section 5, in which we consider interventions that may target only a subset of banks. For the chosen shock sizes, it turns out that in the optimal bailout, the regulator only rescues the contagiously defaulting banks and lets the fundamentally defaulting banks fail. This leads to welfare losses $W_{P}^{*}$, which coincides with $W_{N}$ when there are no contagiously defaulting banks. In the partial equilibrium bail-in $W_{E}^{*}$, it is optimal to rescue every bank since contributions from the private sector can be solicited for the rescue of fundamentally defaulting banks. This is consistent with the predictions of Lemmas 5.2 and 5.5 below. Welfare losses $W_{E}^{*}$ in the partial equilibrium bail-in with partial rescues are $38.5 \%$ lower than in the complete network structure. In this calibrated model, there are 7140 ways of applying idiosyncratic shocks to three banks in the network. Taking the average over all combinations of shocks, equilibrium welfare losses in the sparsest network are lower than in the most dense network

by $13.96 \%$ for complete rescues and by $28.92 \%$ for partial rescues. These results suggest that structural policies aiming at sparsifying the financial network may significantly raise welfare.

\section{Concluding Remarks}

Various initiatives have been undertaken by central governments and monetary authorities, especially after the global financial crisis, to expand resolution plans and tools. Our paper makes a first step towards a systematic analysis of the incentives that govern alternative resolution plans. At the heart of our analysis is the credibility of the regulator's no-intervention (or partial rescue) threat, given the desire of each bank to free-ride on the contributions of the government and of other banks. In a framework with complete rescues, the credibility determines whether a bail-in can be organized in equilibrium or whether the regulator is forced to a bailout. If the regulator is free to propose rescues that save only some banks but not others, threat levels vary across banks and determine the size of incentive-compatible contributions towards a bail-in.

We highlight the main forces impacting the credibility of the no-rescue threat. The threat fails to be credible for a given shock size if and only if the shock is heavily amplified through inefficient asset liquidation, bankruptcy costs, and negative feedback effects between densely connected banks in distress. The impact of the interbank network structure on the credibility is captured by a measure that we call the total throughput of defaulting banks, which reflects the rate at which losses spill over to solvent members of the economy, taking into account feedback effects between defaulting banks. Conditional on the banks' levels of solvency, the total throughput depends entirely on the network structure, giving us a metric to rank the desirability of different network structures.

Our analysis has focused on the design of government interventions, given a particular financial structure. We have shown that when there is a credible bail-in proposal, it is preferable to a bailout because the size of the required public expenditures is lower. While our findings thus explain why public officials have called for substituting bail-ins for bailouts, they also explain why there have 
been so few successful bail-ins: it may not be possible to construct a credible bail-in proposal. Moreover, our analysis shows that selective bail-ins and bailouts, where not all banks are rescued, may be preferable to inclusive policies. In our model, we can precisely define which banks should be rescued, thereby providing the regulator with a rational argument for this selectivity. The partial bailouts of the 2008 financial crisis were widely criticized for being determined more by influence and political connections than by such objective factors.

Our analysis reverses the presumptions concerning the relative desirability of sparse versus dense networks for intermediate shock sizes. This has obvious implications for the design of regulations that affect the network structure. These implications, as well as the broader implications of the interventions that we have described for ex-ante behavior, take us beyond the scope of this limited paper. For now, we simply note two such implications. First, because banks prefer a bailout over a bail-in, they benefit from additional market illiquidity. This implies that ex ante, banks have an incentive to invest into thinly-traded securities. Second, for a bank to be rescued in a partial bail-in, it is beneficial to borrow funds only from a few other banks so that contagion effects in case of the bank's default are highly concentrated. Then, free-riding incentives among creditors are smaller, making the rescue more appealing to the regulator.

This paves the way for future research on endogenous network formation. In such a model, banks anticipate which bail-in consortia are credible for which network structures, and hence choose their counterparties by taking into account their ex-ante expected contributions to an equilibrium bail-in plan. Such an endogenous network formation model adds an additional dimension to the moral hazard literature: through their interbank linkages, banks can control the likelihood of a public bailout as well as the likelihood to be included in the prevailing bail-in. Additionally, it would be desirable to account for the ex-ante risk taking decisions by banks as they maximize the value of their bailout option (as in Acharya and Yorulmazer (2007), Acharya, Shin and Yorulmazer (2011), and Farhi and Tirole (2012) ) ${ }^{40}$ By choosing the riskiness and the liquidity of their investments, banks can further influence the set of available resolution policies. Accounting for ex-ante risk taking behavior will lead to a comprehensive framework for the analysis of welfare maximizing rescue policies.

\section{References}

Acemoglu, D., A. Ozdaglar, and A. Tahbaz-Salehi. 2015. "Systemic Risk and Stability in Financial Networks." American Economic Review, 105(2): 564-608.

Acharya, V.V., and T. Yorulmazer. 2007. "Too Many to Fail-An Analysis of Time-Inconsistency in Bank Closure Policies." Journal of Financial Intermediation, 16(1): 1-31.

Acharya, V.V., H.S. Shin, and T. Yorulmazer. 2011. "Crisis Resolution and Bank Liquidity." Review of Financial Studies, 24(6): 2166-2205.

Allen, F., and D. Gale. 2000. "Financial Contagion." Journal of Political Economy, 108(1): 1-33.

Basel-III. 2013. "The Liquidity Coverage Ratio and liquidity risk monitoring tools." Basel Committee on Banking Supervision.

\footnotetext{
${ }^{40}$ The former two papers focus on endogenous (non-interbank) asset correlation and liquidity risks that arise when banks make their investment decisions in anticipation of a bailout, whereas the latter describes how the bailout option leads to excessive risk taking.
} 
Battiston, S., D. Delli Gatti, M. Callegati, B. Greenwald, and J.E. Stiglitz. 2012. "Default Cascades: When Does Risk Diversification Increase Stability?" Journal of Financial Stability, 8(3): 138149.

Benoit, S., J.-E. Colliard, C. Hurlin, and C. Pérignon. 2017. "Where the Risks Lie: A Survey on Systemic Risk." Review of Finance, 21(1): 109-152.

Bernard, B., A. Capponi, and J.E. Stiglitz. 2019. "Online Appendix to "Bail-ins and Bail-outs: Incentives, Connectivity, and Systemic Stability", available at http://benjamin-bernard.com."

Boissay, F. 2006. "Credit Chains and the Propagation of Financial Distress, available at http://ssrn.com/abstract_id=872543." ECB Working Paper Series, No. 573.

Cabrales, A., P. Gottardi, and F. Vega-Redondo. 2017. "Risk-Sharing and Contagion in Networks." Review of Financial Studies, 30(9): 3086-3127.

Capponi, A., P.C. Chen, and D. Yao. 2016. "Liability Concentration and Systemic Losses in Financial Networks." Operations Research, 64(5): 1121-1134.

Castiglionesi, F. 2007. "Financial Contagion and the Role of the Central Bank." Journal of Banking and Finance, 31(1): 81-101.

Chari, V.V., and P.J. Kehoe. 2016. "Bailouts, Time Inconsistency, and Optimal Regulation: A Macroeconomic View." American Economic Review, 106(9): 2458-2493.

Cifuentes, R., G. Ferrucci, and H.S. Shin. 2005. "Liquidity Risk and Contagion." Journal of the European Economic Association, 3(2): 556-566.

Cooper, R., and K. Nikolov. 2018. "Government Debt and Banking Fragility: The Spreading of Strategic Uncertainty." International Economic Review, 59(4): 1905-1925.

Craig, B., and G. Von Peter. 2014. "Interbank Tiering and Money Center Banks." Journal of Financial Intermediation, 23(3): 322-347.

Duffie, D. 2010. "The Failure Mechanics of Dealer Banks." Journal of Economic Perspectives, 24(1): 51-72.

Duffie, D., and C. Wang. 2017. "Efficient Contracting in Network Financial Markets." Working Paper, Graduate School of Business, Stanford University.

Eisenberg, L., and T.H. Noe. 2001. "Systemic Risk in Financial Systems." Management Science, 47(2): 236-249.

Elliott, M., B. Golub, and M.O. Jackson. 2014. "Financial Networks and Contagion." American Economic Review, 104(10): 3115-3153.

Ellul, A., C. Jotikasthira, and C.T. Lundblad. 2011. "Regulatory Pressure and Fire Sales in the Corporate Bond Market." Journal of Financial Economics, 101(3): 596-620.

Elsinger, H., A. Lehar, and M. Summer. 2006. "Risk Assessment for Banking Systems." Management Science, 52(9): 1301-1314.

Erol, S. 2018. "Network Hazard and Bailouts." Working paper, University of Pennsylvania.

Farhi, E., and J. Tirole. 2012. "Collective Moral Hazard, Maturity Mismatch, and Systemic Bailouts." American Economic Review, 102(1): 60-93.

Freixas, X., B.M. Parigi, and J.-C. Rochet. 2000. "Systemic Risk, Interbank Relations and Liquidity Provision by the Central Bank." Journal of Money Credit and Banking, 32(3): 661-638.

Gai, P., A. Haldane, and S. Kapadia. 2011. "Complexity, Concentration and Contagion." Journal of Monetary Economics, 58(5): 453-470.

Gai, P., and S. Kapadia. 2010. "Contagion in Financial Networks." Proceedings of the Royal Society A, 466: 2401-2423.

Gale, D., and X. Vives. 2002. "Dollarization, Bailouts, and the Stability of the Banking System." Quarterly Journal of Economics, 117(2): 467-502.

Glasserman, P., and H.P. Young. 2015. "How Likely is Contagion in Financial Networks?" Journal of Banking and Finance, 50: 383-399.

Glasserman, P., and H.P. Young. 2016. "Contagion in Financial Networks." Journal of Economic Literature, 54(3): 779-831. 


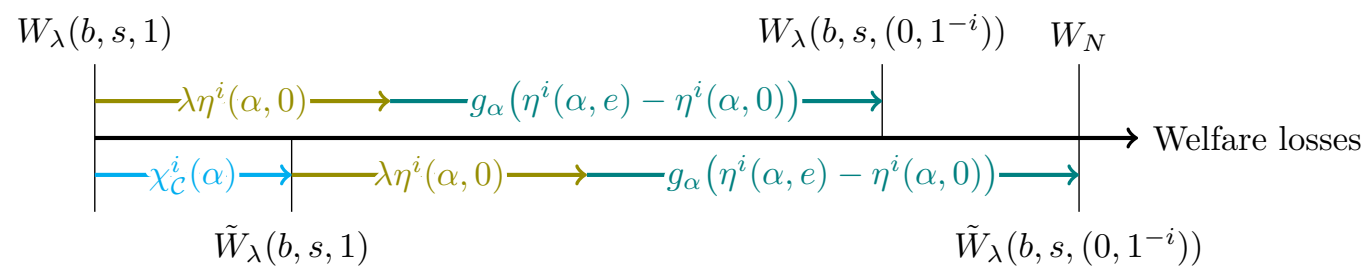

Figure 6: Consider an individually incentive-compatible bail-in $(b, s)$ that induces asset price $\alpha$, in which bank $i$ contributes the largest incentive-compatible amount $\eta^{i}(\alpha, e)$. A rejection by bank $i$ has an impact of $\lambda \eta^{i}(\alpha, 0)+$ $g_{\alpha}\left(\eta^{i}(\alpha, e)-\eta^{i}(\alpha, 0)\right)$ on welfare (top). If the regulator burns $\chi_{\mathcal{C}}^{i}(\alpha)=\left(W_{N}-W_{\lambda}\left(b, s,\left(0,1^{-i}\right)\right)\right)^{+}$units of welfare in a proposal that is otherwise identical, he becomes indifferent between the residual bail-in without bank $i$ and the default cascade (bottom). Thus, bank $i$ can no longer free-ride on the contributions of the remaining banks.

Gorton, G. 2010. "Are Naked Credit Default Swaps too Revealing?" Investment Dealers' Digest, 76: 21-22.

Greenspan, A. 1998. "Testimony by the Chairman of the Federal Reserve Board before the Committee on Banking and Financial Services of the US House of Representatives."

Greenwald, B., and J.E. Stiglitz. 2003. Towards a New Paradigm in Monetary Economics. Cambridge University Press.

Hurley, N., and S. Rickard. 2009. "Comparing Measures of Sparsity." IEEE Transactions on Information Theory, , (10): 4723-4741.

Keister, T. 2016. "Bailouts and Financial Fragility." Review of Economic Studies, 83(2): 704-736.

May, R.A., S.A. Levin, and G. Sugihara. 2008. "Complex Systems: Ecology for Bankers." Nature, 451: 893-895.

Nier, E., J. Yang, T. Yorulmazer, and A. Alentorn. 2007. "Network Models and Financial Stability." Journal of Economic Dynamics and Control, 31(6): 2033-2066.

Rogers, L.C.G., and L.A.M. Veraart. 2013. "Failure and Rescue in an Interbank Network." Management Science, 59(4): 882-898.

Stiglitz, J.E. 2002. Globalization and its Discontents. W.W. Norton.

Upper, C., and A. Worms. 2004. "Estimating Bilateral Exposures in the German Interbank Market: Is There a Danger of Contagion?" European Economic Review, 48: 827-849.

\section{A Welfare Burning}

In this appendix, we define the minimal amount $\chi_{\mathcal{C}}(\alpha)$ of welfare burning needed to eliminate freeriding incentives from an individually incentive-compatible bail-in with contributing banks in $\mathcal{C}$.

Lemma A.1. Let $z(\alpha):=\alpha \ln (\alpha)$ and let $z^{-1}$ be its inverse on the interval $\left[\frac{1}{\mathrm{e}}, 1\right]$. Define the function $g_{\alpha}(x):=g\left(z^{-1}(z(\alpha)+\gamma x)\right)-g(\alpha)$, which is invertible for $\alpha \geq \alpha_{\text {ind }}$. For a set of banks $\mathcal{C}$, let $\chi_{\mathcal{C}}^{i}(\alpha):=\left(W_{N}-W_{P}+g\left(\alpha_{P}\right)-g(\alpha)+\lambda \sum_{j \in \mathcal{C} \backslash\{i\}} \eta^{j}(\alpha, 0)-g_{\alpha}\left(\eta^{i}(\alpha, e)-\eta^{i}(\alpha, 0)\right)\right)^{+}$for any bank $i \in \mathcal{C}$. Moreover, let $\hat{\chi}_{\mathcal{C}}(\alpha)$ denote the unique non-negative solution $\chi$ to

$$
-\frac{\alpha \ln (\alpha)}{\gamma}=\sum_{i \in \mathcal{C}} g_{\alpha}^{-1}\left(\left(W_{N}-W_{P}+g\left(\alpha_{P}\right)-\lambda \sum_{j \in \mathcal{C} \backslash\{i\}} \eta^{i}(\alpha, 0)-\chi\right)^{+}\right)
$$

if it exists and let $\hat{\chi}_{\mathcal{C}}(\alpha)=0$ otherwise. Define $\chi_{\mathcal{C}}(\alpha):=\hat{\chi}_{\mathcal{C}}(\alpha) \vee \max _{i \in \mathcal{C}} \chi_{\mathcal{C}}^{i}(\alpha)$. Consider a bail-in $(b, s)$ with accepting equilibrium $1=(1, \ldots, 1)$. Let $\mathcal{C}=\left\{i \mid b^{i}>0\right\}$ and $\alpha=\bar{\alpha}(b, s, 1)$. Then

$$
\lambda \sum_{i \notin \mathcal{C}}\left(s^{i}-s_{0}^{i}\right)^{+}+\lambda \sum_{i \in \mathcal{C}}\left(\eta^{i}(\alpha, 0)-b^{i}\right)^{+} \geq \chi_{\mathcal{C}}(\alpha)
$$




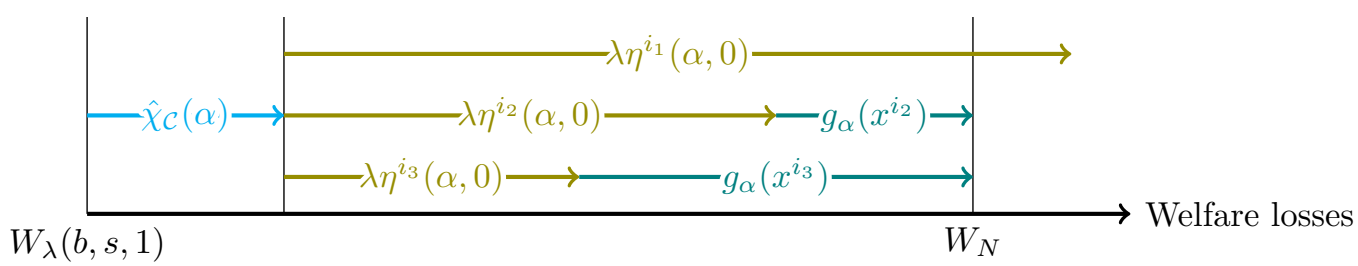

Figure 7: The construction of $\hat{\chi}_{\mathcal{C}}(\alpha)$ is illustrated for $\mathcal{C}=\left\{i_{1}, i_{2}, i_{3}\right\}$. It is the minimal amount of welfare burnt such that the welfare impact $\lambda \eta^{i}(\alpha, 0)+g_{\alpha}\left(x^{i}\right)$ caused by the rejection of any bank $i \in \mathcal{C}$ exceeds $W_{N}-W_{\lambda}(b, s, 1)-\hat{\chi}_{\mathcal{C}}(\alpha)$, subject to the constraint $\sum_{i \in \mathcal{C}} x^{i} \leq-\alpha \ln (\alpha) / \gamma$, which guarantees that the total amount liquidated does not depress the asset price below $\alpha$. Note that $x^{i_{3}}=0$ because the welfare impact of $i_{3}$ 's rejection is large enough to deter freeriding without asset liquidation. If $\hat{\chi}_{\mathcal{C}}(\alpha)>0$, then the constraint binds, that is, $x^{i_{2}}+x^{i_{3}}=-\alpha \ln (\alpha) / \gamma$. Otherwise, it would be possible to increase the welfare impact by banks $i_{1}$ and $i_{2}$ and decrease the amount of welfare burnt.

For the no-free-riding incentives to hold, the rejection by any bank must have an impact on welfare in excess of $W_{N}-W_{\lambda}(b, s, 1)$. It follows from (14) that the impact on welfare has two components: contributions up to $\eta(\alpha, 0)$ affect welfare directly by an amount $\lambda \eta(\alpha, 0)$, whereas contributions that exceed $\eta(\alpha, 0)$ by an amount $x$ require asset liquidation and affect welfare through the trade-off $g_{\alpha}(x)$. A contribution of size $\eta^{i}(\alpha, 0)+x^{i}$ by bank $i$ thus has a total impact on welfare of $\lambda \eta^{i}(\alpha, 0)+g_{\alpha}\left(x^{i}\right)$. Among individually incentive-compatible contributions, the welfare impact by bank $i$ is maximized when $i$ contributes $\eta^{i}(\alpha, e)$. If the maximal welfare impact is smaller than $W_{N}-W_{\lambda}(b, s, 1)$, the remaining value $\chi_{\mathcal{C}}^{i}(\alpha)=W_{N}-W_{\lambda}(b, s, 1)-\lambda \eta^{i}(\alpha, 0)-g_{\alpha}\left(\eta^{i}(\alpha, e)-\eta^{i}(\alpha, 0)\right)$ has to be burnt; see Figure 6 for an illustration. Burning an amount equal to $\max _{i \in \mathcal{C}} \chi_{\mathcal{C}}^{i}(\alpha)$ thus deters free-riding by all banks when each bank liquidates the maximal incentive-compatible amount. However, asking each bank to contribute $\eta^{i}(\alpha, e)$ may require asset liquidation in excess of $-\ln (\alpha) / \gamma$, which would depress the asset price below $\alpha$ by (1). In that case, the regulator has to ask for lower contributions from banks that require aggregate liquidation of only $-\ln (\alpha) / \gamma$ and burn additional welfare to balance the reduced welfare impact of the contributing banks. The lowest amount of welfare burnt in that case is $\hat{\chi}_{\mathcal{C}}(\alpha)$; see Figure 7 for an illustration.

\section{B Proofs of Results in Sections 2 and 3}

\section{B.1 Existence and Monotonicity of Clearing Equilibria}

This appendix provides the proofs of Section 2, asserting existence and monotonicity of clearing equilibria, together with Pareto dominance of the greatest clearing equilibrium. We begin with the following auxiliary result, which provides us with an alternative expression for welfare losses.

Lemma B.1. For any clearing equilibrium $(p, \ell, \alpha)$, the following identity holds

$$
W_{\lambda}(p, \alpha)=\sum_{i=1}^{n}\left(c^{i}+e^{i}-w^{i}-V^{i}(p, \alpha)\right)+\sum_{i \in \mathcal{D}(p, \ell, \alpha)}(1+\lambda) \delta^{i}(p, \alpha) .
$$

Proof. Observe first that $\pi$ is column-stochastic by construction, i.e., the columns sum up to 1 . Thus, $\sum_{i}(\pi x)^{i}=\sum_{i} x^{i}$ for any vector $x$. Summing (4) for prices $(p, \alpha)$, we obtain

$$
\sum_{i=1}^{n} V^{i}(p, \alpha)=\sum_{i=1}^{n}\left(c^{i}+\alpha e^{i}-w^{i}\right)-\sum_{i \in \mathcal{D}(p, \ell, \alpha)}\left(c^{i}-w^{i}+\alpha e^{i}+(\pi p)^{i}-p^{i}\right) .
$$


It follows that

$$
\sum_{i=1}^{n}\left(c^{i}+e^{i}-w^{i}-V^{i}(p, \alpha)\right)=\sum_{i=1}^{n}(1-\alpha) e^{i}+\sum_{i \in \mathcal{D}(p, \ell, \alpha)}\left(c^{i}-w^{i}+\alpha e^{i}+(\pi p)^{i}-p^{i}\right) .
$$

The definition of a clearing payment vector in (3) implies that $p^{i}+w^{i}-\delta^{i}=\beta\left(c^{i}+\alpha e^{i}+(\pi p)^{i}\right)$ for any $i \in \mathcal{D}(p, \ell, \alpha)$. Therefore, we obtain

$$
c^{i}+\alpha e^{i}+(\pi p)^{i}-\left(p^{i}+w^{i}-\delta^{i}\right)=(1-\beta)\left(c^{i}+\alpha e^{i}+(\pi p)^{i}\right)
$$

for any $i \in \mathcal{D}(p, \ell, \alpha)$. Equation (28) now follows from (29) and (30).

Since the equity value of any bank is monotonically increasing in prices, a direct consequence of Lemma B.1 is the fact that welfare losses are monotonically decreasing in prices. For the sake of reference, we state this property as a lemma.

Lemma B.2. Any bank i's value of equity $V^{i}(p, \alpha)$ is non-decreasing and welfare losses $W_{\lambda}(p, \alpha)$ are non-increasing in $\alpha$ and $p^{j}$ for any $j$.

Proof. Bank $i$ 's value in (4) is equal to $V^{i}(p, \alpha)=(\pi p+c+\alpha e-w-L)^{i} 1_{\left\{p^{i}=L^{i}\right\}}$, which is nondecreasing in $(p, \alpha)$. This also implies that the first term of welfare losses in $(28)$ is non-increasing in $(p, \alpha)$. The second term in $(28)$ is non-increasing in $(p, \alpha)$ by definition of $\delta(p, \alpha)$ in $(5)$.

Proof of Lemma 2.2. Let $\mathcal{L}:=\left[0, e^{1}\right] \times \cdots \times\left[0, e^{n}\right]$ denote the set of possible liquidation decisions by the banks. Fix a vector $p$ of interbank repayments and define the operator $\Phi_{p}: \mathcal{L} \rightarrow \mathcal{L}$ by setting $\Phi_{p}^{i}(x):=\ell^{i}(\alpha(x), p)$ for $i=1, \ldots, n$, where $\alpha(x)$ and $\ell(\alpha, p)$ are defined in (1) and (2). By construction, a pair $(\alpha, \ell)$ is a solution to (1) and (2) if and only if it is of the form $(\alpha(x), x)$ for a fixed point $x$ of $\Phi_{p}$. Since both $\alpha$ and $\ell^{i}$ are non-increasing, $\Phi_{p}^{i}$ is non-decreasing. Therefore, Tarski's fixed-point theorem implies that the set of $\Phi_{p}$ 's fixed points forms a complete lattice. In particular, there exists a fixed point $\underline{x}$ such that $\underline{x}^{i} \leq x^{i}$ for each $i$ and any other fixed point $x$ of $\Phi_{p}$. Let $\ell_{p}=\underline{x}$ and $\alpha_{p}=\alpha\left(\ell_{p}\right)$. By construction, $\left(\alpha_{p}, \ell_{p}\right)$ satisfies (1) and (2) and for any other solution $(\tilde{\alpha}, \tilde{\ell})$, we have $\tilde{\alpha}=\exp \left(-\gamma \sum_{i=1}^{n} \tilde{\ell}^{i}\right) \leq \exp \left(-\gamma \sum_{i=1}^{n} \ell_{p}^{i}\right)=\alpha_{p}$.

Before proving existence of clearing equilibria, we show the following comparison result for fixed points that will be used many times throughout our analysis.

Lemma B.3. Let $f$ and $g$ be two non-decreasing functions mapping a compact set $\mathcal{X}$ into itself. Let $\bar{x}_{f}\left(\underline{x}_{f}\right)$ and $\bar{x}_{g}\left(\underline{x}_{g}\right)$ denote the largest (smallest) fixed points of $f$ and $g$, respectively, that exist by Tarski's fixed point theorem. If $f\left(\bar{x}_{g}\right) \geq g\left(\bar{x}_{g}\right)$, then $\bar{x}_{f} \geq \bar{x}_{g}$. If $f\left(\underline{x}_{g}\right) \leq g\left(\underline{x}_{g}\right)$, then $\underline{x}_{f} \geq \underline{x}_{g}$.

Proof. Let $\bar{x}_{n}:=f^{(n)}\left(\bar{x}_{g}\right)$ define the $n$-fold application of $f$ to $\bar{x}_{g}$. Since $f\left(\bar{x}_{g}\right) \geq g\left(\bar{x}_{g}\right)=\bar{x}_{g}$ and $f$ is non-decreasing, it follows that $\left(\bar{x}_{n}\right)_{n \geq 1}$ is non-decreasing in each component. Because of compactness, $\left(\bar{x}_{n}\right)_{n \geq 1}$ converges to some fixed point $\bar{x}_{\infty}$ of $f$. Therefore, $\bar{x}_{g} \leq \bar{x}_{\infty} \leq \bar{x}_{f}$ because $\bar{x}_{f}$ is the largest fixed point of $f$. The analogous argument shows $\underline{x}_{g} \geq \lim _{n \rightarrow \infty} f^{(n)}\left(\underline{x}_{g}\right) \geq \underline{x}_{f}$. 
We are now ready to prove Lemma 2.1.

Proof of Lemma 2.1. Let $\mathcal{P}:=\left[0, L^{1}\right] \times \cdots \times\left[0, L^{n}\right]$ denote the set of possible clearing payment vectors. For fixed $p$, let $\left(\alpha_{p}, \ell_{p}\right)$ be the solutions to (1) and (2) of Lemma 2.2. Define the operator $\Phi: \mathcal{P} \rightarrow \mathcal{P}$ by setting

$$
\Phi^{i}(p):= \begin{cases}L^{i} & \text { if } c^{i}+\alpha_{p} e^{i}+(\pi p)^{i} \geq L^{i}+w^{i} \\ \left(\beta\left(c+\alpha_{p} e+\pi p\right)^{i}-w^{i}\right)^{+} & \text {otherwise. }\end{cases}
$$

The definition of $\ell_{p}$ in (2) implies that $c^{i}+\alpha_{p} e^{i}+(\pi p)^{i} \geq L^{i}+w^{i}$ if and only if $c^{i}+\alpha_{p} \ell_{p}^{i}+(\pi p)^{i} \geq$ $L^{i}+w^{i}$. Therefore, the definition of a clearing equilibrium implies that a clearing payment vector $p$ is a fixed point of $\Phi$. We proceed to show that $\Phi$ is monotone. It follows directly from Eq. (2) that, for any $i, \ell^{i}(\alpha, p)$ is non-increasing in $p^{j}$ for any $j$. Using the definition of $\Phi_{p}$ given in the proof of Lemma 2.2, we deduce that $\Phi_{\left(\tilde{p}^{j}, p^{-j}\right)}^{i}\left(\ell_{p}\right) \leq \Phi_{p}^{i}\left(\ell_{p}\right)$ for any $\tilde{p}^{j}>p^{j}$. Therefore, Lemma B.3 shows that $\ell_{\left(\tilde{p}^{j}, p^{-j}\right)}^{i} \leq \ell_{p}^{i}$ and hence $\alpha_{\left(\tilde{p}^{j}, p^{-j}\right)} \geq \alpha_{p}$. This shows that $p \mapsto \alpha_{p}$ is non-decreasing, hence so is $\Phi^{i}$. Tarski's fixed point thus implies the existence of fixed points $\underline{p} \leq \bar{p}$ with $\underline{p} \leq p \leq \bar{p}$ for any fixed point $p$. The first statement now follows from the monotonicity of the maps $p \mapsto \alpha_{p}$ and $p \mapsto \ell_{p}$ by setting $\underline{\alpha}=\alpha_{\underline{p}}, \bar{\alpha}=\alpha_{\bar{p}}, \underline{\ell}=\ell_{\underline{p}}$, and $\bar{\ell}=\ell_{\bar{p}}$. Monotonicity of the banks' equity value and welfare losses now follows from Lemma B.2

\section{B.2 Bailouts}

This appendix shows that welfare-maximizing bailouts are of the form given in Lemma 3.2 . We begin this appendix with the following auxiliary lemma.

Lemma B.4. The function $g(\alpha)=\alpha\left(\frac{\lambda}{\gamma} \ln (\alpha)-\sum_{i=1}^{n} e^{i}\right)$ is strictly convex, attaining its global minimum at $\alpha_{\text {ind }}$ defined in 10 .

Proof. Since $g$ is differentiable on $(0, \infty)$, the product rule yields

$$
g^{\prime}(\alpha)=\frac{\lambda}{\gamma}(1+\ln (\alpha))-\sum_{i=1}^{n} e^{i}, \quad g^{\prime \prime}(\alpha)=\frac{\lambda}{\gamma \alpha}>0
$$

proving that $g$ is strictly convex. Since $g^{\prime}\left(\alpha_{\text {ind }}\right)=0, \alpha_{\text {ind }}$ is the global minimizer of $g$.

Proof of Lemma 3.1. Let $s$ denote a vector of subsidies of a complete bailout with $s^{i} \leq s_{0}^{i}$ for every bank $i$. Since every bank is rescued when subsidies $s$ are awarded, the definition of $s_{0}$ implies that $\ell^{i}=\frac{1}{\bar{\alpha}(s)}\left(s_{0}^{i}-s^{i}\right)$ for any bank $i$. It follows from (1) that

$$
\bar{\alpha}(s)=\exp \left(-\frac{\gamma}{\bar{\alpha}(s)} \sum_{i=1}^{n}\left(s_{0}^{i}-s^{i}\right)\right)
$$

Solving (32) for $\sum_{i=1}^{n} s^{i}$ and substituting into (7) shows (9). 
Proof of Lemma 3.2. Let $s$ denote a vector of subsidies that maximize welfare in a complete bailout. Because a minimal subsidy of $s_{L}$ is needed to support the clearing payment vector $L$, it follows that $s^{i} \geq s_{L}^{i}$ for every bank $i$. Since any subsidies beyond $s_{0}$ have infinitesimal welfare impact $-\lambda$, it follows that $s^{i} \leq s_{0}^{i}$ for every bank $i$. Therefore, $\ell^{i}=\frac{1}{\alpha_{P}}\left(s_{0}^{i}-s^{i}\right)$ for any bank $i$, which implies

$$
\alpha_{P}=\exp \left(-\frac{\gamma}{\alpha_{P}} \sum_{i=1}^{n}\left(s_{0}^{i}-s^{i}\right)\right)
$$

Lemma 3.1 establishes that welfare in the complete bailout depends on the awarded subsidies only through $g\left(\alpha_{P}\right)$. Since $g$ is differentiable, it follows from B.4 that $\alpha_{P}$ is either a boundary point or it is equal to $\alpha_{\text {ind }}$. Since (33) is monotonic in the awarded subsidies, boundary points are attained at $\alpha_{L}$ and 1 when the subsidies are equal to $s_{L}$ and $s_{0}$, respectively. It follows from monotonicity in (33) that $\alpha_{P}=\max \left(\min \left(\alpha_{\text {ind }}, 1\right), \alpha_{L}\right)$. Inverting (33) for $\sum_{i=1}^{n} s^{i}$ yields (11).

\section{B.3 Incentives and Bail-In Selection}

In this appendix we provide the proofs of the results in Section 3.4, characterizing accepting equilibria and how the regulator can select among multiple accepting equilibria. We begin with the following auxiliary result, formalizing that a bank is better off rejecting a bail-in proposal if its participation is not needed for the regulator to proceed with the bail-in. It will be convenient to denote by $V^{i}(b, s, a)=V^{i}(\bar{p}(b, s, a), \bar{\alpha}(b, s, a))$ bank $i$ 's value of equity in the bail-in $(b, s, a)$. Similarly, let $V^{i}(s)=V^{i}(\bar{p}(s), \bar{\alpha}(s))$ denote bank $i$ 's value of equity in the bailout with subsidies $s$.

Lemma B.5. Fix a feasible bail-in proposal $(b, s)$ with $b^{i}>0$ for some bank $i$. For any response $a^{-i}$ by $i$ 's competitors, we have $V^{j}\left(b, s,\left(0, a^{-i}\right)\right) \geq V^{j}\left(b, s,\left(1, a^{-i}\right)\right)$ for any bank $j$. Moreover, the inequality is strict if $j=i$.

Proof. The two financial systems resulting from $\left(b, s,\left(0, a^{-i}\right)\right)$ and $\left(b, s,\left(1, a^{-i}\right)\right)$ are identical up to the financial commitments by bank $i$, which are larger by $b^{i}$ in the latter system. The result thus follows from Statement 3 in Lemma F.3 of the online appendix and monotonicity in Lemma B.2.

Proof of Lemma 3.5. Fix a feasible proposal $(b, s)$ with accepting equilibrium response $a$. We first show necessity of the stated conditions. To this end, fix a bank $i$ with $b^{i}>0$ and suppose towards a contradiction that $a^{i}=1$ but at least one of the two conditions is violated. Consider first the case where Condition 1 is violated. Then the regulator proceeds with a bail-in even if bank $i$ rejects the proposal. Therefore, the resulting subsidies are the same under $a$ and $\left(0, a^{-i}\right)$. It follows from Lemma B.5 that bank $i$ is strictly better off under $\left(0, a^{-i}\right)$, contradicting the assumption that $a$ is an equilibrium. Consider now the case where Condition 2 is violated. Because $(b, s)$ is feasible, bank $i$ can afford to pay its liabilities $L^{i}+w^{i}$ and the bail-in contribution $b^{i}$. Together with the negation of Condition 2, we obtain

$$
L^{i}+w^{i} \leq c^{i}+s^{i}-b^{i}+\alpha\left(b, s,\left(1, a^{-i}\right)\right) e^{i}+\sum_{j=1}^{n} \pi^{i j} L^{j}<c^{i}+\alpha_{N} e^{i}+\sum_{j=1}^{n} \pi^{i j} p_{N}^{j}
$$


This shows that bank $i$ is solvent in the default cascade. Therefore, subtracting $L^{i}+w^{i}$ in (34) implies that $V_{N}^{i}>V^{i}\left(b, s,\left(1, a^{-i}\right)\right)$, which is again a contradiction.

For sufficiency, Condition 1 implies that the regulator will react to a rejection by any bank by letting a default cascade occur. By Condition 2, such an outcome makes bank $i$ worse off. It is thus optimal for bank $i$ to accept the proposal.

Proof of Lemma 3.6. Fix a bail-in $(b, s)$ with an accepting equilibrium $a_{k}$. Let $\mathcal{B}=\left\{i \mid b^{i} 1_{\left\{a_{k}^{i}=1\right\}}\right\}$ denote the set of banks making a positive contribution in $\left(b, s, a_{k}\right)$. Define a bail-in $(\tilde{b}, \tilde{s})$ by setting $\tilde{b}^{i}=b^{i} 1_{\{i \in \mathcal{B}\}}$ and $\tilde{s}^{i}=s^{i}$ for $i=1, \ldots, n$. We proceed to show that $1=(1, \ldots, 1)$ is an accepting equilibrium response to $(\tilde{b}, \tilde{s})$. Note that, by convention, the only available response for any bank $i \in \mathcal{B}^{c}$ is to accept the proposal; see Footnote 15 . For $i \in \mathcal{B}$, note that Lemma 3.5 shows that the stated Conditions 1 and 2 are satisfied in $\left(b, s, a_{k}\right)$ for any $i \in \mathcal{B}$ since $a_{k}$ is an accepting equilibrium. Because each bank makes the same contribution in $(\tilde{b}, \tilde{s}, 1)$ as in $\left(b, s, a_{k}\right)$, it follows that $\bar{\alpha}(\tilde{b}, \tilde{s}, 1)=\alpha\left(b, s, a_{k}\right), W_{\lambda}(b, s, 1)=W_{\lambda}\left(b, s, a_{k}\right)$, as well as $W_{\lambda}\left(b, s,\left(0,1^{-i}\right)\right)=$ $W_{\lambda}\left(b, s,\left(0, a_{k}^{-i}\right)\right)$ for every $i \in \mathcal{B}$. Therefore, Conditions 1 and 2 of Lemma 3.5 are satisfied also in $(\tilde{b}, \tilde{s})$ for any $i \in \mathcal{B}$. Lemma 3.5 thus implies that 1 is an accepting equilibrium response to $(\tilde{b}, \tilde{s})$. To show uniqueness, observe that the regulator will not proceed with the bail-in if only a proper subset of $\mathcal{B}$ accepts the proposal due to Condition 1 of Lemma 3.5.

Proof of Lemma 3.7. Fix a complete bail-in proposal $(b, s)$ with accepting equilibrium $a$. It follows from Lemma 3.5 that the value of any bank in $\mathcal{A}(b)=\left\{i \mid b^{i}>0\right\}$ is at least as high in $(b, s, a)$ as in a rejecting equilibrium as otherwise bank $i$ would have not accepted the proposal. By definition of an accepting equilibrium, welfare losses are at most as high as in a rejecting equilibrium, because otherwise the regulator would not have proceeded with the bail-in. This shows that (a) no rejecting equilibrium can subgame Pareto dominate $a$ and (b) a rejecting equilibrium is subgame Pareto efficient only if it is equivalent to $(b, s, a)$. Since there are only two possible outcomes in a rejecting equilibrium (public bailout and no rescue), the complete bail-in proposal $(b, s)$ has to coincide with the complete bailout as, by definition, it rescues every bank in the system.

Let $\tilde{\mathcal{A}}$ denote the set of accepting equilibria that minimize welfare losses. It remains to show that at least one of them is subgame Pareto efficient. Let $a \in \mathcal{A}$ denote the accepting equilibrium leading to the highest asset price among accepting equilibria in $\mathcal{A}$. Suppose towards a contradiction that there exists a continuation equilibrium $\tilde{a}$ that Pareto dominates $a$. This necessitates $W_{\lambda}(b, s, \tilde{a}) \leq W_{\lambda}(b, s, a)$, which implies that $\tilde{a}$ is an accepting equilibrium in $\mathcal{A}$. Let $\mathcal{C}$ and $\tilde{\mathcal{C}}$ denote the set of banks which accept the proposal in $a$ and $\tilde{a}$, respectively. By Condition 1 of Lemma 3.5 . the regulator rejects the bail-in if only a strict subset of $\mathcal{C}$ accepts the proposal. Since $\tilde{a}$ is an accepting equilibrium, $\tilde{\mathcal{C}}$ is not a subset of $\mathcal{C}$ and hence $\tilde{\mathcal{C}} \backslash \mathcal{C} \neq \emptyset$. By maximality of $a$ in $\mathcal{A}$, we have $\alpha(b, s, \tilde{\alpha}) \leq \alpha(b, s, a)$. Thus, because each bank in $\tilde{\mathcal{C}} \backslash \mathcal{C}$ makes a positive contribution in $\tilde{a}$ but not in $a$, each such bank is is strictly worse off in $\tilde{a}$ than in $a$. This contradicts the assumption that $\tilde{a}$ Pareto dominates $a$, which concludes the proof. 


\section{B.4 Credibility and Existence of Subgame Pareto Efficient Equilibria}

In this appendix we prove Lemma 2.3, which establishes existence of subgame Pareto-efficient continuation equilibria after the proposal of any bail-in. In order to present the proofs as succinctly as possible, we invoke Proposition 3.3 to deal with the case when the threat fails to be credible. The proof of Proposition 3.3 does not rely on Lemma 2.3. existence of equilibria when the threat fails to be credible follows directly from the existence of strictly dominant strategies.

Proof of Proposition 3.3. Fix a feasible proposal $(b, s)$ and any response vector $a$. Because the threat fails to be credible, the regulator will never respond with "no intervention". Since any bank $i$ with $b^{i}-s^{i}>0$ is strictly worse off in $(b, s)$ than in a complete rescue without its participation (e.g., bailout or bail-in by residual consortium) by Lemma B.5, rejection is the strictly dominant action for any such bank. This shows that the regulator cannot get any contributions from banks, hence the optimal bailout of Lemma 3.2 is the only possible equilibrium outcome.

Together with the characterization of accepting equilibria in Lemma 3.5. Proposition 3.3 allows us to prove Lemma 2.3 .

Proof of Lemma 2.3. If the threat fails to be credible, Proposition 3.3 establishes that a rejecting equilibrium exists after any proposal $(b, s)$. Suppose, therefore, that the threat is credible. Fix a feasible proposal $(b, s)$ and let 0 denote the vector of unanimous rejections by the banks. The credibility of the threat imposes that $W_{N} \leq W_{P} \leq W_{\lambda}(b, s, 0)$, where we have used the fact that without participation of the banks, the optimal rescue is the public bailout of Lemma 3.2. The regulator thus chooses $r(b, s, 0)=$ "no intervention". If no bank in $\mathcal{A}(b)$ has a profitable unilateral deviation to the vector of unanimous rejections, then that response is a rejecting equilibrium. Suppose, therefore, that there exists a bank $i \in \mathcal{A}(b)$ with a profitable unilateral deviation and let $a_{i}$ denote the corresponding action profile. We will show that $a_{i}$ is an equilibrium response.

For $a_{i}$ to be a profitable deviation for bank $i$, two conditions must hold. First, it is necessary that $r\left(b, s, a_{i}\right)=$ "bail-in" as otherwise, bank $i$ 's value of equity would be equal to $V_{N}^{i}$ both when $i$ accepts and when $i$ rejects the proposal. Second, we must have that $V^{i}\left(b, s, a_{i}\right)>V_{N}^{i}$. This condition is equivalent to $b^{i}-s^{i}<\sum_{j=1}^{n} \pi^{i j}\left(L^{j}-p_{N}^{j}\right)+\left(\alpha\left(b, s, a_{i}\right)-\alpha_{N}\right) e^{i}$, i.e., Condition 2 of Lemma 3.5 is satisfied for bank $i$. Since, by construction, $\left(0, a_{i}^{-i}\right)$ is the vector of unanimous rejections, it follows that $W_{\lambda}\left(b, s,\left(0, a_{i}^{-i}\right)\right)=W_{\lambda}(b, s, 0) \geq W_{N}$, i.e., Condition 1 of Lemma 3.5 is satisfied for bank $i$ as well.

It remains to show that for any bank $j \neq i$, either the conditions of Lemma 3.5 is violated. For the regulator to choose "bail-in" in reaction to $a_{i}$, it is necessary that $W_{\lambda}\left(b, s, a_{i}\right)<W_{N}$. Since $\left(0, a_{i}^{-j}\right)=a_{i}$ for any bank $j \neq i$, it follows that $W_{\lambda}\left(b, s,\left(0, a_{i}^{-j}\right)\right)=W_{\lambda}\left(b, s, a_{i}\right)<W_{N}$ for any such bank. Therefore, Condition 1 of Lemma 3.5 is violated for any bank in $\mathcal{A}(b) \backslash\{i\}$. An application of Lemma 3.5 thus shows that $a_{i}$ is an accepting equilibrium.

We conclude this appendix by proving that the regulator's threat is credible if and only if the amplification of the shock through the network is below the threshold given in Lemma 3.4 . 
Proof of Lemma 3.4. Observe first that $\left(L^{i}+w^{i}-c^{i}-e^{i}-(\pi L)^{i}\right)^{-}=\left(s_{0}^{i}-e^{i}\right)^{+}=s_{0}^{i}-e^{i}-\left(e^{i}-s_{0}^{i}\right)^{+}$. It follows from (28) and the definitions of $S_{0}$ and $S_{N}$ that welfare in the default cascade can be written as

$$
W_{N}=S_{N}-S_{0}+\sum_{i=1}^{n}\left(e^{i}+\left(e^{i}-s_{0}^{i}\right)^{+}\right)+(1+\lambda) \sum_{i \in \mathcal{D}\left(p_{N}, \ell_{N}, \alpha_{N}\right)} \delta^{i}\left(p_{N}, \alpha_{N}\right) .
$$

Lemma 3.2 shows that $W_{P}=\sum_{i=1}^{n} e^{i}+\lambda S_{0}+g\left(\alpha_{P}\right)+\lambda \sum_{i \in \mathcal{D}\left(p_{N}, \ell_{N}, \alpha_{N}\right)} \delta^{i}\left(p_{N}, \alpha_{N}\right)$. Solving the inequality $W_{N}-W_{P} \leq 0$ for $S_{N}-S_{0}$ using the above expressions for $W_{N}$ and $W_{P}$, we obtain (12).

\section{Proof of Theorem 3.10}

We begin this section by showing that without loss of generality, we can restrict our attention to bail-ins, where each bank either makes a contribution or receives a subsidy. Moreover, because the regulator can anticipate the banks' responses, we may also restrict our attention to bail-ins that allow response vector 1 in equilibrium.

Lemma C.1. For any proposed bail-in $(b, s)$ with accepting equilibrium response a, there exists a proposal $(\tilde{b}, \tilde{s})$ with accepting continuation equilibrium $\tilde{a}$ such that $\tilde{b}^{i} \tilde{s}^{i}=0$ and $\tilde{a}^{i}=1$ for every bank $i$, and $W_{\lambda}(\tilde{b}, \tilde{s}, \tilde{a})=W_{\lambda}(b, s, a)$.

Proof. Fix a bail-in proposal $(b, s)$ with accepting equilibrium response $a$. The existence of an accepting equilibrium $a$ implies via Lemma 3.7 that either $(b, s)$ is the public bailout of Lemma 3.2 or that the threat is credible. In the former case, the statement holds trivially, hence suppose that the threat is credible. Denote by $\mathcal{C}=\left\{b^{i}-s^{i}>0, a^{i}=1\right\}$ the set of banks which make a positive net contribution. Define the proposal $(\tilde{b}, \tilde{s})$ by setting $\tilde{b}^{i}=\left(b^{i} 1_{\left\{a^{i}=1\right\}}-s^{i}\right)^{+}$and $\tilde{s}^{i}=\left(s^{i}-b^{i} 1_{\left\{a^{i}=1\right\}}\right)^{+}$, and let $\tilde{a}=(1, \ldots, 1)$ be the response vector of unanimous acceptance. It follows straight from the construction of $(\tilde{b}, \tilde{s})$ that $\tilde{b}^{i} 1_{\left\{\tilde{a}^{i}=1\right\}}-\tilde{s}^{i}=b^{i} 1_{\left\{a^{i}=1\right\}}-s^{i}$ for any bank $i$ and hence each bank's net contribution remains unchanged. This implies that clearing equilibria coincide both in $(\tilde{b}, \tilde{s}, \tilde{a})$ and $(b, s, a)$ and also in $\left(\tilde{b}, \tilde{s},\left(0, \tilde{a}^{-i}\right)\right)$ and $\left(b, s,\left(0, a^{-i}\right)\right)$ for any $i \in \mathcal{C}$. It follows that

$$
\begin{aligned}
W_{\lambda}(\tilde{b}, \tilde{s}, \tilde{a}) & =W(\bar{p}(\tilde{b}, \tilde{s}, \tilde{a}), \bar{\alpha}(\tilde{b}, \tilde{s}, \tilde{a}))+\lambda \sum_{i=1}^{n}\left(\tilde{s}^{i}-\tilde{b}^{i} 1_{\left\{\tilde{a}^{i}=1\right\}}\right) \\
& =W(\bar{p}(b, s, a), \bar{\alpha}(b, s, a))+\lambda \sum_{i=1}^{n}\left(s^{i}-b^{i} 1_{\left\{a^{i}=1\right\}}\right)=W_{\lambda}(b, s, a)
\end{aligned}
$$

and, in a similar fashion, that $W_{\lambda}\left(\tilde{b}, \tilde{s},\left(0, \tilde{a}^{-i}\right)\right)=W_{\lambda}\left(b, s,\left(0, a^{-i}\right)\right)$ for any $i \in \mathcal{C}$. To conclude that $\tilde{a}$ is an accepting equilibrium, it remains to verify Condition 2 of Lemma 3.5. Since $a$ is an accepting equilibrium for $(b, s)$, Lemma 3.5 implies that

$$
\tilde{b}^{i}-\tilde{s}^{i}=b^{i}-s^{i} \leq \sum_{j=1}^{n} \pi^{i j}\left(\bar{p}^{j}(b, s, a)-p_{N}^{j}\right)+\left(\bar{\alpha}(b, s, a)-\alpha_{N}\right) e^{i}
$$


for every $i \in \mathcal{C}$. Because the clearing equilibria under $(\tilde{b}, \tilde{s}, \tilde{a})$ and $(b, s, a)$ coincide, this shows that Condition 2 of Lemma 3.5 is satisfied in $(\tilde{b}, \tilde{s}, \tilde{a})$. An application of Lemma 3.5 thus shows that $\tilde{a}$ is an accepting equilibrium response to $(\tilde{b}, \tilde{s})$.

Because every bank is rescued in a complete feasible bail-in, the shortfall of each bank $i$ before liquidation is at most $e^{i}$. The liquidated amount by any bank $i$ is thus inversely proportional to the asset price. This imposes a lower bound on the asset price of $\frac{1}{\mathrm{e}}=\exp (-1)$.

Lemma C.2. Let $(b, s)$ be a complete feasible bail-in proposal. In any response a, each bank $i$ liquidates $\ell^{i}(b, s, a)=\frac{1}{\alpha}\left(L^{i}+w^{i}+b^{i} 1_{\left\{a^{i}=1\right\}}-c^{i}-s^{i}-(\pi L)^{i}\right)^{+}$and $\alpha(b, s, a) \geq \frac{1}{\mathrm{e}}$.

Proof. Since $(b, s)$ is a complete rescue, Lemma 2.2 implies that $(\alpha(b, s, a), \ell(b, s, a))$ is the solution to (1) and (2) for $p=L$ with the largest asset price. By feasibility,

$$
L^{i}+w^{i}+b^{i} 1_{\left\{a^{i}=1\right\}}-c^{i}-s^{i}-(\pi L)^{i} \leq \alpha(b, s, 1) e^{i} \leq \alpha(b, s, a) e^{i} .
$$

This shows that $\ell^{i}(b, s, a)$ is indeed of the desired form. Therefore, $\alpha(b, s, a)$ is a fixed point of the function $f_{x, y}$ in Lemma F.2 for $y=0$ and $x=\gamma \sum_{i=1}^{n}\left(L^{i}+w^{i}+b^{i} 1_{\left\{a^{i}=1\right\}}-c^{i}-s^{i}-(\pi L)^{i}\right)^{+}$. Since $\alpha(b, s, a)$ is the largest fixed point of $f$ on $(0,1]$, Lemma F.2 implies that $\alpha(b, s, a) \geq \frac{1}{\mathrm{e}}$.

Due to Lemma C.1. we may restrict attention to bail-in proposals $(b, s)$, in which $b^{i} s^{i}=0$ for every bank $i$. Then, liquidation and welfare losses take a simple form as stated in Lemma 3.8 .

Proof of Lemma 3.8. Fix a bail-in $(b, s)$ with an accepting equilibrium $a$ such that $b^{i} s^{i}=0$ for every bank $i$. Let $\alpha=\alpha(b, s, a)$ for the sake of brevity. Lemma C.2 shows that each bank $i$ liquidates an amount $\ell^{i}(b, s, a)=\frac{1}{\alpha}\left(s_{0}^{i}-b_{0}^{i}+b^{i} 1_{\left\{a^{i}=1\right\}}-s^{i}\right)^{+}$. We begin by showing that this coincides with

$$
\ell^{i}(b, s, a)=\frac{1}{\alpha}\left(s_{0}^{i}-\min \left(s^{i}, s_{0}^{i}\right)+\left(b^{i}-\min \left(b^{i}, b_{0}^{i}\right)\right) 1_{\left\{a^{i}=1\right\}}\right) .
$$

Consider first a bank $i$ with $s_{0}^{i}>0$. This implies that $b_{0}^{i}=0$ by definition. If $s^{i}>0$, then $b^{i}=0$ by assumption and hence $\ell^{i}(b, s, a)=\frac{1}{\alpha}\left(s_{0}^{i}-s^{i}\right)^{+}=\frac{1}{\alpha}\left(s^{i}-\min \left(s^{i}, s_{0}^{i}\right)\right)$. This coincides with (35). If $s^{i}=0$ instead, then $\ell^{i}(b, s, a)=\frac{1}{\alpha}\left(s_{0}^{i}+b^{i} 1_{\left\{a^{i}=1\right\}}\right)$ also coincides with (35). Next, consider a bank $i$ with $b_{0}^{i}>0$. For such a bank, $s_{0}^{i}=0$ by definition. If $s^{i}>0$, then $b^{i}=0$ by assumption and hence $\ell^{i}(b, s, a)=0$, which coincides with $(35)$. If $s^{i}=0$, then

$$
\ell^{i}(b, s, a)=\left(b^{i} 1_{\left\{a^{i}=1\right\}}-b_{0}^{i}\right)^{+}=\left(b^{i}-b_{0}^{i}\right)^{+} 1_{\left\{a^{i}=1\right\}}=\left(b^{i}-\min \left(b^{i}, b_{0}^{i}\right)\right) 1_{\left\{a^{i}=1\right\}},
$$

showing that (35) also holds in this case. It follows from (1), (35), and the elementary identity $\min \left(s^{i}, s_{0}^{i}\right)=s^{i}-\left(s^{i}-s_{0}^{i}\right)^{+}$that

$$
\sum_{i=1}^{n}\left(s^{i}-b^{i} 1_{\left\{a^{i}=1\right\}}\right)=\sum_{i=1}^{n}\left(s_{0}^{i}+\left(s^{i}-s_{0}^{i}\right)^{+}-\min \left(b^{i}, b_{0}^{i}\right) 1_{\left\{a^{i}=1\right\}}\right)+\frac{\alpha \ln (\alpha)}{\gamma} .
$$

The result now follows from (7), Lemma 3.2, and the specific form of $g$. 
Lemma 3.9 states that among all individually incentive-compatible bail-ins with contributing banks in $\mathcal{C}$ that induce asset price $\alpha$, welfare is maximized for bail-ins with contributions $\eta^{i}(\alpha, \ell)$ by banks $i \in \mathcal{C}$ for any vector $\ell$ of asset liquidation that induces asset price $\alpha$.

Proof of Lemma 3.9. Fix a complete feasible bail-in proposal $(b, s)$ with accepting equilibrium $a$. By Lemma C.1 we may assume without loss of generality that $b^{i} s^{i}=0$ and $a^{i}=1$ for each bank $i$. For the sake of brevity, denote $\alpha=\alpha(b, s, 1)$. Since $(b, s)$ is a complete rescue, it follows that $s \geq s(\alpha, e)$. Condition 2 of Lemma 3.5 and feasibility imply that $b^{i} \leq \eta^{i}(\alpha, e)$ for each $i \in \mathcal{C}$.

Define the bail-in proposal $(\tilde{b}, \tilde{s})$ by setting $\tilde{b}^{i}=\max \left(b^{i}, \eta^{i}(\alpha, 0)\right)$ for each bank $i \in \mathcal{C}$ and $\tilde{s}^{i}=\min \left(s^{i}, s_{0}^{i}\right)$ for each bank $i \notin \mathcal{C}$. Because subsidies beyond $s_{0}$ and contributions below $\eta^{i}(\alpha, 0)$ do not prevent or require any liquidation, it follows that $\alpha(\tilde{b}, \tilde{s}, 1)=\alpha$. Therefore, Lemma 3.8 implies that subsidies beyond $s_{0}$ and contributions below $\eta^{i}(\alpha, 0)$ are welfare decreasing, i.e., $W_{\lambda}(b, s, 1) \geq W_{\lambda}(\tilde{b}, \tilde{s}, 1)$. Applying Lemma 3.8 for the proposal $(\tilde{b}, \tilde{s})$ thus yields

$$
W_{\lambda}(b, s, 1) \geq W_{\lambda}(\tilde{b}, \tilde{s}, 1)=W_{P}-g\left(\alpha_{P}\right)+g(\alpha)-\lambda \sum_{i \in \mathcal{C}} \eta^{i}(\alpha, 0)
$$

where we have used that $\min \left(\tilde{b}^{i}, b_{0}^{i}\right)=\eta^{i}(\alpha, 0)$. This shows the first statement. The final statement follows by observing that the inequality in (36) holds with equality precisely if $\eta^{i}(\alpha, 0) \leq b^{i}$ for each bank $i \in \mathcal{C}$ and $s^{i} \leq s_{0}^{i}$ for each bank $i \notin \mathcal{C}$.

Next, we show that $\chi_{\mathcal{C}}(\alpha)$ in Lemma A.1 is well-defined. Moreover, any incentive-compatible bail-in with contributing banks in $\mathcal{C}$ that induces asset price $\alpha$ burns at least $\chi_{\mathcal{C}}(\alpha)$ units of welfare.

Proof of Lemma A.1. Fix $\alpha \geq \alpha_{\text {ind }}$. We start by showing that (26) has a unique non-negative solution if one exists. Lemma B.4 shows that $g$ is increasing and hence invertible on the interval $\left[\alpha_{\text {ind }}, 1\right]$. Let $g^{-1}$ denote the inverse on $\left[\alpha_{\text {ind }}, 1\right]$ and define the function $\hat{\alpha}(x):=g^{-1}(x+g(\alpha))$ for $x \geq 0$. It is easy to check that $\hat{\alpha}(x) \geq \alpha$ and $g_{\alpha}^{-1}(x)=\frac{1}{\gamma}(z(\hat{\alpha}(x)-z(\alpha))$ for any $x \geq 0$. It follows from the formula for the inverse of the derivative that

$$
\hat{\alpha}^{\prime}(x)=\frac{1}{g^{\prime}\left(g^{-1}(x+g(\alpha))\right)}=\frac{1}{g^{\prime}\left(\hat{\alpha}_{x}(\alpha)\right)}>0,
$$

where we have used that $\hat{\alpha}(x) \geq \alpha \geq \alpha_{\text {ind }}$. Since $\hat{\alpha}(x) \geq \alpha_{\text {ind }} \geq \frac{1}{\mathrm{e}}$ and $z$ is increasing on $\left[\frac{1}{\mathrm{e}}, 1\right]$, it follows form the chain rule that $\left(g_{\alpha}^{-1}\right)^{\prime}(x)=\frac{1}{\gamma} z^{\prime}(\hat{\alpha}(x)) \hat{\alpha}^{\prime}(x)>0$. Since $g_{\alpha}^{-1}$ is strictly increasing, the right-hand side of (26) is strictly decreasing in $\chi$. Thus, if the right-hand side is lower than the left-hand side for $\chi=0$, there exists no non-negative solution. If the right-hand side is greater than or equal to the left-hand side for $\chi=0$, there exists precisely one non-negative solution. The fact that $\chi_{\mathcal{C}}(\alpha)$ is a lower bound for welfare burning in an accepting equilibrium $(b, s)$ with contributing banks $\mathcal{C}$ and $\alpha=\bar{\alpha}(b, s, 1)$ now follows from Lemma C.3.

Lemmas 3.8 and 3.9 together imply that any bail-in satisfying Conditions (i)-(iv) in Defini- 
tion 3.2 , if accepted, induces welfare losses

$$
W_{\mathcal{C}}(\alpha):=W_{P}-g\left(\alpha_{P}\right)+g(\alpha)-\lambda \sum_{i \in \mathcal{C}} \eta^{i}(\alpha, 0)+\chi_{\mathcal{C}}(\alpha)
$$

The following lemma establishes that this is a lower bound for welfare losses that can be attained by a bail-in with contributing banks $\mathcal{C}$ that induces asset price $\alpha$.

Lemma C.3. Let $(b, s)$ be a complete feasible bail-in proposal with accepting equilibrium a. Let $\mathcal{C}:=\left\{i \mid b^{i} 1_{\left\{a^{i}=1\right\}}>0\right\}$ and denote $\alpha=\bar{\alpha}(b, s, a)$ for the sake of brevity. Then $W_{\lambda}(b, s, a) \geq W_{\mathcal{C}}(\alpha)$ and the inequality binds if and only if $(b, s) \in \Xi(\mathcal{C}, \alpha)$.

Proof. Let $(b, s)$ be a complete feasible bail-in proposal with accepting equilibrium $a$. By Lemma C.1, we may assume without loss of generality that $b^{i} s^{i}=0$ and $a^{i}=1$ for every bank $i$. It follows in the same way as in the proof of Lemma 3.9 that $b^{i} \leq \eta^{i}(\alpha, e)$ for each $i \in \mathcal{C}$ and $s^{i} \geq s^{i}(\alpha, e)$ for each $i \notin \mathcal{C}$. Therefore, Conditions 1 and 2 of Definition 3.2 are satisfied.

Define the bail-in proposal $(\tilde{b}, \tilde{s})$ by setting $\tilde{b}^{i}=\max \left(b^{i}, \eta^{i}(\alpha, 0)\right)$ for each bank $i \in \mathcal{C}$ and $\tilde{s}^{i}=\min \left(s^{i}, s_{0}^{i}\right)$ for each bank $i \notin \mathcal{C}$. It follows as in the proof of Lemma 3.9 that $\alpha(\tilde{b}, \tilde{s}, 1)=\alpha$ and

$$
W_{\lambda}(b, s, 1)=W_{\lambda}(\tilde{b}, \tilde{s}, 1)+\chi=W_{P}-g\left(\alpha_{P}\right)+g(\alpha)-\lambda \sum_{i \in \mathcal{C}} \eta^{i}(\alpha, 0)+\chi,
$$

where we denote $\chi=\lambda \sum_{i=1}^{n}\left(s^{i}-s_{0}^{i}\right)^{+}+\lambda \sum_{i \in \mathcal{C}}\left(\eta^{i}(\alpha, 0)-b^{i}\right)^{+}$, and we have used Lemma 3.9 in the second equation. To show the first statement, it thus remains to show that $\chi \geq \chi_{\mathcal{C}}(\alpha)$.

For any bank $i \in \mathcal{C}$, let $a_{-i}$ denote the response vector where every bank but bank $i$ accepts the proposal. Set $\alpha_{-i}=\bar{\alpha}\left(b, s, a_{-i}\right)$. Welfare losses in this response are equal to

$$
\begin{aligned}
W_{\lambda}\left(b, s, a_{-i}\right) & =W_{\lambda}(b, s, 1)-g(\alpha)+g\left(\alpha_{-i}\right)+\lambda \min \left(b^{i}, b_{0}^{i}\right) \\
& =W_{P}-g\left(\alpha_{P}\right)-g(\alpha)+g\left(\alpha_{-i}\right)-h_{\mathcal{C} \backslash\{i\}}(\alpha)+\chi .
\end{aligned}
$$

Solving for $\chi$ and using Condition 1 of Lemma 3.5, we obtain

$$
\chi \geq W_{N}-W_{P}+g\left(\alpha_{P}\right)+g(\alpha)-g\left(\alpha_{-i}\right)+h_{\mathcal{C} \backslash\{i\}}(\alpha) .
$$

It follows from t35 that $\alpha \ell^{i}=\left(b^{i}-b_{0}^{i}\right)^{+}$, and hence

$$
-\frac{\alpha_{-i} \ln \left(\alpha_{-i}\right)}{\gamma}=-\frac{\alpha \ln (\alpha)}{\gamma}-\left(b^{i}-b_{0}^{i}\right)^{+} \geq-\frac{\alpha \ln (\alpha)}{\gamma}-\bar{b}^{i} .
$$

Multiplying (38) by $-\gamma$ and applying $g \circ z^{-1}$, we obtain $g\left(\alpha_{-i}\right)-g(\alpha) \leq g_{\alpha}\left(\bar{b}^{i}\right)$. In conjunction with (37), this yields

$$
\chi \geq W_{N}-W_{P}+g\left(\alpha_{P}\right)+h_{\mathcal{C} \backslash\{i\}}(\alpha)-g_{\alpha}\left(\bar{b}^{i}\right) .
$$


Next, solving (37) for $g\left(\alpha_{-i}\right)-g(\alpha)$ and applying $g_{\alpha}^{-1}$, we obtain

$$
\left(b^{i}-b_{0}^{i}\right)^{+}=\frac{\alpha_{-i} \ln \left(\alpha_{-i}\right)-\alpha \ln (\alpha)}{\gamma} \geq g_{\alpha}^{-1}\left(W_{N}-W_{P}+g\left(\alpha_{P}\right)+h_{\mathcal{C} \backslash\{i\}}(\alpha)-\chi\right) .
$$

Summing over all $i \in \mathcal{C}$ yields

$$
\begin{aligned}
-\frac{\alpha \ln (\alpha)}{\gamma} & =\sum_{i=1}^{n}\left(\left(s_{0}^{i}-s^{i}\right)^{+}+\left(b^{i}-b_{0}^{i}\right)^{+}\right) \geq \sum_{i \in \mathcal{C}}\left(b^{i}-b_{0}^{i}\right)^{+} \\
& \geq \sum_{i \in \mathcal{C}} g_{\alpha}^{-1}\left(W_{N}-W_{P}+g\left(\alpha_{P}\right)+h_{\mathcal{C} \backslash\{i\}}(\alpha)-\chi\right) .
\end{aligned}
$$

Since $\chi_{\mathcal{C}}(\alpha)$ is the smallest value $\chi^{\prime} \geq 0$ that satisfies (39) for all $i \in \mathcal{C}$ and (40), it follows that $\chi_{\mathcal{C}}(\alpha) \leq \chi$. This concludes the proof that $W_{\lambda}(b, s, a) \geq W_{\mathcal{C}}(\alpha)$.

Note that this lower bound holds with equality if and only if $\chi=\chi_{\mathcal{C}}(\alpha)$, i.e., Condition (iv) in Definition 3.2 is satisfied. It remains to show that $(b, s)$ satisfies the other four conditions. We have already argued that Conditions (i) and (ii) are satisfied. Equation (35) implies that

$$
\ell^{i}(b, s, a)=\frac{1}{\alpha}\left(\left(s_{0}^{i}-s^{i}\right)^{+}+\left(b^{i}-b_{0}^{i}\right)^{+}\right) .
$$

It follows from (1) and 41) that Condition (iii) is satisfied. Finally, Condition 1 of Lemma 3.5 for $i \in \mathcal{C}$ implies that Condition (v) holds. This concludes the proof.

Lemma C.3 shows that welfare losses $W_{\mathcal{C}}(\alpha)$ are attained only by bail-ins in $\Xi(\mathcal{C}, \alpha)$. The following lemma shows that the converse is true as well if $\alpha \geq \frac{1}{\mathrm{e}}$.

Lemma C.4. For any $\mathcal{C}$ and any $\alpha \geq \frac{1}{\mathrm{e}}$, any $(b, s) \in \Xi(\mathcal{C}, \alpha)$ is a complete feasible bail-in proposal with $W_{\lambda}(b, s, 1)=W_{\mathcal{C}}(\alpha)$ that admits response vector $1=(1, \ldots, 1)$ in equilibrium. Moreover, if $W_{\mathcal{C}}(\alpha)<W_{N}$, then $1=(1, \ldots, 1)$ is an accepting equilibrium.

Proof. Fix $(b, s) \in \Xi(\mathcal{C}, \alpha)$. It follows along the same lines as in the proof of Lemma G.1 that $(b, s)$ is a complete feasible bail-in with $\bar{\alpha}(b, s, 1)=\alpha$. Condition (i) in Definition 3.2 implies that Condition 2 in Lemma 3.5 is satisfied for every bank $i \in \mathcal{C}$ in the response vector 1 . It follows from Lemma 3.8 and Condition (iv) Definition 3.2 that $W_{\lambda}(b, s, 1)=W_{\mathcal{C}}(\alpha)$. Condition (v) in Definition 3.2 thus implies that Condition 1 of Lemma 3.5 is satisfied for every bank $i \in \mathcal{C}$. Therefore, an application of Lemma 3.5 shows that 1 is an equilibrium response. It is immediate that 1 is an accepting equilibrium if $W_{\mathcal{C}}(\alpha)<W_{N}$.

Lemma C.5 shows that the equilibrium bail-in contributors are the banks with the largest exposure to contagion effects.

Lemma C.5. For any bail-in proposal $(b, s)$, let $\ell(b, s)$ denote the induced vector of liquidations when every bank accepts the proposal. For any vector $\ell$, let $\mathcal{C}(\ell)$ be defined as in Theorem 3.10. 
Suppose that there exists $\mathcal{C}$ such that in any subgame Pareto-efficient equilibrium, a bail-in from $\Xi(\mathcal{C}, \alpha)$ is implemented. Then $\mathcal{C}=\mathcal{C}(\ell(b, s))$ for any $(b, s) \in \Xi(\mathcal{C}, \alpha)$.

Proof. Let $\mathcal{C}$ be such that in any subgame Pareto-efficient equilibrium, a bail-in from $\Xi(\mathcal{C}, \alpha)$ is implemented. Fix $(b, s) \in \Xi(\mathcal{C}, \alpha)$ and abbreviate $\ell=\ell(b, s)$. Suppose towards a contradiction that there exists a pair of banks $\left(i_{0}, j_{0}\right) \in \mathcal{C}^{c} \times \mathcal{C}$ such that $\eta^{i_{0}}(\alpha, \ell)>\eta^{j_{0}}(\alpha, \ell)$. Let $\tilde{\mathcal{C}}:=\mathcal{C} \backslash\left\{j_{0}\right\} \cup\left\{i_{0}\right\}$ and define a bail-in $(\tilde{b}, \tilde{s})$ by setting $\tilde{b}^{i_{0}}=\eta^{i_{0}}(\alpha, \ell), \tilde{s}^{i_{0}}=0, \tilde{b}^{j_{0}}=0, \tilde{s}^{j_{0}}=0$, as well as $\tilde{b}^{i}=b^{i}$ and $\tilde{s}^{i}=s^{i}$ for any other bank $i \notin\left\{j_{0}\right\} \cup\left\{i_{0}\right\}$.

We will first show that $(\tilde{b}, \tilde{s})$ is a complete, feasible bail-in proposal. By definition of $\ell$, the shortfall of any bank $i$ in the bail-in $(b, s)$ is $\alpha \ell^{i}$. Let $\tilde{\sigma}^{i}:=\left(L^{i}+w^{i}+\tilde{b}^{i}-c^{i}-\tilde{s}^{i}-(\pi L)^{i}\right)^{+}$ denote the shortfall of bank $i$ in bail-in $(\tilde{b}, \tilde{s})$. Since $\tilde{b}^{i}=b^{i}$ and $\tilde{s}^{i}=s^{i}$ for every $i \notin\left\{i_{0}, j_{0}\right\}$, it follows that $\tilde{\sigma}^{i}=\alpha \ell^{i}$ for every such bank $i$. Since $j_{0}$ makes a positive net contribution $b^{j_{0}}-s^{j_{0}}$ to the bail-in $(b, s)$ by Condition 1 of Lemma 3.5, it follows that $\tilde{\sigma}^{j_{0}} \leq\left(L^{j_{0}}+w^{j_{0}}+b^{j_{0}}-c^{j_{0}}-\right.$ $\left.s^{j_{0}}-(\pi L)^{j_{0}}\right)^{+}=\alpha \ell^{j_{0}}$ with strict inequality if $\ell^{j_{0}}>0$. The definition of $\eta(\alpha, \ell)$ in 16$)$ implies that $\tilde{b}^{i_{0}} \leq\left(c^{i_{0}}+\alpha \ell^{i_{0}}+(\pi L)^{i_{0}}-w^{i_{0}}-L^{i_{0}}\right)^{+}$, and hence $\tilde{\sigma}^{i_{0}} \leq \alpha \ell^{i_{0}}$ also for bank $i_{0}$. We conclude that $\tilde{\sigma}^{i} \leq \alpha \ell^{i} \leq e^{i}$ for every bank $i$, hence $(\tilde{b}, \tilde{s})$ is a complete feasible bail-in proposal.

Since the total shortfall is smaller in $(\tilde{b}, \tilde{s})$ than in $(b, s)$, it follows that $\tilde{\alpha}:=\alpha(\tilde{b}, \tilde{s}, 1) \geq \alpha$. Observe further that $\eta^{i_{0}}(\alpha, \ell)>\eta^{j_{0}}(\alpha, \ell) \geq b^{j_{0}}>0$ implies $s^{i_{0}}(\alpha, \ell)=0$ and hence $s^{i_{0}}=0=\tilde{s}^{j_{0}}$. It also implies that $\tilde{b}^{i_{0}}>b^{j_{0}}$, which yields

$$
W_{\lambda}(\tilde{b}, \tilde{s}, 1)=W_{\lambda}(b, s, 1)-(\alpha-\tilde{\alpha}) \sum_{i=1}^{n} e^{i}-\lambda\left(\tilde{b}^{i_{0}}-b^{j_{0}}\right)<W_{\lambda}(b, s, 1) .
$$

Because 1 is an accepting equilibrium for $(b, s)$, this shows that $W_{\lambda}(\tilde{b}, \tilde{s}, 1)<W_{N}$.

For any $i \in \tilde{\mathcal{C}}$, let $a_{-i}$ denote the response vector by the banks where every bank but $i$ accepts the proposal. Observe that $W_{\lambda}\left(\tilde{b}, \tilde{s}, a_{-i_{0}}\right)=W_{\lambda}\left(b, s, a_{-j_{0}}\right) \geq W_{N}$ by Condition 1 of Lemma 3.5 . For any $i \in \tilde{\mathcal{C}} \backslash\left\{i_{0}\right\}$, let $\tilde{\alpha}_{-i}=\bar{\alpha}\left(\tilde{b}, \tilde{s}, a_{-i}\right)$ and $\alpha_{-i}=\bar{\alpha}\left(b, s, a_{-i}\right)$ and observe that

$$
-\frac{\tilde{\alpha}_{-i} \ln \left(\tilde{\alpha}_{-i}\right)}{\gamma}+\frac{\alpha_{-i} \ln \left(\alpha_{-i}\right)}{\gamma}=\sum_{j \neq i} \tilde{\sigma}^{j}-\sum_{j \neq i} \alpha \ell^{j}=\tilde{\sigma}^{i_{0}}+\tilde{\sigma}^{j_{0}}-\alpha\left(\ell^{i_{0}}+\ell^{j_{0}}\right) \leq 0 .
$$

Equation (42) implies that $\tilde{\alpha}_{-i} \geq \alpha_{-i}$. Moreover, since the difference in shortfall is the same as the difference of shortfalls between $(\tilde{b}, \tilde{s}, 1)$ and $(b, s, 1)$, it follows from concavity of $x \mapsto-x \ln (x)$ that $\tilde{\alpha}_{-i}-\alpha_{-i} \leq \tilde{\alpha}-\alpha$. This implies that

$$
W_{\lambda}\left(b, s, a_{-i}\right)-W_{\lambda}\left(\tilde{b}, \tilde{s}, a_{-i}\right) \leq W_{\lambda}(b, s, 1)-W_{\lambda}(\tilde{b}, \tilde{s}, 1)
$$

Define now a vector of subsidies $\hat{s} \geq \tilde{s}$ that burns additional welfare precisely equal to

$$
\chi:=\max _{i \in \mathcal{C}}\left(W_{\lambda}\left(b, s, a_{-i}\right)-W_{\lambda}\left(\tilde{b}, \tilde{s}, a_{-i}\right)\right) .
$$

This can be attained either by awarding additional subsidies $\chi / \lambda$ to a bank $i \notin \mathcal{C}$ with $\ell^{i}=0$ or 
by awarding additional subsidies $\frac{1}{\lambda}\left(\chi+\left(z^{-1}\left(\ln (\tilde{\alpha}) \tilde{\alpha}+\alpha \gamma \ell^{i}\right)-\tilde{\alpha}\right) \sum_{i=1}^{n} e^{i}\right)$ to a bank $i \in \mathcal{C}$. By Condition 1 of Lemma 3.5 for bail-in $(b, s)$, we have $W_{\lambda}\left(\tilde{b}, \hat{s}, a_{-i}\right) \geq W_{\lambda}\left(b, s, a_{-i}\right) \geq W_{N}$, showing that Condition 1 of Lemma 3.5 is also satisfied in $(\tilde{b}, \hat{s})$. Since $\alpha(\tilde{b}, \hat{s}, 1) \geq \tilde{\alpha} \geq \alpha$, it follows that also Condition 2 of Lemma 3.5 is satisfied in $(\tilde{b}, \hat{s})$ for every bank $i$. Finally, it follows from (43) that

$$
W_{\lambda}(\tilde{b}, \hat{s}, 1)=W_{\lambda}(\tilde{b}, \tilde{s}, 1)+\chi \leq W_{\lambda}(b, s, 1)<W_{N} .
$$

This shows that $a=(1, \ldots, 1)$ is an accepting equilibrium for $(\tilde{b}, \hat{s})$. Since no subset of accepting banks is an accepting equilibrium by Condition 2 of Lemma 3.5, it is the unique accepting equilibrium and hence also the unique subgame Pareto efficient continuation equilibrium by Lemma 3.7. Since $(b, s)$ is a subgame Pareto efficient equilibrium outcome, the regulator has no profitable deviations from $(\tilde{b}, \hat{s})$. This shows that $(\tilde{b}, \hat{s})$ is a subgame Pareto-efficient equilibrium outcome as well, contradicting the fact that contributions have to come from $\mathcal{C}$. We conclude that $C=\mathcal{C}(\ell)$.

Finally, we need one last technical result before we are able to prove Theorem 3.10 .

Lemma C.6. For any set of banks $\mathcal{C}$, the function $\chi_{\mathcal{C}}$ defined in Lemma $A .1$ is continuous on the interval $\left[\frac{1}{\mathrm{e}}, 1\right]$. Hence, $W_{\mathcal{C}}$ is continuous as well.

Proof. Fix an arbitrary set of banks $\mathcal{C}$. Since $g, \eta(\cdot, \ell)$, and $g_{\alpha}(x)$ are continuous in $\alpha$, it follows straight from the definition of $\chi_{\mathcal{C}}^{i}$ in Lemma A.1 that $\chi_{\mathcal{C}}^{i}$ is continuous in $\alpha$ for each $i$. For continuity of $\hat{\chi}_{\mathcal{C}}(\alpha)$, let $f_{\mathcal{C}}(\alpha, \chi)$ denote the function on the right-hand side of 26). As we have shown in the proof of Lemma A.1, $f_{\mathcal{C}}(\alpha, \chi)$ is strictly decreasing in $\chi$. By definition, $\hat{\chi}_{\mathcal{C}}(\alpha)$ is the smallest value $\chi \geq 0$ such that $f_{\mathcal{C}}(\alpha, \chi) \leq-z(\alpha) / \gamma$. On the set $O:=\left\{\alpha \in\left[\frac{1}{\mathrm{e}}, 1\right] \mid f_{\mathcal{C}}(\alpha, 0) \leq-z(\alpha) / \gamma\right\}$, it follows that $\hat{\chi}_{\mathcal{C}}(\alpha)=0$. On the complement of $O$, we have $f_{\mathcal{C}}\left(\alpha, \hat{\chi}_{\mathcal{C}}(\alpha)\right)=-z(\alpha) / \gamma$ by continuity of $f_{\mathcal{C}}(\alpha, \chi)$ in $\chi$. For any $\alpha \in O^{c}$, let $f_{\mathcal{C}}^{-1}\left(-\frac{z(\alpha)}{\gamma}\right)$ denote the pre-image of $-\frac{z(\alpha)}{\gamma}$ under $f_{\mathcal{C}}(\alpha, \cdot)$. Since $\hat{\chi}_{\mathcal{C}}(\alpha)$ is the unique solution to (26), the pre-image is unique, meaning that $f_{\mathcal{C}}(\alpha, \cdot)$ is invertible. It follows that $\hat{\chi}_{\mathcal{C}}(\alpha)=f_{\mathcal{C}}^{-1}\left(-\frac{z(\alpha)}{\gamma}\right)$ is continuous on $O^{c}$ being the composition of continuous functions. Continuity of $f_{\mathcal{C}}$ and $z$ implies that $\lim \hat{\chi}_{\mathcal{C}}(\alpha)=0$ as $\alpha \in O^{c}$ approaches the border of $O$ and hence $\hat{\chi}_{\mathcal{C}}(\alpha)$ is continuous everywhere. Finally, $\chi_{\mathcal{C}}$ is continuous being the maximum of continuous functions. Continuity of $W_{\mathcal{C}}$ now follows from the continuity of $g$ and $\eta^{i}(\cdot, 0)$ for any $i \in \mathcal{C}$.

Proof of Theorem 3.10. If the threat fails to be credible, then it follows from Proposition 3.3 that the regulator will implement an optimal public bailout as given in Lemma 5.1, either by proposing it in the first stage or by choosing it in the third stage after an arbitrary proposal in the first stage.

Suppose, therefore, that the threat is credible. Since $W_{\mathcal{C}}$ is continuous by Lemma C.6. $W_{\mathcal{C}}$ attains a minimum on the interval $\left[\frac{1}{\mathrm{e}}, 1\right]$. Let $A(\mathcal{C})$ denote the set of prices $\alpha$ that minimize $W_{\mathcal{C}}(\alpha)$. Let $\mathcal{C}_{*}$ denote the set of banks $\mathcal{C}$ that $\operatorname{minimizes} \min _{\alpha} W_{\mathcal{C}}(\alpha)$. For a generic choice of model parameters, the functions $\left(W_{\mathcal{C}}\right)_{\mathcal{C} \subseteq\{1, \ldots, N\}}$ are all distinct, and hence $\mathcal{C}_{*}$ is generically unique.

Let $\alpha_{*}:=\max \left\{\alpha \in A\left(\mathcal{C}_{*}\right)\right\}$. By Lemma C.4, any bail-in proposal $(b, s) \in \Xi\left(\mathcal{C}_{*}, \alpha\right)$ for any $\alpha \in A\left(\mathcal{C}_{*}\right)$ admits equilibrium response $1=(1, \ldots, 1)$, and it attains welfare losses $W_{\mathcal{C}_{*}}\left(\alpha_{*}\right)$. It follows as a consequence of Lemma G.5 in the online appendix that $W_{\mathcal{C}_{*}}\left(\alpha_{*}\right)<W_{N}$, and hence the 
response vector of unanimous acceptance is an accepting equilibrium for any $(b, s) \in \Xi\left(\mathcal{C}_{*}, \alpha\right)$ and any $\alpha \in A\left(\mathcal{C}_{*}\right)$ by Lemma C.4. By Condition 1 in Lemma 3.5, it is the unique accepting equilibrium. Since rejecting equilibria are subgame Pareto dominated by Lemma 3.7, the regulator is aware that any bail-in $(b, s) \in \Xi\left(\mathcal{C}_{*}, \alpha\right)$, for $\alpha \in A\left(\mathcal{C}_{*}\right)$, which he proposes in the first stage will be accepted in

equilibrium. Because no incentive-compatible bail-in can attain lower welfare losses by Lemmas C.2 and C.3, the regulator's only rational choice in stage 1 is to propose such a bail-in. Subgame Pareto efficiency then imposes that the regulator proposes a bail-in from $\Xi\left(\mathcal{C}_{*}, \alpha_{*}\right)$. Finally, Lemma C.5 shows that $\mathcal{C}_{*}=\mathcal{C}(\ell(b, s))$ for any $(b, s) \in \Xi\left(\mathcal{C}_{*}, \alpha_{*}\right)$ if $\mathcal{C}_{*}$ is unique.

\section{Data Calibration Procedure}

A total of 48 banks participated in the stress test of the EBA. Values of various banks' balance sheet quantities are reported as of end 2017. For each bank, the EBA reports the exposures to other institutions, computed using an internal rating based (IRB) criterion as of end 2017. We take those exposures as a measure of the bank's total claims $A$ on all other banks in the network. Some of the smallest banks reported zero interbanking claims. We omit those from the analysis, leaving us with a total of 36 banks. To estimate interbank liabilities, we first compute the banks' total liabilities as the difference between their total asset holdings and their equity value $E$, both of which are reported in the EBA data. We then assume that each bank has the same ratio of interbank to total liabilities. We compute this ratio by imposing that the sum of interbank liabilities across all banks equals the sum of interbank claims. Since most quantities in our model depend on $c$ and $w$ only through their difference $c-w$, we set $c=E+\max (E+L-A, 0)$ and $w=E+\max (A-E-L, 0)$ so that the equity value of each bank corresponds to that implied from the EBA data, and we can apply a shock of size $c^{i} \geq E^{i}$ to any bank to induce a fundamental default. All raw data and estimated model quantities are summarized in Table 1. We set $\lambda=3$ and the recovery rate $\beta$ to $60 \%$. This value is in line with empirical estimates reported by Moody's; see Footnote 13 .

Finally, we discuss how we construct the sparse network $\pi_{s}$. We use an iterated greedy algorithm. In each step of the algorithm, we construct a ring network $j_{1} \rightarrow j_{2} \rightarrow \cdots \rightarrow j_{n} \rightarrow j_{1}$ as follows: Given $j_{1} \rightarrow \cdots \rightarrow j_{k}$, the creditor of $j_{k}$ is selected to be the bank with the smallest interbank claims among all banks in $\left\{j_{k+1}, \ldots, j_{n}\right\}$ whose interbank claims exceed $j_{k}$ 's interbank liabilities. If such a bank exists, all of $j_{k}$ 's liabilities are assigned to that bank. If there exists no such bank, $j_{k+1}$ is the bank with the largest interbank claims and we assign $\sum_{i} L^{j_{k+1} i}$ of $j_{k}$ 's liabilities to bank $j_{k+1}$, leaving the remainder of $j_{k+1}$ 's interbank liabilities to be assigned in the next iteration. Each ring starts with the bank whose unassigned interbank liabilities are the largest. This method is greedy because, for each considered bank, it assigns all its interbank liabilities to a single bank, if possible. An iterated construction using rings guarantees that the resulting network consists of only one connected component. 


\begin{tabular}{|c|c|c|c|c|c|c|}
\hline Bank Name & Total Claims & Equity & Interbank Claims $A^{i}$ & Interbank Liabilities $L^{i}$ & Senior Liabilities $w^{i}$ & Cash holdings $c^{i}$ \\
\hline Erste Group Bank AG & 147,449 & 15,368 & 10,756 & 12,394 & 15,368 & 32,374 \\
\hline Raiffeisen Bank International AG & 61,499 & 9,839 & 5,416 & 4,846 & 9,839 & 19,108 \\
\hline Belfius Banque SA & 127,440 & 8,141 & 35,597 & 11,192 & 24,404 & 8,141 \\
\hline KBC Group NV & 226,455 & 16,552 & 17,128 & 19,692 & 16,552 & 35,668 \\
\hline Danske Bank & 273,199 & 20,302 & 14,926 & 23,725 & 20,302 & 49,403 \\
\hline OP Financial Group & 91,467 & 9,973 & 7,299 & 7,645 & 9,973 & 20,292 \\
\hline BNP Paribas & $1,012,707$ & 84,417 & 63,604 & 87,088 & 84,417 & 192,318 \\
\hline Group Credit Mutuel & 430,308 & 45,578 & 44,606 & 36,093 & 45,578 & 82,643 \\
\hline Groupe Credit Agricole & $1,047,925$ & 84,292 & 97,114 & 90,404 & 84,292 & 161,874 \\
\hline Societe Generale S.A. & 642,940 & 49,514 & 53,400 & 55,672 & 49,514 & 101,300 \\
\hline Bayerische Landesbank & 193,192 & 9,393 & 22,731 & 17,243 & 9,393 & 13,298 \\
\hline Commerzbank AG & 314,214 & 25,985 & 42,564 & 27,040 & 25,985 & 36,446 \\
\hline Deutsche Bank AG & 758,140 & 57,631 & 58,015 & 65,719 & 57,631 & 122,966 \\
\hline DZ Bank AG & 202,301 & 19,923 & 35,800 & 17,110 & 19,923 & 21,156 \\
\hline Landsesbank Baden-Wurttemberg & 206,824 & 12,795 & 57,434 & 18,203 & 39,230 & 12,795 \\
\hline Landsesbank Hessen-Thuringen Girozentrale & 122,115 & 8,180 & 15,767 & 10,688 & 8,180 & 11,281 \\
\hline Allied Irish Banks Group plc & 48,157 & 11.028 & 10.064 & 3,484 & 11,028 & 15,475 \\
\hline Bank of Ireland Group plc & 68,264 & 7,617 & 4,537 & 5,689 & 7,617 & 16,386 \\
\hline Intesa Sanpolo S.p.A. & 309,144 & 43,466 & 36,125 & 24,924 & 43,466 & 75,731 \\
\hline UniCredit S.p.A. & 309,144 & 43,466 & 36,125 & 24,924 & 54,703 & 101,448 \\
\hline ABN AMRO Group N.V. & 367,487 & 19,618 & 14,942 & 32,635 & 19,618 & 56,929 \\
\hline Cooperatieve Rabobank U.A. & 547,353 & 37,204 & 14,461 & 47,860 & 37,204 & 107,807 \\
\hline ING Groep N.V. & 780,776 & 50,325 & 76,469 & 68,528 & 50,325 & 92,709 \\
\hline Banco Bilbao Vizcaya Argentaria S.A. & 276,960 & 46,980 & 75,226 & 21,575 & 53,650 & 46,980 \\
\hline Banco de Sabadell S.A. & 108,282 & 11,111 & 1,559 & 9,116 & 11,111 & 29,779 \\
\hline Banco Santander S.A. & 565,109 & 77,283 & 36,878 & 45,765 & 77,283 & 163,453 \\
\hline Nordea Bank - group & 437,347 & 28,008 & 40.127 & 38,402 & 28,008 & 54,291 \\
\hline Skandinaviska Enskilda Banken - group & 209,082 & 13,452 & 14,944 & 18,353 & 13,452 & 30,313 \\
\hline Svenska Handelsbanken - group & 253,639 & 12,954 & 7,339 & 22,580 & 12,954 & 41,149 \\
\hline Swedbank - group & 202,830 & 11,356 & 6,522 & 17,963 & 11,356 & 34,153 \\
\hline Barclays Plc & 562,002 & 60,765 & 49,797 & 47,024 & 60,765 & 118,757 \\
\hline HSBC Holdings Plc & $1,322,909$ & 125,976 & 117,004 & 112,291 & 125,976 & 247,239 \\
\hline Lloyds Banking Group Plc & 590,827 & 40,948 & 8,817 & 51,587 & 40,948 & 124,666 \\
\hline
\end{tabular}

Table 1: Results based on data from the 2018 EBA stress test. All numbers are reported in million dollars. 


\title{
Online Appendix to "Bail-ins and Bail-outs: Incentives, Connectivity, and Systemic Stability"
}

\author{
Benjamin Bernard, Agostino Capponi, and Joseph E. Stiglitz
}

This online appendix contains the characterization of the equilibrium asset price and an algorithm for computing the equilibrium bail-in of Theorem 3.10. It also contains proofs for the results in Sections 4 and 5, as well as some auxiliary results omitted from the main text.

\section{E Characterization of Equilibrium Asset Price}

This appendix provides more details on the results presented in Section 3 , characterizing the equilibrium asset price $\alpha_{*}$ in Theorem 3.10 as well as the equilibrium amount of welfare burnt. We also present an iterative algorithm to compute $\mathcal{C}_{*}$ and $\alpha_{*}$.

As in the main body, we begin by considering bail-ins where each bank $i$ in some set $\mathcal{C}$ contributes $\eta^{i}(\alpha, \ell)$ and each bank $i \notin \mathcal{C}$ receives subsidies $s^{i}(\alpha, \ell)$ for a liquidation decision $\ell$ that induces asset price $\alpha$, where $\eta(\alpha, \ell)$ and $s(\alpha, \ell)$ are defined in (16) and (17), respectively. For ease of reference, the set of all such bail-ins is formalized in the following definition.

Definition E.1. Let $\Xi_{*}(\mathcal{C}, \alpha)$ denote the set of all bail-ins $(b, s)$ satisfying

(i) $\eta^{i}(\alpha, 0) \leq b^{i}-s^{i} \leq \eta^{i}(\alpha, e)$ for every $i \in \mathcal{C}$,

(ii) $b^{i}=0$ and $s^{i}(\alpha, e) \leq s^{i} \leq s^{i}(\alpha, 0)$ for every $i \notin \mathcal{C}$,

(iii) $\sum_{i=1}^{n}\left(s^{i}(\alpha, 0)-s^{i}\right)+\sum_{i \in \mathcal{C}}\left(b^{i}-\eta^{i}(\alpha, 0)\right)=-\frac{\alpha \ln (\alpha)}{\gamma}$.

As stated by Lemma 3.9 , welfare losses are minimized by bail-ins in $\Xi_{*}(\mathcal{C}, \alpha)$ among individually incentive-compatible bail-ins with contributing banks in $\mathcal{C}$. Since $\min \left(\eta(\alpha, \ell), b_{0}\right)=\eta(\alpha, 0)$ for any $\ell$, Lemma 3.8 shows that the welfare impact of the banks' contributions is equal to

$$
h_{\mathcal{C}}(\alpha):=\lambda \sum_{i \in \mathcal{C}} \eta^{i}(\alpha, 0)-g(\alpha)
$$

The welfare impact of contributions by banks in $\mathcal{C}$ depends on the liquidation decision only through the induced asset price. Thus, for a fixed set $\mathcal{C}$, the regulator performs a one-dimensional optimization over the induced asset price. The following lemma establishes that $g$ is convex and $h_{\mathcal{C}}(\alpha)$ is concave for any set $\mathcal{C}$, making this optimization simple.

Lemma E.1. For each set of banks $\mathcal{C}$, the function $h_{\mathcal{C}}$ defined in (44) is strictly concave with maximizer $\hat{\alpha}_{\mathcal{C}} \geq \alpha_{\text {ind }}$. Moreover, if $\mathcal{C} \subseteq \mathcal{C}^{\prime}$, then $\hat{\alpha}_{\mathcal{C}} \leq \hat{\alpha}_{\mathcal{C}^{\prime}}$. 
For a set of contributing banks $\mathcal{C}$, the price $\hat{\alpha}_{\mathcal{C}}$ is the break-even point where the regulator's marginal benefit from asset liquidation equals the marginal benefit from taxpayer contributions. The last statement of Lemma B.4 shows that the regulator's indifference price increases when he includes more banks into the bail-in consortium. Since $\hat{\alpha}_{\emptyset}=\alpha_{\text {ind }}$ by (44), this shows that the regulator's indifference price is higher in any bail-in than in a public bailout.

The regulator would like to induce asset price $\hat{\alpha}_{\mathcal{C}}$ if it is possible to do so in equilibrium. It may not be possible if banks do not liquidate a sufficient quantity of assets to drive the asset price down to $\hat{\alpha}_{\mathcal{C}}$ or if the no-free-riding constraint fails to hold for the resulting bail-in. The following lemma analyzes the maximum liquidation constraint, characterizing the lowest asset price $\alpha_{\mathcal{C}}$ that can be attained by any bail-in with contributing banks $\mathcal{C}$.

Lemma E.2. For any set of banks $\mathcal{C}$, the equation

$$
\alpha=\exp \left(-\gamma \sum_{i=1}^{n} \frac{1}{\alpha}\left(L^{i}+w^{i}+\eta^{i}(\alpha, e) 1_{\{i \in \mathcal{C}\}}-c^{i}-s^{i}(\alpha, e)-(\pi L)^{i}\right)^{+}\right)
$$

admits at least one fixed point. Let $\alpha_{\mathcal{C}}$ denote the largest fixed point of 45 . Then $\alpha(b, s, 1) \geq \alpha_{\mathcal{C}}$ for any complete, feasible, and individually incentive-compatible bail-in with contributing banks in $\mathcal{C}$. Moreover, $\alpha_{\mathcal{C}} \geq \frac{1}{\mathrm{e}}$ and $\alpha_{\mathcal{C}}$ can be attained in any bail-in from the set $\Xi_{*}\left(\mathcal{C}, \alpha_{\mathcal{C}}\right)$.

Together, Lemmas E.1 and E.2 show that in the absence of any free-riding, the regulator would optimally attain asset price $\alpha_{\mathcal{C}}^{*}:=\max \left(\min \left(\hat{\alpha}_{\mathcal{C}}, 1\right), \alpha_{\mathcal{C}}\right)$ for some set of contributing banks $\mathcal{C}$. The characterization in the following Theorem takes into account the free-riding constraints.

Theorem E.3. For any integer $k$ let $\mathcal{C}_{k}$ denote the set $\mathcal{C}$ of size $k$ that maximizes $h_{\mathcal{C}}\left(\alpha_{\mathcal{C}}^{*}\right)$. Set $W_{k}:=W_{P}-g\left(\alpha_{P}\right)-h_{\mathcal{C}_{k}}\left(\alpha_{\mathcal{C}_{k}}^{*}\right)$ and and $m:=\min \left(k \mid W_{k}<W_{N}\right)$. For any set of banks $\mathcal{C}$, let

$$
\alpha_{\mathcal{C}}^{\prime}:=\max \underset{\alpha \in\left[\alpha_{\mathcal{C}}, 1\right]}{\arg \min }\left(\hat{\chi}_{\mathcal{C}}(\alpha)-h_{\mathcal{C}}(\alpha)\right)
$$

denote $\tilde{\alpha}_{\mathcal{C}}^{i}:=\sup \left\{\alpha \mid \chi_{\mathcal{C}}^{i}(\alpha)>0\right\}$, and set $\tilde{\alpha}_{\mathcal{C}}:=\alpha_{\mathcal{C}}^{*} \vee \max _{i \in \mathcal{C}} \tilde{\alpha}_{\mathcal{C}}^{i}$. Define

$$
\alpha(\mathcal{C}):= \begin{cases}\alpha_{\mathcal{C}}^{*} & \text { if }|\mathcal{C}| \leq m \\ \tilde{\alpha}_{\mathcal{C}} & \text { if }|\mathcal{C}|>m \text { and } \hat{\chi}_{\mathcal{C}}\left(\tilde{\alpha}_{\mathcal{C}}\right)=0 \\ \alpha_{\mathcal{C}}^{\prime} & \text { otherwise }\end{cases}
$$

If $W_{N} \leq W_{P}$, then $\alpha_{*}=\alpha\left(\mathcal{C}_{*}\right)$ in any subgame Pareto efficient equilibrium. In particular, welfare burning is used only if $\left|\mathcal{C}_{*}\right|>m$ and $\hat{\chi}_{\mathcal{C}}\left(\tilde{\alpha}_{\mathcal{C}}\right)>0$.

For sufficiently small consortia of contributing banks, there is no incentive to free-ride and welfare is maximized at the unconstrained optimum $\alpha_{\mathcal{C}}^{*}$. For larger consortia, welfare is maximized at the asset price $\tilde{\alpha}_{\mathcal{C}}$ closest to the unconstrained optimum $\alpha_{\mathcal{C}}^{*}$, at which no welfare burning is necessary if the total liquidation implied by the no free-riding constraint is consistent with asset 
price $\tilde{\alpha}_{\mathcal{C}}$, that is, it does not depress the asset price below $\tilde{\alpha}_{\mathcal{C}} 1$ If the total liquidation implied by the no free-riding constraint is inconsistent with the asset price $\tilde{\alpha}_{\mathcal{C}}$, the regulator requests lower contributions from banks which induce asset price $\alpha_{\mathcal{C}}^{\prime}$ and require burning of $\hat{\chi}_{\mathcal{C}}(\alpha)$ units of welfare. At asset price $\alpha_{\mathcal{C}}^{\prime}$, the consistency requirement binds. This implies the following corollary.

Corollary E.4. Let $\mathcal{C}_{*}$ and $\alpha_{*}$ be as in Theorem E.3. Then $\chi_{\mathcal{C}_{*}}\left(\alpha_{*}\right)=\hat{\chi}_{\mathcal{C}}\left(\alpha_{*}\right)$.

For implementation purposes, one needs to worry that the welfare loss minimization problem yields a local minimum instead of the global minimum $\alpha_{\mathcal{C}}^{\prime}$. The following Lemma states relatively weak conditions on the parameters, under which the optimization is quasi-convex and the unique local minimum is the global minimum.

Lemma E.5. Let $m$ be defined as in Theorem E.3. Set $\alpha(i):=\alpha_{N}+\left(b_{0}-\pi\left(L-p_{N}\right)\right)^{i} / e^{i}$ for any bank $i$. For any $\mathcal{C}$, define $\mathcal{C}(\alpha):=\left\{i \in \mathcal{C} \mid \alpha(i) \leq \alpha, h_{\mathcal{C} \backslash\{i\}}(\alpha) \geq W_{P}-W_{N}+g\left(\alpha_{P}\right)+\hat{\chi}_{\mathcal{C}}(\alpha)\right\}$ and $W_{\mathcal{C}}(\alpha)=W_{P}-g\left(\alpha_{P}\right)-h_{\mathcal{C}}(\alpha)+\chi_{\mathcal{C}}(\alpha)$. Suppose that

$$
\left(\lambda-\frac{1}{\sqrt{2}}\right)\left(1+\frac{1}{\mathrm{e}}\right)<\gamma \sum_{i=1}^{n} e^{i}
$$

holds. If $\left|\mathcal{C}\left(\tilde{\alpha}_{\mathcal{C}}\right)\right|=1$, then $W_{\mathcal{C}}$ is quasi-convex and $\alpha_{\mathcal{C}}^{\prime} \in\left[\alpha_{\text {ind }}, \tilde{\alpha}_{\mathcal{C}}\right]$. If $\left|\mathcal{C}\left(\tilde{\alpha}_{\mathcal{C}}\right)\right| \geq 2$, then $W_{\mathcal{C}}$ is minimized at $\alpha=1$ and if $\left|\mathcal{C}\left(\tilde{\alpha}_{\mathcal{C}}\right)\right|=0$, then $W_{\mathcal{C}}$ is minimized at $\tilde{\alpha}_{\mathcal{C}}$. In particular, welfare burning is used only if $\left|\mathcal{C}\left(\tilde{\alpha}_{\mathcal{C}_{*}}\right)\right|=1$ or $\alpha_{*}=1$.

If the weight of taxpayer contributions in the regulator's objective function is not too high or if the price is sufficiently elastic, then welfare losses are minimized either at $\alpha=1$ or by the unique local minimum of a quasi-convex function 2 If the conditions of Lemma E.5 are satisfied, then the minimization of $W_{\mathcal{C}}$ for $\mathcal{C}$ such that $|\mathcal{C}|>m$ is simplified as follows. First, one computes $\tilde{\alpha}_{\mathcal{C}}$ and $\mathcal{C}\left(\tilde{\alpha}_{\mathcal{C}}\right)$. If $\mathcal{C}\left(\tilde{\alpha}_{\mathcal{C}}\right)$ is not a singleton, $W_{\mathcal{C}}$ is minimized analytically by Lemma E.5. Only if $\mathcal{C}\left(\tilde{\alpha}_{\mathcal{C}}\right)$ is a singleton, a numerical minimization needs to be performed. By quasi-convexity, the global minimum can be computed quickly through a binary search.

Theorem E.3 implies an algorithm that can be used to find the optimal set $\mathcal{C}_{*}$ of contributing banks. It suggests that the regulator performs an iteration over the size of the consortia of contributing banks, similarly as in Theorem 5.4. Different from the setting of Section 5 in the presence of price impact there is no global order according to which the regulator includes banks into the bail-in consortium. Rather, the set of most exposed banks changes as the regulator includes more banks into the consortium because the size of their contributions may impact their liquidation decisions, and thus affect the asset price. The set of liquidation decisions $\ell_{k}$, the asset price $\alpha_{k}$, and the set $\mathcal{C}_{k}$ of contributing banks for each $k$ is a fixed point of 45 such that $\ell_{k}$ induces asset price $\alpha_{k}$, contributions $\eta^{i}\left(\alpha_{k}, \ell_{k}\right)$ are maximized by banks in $\mathcal{C}_{k}$, and the contributions $\eta^{i}\left(\alpha_{k}, \ell_{k}\right)$

\footnotetext{
${ }^{1}$ At asset price $\alpha$, the no free-riding condition imposes that a contributing bank $i \in \mathcal{C}$ liquidates the smallest amount $x^{i} \geq 0$ for which $\lambda \eta^{i}(\alpha, 0)+g_{\alpha}\left(x^{i}\right) \geq W_{N}-W_{P}-g\left(\alpha_{P}\right)-h_{\mathcal{C}}(\alpha)$; see Lemma A.1 This liquidation amount is inconsistent with asset price $\alpha$ if and only if $\sum_{i \in \mathcal{C}} x^{i}>-\ln (\alpha) / \gamma$ because then, the required liquidation would depress the asset price below $\alpha$ by 11 .

${ }^{2}$ If $\left|\mathcal{C}\left(\tilde{\alpha}_{\mathcal{C}}\right)\right|=0$, then $W_{\mathcal{C}}$ locally coincides with the convex function $W_{P}-g\left(\alpha_{P}\right)-h_{\mathcal{C}}$, which is, in particular, quasi-convex.
} 
by banks in $\mathcal{C}_{k}$ at asset price $\alpha_{k}$ induce the liquidation vector $\ell_{k}$. Nevertheless, since $\eta^{i}(\alpha, \ell)$ is piecewise linear in $\alpha$, the set of banks most exposed to contagion does not change too frequently and one can use the set $\mathcal{C}_{k+1}^{\prime}$ of $k+1$ most-exposed banks using $\left(\alpha_{k}, \ell_{k}\right)$ as a starting point for constructing the set $\mathcal{C}_{k+1}$. If there exists $\ell^{\prime}$ with $\alpha\left(\ell^{\prime}\right)=\alpha\left(\mathcal{C}_{k+1}^{\prime}\right)$ such that $\mathcal{C}_{k+1}^{\prime}$ is the set of $k+1$ most exposed banks for $\left(\alpha\left(\ell^{\prime}\right), \ell^{\prime}\right)$, then $\mathcal{C}_{k+1}=\mathcal{C}_{k+1}^{\prime}$. Different from Theorem 5.4, the optimization does not stop after including $m+1$ banks: adding more banks into the consortium can increase welfare even after adding $m+1$ banks if the contributions by the banks require a smaller amount of asset liquidation. As a function of $k$, the asset price is decreasing until $m$ and increasing thereafter.

\section{F Existence, Monotonicity, and Differentiability of Asset Prices}

This section contains results related to the asset price, which are omitted from the main text. We show that $\alpha_{p}$, for a repayment vector $p$, is differentiable. We also show existence of the fixed point $\alpha_{\mathcal{C}}$ defined in Lemma E.2. Lastly, we provide an elementary construction of the asset price that allows us to conclude that $\alpha(b, s, 1) \geq \frac{1}{\mathrm{e}}$ for complete rescues $(b, s)$ in the proof of Lemma C.2.

Lemma F.1. Let $\alpha_{p}$ be defined as in Lemma 2.2. The asset price $\alpha_{p}$ is continuous in $L, \pi, c, w, e$, and $p$. Moreover, it is differentiable in these variables where $\mathcal{D}=\left\{i \mid L^{i}+w^{i}>c^{i}+\alpha_{p} e^{i}+(\pi p)^{i}\right\}$ and $\mathcal{I}=\left\{i \notin \mathcal{D} \mid L^{i}+w^{i}>c^{i}+(\pi p)^{i}\right\}$ are constant.

In the proof of Lemma F.1, we need the following auxiliary result, Lemma F.2, which is also invoked in the proof of Lemma C.2 in the main text.

Lemma F.2. Let $x, y \geq 0$ parametrize the function $f_{x, y}(\alpha)=\exp \left(-\frac{x}{\alpha}-y\right)$ and let $x_{0}=\exp (-y-1)$. The function $f_{x, y}$ has a fixed point on $(0,1]$ if and only if $x \leq x_{0}$. The fixed point is unique at $\alpha=\exp (-y)$ if $x=0$, it is unique at $\alpha=x_{0}$ if $x=x_{0}$, and otherwise there are two fixed points, one on $\left(0, x_{0}\right)$ and one on $\left(x_{0}, 1\right)$. The fixed point on $\left(0, x_{0}\right)$ is increasing in $x$, the fixed point on $\left(x_{0}, 1\right)$ is decreasing in $x$, and both are differentiable in $x$.

Proof. Fix $y \geq 0$. The statement is obvious if $x=0$. We thus restrict our attention to the case $x>0$. Then $f_{x, y}(1)<1$ and $\lim _{\alpha \rightarrow 0+} f_{x, y}(\alpha)=0$. Since $f_{x, y}$ is continuously differentiable on $(0,1]$ with $\lim _{\alpha \rightarrow 0+} f_{x, y}^{\prime}(\alpha)=0$, this implies that the graph of $f_{x, y}$ lies below the identity graph immediately to the right of 0 and immediately to the left of 1 . Observe next that

$$
f_{x, y}^{\prime \prime}(\alpha)=f_{x, y}^{\prime}(\alpha) \frac{x}{\alpha^{2}}-f_{x, y}(\alpha) \frac{2 x}{\alpha^{3}}=\frac{f_{x, y}(\alpha)}{\alpha^{4}}\left(x^{2}-2 x \alpha\right) .
$$

Therefore, $f_{x, y}$ is convex for sufficiently large $x$, which implies that the graph of $f_{x, y}$ is below the identity graph on the entire interval. In particular, $f_{x, y}$ has no fixed points for sufficiently large $x$.

Because $f_{x, y}$ is differentiable for any $x$ and the graph of $f_{x, y}$ changes continuously in $x$, the number of fixed points can change only at a point $x_{*}$ where the graph of $f_{x_{*}, y}$ and the identity graph touch, that is, where $f_{x_{*}, y}^{\prime}\left(\alpha_{0}\right)=1$ for a fixed point $\alpha_{0}$ of $f_{x_{*}, y}$. Moreover, for each such touching point $\alpha_{0}$, the number of fixed points can increase or decrease by at most 2 . 
Suppose that there exists $x_{*}$ with "touching point" $\alpha_{0}$. Then $1=f_{x_{*}, y}^{\prime}\left(\alpha_{0}\right)=f_{x_{*}, y}\left(\alpha_{0}\right) \frac{x_{*}}{\alpha_{0}^{2}}=\frac{x_{*}}{\alpha_{0}}$ shows that $\alpha_{0}=x_{*}$, i.e., each such $x_{*}$ admits only one touching point. Moreover, it follows that $x_{*}=f_{x_{*}, y}\left(x_{*}\right)=\exp (-y-1)=x_{0}$, i.e., such a point $x_{*}$ is unique and coincides with $x_{0}$. Therefore, the number of fixed points of $f_{x, y}$ can change only at $x=x_{0}$. Since $f_{x, y}$ has zero fixed points for sufficiently large $x$, it follows that $f_{x, y}$ has no fixed points for $x>x_{0}$. Moreover, because there is only one touching point at $x_{0}, f_{x, y}$ can have at most two fixed points for $x<x_{0}$. Since $f_{x, y}(\alpha)$ is decreasing in $x$ for each $\alpha$, it follows that $f_{x, y}\left(x_{0}\right)>f_{x_{0}, y}\left(x_{0}\right)=x_{0}$, i.e., the graph of $f_{x, y}$ at $x_{0}$ is above the identity graph. Since the graph of $f_{x, y}$ is below the identity graph at 1 and immediately to the right of 0 , this shows that $f_{x, y}$ has exactly two fixed points for $x \in\left(0, x_{0}\right)$, a fixed point $\alpha_{1}(x)$ in the interval $\left(0, x_{0}\right)$ and a fixed point $\alpha_{2}(x)$ on $\left(x_{0}, 1\right)$.

Finally, since $f_{x, y}$ is continuously decreasing in $x$ for each $\alpha$, it follows that $\alpha_{1}(x)$ is continuously increasing and $\alpha_{2}(x)$ is continuously decreasing in $x$. Continuity and monotonicity imply that $\alpha_{1}(x)$ and $\alpha_{1}(x)$ are differentiable almost everywhere. Taking the derivative of $\alpha_{i}(x)=\exp \left(-\frac{x}{\alpha_{i}(x)}-y\right)$ at a differentiability point yields

$$
\alpha_{i}^{\prime}(x)=\alpha_{i}(x)\left(-\frac{1}{\alpha_{i}(x)}+\frac{x}{\alpha_{i}^{2}(x)} \alpha_{i}^{\prime}(x)\right)=\frac{\alpha_{i}(x)}{x-\alpha_{i}(x)},
$$

where we have used that $\alpha_{1}(x)<x<\alpha_{2}(x)$. Since $\alpha_{i}$ is continuous in $x$, this shows that its derivative is continuous as well and, therefore, $\alpha_{i}$ is differentiable everywhere on $\left(0, x_{0}\right)$.

Proof of Lemma F.1. Fix a vector $p$. Note that $\ell\left(p, \alpha_{p}\right)=\min \left(\frac{1}{\alpha_{p}}(L+w-c-\pi p)^{+}, e\right)$ and hence

$$
\alpha_{p}=\exp \left(-\frac{\gamma}{\alpha_{p}} \sum_{i \in \mathcal{I}}\left(L^{i}+w^{i}-c^{i}-(\pi p)^{i}\right)-\gamma \sum_{i \in \mathcal{D}} e^{i}\right)
$$

Therefore, $\alpha_{p}$ is the largest fixed point of the function $f_{x, y}(\alpha)$ in Lemma F.2 for $y=\gamma \sum_{i \in \mathcal{D}} e^{i}$ and $x=\gamma \sum_{i \in \mathcal{I}}\left(L^{i}+w^{i}-c^{i}-(\pi p)^{i}\right)$. Lemma F.2 thus implies that $\alpha_{p}$ is differentiable in $x$ and $y$, and hence also in $\gamma, L, c, w, e$, and $\pi$ where $\mathcal{D}$ and $\mathcal{I}$ are constant. Continuity follows because $\ell\left(p, \alpha_{p}\right)$ is continuous where $\mathcal{D}$ or $\mathcal{I}$ change.

The construction of the clearing equilibrium as an iterated fixed point of monotonic operators in the proof of Lemma 2.1 yields the following monotonicity properties between asset prices and repayment vectors.

Lemma F.3. Consider a bail-in $(b, s)$ in the financial system $(L, \pi, w, c, e, \beta, \gamma)$.

1. For a fixed clearing payment vector $p$, the asset price $\alpha_{p}$ is non-decreasing in $c^{j}, s^{j}$, and $p^{j}$ for any $j$ and non-increasing in $\gamma, L^{j}, w^{j}, b^{j}$, and $e^{j}$ for any $j$.

2. For a fixed clearing payment vector $p$, for each bank $i, \ell_{p}^{i}$ is non-increasing in $c^{j}$, $s^{j}$, and $p^{j}$ for any $j$ and non-decreasing in $\gamma, L^{j}, w^{j}, b^{j}$, and $e^{j}$ for any $j$ 
3. Let $(\bar{p}, \bar{\ell}, \bar{\alpha})$ be the clearing equilibrium of the financial system. Then $\bar{\alpha}$ and $\bar{p}^{i}$ for any $i$ are non-decreasing in $\beta, c^{j}$, and $s^{j}$ and non-increasing in $\gamma, w^{j}$, and $b^{j}$. Moreover, $\bar{\ell}^{i}$ for any $i$ is non-increasing in $\beta, c^{j}$, and $s^{j}$ and non-decreasing in $\gamma, w^{j}$, and $b^{j}$.

Proof. Fix $p$ and let $\Phi_{p}$ be defined as in the proof of Lemma 2.2. Note that

$$
\Phi_{p}^{i}(x)=\min \left(\exp \left(\gamma \sum_{i=1}^{n} x^{i}\right)\left(L^{i}+w^{i}+b^{i}-c^{i}-s^{i}-(\pi p)^{i}\right)^{+}, e^{i}\right)
$$

is non-increasing in $c^{j}, s^{j}$, and $p^{j}$ for any $j$ and non-decreasing in $\gamma, L^{j}, w^{j}, b^{j}$, and $e^{j}$ for any $j$. The second statement thus follows from a similar application of Lemma B.3 as in the proof of Lemma 2.1. Since $\alpha_{p}$ is a decreasing function of $\sum_{i=1}^{n} \ell_{p}^{i}$, it follows immediately that $\alpha_{p}$ is nondecreasing in $c^{j}, s^{j}$, and $p^{j}$ for any $j$ and non-increasing in $L^{j}, w^{j}, b^{j}$, and $e^{j}$ for any $j$. To show the monotonicty of $\alpha_{p}$ in $\gamma$, observe that $\alpha_{p}$ is differentiable almost everywhere by Lemma F.1. Taking the weak partial derivative in (1) thus yields

$$
\frac{\partial \alpha_{p}}{\partial \gamma}=-\alpha_{p}\left(\sum_{i=1}^{n} \ell_{p}^{i}+\gamma \sum_{i=1}^{n} \frac{\partial \ell_{p}^{i}}{\partial \gamma}\right) \leq 0
$$

This concludes the proof of the first statement. Let now $\Phi$ be defined as in (31). Since $\alpha_{p}$ is non-decreasing in $\beta, c^{j}$ and $s^{j}$ for any $j$ and non-increasing in $\gamma, w^{j}$ and $b^{j}$ for any $j$, it follows immediately that so is $\Phi^{i}(p)$. Another application of Lemma B.3 thus shows that $\bar{p}$ is non-decreasing in $\beta, c^{j}$ and $s^{j}$ for any $j$ and non-increasing in $\gamma, w^{j}$ and $b^{j}$ for any $j$. Therefore, $\bar{\alpha}=\alpha_{\bar{p}}$ is nondecreasing in $\beta, c^{j}$ and $s^{j}$ for any $j$ and non-increasing in $\gamma, w^{j}$ and $b^{j}$ for any $j$. Since $\bar{\ell}=\ell(\bar{p}, \bar{\alpha})$ is a non-increasing function of $\bar{p}$ and $\bar{\alpha}$, the last statement follows.

Lemma F.2 also helps us establishing the existence of the asset price $\alpha_{\mathcal{C}}$. In the following lemma we establish existence, but we defer the remaining statements of Lemma E.2 to a later point.

Lemma F.4. Let $v_{\mathcal{C}}(\alpha)$ denote the right-hand side of 45 . For any set of banks $\mathcal{C}$, (45) has at least one fixed point. Let $\alpha_{\mathcal{C}}$ denote the largest fixed point. For $\alpha \geq \frac{1}{\mathrm{e}}$, there is equivalence between $v_{\mathcal{C}}(\alpha) \leq \alpha$ and $\alpha \geq \alpha_{\mathcal{C}}$.

Proof of Lemma F.4. Fix a set of banks $\mathcal{C}$. We show the existence of a fixed point using a similar argument as in the proof of Lemma 2.2. Define the vector $\varphi(\alpha)$ by setting

$$
\varphi^{i}(\alpha):=\frac{1}{\alpha}\left(L^{i}+w^{i}+\eta^{i}(\alpha, e) 1_{\{i \in \mathcal{C}\}}-c^{i}-s^{i}(\alpha, e)-(\pi L)^{i}\right)^{+} .
$$

It follows from the definitions of $\eta^{i}(\alpha, e)$ and $s^{i}(\alpha, e)$ in (16) and (17), respectively, that $\varphi^{i}$ is nonincreasing in $\alpha$ and that $\varphi^{i}(\alpha) \in\left[0, e^{i}\right]$ for every bank $i$. Thus, $\alpha \circ \varphi$ is a non-decreasing operator that maps $\mathcal{L}:=\times_{i=1}^{n}\left[0, e^{i}\right]$ into itself, where the function $\alpha$ is defined as in (1). By Tarski's fixed point theorem, there exists a lowest fixed point $\ell_{*}$ of $\alpha \circ \varphi$. The greatest fixed point of $v_{\mathcal{C}}$ is thus given by $\alpha_{\mathcal{C}}:=\alpha\left(\ell_{*}\right)$. 
For the second statement, observe that the definitions of $\eta(\alpha, 0)$ and $s(\alpha, 0)$ imply that $v_{\mathcal{C}}(\alpha)=$ $f_{x, y}(\alpha)$ for suitable non-negative $x$ and $y$. Since $v_{\mathcal{C}}(\alpha)=f_{x, y}(\alpha)$ admits a fixed point, it follows from Lemma F.2 that $\alpha_{\mathcal{C}}$ is the unique fixed point on $\left[\frac{1}{e}, 1\right]$. The graph of the function $f_{x, y}$ thus intersects the identity graph precisely once on the interval $\left[\frac{1}{\mathrm{e}}, 1\right]$. Because $f_{x, y}(1) \leq 1$, it follows that $v_{\mathcal{C}}(\alpha) \leq \alpha$ on that interval if and only if $\alpha \geq \alpha_{\mathcal{C}}$

We conclude this section by proving Lemma E.1, which relates the indifference prices for contributions by different rescue consortia.

Proof of Lemma E.1. Since $g$ is strictly convex by Lemma B.4. concavity of $\eta^{i}(\cdot, 0)$ for any bank $i$ implies that $h_{\mathcal{C}}$ is strictly concave. Since $\lim _{\alpha \rightarrow \infty} h_{\mathcal{C}}(\alpha)=-\infty$, it follows that $h_{\mathcal{C}}$ has a unique supremum on $(0, \infty)$. Concavity of $h_{\mathcal{C}}$ implies that it is differentiable almost everywhere. Let $h_{\mathcal{C}}^{\prime}$ denote the weak derivative of $h_{\mathcal{C}}$. Fix any two sets of banks $\mathcal{C} \subseteq \mathcal{C}^{\prime}$. It follows form the definition of $\eta^{i}(\alpha, 0)$ that $h_{\mathcal{C}^{\prime}}^{\prime}(\alpha) \geq h_{\mathcal{C}}^{\prime}(\alpha) \geq-g(\alpha)$. This shows that the supremum of $h_{\mathcal{C}}(\alpha)$ on $(0, \infty)$ is attained at a point $\hat{\alpha}_{\mathcal{C}} \geq \alpha_{\text {ind }}$ and that $\hat{\alpha}_{\mathcal{C}^{\prime}} \geq \hat{\alpha}_{\mathcal{C}}$.

\section{G Proof of Theorem E.3}

In the proof of Theorem E.3, we partially rely on bail-in proposals from the set $\Xi_{*}(\mathcal{C}, \alpha)$. Lemma 3.8 in the main text shows that a complete, feasible, and individually incentive-compatible bail-in $(b, s)$ attains the lower bound on welfare losses in (14) only if $(b, s) \in \Xi_{*}(\mathcal{C}, \alpha)$. In the following result, we show that the converse is true as well.

Lemma G.1. For any set of banks $\mathcal{C}$ and any $\alpha \geq \frac{1}{\mathrm{e}}$, any $(b, s) \in \Xi_{*}(\mathcal{C}, \alpha)$ is a complete, feasible, and individually incentive-compatible bail-in with $\alpha(b, s, 1)=\alpha$ and

$$
W_{\lambda}(b, s, 1)=W_{P}-g\left(\alpha_{P}\right)-h_{\mathcal{C}}(\alpha)
$$

The condition $\alpha \geq \frac{1}{\mathrm{e}}$ is necessary to ensure that the induced asset price indeed coincides with $\alpha$ : Condition (iii) in the Definition E.1 states that the total shortfall in the bail-in $(b, s) \in \Xi_{*}(\mathcal{C}, \alpha)$ is equal to $-\alpha \ln (\alpha) / \gamma$. This pins down the asset price uniquely because the asset price is given by the largest solution to the equation in Condition (iii). However, since $-\alpha \ln (\alpha) / \gamma=x$ has two solutions in general, the attained asset price is equal to $\alpha$ only if $\alpha \geq \frac{1}{\mathrm{e}}$ as stated by the following lemma. This places no restrictions on attainability of complete bail-ins because any such bail-in induces an asset price of at least $\frac{1}{\mathrm{e}}$ by Lemma C.2.

Lemma G.2. The function $f(\alpha)=-\alpha \ln (\alpha) / \gamma$ is strictly concave on $[0,1]$, strictly increasing on $\left[0, \frac{1}{\mathrm{e}}\right]$, strictly decreasing on $\left[\frac{1}{\mathrm{e}}, 1\right]$, and $f\left(\frac{1}{\mathrm{e}}\right)=\frac{1}{\mathrm{e} \gamma}$.

Proof. This follows directly from the the expressions of the first two derivatives

$$
f^{\prime}(\alpha)=-\frac{1+\ln (\alpha)}{\gamma}, \quad f^{\prime \prime}(\alpha)=-\frac{1}{\alpha \gamma} .
$$


Proof of Lemma G.1. Fix $\mathcal{C}, \alpha \geq \frac{1}{\mathrm{e}}$ and a bail-in $(b, s) \in \Xi_{*}(\mathcal{C}, \alpha)$. By Lemma C.1 we may assume without loss of generality that $s^{i}=0$ for $i \in \mathcal{C}$. Define the vector $\ell$ by setting $\ell^{i}=\frac{1}{\alpha}\left(b^{i}-\eta^{i}(\alpha, 0)\right)$ for $i \in \mathcal{C}$ and $\ell^{i}=\frac{1}{\alpha}\left(s^{i}(\alpha, 0)-s^{i}\right)$ for $i \notin \mathcal{C}$. We will show that $(L, \ell, \alpha)$ is a solution to (1), (2), and (3) and hence, it must be the clearing equilibrium of the financial system after transfers $(b, s)$. It follows from the definition of $\ell$ that $b^{i}=\eta^{i}(\alpha, \ell)$ for $i \in \mathcal{C}$ and $s^{i}=s^{i}(\alpha, \ell)$ for $i \notin \mathcal{C}$. The definitions of $\eta(\alpha, \ell)$ and $s(\alpha, \ell)$ in (16) and 17 imply that for every bank $i$,

$$
b^{i}-s^{i} \leq c^{i}+\alpha \ell^{i}+(\pi L)^{i}-L^{i}-w^{i} .
$$

The inequality (48) shows that (3) is satisfied, i.e., every bank is solvent under the clearing payment vector $L$. Since $\min \left(b^{i}, b_{0}^{i}\right)=b^{i}-\eta^{i}(\alpha, 0)$, Lemma 3.8 shows that (2) is satisfied for clearing payment vector $L$ and asset price $\alpha$. Finally, using the definition of $\ell$ together with Condition (iii) in Definition E.1, it is easily seen that (1) is satisfied. Since $\alpha \geq \frac{1}{\mathrm{e}}$, Lemma G.2 shows that $\alpha$ is the largest asset price satisfying (1), hence $(L, \ell, \alpha)$ is the Pareto efficient clearing equilibrium after transfers $(b, s)$. This shows that $(b, s)$ is a complete, feasible bail-in proposal with asset price $\alpha(b, s, 1)=\alpha$. It follows from the definition of $\eta(\alpha, \ell)$ that Condition 2 in Lemma 3.5 is satisfied for every bank $i \in \mathcal{C}$, hence $(b, s)$ is individually incentive-compatible. It now follows from Lemma 3.8 and the definition of $h$ that welfare losses are of the desired form.

Proof of Lemma E.2. Existence of the fixed point has been established in Lemma F.4. Consider the bail-in $(b, s)$ with contributions $b^{i}=\eta^{i}\left(\alpha_{\mathcal{C}}, e\right) 1_{\{i \in \mathcal{C}\}}$ and subsidies $s^{i}=s^{i}\left(\alpha_{\mathcal{C}}, e\right)$. The shortfall of each bank $i$ in the bail-in $(b, s)$ is equal to

$$
\sigma^{i}=\left(L^{i}+w^{i}+\eta^{i}\left(\alpha_{\mathcal{C}}, e\right) 1_{\{i \in \mathcal{C}\}}-c^{i}-s^{i}\left(\alpha_{\mathcal{C}}, e\right)-(\pi L)^{i}\right)^{+} .
$$

Since $\alpha_{\mathcal{C}}$ is the greatest fixed point of (45), $\left(\alpha_{\mathcal{C}}, \frac{\sigma}{\alpha_{\mathcal{C}}}\right)$ is the solution to (1) and (2) for $p=L$ with the largest asset price. The definitions of $\eta(\alpha, \ell)$ and $s(\alpha, \ell)$ in (16) and (17), respectively, imply that $\sigma^{i} \leq \alpha_{\mathcal{C}} e^{i}$ for every bank $i$. Therefore, (3) is satisfied for $p=L, \ell=\frac{\sigma}{\alpha_{\mathcal{C}}}$, and $\alpha=\alpha_{\mathcal{C}}$, showing that $\left(L, \frac{\sigma}{\alpha_{\mathcal{C}}}, \alpha_{\mathcal{C}}\right)$ is a clearing equilibrium. In particular, $(b, s)$ is a complete feasible rescue and hence $\alpha_{\mathcal{C}} \geq \frac{1}{\mathrm{e}}$ by Lemma C.2. It follows from Lemma G.1 that any bail-in from $\Xi_{*}\left(\mathcal{C}, \alpha_{\mathcal{C}}\right)$ attains asset price $\alpha_{\mathcal{C}}$.

For the last statement, let $(b, s)$ be any complete, feasible, and individually incentive-compatible rescue with contributing banks $\mathcal{C}$. It follows from (35) that any bank $i$ liquidates an amount

$$
\ell^{i}(b, s, 1)=\frac{1}{\alpha}\left(\left(s_{0}^{i}-s^{i}\right)^{+}+\left(b^{i}-b_{0}^{i}\right)^{+}\right) .
$$

Since every bank is rescued in $(b, s)$, any bank $i \notin \mathcal{C}$ must receive subsidies of at least $s(\alpha, e)$. Therefore, 49) shows that $\alpha \ell^{i}=\left(s_{0}^{i}-s^{i}\right)^{+} \leq\left(s^{i}(\alpha, 0)-s^{i}(\alpha, e)\right)^{+}=s^{i}(\alpha, 0)-s^{i}(\alpha, e)$. For $i \in \mathcal{C}$, we may assume that $s^{i}=0$ by Lemma C.1. Individual incentive-compatibility implies via 
Condition 2 of Lemma 3.5 that $b^{i} \leq \eta^{i}(\alpha, e)$. For $b_{0}^{i}$ defined as in Lemma 3.8, we obtain

$$
\alpha \ell^{i}=\left(b^{i}-b_{0}^{i}\right)^{+} \leq\left(\eta^{i}(\alpha, e)-b_{0}^{i}\right)^{+}=\eta^{i}(\alpha, e)-\eta^{i}(\alpha, 0) .
$$

It now follows that $\alpha=\exp \left(-\gamma \sum_{i=1}^{n} \ell^{i}\right) \geq v_{\mathcal{C}}(\alpha)$, where the latter function is defined in Lemma F.4. Because $\alpha \geq \frac{1}{\mathrm{e}}$ by Lemma C.2. the result follows from Lemma F.4.

Our next result shows that welfare burning is not needed for sufficiently small rescue consortia because there is no incentive to free-ride.

Lemma G.3. Let $m$ be defined as in Theorem E.3. For any set of banks $\mathcal{C}$ with $|\mathcal{C}| \leq m$ and any $\alpha \geq \alpha_{\mathcal{C}}$, we have $\chi_{\mathcal{C}}(\alpha)=0$.

In the proof, we will rely on the following auxiliary lemma that will be useful also in proofs of other results.

Lemma G.4. Let $(b, s)$ be a complete, feasible, and individually incentive-compatible bail-in with contributing banks $\mathcal{C}$. Let $k=|\mathcal{C}|$ and let $W_{k}$ be defined as in Theorem E.g. Then $W_{\lambda}(b, s, 1) \geq W_{k}$ and $W_{\lambda}(b, s, 1)=W_{k}$ if and only if $(b, s) \in \Xi_{*}\left(\mathcal{C}_{k}, \alpha_{\mathcal{C}_{k}}^{*}\right)$.

Proof. Fix such a bail-in proposal $(b, s)$ with $k$ contributing banks. Lemma E.2 shows that $\alpha_{\mathcal{C}}$ is the smallest price that can be attained in any complete bail-in with contributing banks in $\mathcal{C}$. The maximum of $h_{\mathcal{C}}(\alpha)$ on $\left[\alpha_{\mathcal{C}}, 1\right]$ is thus attained at $\alpha_{\mathcal{C}}^{*}=\max \left(\alpha_{\mathcal{C}}, \min \left(\hat{\alpha}_{\mathcal{C}}, 1\right)\right)$. Therefore,

$$
h_{\mathcal{C}}(\alpha) \leq h_{\mathcal{C}}\left(\alpha_{\mathcal{C}}^{*}\right) \leq h_{\mathcal{C}_{k}}\left(\alpha_{\mathcal{C}_{k}}^{*}\right)
$$

by definition of $\mathcal{C}_{k}$. Together with Lemma 3.9 , this implies that

$$
W_{\lambda}(b, s, 1) \geq W_{P}-g\left(\alpha_{P}\right)-h_{\mathcal{C}}(\alpha) \geq W_{k}
$$

The proof is concluded by observing that the first inequality in 51) holds with equality if and only if $(b, s) \in \Xi_{*}(\mathcal{C}, \alpha)$ and the second inequality (51) binds if and only if $\mathcal{C}=\mathcal{C}_{k}$ and $\alpha=\alpha_{\mathcal{C}_{k}}^{*}$.

Proof of Lemma G.3. Fix a set of banks $\mathcal{C}$ with $|\mathcal{C}| \leq m$ and an asset price $\alpha \geq \alpha_{\mathcal{C}}$. Let $(b, s) \in$ $\Xi_{*}(\mathcal{C}, \alpha)$. By Lemma G.1. $(b, s)$ is a complete, feasible, and individually incentive-compatible bail-in proposal. For any $i \in \mathcal{C}$, let $a_{-i}$ denote the response vector, where every bank but bank $i$ accepts the proposal. Lemmas 3.8 and G.4 imply that

$$
W_{\lambda}\left(b, s, a_{-i}\right)=W_{P}+\left(g\left(\alpha\left(b, s, a_{-i}\right)\right)-g\left(\alpha_{P}\right)\right)-\lambda \sum_{j \in \mathcal{C} \backslash\{i\}} \eta^{j}(\alpha, 0) \geq W_{k-1} \geq W_{N},
$$

where the last inequality follows from the definition of $m$. This shows that no welfare burning is necessary for Condition 1 of Lemma 3.5 to hold. 
Our next result shows that $m$ is well defined, i.e., the sequence $\left(W_{k}\right)_{k \geq 1}$ will decrease below $W_{N}$ eventually. This result is invoked in the proof of Theorem 3.10 .

Lemma G.5. Let $W_{k}$ be defined as in Theorem E.3. There exists $k$ with $W_{k}<W_{N}$. In particular, $m$ in Theorem E.3 is well defined and $(b, s) \in \Xi_{*}\left(\mathcal{C}_{m}, \alpha_{\mathcal{C}_{m}}^{*}\right)$ admits accepting equilibrium $(1, \ldots, 1)$.

Proof. Suppose that $W_{k}>W_{N}$ for every $k \leq n-|\mathcal{F}|-1$. Then there exists no incentive-compatible bail-in with $n-|\mathcal{F}|-1$ contributing banks that attains welfare losses at or below $W_{N}$ by Lemma G.3. We will show that then a bail-in with contributing banks $\mathcal{C}=\mathcal{F}^{c}$ must be incentive compatible and attain welfare losses below $W_{N}$. Consider first the case where $\alpha_{N} \leq \alpha_{\mathcal{C}}$. Define the bail-in $(b, s)$ by setting $b^{i}=\eta^{i}\left(\alpha_{C}, e\right)$ and $s^{i}=s^{i}\left(\alpha_{C}, e\right)$ for every bank $i$. It follows from Lemma G.1 that $(b, s) \in \Xi_{*}\left(\mathcal{C}, \alpha_{\mathcal{C}}\right)$ is a complete, feasible, and individually incentive-compatible bail-in proposal with $\bar{\alpha}(b, s, 1)=\alpha_{\mathcal{C}}$. Since there is no bail-in with $n-|\mathcal{F}|-1$ contributing banks that attains welfare losses below $W_{N}$, Condition 1 in Lemma 3.5 is satisfied. Thus, $a=(1, \ldots, 1)$ is an incentive-compatible response to $(b, s)$ by Lemma 3.5 . It follows from the definition of $\eta$ in (16) that

$$
b^{i}=\eta^{i}\left(\alpha_{\mathcal{C}}, e\right)=\left(c^{i}+\alpha_{\mathcal{C}} e^{i}+(\pi L)^{i}-w^{i}-L^{i}\right)^{+}-V^{i}\left(p_{N}, \alpha_{N}\right)
$$

Since $s^{i}\left(\alpha_{\mathcal{C}}, e\right)=\left(L^{i}+w^{i}-c^{i}-\alpha_{\mathcal{C}} e^{i}-(\pi L)^{i}\right)^{+}$, we deduce that

$$
\begin{aligned}
\sum_{i=1}^{n}\left(b^{i}-s^{i}\right) & =\sum_{i=1}^{n}\left(c^{i}+e^{i}-w^{i}-V^{i}\left(p_{N}, \alpha_{N}\right)\right)-\left(1-\alpha_{\mathcal{C}}\right) \sum_{i=1}^{n} e^{i} \\
& =W_{N}-(1+\lambda) \sum_{i \in \mathcal{D}_{N}} \delta^{i}\left(p_{N}, \alpha_{N}\right)-\left(1-\alpha_{\mathcal{C}}\right) \sum_{i=1}^{n} e^{i} \\
& =\left(\alpha_{\mathcal{C}}-\alpha_{N}\right) \sum_{i=1}^{n} e^{i}+(1-\beta) \sum_{i \in \mathcal{D}_{N}}\left(c^{i}+\alpha_{N} e^{i}+\left(\pi p_{N}\right)^{i}\right)-\sum_{i \in \mathcal{D}_{N}} \delta^{i}\left(p_{N}, \alpha_{N}\right),
\end{aligned}
$$

where we have used Lemma B.1 in the second equation and (6) in the third equation. Using the definitions of welfare losses in the default cascade and in a bail-in, as well as (52), we deduce that

$$
W_{N}-W_{\lambda}(b, s, 1)=(1+\lambda)\left(\left(\alpha_{\mathcal{C}}-\alpha_{N}\right) \sum_{i=1}^{n} e^{i}+(1-\beta) \sum_{i \in \mathcal{D}_{N}}\left(c^{i}+\alpha_{N} e^{i}+\left(\pi p_{N}\right)^{i}\right)\right) \geq 0 .
$$

Consider next the case where $\alpha_{N}>\alpha_{\mathcal{C}}$. Define the bail-in $(b, s)$ by setting $b^{i}=\eta^{i}\left(\alpha_{N}, \ell_{N}\right)$ and $s^{i}=s^{i}\left(\alpha_{N}, \ell_{N}\right)$ for every bank $i$. Since the equity value of a bank is non-decreasing in interbank repayments by Lemma B.2, it follows that $V^{i}(b, s, 1) \geq V^{i}\left(p_{N}, \alpha_{N}\right)$ for every bank $i$. In particular, $(b, s)$ is a complete, feasible, and individually incentive-compatible bail-in. Since $\left(\alpha_{N}, \ell_{N}\right)$ solves (1), it follows that the induced asset price is $\alpha_{N}$. Since there is no bail-in with $n-|\mathcal{F}|-1$ contributing banks that attains welfare losses below $W_{N}$, Condition 1 in Lemma 3.5 is satisfied and hence 
$a=(1, \ldots, 1)$ is an incentive-compatible response to $(b, s)$ by Lemma 3.5 . Observe next that

$$
\alpha\left(e^{i}-\ell_{N}^{i}\right)=\alpha e^{i}-\left(L^{i}+w^{i}-c^{i}-\left(\pi p_{N}\right)^{i}\right)^{+}=V^{i}\left(p_{N}, \alpha_{N}\right)-\left(c^{i}+\left(\pi p_{N}\right)^{i}-w^{i}-L^{i}\right)^{+} .
$$

for any $i \notin \mathcal{D}_{N}$. It follows from the definition of $\eta$ in 16 that

$$
b^{i}=\eta^{i}\left(\alpha_{N}, \ell_{N}\right)=\left(c^{i}+\alpha_{N} \ell_{N}^{i}+(\pi L)^{i}-w^{i}-L^{i}\right)^{+}-\left(V^{i}\left(p_{N}, \alpha_{N}\right)-\alpha_{N}\left(e^{i}-\ell_{N}^{i}\right)\right)^{+} .
$$

Since $s^{i}\left(\alpha_{N}, \ell_{N}\right)=\left(c^{i}+\alpha_{N} \ell_{N}^{i}+(\pi L)^{i}-w^{i}-L^{i}\right)^{-}$, we obtain

$$
\begin{aligned}
\sum_{i=1}^{n}\left(b^{i}-s^{i}\right) & =\sum_{i=1}^{n}\left(c^{i}+\alpha_{N} e^{i}-w^{i}-V^{i}\left(p_{N}, \alpha_{N}\right)\right)-\sum_{i=1}^{n}\left(\alpha_{N}\left(e^{i}-\ell_{N}\right)^{i}-V^{i}\left(p_{N}, \alpha_{N}\right)\right)^{+} \\
& =\sum_{i=1}^{n}\left(c^{i}+e^{i}-w^{i}-V^{i}\left(p_{N}, \alpha_{N}\right)\right)-\left(1-\alpha_{N}\right) \sum_{i=1}^{n} e^{i} .
\end{aligned}
$$

where we have used that $\ell_{N}^{i}=e^{i}$ and $V^{i}\left(p_{N}, \alpha_{N}\right)=0$ for $i \in \mathcal{D}_{N}$ and (53) for $i \notin \mathcal{D}_{N}$ in the second equation. It now follows analogously as above that

$$
W_{N}-W_{\lambda}(b, s, 1)=(1+\lambda)(1-\beta) \sum_{i \in \mathcal{D}_{N}}\left(c^{i}+\alpha_{N} e^{i}+\left(\pi p_{N}\right)^{i}\right) \geq 0
$$

We conclude that in both cases, we have constructed an incentive-compatible bail-in with welfare losses below $W_{N}$. It follows from Lemma G.3 that $W_{n-|\mathcal{F}|} \leq W_{\lambda}(b, s, 1) \leq W_{N}$.

For the last statement, let $(b, s)$ be any bail-in from $\Xi_{*}\left(\mathcal{C}_{m}, \alpha_{\mathcal{C}_{m}}^{*}\right)$. By Lemma G.1. $(b, s)$ is a complete, feasible, and individually incentive-compatible bail-in, that is, Condition 2 of Lemma 3.5 is satisfied for every bank $i \in \mathcal{C}_{m}$. Since $\chi_{\mathcal{C}_{m}}\left(\alpha_{\mathcal{C}_{m}}^{*}\right)=0$ by Lemma G.3, no welfare burning is needed to deter free-riding and hence Condition 1 of Lemma 3.5 is also satisfied for every bank $i \in \mathcal{C}_{m}$. Therefore, $(1, \ldots, 1)$ is an equilibrium response by Lemma 3.5. Since $W_{m}<W_{N}$ by definition, $(1, \ldots, 1)$ is an accepting equilibrium.

Lemma G.6. For any set of banks $\mathcal{C}$ and any $i \in \mathcal{C}$, let $W_{\mathcal{C}}^{i}(\alpha):=W_{P}-g\left(\alpha_{P}\right)-h_{\mathcal{C}}(\alpha)+\chi_{\mathcal{C}}^{i}(\alpha)$. Let $\tilde{\alpha}_{\mathcal{C}}$ be defined as in Theorem E.3. The function $W_{\mathcal{C}}^{\dagger}:=\max _{i \in \mathcal{C}} W_{\mathcal{C}}^{i}$ is quasi-convex with its minimum on $\left[\alpha_{\mathcal{C}}, 1\right]$ attained at $\tilde{\alpha}_{\mathcal{C}}$. Moreover, if $\hat{\chi}_{\mathcal{C}}\left(\tilde{\alpha}_{\mathcal{C}}\right)=0$, then $W_{\mathcal{C}}$ is minimized at $\tilde{\alpha}_{\mathcal{C}}$.

Proof. Fix a set of banks $\mathcal{C}$. For any $i \in \mathcal{C}$, denote $b^{i}(\alpha):=\eta^{i}(\alpha, e)-\eta^{i}(\alpha, 0)$ for the sake of brevity. Set $W_{0}:=W_{N}-W_{P}+g\left(\alpha_{P}\right)$ so that $\chi_{\mathcal{C}}^{i}(\alpha)=\left(W_{0}+h_{\mathcal{C} \backslash\{i\}}(\alpha)-g_{\alpha}\left(b^{i}(\alpha)\right)\right)^{+}$. We begin by computing the partial derivatives of $g_{\alpha}$. Abbreviate $\alpha_{0}(x):=z^{-1}(z(\alpha)+\gamma x)$ so that $g_{\alpha}(x)=g\left(\alpha_{0}(x)\right)-g(\alpha)$. The partial derivatives of $\alpha_{0}(x)$ with respect to $x$ and $\alpha$ are

$$
\alpha_{0}^{\prime}(x)=\frac{\partial \alpha_{0}(x)}{\partial x}=\frac{\gamma}{z^{\prime}\left(\alpha_{0}(x)\right)}, \quad \frac{\partial \alpha_{0}(x)}{\partial \alpha}=\frac{z^{\prime}(\alpha)}{z^{\prime}\left(\alpha_{0}(x)\right)}=\frac{z^{\prime}(\alpha)}{\gamma} \alpha_{0}^{\prime}(x) .
$$

Recalling that $g^{\prime}(\alpha)=\frac{\lambda}{\gamma} z^{\prime}(\alpha)-\sum_{i=1}^{n} e^{i}$ and $z^{\prime}(\alpha)=1+\ln (\alpha)$, as well as $\alpha_{\text {ind }}=\exp \left(\frac{\gamma}{\lambda} \sum_{i=1}^{n} e^{i}-1\right)$, 
the partial derivatives of $g_{\alpha}$ with respect to $x$ and $\alpha$ are equal to

$$
\begin{aligned}
& \frac{\partial g_{\alpha}(x)}{\partial x}=g^{\prime}\left(\alpha_{0}(x)\right) \alpha_{0}^{\prime}(x)=\lambda\left(1-\frac{z^{\prime}\left(\alpha_{\text {ind }}\right)}{z^{\prime}\left(\alpha_{0}(x)\right)}\right) \\
& \frac{\partial g_{\alpha}(x)}{\partial \alpha}=g^{\prime}\left(\alpha_{0}(x)\right) \frac{\partial \alpha_{0}(x)}{\partial \alpha}-g^{\prime}(\alpha)=\sum_{i=1}^{n} e^{i}\left(1-\frac{z^{\prime}(\alpha)}{z^{\prime}\left(\alpha_{0}(x)\right)}\right) .
\end{aligned}
$$

Since $z$ is increasing on $\left[\alpha_{\text {ind }}, 1\right]$, it follows that $\alpha_{0}(x) \geq \alpha \geq \alpha_{\text {ind }}$ for any $\alpha \in\left[\alpha_{\text {ind }}, 1\right]$ and any $x \geq 0$. Convexity of $z$ thus implies that both derivatives in (54) are non-negative. We use this fact to show the following four claims.

Claim.

1. On the interval $\left[\hat{\alpha}_{\mathcal{C}}, 1\right]$, once $\chi_{\mathcal{C}}^{i}(\alpha)$ reaches 0 , it stays 0 .

2. On the interval $\left[\alpha_{\text {ind }}, 1\right], W_{\mathcal{C}}^{i}(\alpha)$ is non-increasing where $\chi_{\mathcal{C}}^{i}(\alpha)>0$.

3. $W_{\mathcal{C}}^{i}$ is non-increasing on $\left[\alpha_{\text {ind }}, \hat{\alpha}_{\mathcal{C}}\right]$.

4. $W_{\mathcal{C}}^{i}$ is non-increasing on $\left[\alpha_{\text {ind }}, \tilde{\alpha}_{\mathcal{C}}^{i}\right]$.

To show the first claim, observe that $h_{\mathcal{C} \backslash\{i\}}$ is non-increasing on $\left[\hat{\alpha}_{\mathcal{C}}, 1\right]$ by Lemma B.4. It is easy to verify from the definition of $\eta^{i}(\alpha, \ell)$ in 16 that $b^{i}(\alpha)$ is non-decreasing in $\alpha$. Therefore,

$$
\frac{\partial\left(W_{0}+h_{\mathcal{C} \backslash\{i\}}(\alpha)-g_{\alpha}\left(b^{i}(\alpha)\right)\right)}{\partial \alpha}=h_{\mathcal{C} \backslash\{i\}}^{\prime}(\alpha)-\frac{\partial g_{\alpha}\left(\bar{b}^{i}\right)}{\partial \alpha}-\frac{\partial g_{\alpha}\left(\bar{b}^{i}\right)}{\partial x} \frac{\partial \bar{b}^{i}}{\partial \alpha}
$$

is non-positive on $\left[\hat{\alpha}_{\mathcal{C}}, 1\right]$. This shows that $\chi_{\mathcal{C}}^{i}(\alpha)$ stays 0 once it reaches 0 on the interval $\left[\hat{\alpha}_{\mathcal{C}}, 1\right]$. For the second claim, observe that for any $\alpha \geq \alpha_{\text {ind }}$, where $\chi_{\mathcal{C}}^{i}(\alpha)$ is positive, (55) implies that

$$
\frac{\partial W_{\mathcal{C}}^{i}(\alpha)}{\partial \alpha}=-\lambda \frac{\partial \eta^{i}(\alpha, 0)}{\partial \alpha}-\frac{\partial g_{\alpha}\left(b^{i}(\alpha)\right)}{\partial \alpha}-\frac{\partial g_{\alpha}\left(b^{i}(\alpha)\right)}{\partial x} \frac{\partial b^{i}(\alpha)}{\partial \alpha} \leq 0 .
$$

To show the third claim, observe first that $W_{P}-g\left(\alpha_{P}\right)-h_{\mathcal{C}}(\alpha)$ is convex and minimized at $\hat{\alpha}_{\mathcal{C}}$ by Lemma E.1. Thus, $W_{\mathcal{C}}^{i}$ is decreasing on the interval $\left[\alpha_{\text {ind }}, \hat{\alpha}_{\mathcal{C}}\right]$ where $\chi_{\mathcal{C}}^{i}(\alpha)=0$. Together with the second claim, this establishes the third claim. If $\tilde{\alpha}_{\mathcal{C}}^{i} \leq \hat{\alpha}_{\mathcal{C}}$, the fourth claim follows from the third. If $\tilde{\alpha}_{\mathcal{C}}^{i}>\hat{\alpha}_{\mathcal{C}}$, then $\chi_{\mathcal{C}}^{i}(\alpha)>0$ for any $\alpha \in\left[\hat{\alpha}_{\mathcal{C}}, \tilde{\alpha}_{\mathcal{C}}^{i}\right]$ by the first claim. The second claim thus implies that $W_{\mathcal{C}}^{i}$ is non-increasing on $\left[\hat{\alpha}_{\mathcal{C}}, \tilde{\alpha}_{\mathcal{C}}^{i}\right]$, which, together with the third claim, establishes the fourth.

Let $\alpha_{\dagger}^{i}:=\max \left(\tilde{\alpha}_{\mathcal{C}}^{i}, \min \left(\hat{\alpha}_{\mathcal{C}}, 1\right)\right)$. The third and fourth claim together imply that $W_{\mathcal{C}}^{i}$ is nonincreasing on the interval $\left[\alpha_{\text {ind }}, \alpha_{\dagger}^{i}\right]$. Since $\chi_{\mathcal{C}}^{i}(\alpha)=0$ for any $\alpha \geq \alpha_{\dagger}^{i}$ by definition of $\tilde{\alpha}_{\mathcal{C}}^{i}$, it follows that $W_{\mathcal{C}}^{i}(\alpha)=W_{P}-g\left(\alpha_{P}\right)-h_{\mathcal{C}}(\alpha)$ on $\left[\alpha_{\dagger}^{i}, 1\right]$. Therefore, $W_{\mathcal{C}}^{i}$ is increasing on $\left[\alpha_{\dagger}^{i}, 1\right]$ by Lemma E.1. This shows that $W_{\mathcal{C}}^{i}$ is quasi-convex and it is minimized at $\alpha_{\dagger}^{i}$.

Let $\alpha^{\dagger}:=\max _{i \in \mathcal{C}} \alpha_{\dagger}^{i}$ and let $i_{0} \in \mathcal{C}$ be such that $\alpha_{\dagger}^{i_{0}}=\alpha^{\dagger}$. Since $\alpha^{\dagger} \geq \tilde{\alpha}_{\mathcal{C}}^{i}$ for any $i \in \mathcal{C}$, it follows that $\chi_{\mathcal{C}}^{i}\left(\alpha^{\dagger}\right)=0$ for any $i \in \mathcal{C}$ and that $W_{\mathcal{C}}^{\dagger}=W_{P}-g\left(\alpha_{P}\right)-h_{\mathcal{C}}(\alpha)$ on the interval $\left[\alpha^{\dagger}, 1\right]$. Since $\alpha^{\dagger} \geq \hat{\alpha}_{\mathcal{C}}$ by construction, $W_{\mathcal{C}}^{\dagger}$ is increasing on $\left[\alpha^{\dagger}, 1\right]$. On the interval $\left[\alpha_{\text {ind }}, \alpha^{\dagger}\right]$, observe that for each $i \in \mathcal{C}$, quasi-convexity of $W_{\mathcal{C}}^{i}$ implies that $W_{\mathcal{C}}^{i}$ is non-increasing on the interval 
$\left\{\alpha \in\left[\alpha_{\text {ind }}, \alpha^{\dagger}\right] \mid W_{\mathcal{C}}^{i}(\alpha) \geq W_{\mathcal{C}}^{i}\left(\alpha^{\dagger}\right)\right\}$. Since $W_{\mathcal{C}}^{\dagger}(\alpha) \geq W_{\mathcal{C}}^{i_{0}}(\alpha) \geq W_{\mathcal{C}}^{i_{0}}\left(\alpha^{\dagger}\right)$ for any $\alpha \in\left[\alpha_{\text {ind }}, \alpha^{\dagger}\right]$, it follows that on the interval $\left[\alpha_{\text {ind }}, \alpha^{\dagger}\right], W_{\mathcal{C}}^{\dagger}$ is non-increasing as the maximum of non-incerasing functions. Therefore, $W_{\mathcal{C}}^{\dagger}$ is quasi-convex and minimized at $\alpha^{\dagger}$. Finally, since $W_{\mathcal{C}}^{\dagger}$ is increasing on $\left[\alpha^{\dagger}, 1\right]$, the minimum of $W_{\mathcal{C}}^{\dagger}$ on $\left[\alpha_{\mathcal{C}}, 1\right]$ is attained at $\max \left(\alpha_{\mathcal{C}}, \alpha^{\dagger}\right)=\tilde{\alpha}_{\mathcal{C}}$. For the last statement, observe that $\hat{\chi}_{\mathcal{C}}\left(\tilde{\alpha}_{\mathcal{C}}\right)=0$ implies $W_{\mathcal{C}}\left(\tilde{\alpha}_{\mathcal{C}}\right)=W_{\mathcal{C}}^{\dagger}\left(\tilde{\alpha}_{\mathcal{C}}\right) \leq W_{\mathcal{C}}^{\dagger}(\alpha) \leq W_{\mathcal{C}}(\alpha)$ for any $\alpha \in\left[\alpha_{\mathcal{C}}, 1\right]$.

Proof of Theorem E.3. By Theorem 3.10, it is enough to show that for any set of banks $\mathcal{C}$, the asset price $\alpha(\mathcal{C})$ minimizes $W_{\mathcal{C}}$ on the interval $\left[\alpha_{\mathcal{C}}, 1\right]$. Suppose first that $|\mathcal{C}| \leq m$. Because $\chi_{\mathcal{C}}(\alpha)=0$ by Lemma G.3 for any $\alpha \geq \alpha_{\mathcal{C}}$, it follows that $W_{\mathcal{C}}(\alpha)=W_{P}-g\left(\alpha_{P}\right)-h_{\mathcal{C}}(\alpha)$, which is minimized at $\alpha_{\mathcal{C}}^{*}$ by Lemma E.1. If $|\mathcal{C}|>m$ and $\hat{\chi}_{\mathcal{C}}\left(\tilde{\alpha}_{\mathcal{C}}\right)=0$, then the statement follows from G.6. In any other case, $W_{\mathcal{C}}$ is minimized at $\alpha_{\mathcal{C}}^{\prime}$ by definition.

Lemma G.6 also gives a direct way of proving Corollary E.4. We separate one fact from the remainder of its proof in the following lemma.

Lemma G.7. $\hat{\chi}_{\mathcal{C}}(1)=\max _{i \in \mathcal{C}} \chi_{\mathcal{C}}^{i}(1)$

Proof. Let $W_{0}:=W_{N}-W_{P}+g\left(\alpha_{P}\right)$. Since $z(1)=0$, it follows that $\hat{\chi}_{\mathcal{C}}(1)=\max _{i \in \mathcal{C}} W_{0}+h_{\mathcal{C} \backslash\{i\}}(1)$. Since $g_{1}(x)=0$, this coincides with $\max _{i \in \mathcal{C}} \chi_{\mathcal{C}}^{i}(1)$.

Proof of Corollary E.4. Fix any set of banks $\mathcal{C}$ and let $\alpha_{\mathcal{C}}^{\prime}$ denote the minimizer of $W_{\mathcal{C}}$. If $\alpha_{\mathcal{C}}^{\prime}=1$, the statement follows from Lemma G.7. If $\alpha_{\mathcal{C}}^{\prime}<1$, then it follows from continuity of $W_{\mathcal{C}}^{\dagger}:=\max _{i \in \mathcal{C}} W_{\mathcal{C}}^{i}$ and $\hat{W}_{\mathcal{C}}(\alpha):=W_{P}-g\left(\alpha_{P}\right)-h_{\mathcal{C}}(\alpha)+\hat{\chi}_{\mathcal{C}}(\alpha)$ that $\alpha_{\mathcal{C}}^{\prime}$ is either a local minimum of $W_{\mathcal{C}}^{\dagger}(\alpha)$, a local minimum of $\hat{W}_{\mathcal{C}}(\alpha)$, or an intersection point of the two functions. In the latter two cases, the statement is immediate. Suppose, therefore, that $\alpha_{\mathcal{C}}^{\prime}$ is a local minimum of $W_{\mathcal{C}}^{\dagger}$. This imposes that $\hat{W}_{\mathcal{C}}\left(\alpha_{\mathcal{C}}^{\prime}\right) \leq W_{\mathcal{C}}^{\dagger}\left(\alpha_{\mathcal{C}}^{\prime}\right)$ and hence $\hat{\chi}\left(\alpha_{\mathcal{C}}^{\prime}\right) \leq \max _{i \in \mathcal{C}} \chi_{\mathcal{C}}^{i}\left(\alpha_{\mathcal{C}}^{\prime}\right)$. By Lemma G.6. $\alpha_{\mathcal{C}}^{\prime}=\tilde{\alpha}_{\mathcal{C}}$ and hence $\hat{\chi}\left(\alpha_{\mathcal{C}}^{\prime}\right) \leq \max _{i \in \mathcal{C}} \chi_{\mathcal{C}}^{i}\left(\alpha_{\mathcal{C}}^{\prime}\right)=0$, showing that $\chi_{\mathcal{C}}\left(\alpha_{\mathcal{C}}^{\prime}\right)=\hat{\chi}_{\mathcal{C}}\left(\alpha_{\mathcal{C}}^{\prime}\right)=0$.

Proof of LemmaE.5. Fix $\alpha \geq \alpha_{\text {ind }}$. Let $\tilde{\chi}_{\mathcal{C}}(\alpha)$ be the unique non-negative solution $\chi$ to

$$
-\frac{\alpha \ln (\alpha)}{\gamma}=\sum_{i \in \mathcal{C}} g_{\alpha}^{-1}\left(\left(W_{N}-W_{P}+g\left(\alpha_{P}\right)+h_{\mathcal{C} \backslash\{i\}}(\alpha)-\chi\right)^{+}\right) 1_{\{\alpha>\alpha(i)\}},
$$

with the convention that $\tilde{\chi}_{\mathcal{C}}(\alpha)=0$ if no non-negative solution to 56 exists. We will first show the following claim, stating that $\tilde{\chi}_{\mathcal{C}}$ and $\left(\chi_{\mathcal{C}}^{i}\right)_{i \in \mathcal{C}}$ contain sufficient information to determine the total amount of welfare burnt.

Claim. For any $\alpha \geq \alpha_{\text {ind }}$, we have $\tilde{\chi}_{\mathcal{C}}(\alpha) \vee \max _{i \in \mathcal{C}} \chi_{\mathcal{C}}^{i}(\alpha)=\hat{\chi}_{\mathcal{C}}(\alpha) \vee \max _{i \in \mathcal{C}} \chi_{\mathcal{C}}^{i}(\alpha)$

It will be convenient to abbreviate $W_{0}:=W_{N}-W_{P}+g\left(\alpha_{P}\right), y_{i}(\alpha):=W_{0}+h_{\mathcal{C} \backslash\{i\}}(\alpha)-\tilde{\chi}_{\mathcal{C}}(\alpha)$, and $\bar{b}^{i}(\alpha):=\eta^{i}(\alpha, e)-\eta^{i}(\alpha, 0)$. With this notation, $\mathcal{C}(\alpha)=\left\{i \in \mathcal{C} \mid y_{i}(\alpha) \geq 0, \alpha \geq \alpha(i)\right\}$ and (56) is equivalent to

$$
-\frac{z(\alpha)}{\gamma}=\sum_{i \in \mathcal{C}(\alpha)} g_{\alpha}^{-1}\left(y_{i}(\alpha)\right)
$$


Since $g_{\alpha}$ is increasing by (54), so is its inverse $g_{\alpha}^{-1}$. Together with $\mathcal{C}(\alpha) \subseteq \mathcal{C}$, this implies that $\tilde{\chi}_{\mathcal{C}}(\alpha) \leq \hat{\chi}_{\mathcal{C}}(\alpha)$ for any $\alpha \geq \alpha_{\text {ind }}$. This immediately shows the claim for any $\alpha$, for which $\hat{\chi}_{\mathcal{C}}(\alpha) \leq$ $\max _{i \in \mathcal{C}} \chi_{\mathcal{C}}^{i}(\alpha)$. Suppose, therefore, that $\hat{\chi}_{\mathcal{C}}(\alpha)>\max _{i \in \mathcal{C}} \chi_{\mathcal{C}}^{i}(\alpha)$. Then, for any $i \in \mathcal{C}$, we have

$$
g_{\alpha}^{-1}\left(\left(W_{0}+h_{\mathcal{C} \backslash\{i\}}(\alpha)-\hat{\chi}_{\mathcal{C}}(\alpha)\right)^{+}\right) \leq g_{\alpha}^{-1}\left(\left(W_{0}+h_{\mathcal{C} \backslash\{i\}}(\alpha)-\chi_{\mathcal{C}}^{i}(\alpha)\right)^{+}\right)=\bar{b}^{i}(\alpha)
$$

Observe that $\alpha(i)$ is defined such that $\bar{b}^{i}(\alpha)>0$ if and only if $\alpha>\alpha(i)$. For any $i$ such that $\alpha \leq \alpha(i)$, it thus follows that $g_{\alpha}^{-1}\left(\left(W_{0}+h_{\mathcal{C} \backslash\{i\}}(\alpha)-\hat{\chi}_{\mathcal{C}}(\alpha)\right)^{+}\right)=0$. Therefore,

$$
g_{\alpha}^{-1}\left(\left(W_{0}+h_{\mathcal{C} \backslash\{i\}}(\alpha)-\hat{\chi}_{\mathcal{C}}(\alpha)\right)^{+}\right)=g_{\alpha}^{-1}\left(\left(W_{0}+h_{\mathcal{C} \backslash\{i\}}(\alpha)-\hat{\chi}_{\mathcal{C}}(\alpha)\right)^{+}\right) 1_{\{\alpha>\alpha(i)\}}
$$

shows that $\hat{\chi}_{\mathcal{C}}(\alpha)$ solves 56 and hence $\hat{\chi}_{\mathcal{C}}(\alpha)=\tilde{\chi}_{\mathcal{C}}(\alpha)$ by uniqueness. This completes the proof of the claim. It also shows that the right-hand side of $(56)$ is a continuous function of $\chi$, which implies continuity of $\tilde{\chi}_{\mathcal{C}}$ as in the proof of Lemma C.6. Moreover, $\tilde{\chi}_{\mathcal{C}}$ is differentiable everywhere except where $\mathcal{C}(\alpha)$ changes size.

We next compute the derivative of $\tilde{\chi}_{\mathcal{C}}-h_{\mathcal{C}}$. For any $\alpha \geq \alpha_{\text {ind }}$, define $\hat{\alpha}_{x}(\alpha)=g^{-1}(x+g(\alpha))$ so that $g_{\alpha}^{-1}(x)=\frac{1}{\gamma}\left(z\left(\hat{\alpha}_{x}(\alpha)\right)-z(\alpha)\right)$. The partial derivatives of $\hat{\alpha}_{x}$ are equal to

$$
\frac{\partial \hat{\alpha}_{x}(\alpha)}{\partial x}=\frac{1}{g^{\prime}\left(g^{-1}(x+g(\alpha))\right)}=\frac{1}{g^{\prime}\left(\hat{\alpha}_{x}(\alpha)\right)}, \quad \frac{\partial \hat{\alpha}_{x}(\alpha)}{\partial \alpha}=\frac{g^{\prime}(\alpha)}{g^{\prime}\left(\hat{\alpha}_{x}(\alpha)\right)} .
$$

Observe that $\alpha \geq \alpha_{\text {ind }}$ implies that $\hat{\alpha}_{x}(\alpha) \geq \alpha \geq \alpha_{\text {ind }}$ and hence $\hat{\alpha}_{x}(\alpha)$ is on the increasing part of $g$. The partial derivatives of $\hat{\alpha}_{x}(\alpha)$ are thus positive. The partial derivatives of $g_{\alpha}^{-1}$ satisfy

$$
\begin{aligned}
& \frac{\partial g_{\alpha}^{-1}(x)}{\partial x}=\frac{1}{\gamma} z^{\prime}\left(\hat{\alpha}_{x}(\alpha)\right) \frac{\partial \hat{\alpha}_{x}(\alpha)}{\partial x}=\frac{z^{\prime}\left(\hat{\alpha}_{x}(\alpha)\right)}{\gamma g^{\prime}\left(\hat{\alpha}_{x}(\alpha)\right)} \geq 0 \\
& \frac{\partial g_{\alpha}^{-1}(x)}{\partial \alpha}=\frac{1}{\gamma} z^{\prime}\left(\hat{\alpha}_{x}(\alpha)\right) \frac{\partial \hat{\alpha}_{x}(\alpha)}{\partial \alpha}-\frac{z^{\prime}(\alpha)}{\gamma}=g^{\prime}(\alpha) \frac{\partial g_{\alpha}^{-1}(x)}{\partial x}-\frac{z^{\prime}(\alpha)}{\gamma} .
\end{aligned}
$$

For any $\alpha \geq \alpha_{\text {ind }}$, for which $\tilde{\chi}_{\mathcal{C}}(\alpha)$ is positive, taking the derivative with respect to $\alpha$ at a differentiability point of $\tilde{\chi}_{\mathcal{C}}(\alpha)$ on both sides in (57) yields

$$
\begin{aligned}
-\frac{z^{\prime}(\alpha)}{\gamma} & =\sum_{i \in \mathcal{C}(\alpha)}\left(\frac{\partial g_{\alpha}^{-1}\left(y_{i}(\alpha)\right)}{\partial \alpha}+\frac{\partial g_{\alpha}^{-1}\left(y_{i}(\alpha)\right)}{\partial x} y_{i}^{\prime}(\alpha)\right) \\
& =\sum_{i \in \mathcal{C}(\alpha)} \frac{\partial g_{\alpha}^{-1}\left(y_{i}(\alpha)\right)}{\partial x}\left(g^{\prime}(\alpha)+y_{i}^{\prime}(\alpha)\right)-|\mathcal{C}(\alpha)| \frac{z^{\prime}(\alpha)}{\gamma}
\end{aligned}
$$

Observe that $\frac{\partial \eta^{i}(\alpha, 0)}{\partial \alpha}=e^{i} 1_{\{\alpha \leq \alpha(i)\}}$ and hence $\frac{\partial \eta^{i}(\alpha, 0)}{\partial \alpha}=0$ for any $i \in \mathcal{C}(\alpha)$. It follows that 
$y_{i}^{\prime}(\alpha)=h_{\mathcal{C} \backslash\{i\}}^{\prime}(\alpha)-\tilde{\chi}_{\mathcal{C}}^{\prime}(\alpha)=h_{\mathcal{C}}^{\prime}(\alpha)-\tilde{\chi}_{\mathcal{C}}^{\prime}(\alpha)$ for any $i \in \mathcal{C}(\alpha)$, hence 59 is equivalent to

$$
\tilde{\chi}_{\mathcal{C}}^{\prime}(\alpha)-h_{\mathcal{C}}^{\prime}(\alpha)=g^{\prime}(\alpha)-\frac{(|\mathcal{C}(\alpha)|-1) \frac{z^{\prime}(\alpha)}{\gamma}}{\sum_{i \in \mathcal{C}(\alpha)} \frac{\partial g_{\alpha}^{-1}\left(y_{i}(\alpha)\right)}{\partial x}}
$$

If $\mathcal{C}(\alpha)$ is a singleton, then this derivative is positive for any $\alpha \geq \alpha_{\text {ind }}$. If $\mathcal{C}(\alpha)$ is not a singleton, observe that for any $\alpha \geq \alpha_{\text {ind }}$, we have

$$
0 \leq \frac{\gamma g^{\prime}(\alpha)}{z^{\prime}(\alpha)}=\lambda-\frac{\gamma \sum_{i=1}^{n} e^{i}}{z^{\prime}(\alpha)} \leq \lambda-\frac{\gamma \sum_{i=1}^{n} e^{i}}{1+\frac{1}{\mathrm{e}}}<\frac{1}{\sqrt{2}},
$$

where we have used (46) in the last inequality. Together with (58), this inequality implies that $\frac{\partial g_{\alpha}^{-1}\left(y_{i}(\alpha)\right)}{\partial x}<\frac{1}{\sqrt{2}}$ for any $i \in \mathcal{C}(\alpha)$. It now follows from 60 that

$$
\tilde{\chi}_{\mathcal{C}}^{\prime}(\alpha)-h_{\mathcal{C}}^{\prime}(\alpha)<\frac{z^{\prime}(\alpha)}{\gamma \sum_{i \in \mathcal{C}(\alpha)} \frac{\partial g_{\alpha}^{-1}\left(y_{i}(\alpha)\right)}{\partial x}}\left(\frac{|\mathcal{C}(\alpha)|}{2}-(|\mathcal{C}(\alpha)|-1)\right) \leq 0
$$

and hence, $y_{i}^{\prime}(\alpha) \geq 0$ wherever $|\mathcal{C}(\alpha)| \geq 2$. As a consequence, $\mathcal{C}(\alpha)$ is non-decreasing on $\left[\alpha_{0}, 1\right]$, where $\alpha_{0}:=\inf \left\{\alpha \in\left[\alpha_{\text {ind }}, 1\right]|| \mathcal{C}(\alpha) \mid \geq 2\right\}$. This, in turn, implies via (61) that $\tilde{\chi}_{\mathcal{C}}(\alpha)-h_{\mathcal{C}}(\alpha)$ is non-increasing on $\left[\alpha_{0}, 1\right]$ and hence so is $\tilde{W}_{\mathcal{C}}$. Since $\mid \mathcal{C}\left(\alpha \mid \leq 1\right.$ on the interval $\left[\alpha_{\text {ind }}, \alpha_{0}\right]$ by definition of $\alpha_{0}, 60$ shows that $\tilde{\chi}_{\mathcal{C}}(\alpha)-h_{\mathcal{C}}(\alpha)$ and hence $\tilde{W}_{\mathcal{C}}$ are non-decreasing on $\left[\alpha_{\text {ind }}, \alpha_{0}\right]$.

To finalize the proof, we distinguish a couple of cases. Consider first the case where $|\mathcal{C}(\alpha)| \geq 2$ for some $\alpha \in\left[\alpha_{\text {ind }}, 1\right]$. Then, since $\mathcal{C}(\alpha)$ is non-decreasing on $\left[\alpha_{0}, 1\right]$, it follows that $|\mathcal{C}(1)| \geq 2$ and hence $\tilde{\chi}_{\mathcal{C}}(1)>0$. This implies that $\max _{i \in \mathcal{C}} \chi_{\mathcal{C}}^{i}(1) \geq \tilde{\chi}_{\mathcal{C}}(1)$ by the above claim and Lemma G.7. It follows that $\tilde{\alpha}_{\mathcal{C}}=1$ by definition. Therefore, $W_{\mathcal{C}}^{\dagger}:=\max _{i \in \mathcal{C}} W_{\mathcal{C}}^{i}$ is non-increasing by Lemma G.6 and hence $W_{\mathcal{C}}(\alpha) \geq W_{\mathcal{C}}^{\dagger}(\alpha) \geq W_{\mathcal{C}}^{\dagger}(1)=W_{\mathcal{C}}(1)$, where we have used that $\max _{i \in \mathcal{C}} \chi_{\mathcal{C}}^{i}(1) \geq \tilde{\chi}_{\mathcal{C}}(1)$ in the last equation. This shows that $W_{\mathcal{C}}$ is minimized at $\alpha=1$.

Suppose next that $|\mathcal{C}(\alpha)|<2$ for all $\alpha \in\left[\alpha_{\text {ind }}, 1\right]$. Then, $\tilde{W}_{\mathcal{C}}$ is increasing by $(60)$. Since $W_{\mathcal{C}}^{\dagger}$ is quasi-convex by Lemma G.6. $W_{\mathcal{C}}=\max \left\{\tilde{W}_{\mathcal{C}}, W_{\mathcal{C}}^{\dagger}\right\}$ is quasi-convex as well. If $\mathcal{C}\left(\tilde{\alpha}_{\mathcal{C}}\right)=\emptyset$, then $\tilde{\chi}_{\mathcal{C}}\left(\tilde{\alpha}_{\mathcal{C}}\right)=0$. The above claim thus implies that $\hat{\chi}_{\mathcal{C}}\left(\tilde{\alpha}_{\mathcal{C}}\right)=0$ and hence $W_{\mathcal{C}}$ is minimized at $\tilde{\alpha_{\mathcal{C}}}$ by Lemma G.6. However, if $\left|\mathcal{C}\left(\tilde{\alpha}_{\mathcal{C}}\right)\right|=1$, then $\tilde{\chi}_{\mathcal{C}}\left(\tilde{\alpha}_{\mathcal{C}}\right)>0$. Therefore, $\tilde{W}_{\mathcal{C}}\left(\tilde{\alpha}_{\mathcal{C}}\right)>W_{\mathcal{C}}^{\dagger}\left(\tilde{\alpha}_{\mathcal{C}}\right)$ and hence the minimum is attained at $\alpha_{\mathcal{C}}^{\prime}<\tilde{\alpha}_{\mathcal{C}}$.

\section{H Comparative Statics Results}

\section{H.1 Complete Bailout}

Many of our comparative statics results will utilize the dependence of $\alpha_{L}$ on the underlying parameters. For the sake of reference, we isolate the partial derivatives of $\alpha_{L}$ in the following lemma. Recall that we denote by $\mathcal{F}$ the set of fundamentally defaulting banks.

Lemma H.1. Let $\xi\left(\gamma, s_{0}, e\right):=1 /\left(1+\ln \left(\alpha_{L}\right)+\gamma \sum_{i \in \mathcal{F}} e^{i}\right)$. Then $\xi\left(\gamma, s_{0}, e\right) \geq 1$. Moreover, $\alpha_{L}$ is 
continuous and where $\mathcal{F}$ is constant, it is differentiable in $\gamma, s_{0}$, and e with partial derivatives

$$
\frac{\partial \alpha_{L}}{\partial \gamma}=\frac{\alpha_{L} \ln \left(\alpha_{L}\right)}{\gamma} \xi, \quad \frac{\partial \alpha_{L}}{\partial s_{0}^{i}}=-\gamma \xi 1_{\{i \notin \mathcal{F}\}}, \quad \frac{\partial \alpha_{L}}{\partial e^{i}}=-\gamma \alpha_{L} \xi 1_{\{i \in \mathcal{F}\}} .
$$

Proof. Continuity and differentiability where $\mathcal{F}$ is constant follows from Lemma F.1 for $p=L$. Since $\ell\left(L, \alpha_{L}\right)=\min \left(\frac{s_{0}}{\alpha_{L}}, e\right)$, we can write $\alpha_{L}=\exp \left(-\gamma \sum_{i \notin \mathcal{F}} \frac{s_{0}^{i}}{\alpha_{L}}-\gamma \sum_{i \in \mathcal{F}} e^{i}\right)$. Implicit differentiation with respect to $\gamma$ at a differentiability point yields

$$
\frac{\partial \alpha_{L}}{\partial \gamma}=\alpha_{L}\left(-\sum_{i \notin \mathcal{F}} \frac{s_{0}^{i}}{\alpha_{L}}-\sum_{i \in \mathcal{F}} e^{i}+\frac{\gamma}{\alpha_{L}^{2}} \sum_{i \notin \mathcal{F}} s_{0}^{i} \frac{\partial \alpha_{L}}{\partial \gamma}\right)=\frac{\alpha_{L} \ln \left(\alpha_{L}\right)}{\gamma}-\left(\ln \left(\alpha_{L}\right)+\gamma \sum_{i \in \mathcal{F}} e^{i}\right) \frac{\partial \alpha_{L}}{\partial \gamma}
$$

where we have used in the second equation that

$$
-\sum_{i \notin \mathcal{F}} \frac{s_{0}^{i}}{\alpha_{L}}-\sum_{i \in \mathcal{F}} e^{i}=\frac{\ln \left(\alpha_{L}\right)}{\gamma} \quad \text { and } \quad \frac{\gamma}{\alpha_{L}} \sum_{i \notin \mathcal{F}} s_{0}^{i}=-\ln \left(\alpha_{L}\right)-\gamma \sum_{i \in \mathcal{F}} e^{i}
$$

Solving (63) for $\frac{\partial \alpha_{L}}{\partial \gamma}$ yields the desired result. For the partial derivatives with respect to $s_{0}^{i}$ and $e^{i}$, observe that $\alpha_{L}$ does not depend on $s_{0}^{i}$ for $i \in \mathcal{F}$ and it does not depend on $e^{i}$ for $i \notin \mathcal{F}$. For $i \notin \mathcal{F}$, using implicit differentiation and the identities in (64), we obtain

$$
\frac{\partial \alpha_{L}}{\partial s_{0}^{i}}=\alpha_{L}\left(-\frac{\gamma}{\alpha_{L}}+\frac{\gamma}{\alpha_{L}^{2}} \sum_{i \notin \mathcal{F}} s_{0}^{i} \frac{\partial \alpha_{L}}{\partial s_{0}^{i}}\right)=-\gamma-\left(\ln \left(\alpha_{L}\right)+\gamma \sum_{i \in \mathcal{F}} e^{i}\right) \frac{\partial \alpha_{L}}{\partial s_{0}^{i}}=-\gamma \xi\left(\gamma, s_{0}, e\right) .
$$

It follows in the same way that for $i \in \mathcal{F}$, the partial derivative with respect to $e^{i}$ is

$$
\frac{\partial \alpha_{L}}{\partial e^{i}}=\alpha_{L}\left(-\gamma+\frac{\gamma}{\alpha_{L}^{2}} \sum_{i \notin \mathcal{F}} s_{0}^{i} \frac{\partial \alpha_{L}}{\partial e^{i}}\right)=-\gamma \alpha_{L}-\left(\ln \left(\alpha_{L}\right)+\gamma \sum_{i \in \mathcal{F}} e^{i}\right) \frac{\partial \alpha_{L}}{\partial e^{i}}=-\gamma \alpha_{L} \xi\left(\gamma, s_{0}, e\right) .
$$

It remains to show that $\xi\left(\gamma, s_{0}, e\right) \geq 1$. It follows from Lemma C.2 that $\alpha_{L} \geq \frac{1}{\mathrm{e}}$ and hence $1+\ln \left(\alpha_{L}\right)+\gamma \sum_{i \in \mathcal{F}} e^{i}>0$, where we have used that $\mathcal{F}$ is non-empty by assumption. The second identity in (64) implies that $1+\ln \left(\alpha_{L}\right)+\gamma \sum_{i \in \mathcal{F}} e^{i} \leq 1$ and, therefore, $\xi\left(\gamma, s_{0}, e\right) \geq 1$.

Lemma H.2. There exist constants $0<\gamma_{1}<\gamma_{2}, 0<\lambda_{1}<\lambda_{2}$ and separating manifolds $0<\mathcal{E}_{1}<\mathcal{E}_{2}$ such that the following conditions hold:

(i) $\alpha_{P}=\alpha_{L}$ for $\gamma \leq \gamma_{1}, \lambda \geq \lambda_{2}$, and $e \leq \mathcal{E}_{1}$,

(ii) $\alpha_{P}=\alpha_{\text {ind }}$ for $\gamma \in\left[\gamma_{1}, \gamma_{2}\right], \lambda \in\left[\lambda_{1}, \lambda_{2}\right]$, and $\mathcal{E}_{1} \leq e \leq \mathcal{E}_{2}$,

(iii) $\alpha_{P}=1$ for $\gamma \geq \gamma_{2}, \lambda \leq \lambda_{1}$, and $e \geq \mathcal{E}_{2}$.

Note there may not be a separating manifold $\mathcal{S}_{1}$ dividing $\alpha_{P}=\alpha_{L}$ and $\alpha_{P}=\min \left(\alpha_{\text {ind }}, 1\right)$. Even though $\alpha_{P}=\alpha_{L}$ for "small" values of $s_{0}^{i}$ and $\alpha_{P}=\min \left(\alpha_{\text {ind }}, 1\right)$ for "large" values of $s_{0}^{i}$, the cutoff may be 0 or infinity depending on what $\alpha_{\text {ind }}$ is. 
Proof. The statement will follow from the monotonicity properties of $\alpha_{L}$ and $\alpha_{\text {ind }}$, and the fact that $\alpha_{P}=\max \left(\min \left(\alpha_{\text {ind }}, 1\right), \alpha_{L}\right)$ by Lemma 3.2. Recall from 10$)$ that $\alpha_{\text {ind }}=\exp \left(\frac{\gamma}{\lambda} E-1\right)$. Let $\lambda_{1}, \gamma_{2}$, and $\mathcal{E}_{2}$ for each $i$ be such that $\alpha_{\text {ind }}=1$. Since $\alpha_{\text {ind }}$ is decreasing in $\lambda$ and increasing in $\gamma$ and $e^{i}$ for every bank $i$, it follows immediately that $\alpha_{P}=1$ for $\lambda \leq \lambda_{1}, \gamma \geq \gamma_{2}$, and $e \geq \mathcal{E}_{2}$.

Observe next that $\alpha_{L} \in\left[\frac{1}{\mathrm{e}}, 1\right)$. Indeed, $\alpha_{L} \geq \frac{1}{\mathrm{e}}$ by Lemma C.2. Moreover, there is at least one fundamentally defaulting bank $i$ by assumption, which liquidates an amount $e^{i}>0$. This implies that $\alpha_{L}<1$ because the price elasticity $\gamma$ is positive by assumption. Therefore, since $\alpha_{L}$ does not depend on $\lambda$, there exists a unique $\lambda_{2}>0$ for which $\alpha_{\text {ind }}=\alpha_{L}$. Moreover, since $\alpha_{\text {ind }}$ is decreasing in $\lambda$, it follows that $\lambda_{2}>\lambda_{1}$ and $\alpha_{P}=\alpha_{\text {ind }}$ for $\lambda \in\left[\lambda_{1}, \lambda_{2}\right]$ and $\alpha_{P}=\alpha_{L}$ for $\lambda \geq \lambda_{2}$.

Since $\alpha_{L}$ is continuous in $\gamma$ and $e$ by Lemma H.1 with $\lim \alpha_{L}=1$ as $\gamma$ or $e$ approach zero, it follows that $\alpha_{L}>\alpha_{\text {ind }}$ for $\gamma$ or $E$ sufficiently small. Since $\alpha_{\text {ind }}$ is increasing and $\alpha_{L}$ is nonincreasing in $\gamma$ and $e^{i}$ for each $i$ by Lemma H.1. there exist unique $\gamma_{1}>0$ and $\mathcal{E}_{1}>0$ where $\alpha_{L}=\alpha_{\text {ind }}$. Because $\alpha_{L}<1$, strict monotonicity of $\alpha_{\text {ind }}$ implies that $\gamma_{1}<\gamma_{2}$ and $\mathcal{E}_{1}<\mathcal{E}_{2}$. Strict monotonicity also implies that $\alpha_{\text {ind }}>\alpha_{L}$ if and only if $\gamma>\gamma_{1}\left(e>\mathcal{E}_{1}\right)$ and hence $\alpha_{P}=\alpha_{L}$ for $\gamma \leq \gamma_{1}\left(e \leq \mathcal{E}_{1}\right)$ and $\alpha_{P}=\alpha_{\text {ind }}$ for $\gamma \in\left[\gamma, \gamma_{2}\right]\left(\mathcal{E}_{1} \leq e \leq \mathcal{E}_{2}\right)$.

Lemma H.3. The asset price $\alpha_{P}$ in the public bailout is non-increasing in $s_{0}^{i}$ for each bank $i$. Moreover, for $\gamma_{1}, \gamma_{2}, \lambda_{1}, \lambda_{2}, \mathcal{E}_{1}, \mathcal{E}_{2}$ as in Lemma H.2, the following statements hold:

(i) For $\gamma \leq \gamma_{1}, \lambda \geq \lambda_{2}$, and $e \leq \mathcal{E}_{1}$, the asset price $\alpha_{P}$ is decreasing in $\gamma$ and $e^{i}$ for $i \in \mathcal{F}$ and it is constant in $\lambda$ and $e^{i}$ for $i \notin \mathcal{F}$.

(ii) For $\gamma \in\left[\gamma_{1}, \gamma_{2}\right], \lambda \in\left[\lambda_{1}, \lambda_{2}\right]$, and $\mathcal{E}_{1} \leq e \leq \mathcal{E}_{2}$, the asset price $\alpha_{P}$ is increasing in $\gamma$ and $e^{i}$ for each $i$ and decreasing in $\lambda$.

Finally, $\alpha_{P}$ is differentiable almost everywhere and $\alpha_{P}>\frac{1}{\mathrm{e}}$.

Proof. Since $\alpha_{\text {ind }}$ does not depend on $s_{0}$, it follows immediately from Lemma H.1 that $\alpha_{P}$ is nonincreasing in $s_{0}^{i}$ for each bank $i$. Statements (i) and (ii) now follow from Lemmas H.1, H.2, and strict monotonicity of $\alpha_{\text {ind }}=\exp \left(\frac{\gamma}{\lambda} E-1\right)$. Because $\alpha_{\text {ind }}$ is strictly increasing in $\gamma$ and $e$ with $\lim \alpha_{\text {ind }}=\frac{1}{\mathrm{e}}$ as $\gamma E$ goes to 0 , it follows from $\mathcal{E}_{1}>0$ and $\gamma_{1}>0$ that $\alpha_{P}>\frac{1}{\mathrm{e}}$. Differentiability almost everywhere follows from differentiability of $\alpha_{\text {ind }}$ and Lemma H.1.

Lemma H.4. The total subsidies $S=\sum_{i=1}^{n} s^{i}$ in a complete bailout are increasing in $s_{0}^{i}$ for each bank $i$. Moreover, for $\gamma_{2}, \lambda_{1}, \lambda_{2}, \mathcal{E}_{1}$, and $\mathcal{E}_{2}$ be as in Lemma H.2, the following statements hold:

(i) For $\lambda \geq \lambda_{2}$ and $e \leq \mathcal{E}_{1}$, the subsidies $S$ are decreasing in $\gamma$ and $e^{i}$ for $i \in \mathcal{F}$ and they are constant in $\lambda$ and $e^{i}$ for $i \notin \mathcal{F}$.

(ii) For $\gamma \in\left[0, \gamma_{2}\right], \lambda \in\left[\lambda_{1}, \lambda_{2}\right]$, and $\mathcal{E}_{1} \leq e \leq \mathcal{E}_{2}$, the subsidies $S$ are increasing in $\gamma$ and $e^{i}$ for each $i$ and decreasing in $\lambda$.

(i) For $\gamma \geq \gamma_{2}, \lambda \leq \lambda_{1}$, and $e \geq \mathcal{E}_{2}$, the subsidies $S$ are constant. 
Proof. For the sake of brevity, denote $S=\sum_{i=1}^{n} s^{i}$. By Lemma 3.2, the total subsidies in a public bailout are equal to

$$
S=S_{0}+\frac{\alpha_{P} \ln \left(\alpha_{P}\right)}{\gamma}
$$

for $\alpha_{P}=\max \left(\min \left(\alpha_{\text {ind }}, 1\right), \alpha_{L}\right)$. Because the total subsidies are differentiable everywhere except where $\alpha_{\text {ind }} \in\left\{\alpha_{L}, 1\right\}$, the total subsidies are weakly differentiable. Taking the (weak) partial derivatives with respect to $\lambda$ and $E$ yields

$$
\frac{\partial S}{\partial \lambda}=\frac{1+\ln \left(\alpha_{P}\right)}{\gamma} \frac{\partial \alpha_{P}}{\partial \lambda}, \quad \frac{\partial S}{\partial E}=\frac{1+\ln \left(\alpha_{P}\right)}{\gamma} \frac{\partial \alpha_{P}}{\partial E}
$$

Since $\alpha_{P}>\frac{1}{\mathrm{e}}$ by Lemma H.3. Equation 66 in conjunction with Lemma H.3 shows the statements for $\lambda$ and $E$. Taking the (weak) partial derivative of (65) with respect to $\gamma$, we obtain

$$
\frac{\partial S}{\partial \gamma}=-\frac{\alpha_{P} \ln \left(\alpha_{P}\right)}{\gamma^{2}}+\frac{1+\ln \left(\alpha_{P}\right)}{\gamma} \frac{\partial \alpha_{P}}{\partial \gamma}
$$

Together with Lemma H.3, this shows that $S$ is strictly increasing for $\gamma \in\left[\gamma_{1}, \gamma_{2}\right]$ and that $S$ is constant for $\gamma \geq \gamma_{2}$. Consider now the case where $\gamma<\gamma_{1}$. Then $\alpha_{P}=\alpha_{L}$ and hence Lemma H.1 implies in conjunction with (67) that

$$
\frac{\partial S}{\partial \gamma}=\frac{\alpha_{L}\left(\ln \left(\alpha_{L}\right)\right)^{2}}{\gamma^{2}} \xi\left(\gamma, s_{0}, e\right)>0
$$

where we have used the fact that $\alpha_{L}<1$. Finally, we compute the (weak) partial derivatives of $S$ with respect to $s_{0}^{i}$. Taking the partial derivative in (65), we obtain

$$
\frac{\partial S}{\partial s_{0}^{i}}=1+\frac{1+\ln \left(\alpha_{P}\right)}{\gamma} \frac{\partial \alpha_{P}}{\partial s_{0}^{i}}
$$

If $\alpha_{P}=\min \left(\alpha_{\text {ind }}, 1\right)$, then $\alpha_{P}$ does not depend on $s_{0}$ and hence $S$ is increasing in $s_{0}^{i}$ for each bank $i$. If $\alpha_{P}=\alpha_{L}$, then we distinguish between where $i$ is a fundamentally defaulting bank or not. If $i \in \mathcal{F}$, then $\alpha_{L}$ does not depend on $s_{0}^{i}$ and hence $S$ is increasing in $s_{0}^{i}$. If $i \notin \mathcal{F}$, Lemma H.1 shows that

$$
\frac{\partial S}{\partial s_{0}^{i}}=1-\left(1+\ln \left(\alpha_{L}\right)\right) \xi\left(\gamma, s_{0}, e\right)=\frac{\gamma \sum_{i \in \mathcal{F}} e^{i}}{1+\ln \left(\alpha_{L}\right)+\gamma \sum_{i \in \mathcal{F}} e^{i}}>0,
$$

were we have used that the set of fundamentally defaulting banks is non-empty.

Lemma H.5. Let $\gamma_{2}, \mathcal{E}_{1}$, and $\mathcal{E}_{2}$ be defined as in Lemma H.2. There exists a separating manifold $0<\mathcal{E}_{0} \leq \mathcal{E}_{1}$ such that the following statements hold:

(i) Welfare losses $W_{P}$ are increasing for $\gamma \leq \gamma_{2}$ and they are constant for $\gamma \geq \gamma_{2}$.

(ii) For $i \in \mathcal{F}$, welfare losses are decreasing in $e^{i}$ for $e \leq \mathcal{E}_{0}$, increasing in $e^{i}$ for $\mathcal{E}_{0} \leq e \leq \mathcal{E}_{2}$, and constant in $e^{i}$ for $e \geq \mathcal{E}_{2}$. 
(iii) For $i \notin \mathcal{F}$, welfare losses are increasing in $e^{i}$ for $e \leq \mathcal{E}_{2}$ and constant in $e^{i}$ for $e \geq \mathcal{E}_{2}$.

Moreover, welfare losses $W_{P}$ in the public bailout are increasing in $\lambda$ and $s_{0}^{i}$ for any bank $i$.

Proof. Lemma 3.2 implies that

$$
W_{P}=\left(1-\alpha_{P}\right) E+\lambda S_{0}+\frac{\lambda \alpha_{P} \ln \left(\alpha_{P}\right)}{\gamma}
$$

for $\alpha_{P}=\max \left(\min \left(\alpha_{\text {ind }}, 1\right), \alpha_{L}\right)$. Welfare losses are thus differentiable everywhere except where $\alpha_{\text {ind }} \in\left\{\alpha_{L}, 1\right\}$. The weak partial derivative of $(69)$ with respect to $\lambda$ is

$$
\frac{\partial W_{P}}{\partial \lambda}=S_{0}+\frac{\alpha_{P} \ln \left(\alpha_{P}\right)}{\gamma}+\frac{\lambda}{\gamma}\left(\ln \left(\alpha_{P}\right)-\ln \left(\alpha_{\text {ind }}\right)\right) \frac{\partial \alpha_{P}}{\partial \lambda}=S_{0}+\frac{\alpha_{P} \ln \left(\alpha_{P}\right)}{\gamma},
$$

where we have used (10) in the first equation and the fact that $\alpha_{P}=\alpha_{\text {ind }}$ for $\lambda \in\left[\lambda_{1}, \lambda_{2}\right]$ and $\frac{\partial \alpha_{P}}{\partial \lambda}=0$ otherwise by Lemmas H.2 and H.3 in the second equation. Together with Lemma 3.2 , this implies that

$$
\frac{\partial W_{P}}{\partial \lambda}=S_{0}+\frac{\alpha_{P} \ln \left(\alpha_{P}\right)}{\gamma} \geq \sum_{i=1}^{n} s_{L}^{i}>0
$$

where we have used in the last inequality that the set of fundamentally defaulting banks is nonempty. Similarly as above, taking the partial derivative in 69 with respect to $\gamma$, we obtain

$$
\frac{\partial W_{P}}{\partial \gamma}=-\frac{\lambda \alpha_{P} \ln \left(\alpha_{P}\right)}{\gamma^{2}}+\frac{\lambda}{\gamma}\left(\ln \left(\alpha_{P}\right)-\ln \left(\alpha_{\text {ind }}\right)\right) \frac{\partial \alpha_{P}}{\partial \gamma} \leq-\frac{\lambda \alpha_{P} \ln \left(\alpha_{P}\right)}{\gamma^{2}},
$$

where we have used that the second term of the middle expression is 0 for $\gamma \geq \gamma_{1}$ by Lemma H.2 and that $\alpha_{P}=\alpha_{L}$ for $\gamma \leq \gamma_{1}$, where $\frac{\partial \alpha_{L}}{\partial \gamma}<0$ by Lemma H.1. This shows that $W_{P}$ is increasing for $\gamma \in\left[\gamma_{1}, \gamma_{2}\right]$ and that $W_{P}$ is constant for $\gamma \geq \gamma_{2}$ as $\alpha_{P}=1$. For $\gamma \leq \gamma_{1}$, where $\alpha_{P}=\alpha_{L}$ by Lemma H.2, Lemma H.1 shows that

$$
\frac{\partial W_{P}}{\partial \gamma}=-\frac{\lambda \alpha_{L} \ln \left(\alpha_{L}\right)}{\gamma^{2}}\left(1-\frac{1+\ln \left(\alpha_{L}\right)-\left(1+\ln \left(\alpha_{\text {ind }}\right)\right)}{1+\ln \left(\alpha_{L}\right)+\gamma \sum_{j \in \mathcal{F}} e^{j}}\right)>0,
$$

where we have a strict inequality since the set of fundamentally defaulting banks is non-empty. For the sensitivity with respect to $s_{0}^{i}$, note first that $\frac{\partial \alpha_{P}}{\partial s_{0}^{i}} \leq 0$ due to Lemma H.1 and the fact that $\alpha_{\text {ind }}$ does not depend on $s_{0}$. Taking the partial derivative of 69 with respect to $s_{0}^{i}$, we obtain

$$
\frac{\partial W_{P}}{\partial s_{0}^{i}}=\lambda+\frac{\lambda}{\gamma}\left(\ln \left(\alpha_{P}\right)-\ln \left(\alpha_{\text {ind }}\right)\right) \frac{\partial \alpha_{P}}{\partial s_{0}^{i}} \leq \lambda
$$

Since $\alpha_{\text {ind }}$ does not depend on $s_{0}$, it follows from Lemma H.1 that $\frac{\partial \alpha_{P}}{\partial s_{0}^{i}}=0$ for $i \in \mathcal{F}$, hence $W_{P}$ is increasing in $s_{0}^{i}$ for such a bank $i$. For $i \notin \mathcal{F}$, we distinguish two cases. If $\alpha_{L} \geq \alpha_{\text {ind }}$, then $\alpha_{P}=\alpha_{L}$. 
Lemma H.1 in conjunction with 72 implies that

$$
\frac{\partial W_{P}}{\partial s_{0}^{i}}=\lambda\left(1-\frac{1+\ln \left(\alpha_{L}\right)-\left(1+\ln \left(\alpha_{\text {ind }}\right)\right)}{1+\ln \left(\alpha_{L}\right)+\gamma \sum_{j \in \mathcal{F}} e^{j}}\right)>0 .
$$

If $\alpha_{L}<\alpha_{\text {ind }}$, then $\alpha_{P}=\min \left(\alpha_{\text {ind }}, 1\right)$ and hence $\frac{\partial \alpha_{P}}{\partial s_{0}^{i}}=0$. Therefore, 72 implies that $W_{P}$ is strictly increasing also in this case. Finally, it follows in the exact same way that

$$
\frac{\partial W_{P}}{\partial e^{i}}=1-\alpha_{P}+\frac{\lambda}{\gamma}\left(\ln \left(\alpha_{P}\right)-\ln \left(\alpha_{\text {ind }}\right)\right) \frac{\partial \alpha_{P}}{\partial e^{i}} \leq 1-\alpha_{P}
$$

with equality if $e \geq \mathcal{E}_{1}$ or $e \leq \mathcal{E}_{1}$ and $i \notin \mathcal{F}$. If $e \leq \mathcal{E}_{1}$ and $i \in \mathcal{F}$, Lemma H.2 implies $\alpha_{P}=\alpha_{L}$. Therefore, Lemma H.1 and (73) show that

$$
\frac{\partial W_{P}}{\partial e^{i}}=\alpha_{L}\left(\frac{1-\alpha_{L}}{\alpha_{L}}-\lambda \frac{1+\ln \left(\alpha_{L}\right)-\left(1+\ln \left(\alpha_{\text {ind }}\right)\right)}{1+\ln \left(\alpha_{L}\right)+\gamma \sum_{j \in \mathcal{F}} e^{j}}\right)=: \alpha_{L}\left(f_{1}\left(\alpha_{L}\right)-f_{2}\left(\alpha_{L}\right)\right)
$$

Function $f_{1}$ is decreasing with $f_{1}(1)=0$. Function $f_{2}$ is increasing with

$$
f_{2}(1)=-\frac{\lambda \ln \left(\alpha_{\text {ind }}\right)}{1+\gamma \sum_{j \in \mathcal{F}} e^{j}}>0
$$

It follows that there exists a unique $\alpha_{L}^{*}<1$ with $f_{1}\left(\alpha_{L}^{*}\right)=f_{2}\left(\alpha_{L}^{*}\right)$ such that $\frac{\partial W_{P}}{\partial e^{i}} \geq 0$ if and only if $\alpha_{L} \leq \alpha_{L}^{*}$. Because $\alpha_{L}$ is decreasing in $e^{i}$ by Lemma H.1, there exists a separating manifold $\mathcal{E}_{*}$ such that $\alpha_{L} \leq \alpha_{L}^{*}$ if and only if $e \geq \mathcal{E}_{*}$. The statement thus follows for $\mathcal{E}_{0}=\mathcal{E}_{*} \wedge \mathcal{E}_{1}$.

Proof of Lemma 4.1. The statement follows from Lemmas H.3, H.4, and H.5.

\section{H.2 Default Cascade}

It will be convenient to denote by $\mathcal{D}_{N}:=\mathcal{D}\left(p_{N}, \ell_{N}, \alpha_{N}\right)$ the set of defaulting banks in absence of intervention, by $\mathcal{C}_{N}:=\left\{i \in \mathcal{D}_{N} \mid \delta^{i}\left(p_{N}, \alpha_{N}\right)=0\right\}$, the set of defaulting banks which are able to repay a positive amount to their junior creditors, by $\mathcal{S}_{N}=\mathcal{D}_{N}^{c}$ the set of solvent banks, and by $\mathcal{I}_{N}:=\left\{i \mid 0<\ell_{N}^{i}<e^{i}\right\}$ the set of solvent but illiquid banks. Before we get into the sensitivity analysis, we address continuity and differentiability of the default cascade.

Lemma H.6. $p_{N}$ and $\alpha_{N}$ are differentiable in $L, \pi, c, w, e, \beta$, and $\gamma$ where $\mathcal{D}_{N}, \mathcal{C}_{N}$, and $\mathcal{I}_{N}$ are constant. Moreover, $p_{N}$ and $\alpha_{N}$ are continuous where $\mathcal{D}_{N}$ is constant. Finally, $p_{N}$ is differentiable in $\alpha_{N}$ where $\mathcal{D}_{N}, \mathcal{C}_{N}$, and $\mathcal{I}_{N}$ are constant.

Proof. Observe that we can write $p_{N}$ in vector form as $p_{N}^{\mathcal{S}_{N}}=L^{\mathcal{S}_{N}}, p_{N}^{\mathcal{D}_{N} \backslash \mathcal{C}_{N}}=0$, and

$$
p_{N}^{\mathcal{C}_{N}}=\left(I-\beta \pi^{\mathcal{C}_{N}, \mathcal{C}_{N}}\right)^{-1}\left(\beta c^{\mathcal{C}_{N}}+\beta \alpha_{p_{N}} e^{\mathcal{C}_{N}}+\beta \pi^{\mathcal{C}_{N}, \mathcal{S}_{N}} L^{\mathcal{S}_{N}}-w^{\mathcal{C}_{N}}\right),
$$

where we use the subvector and submatrix notation introduced in Lemma H.9. Since $\alpha_{p}$ is dif- 
ferentiable in $p$ by Lemma F.1, this shows differentiability of $p_{N}$ where $\mathcal{D}_{N}, \mathcal{C}_{N}, \mathcal{S}_{N}$, and $\mathcal{I}_{N}$ are constant. Then, differentiability of $\alpha_{N}=\alpha_{p_{N}}$ follows from Lemma F.1. It follows from (2) and (3) that $p_{N}$ and $\alpha_{N}$ are continuous unless the set $\mathcal{D}_{N}$ of defaulting banks changes.

Lemma H.7. For each $i$, there exists $e_{*}^{i} \geq 0,0 \leq c_{1}^{i} \leq c_{*}^{i} \leq c_{2}^{i}$, and $0 \leq w_{1}^{i} \leq w_{*}^{i} \leq w_{2}^{i}$ such that $i$ defaults for $e^{i}<e_{*}^{i}, c^{i}<c_{*}^{i}$, or $w^{i}>w_{*}^{i}$ and it is solvent for $e^{i} \geq e_{*}^{i}, c^{i} \geq c_{*}^{i}$, or $w^{i} \leq w_{*}^{i}$. Moreover, bank $i$ is in $\mathcal{D}_{N} \backslash \mathcal{C}_{N}$ for $c^{i} \leq c_{1}^{i}$ or $w^{i} \geq w_{2}^{i}$, and bank $i$ is in $\mathcal{S}_{N} \backslash \mathcal{I}_{N}$ for $c^{i} \geq c_{2}^{i}$ or $w^{i} \leq w_{1}^{i}$.

Proof. Observe first that a bank $i$ is solvent if and only if $L^{i}+w^{i} \leq c^{i}+\alpha_{N} e^{i}+\left(\pi p_{N}\right)^{i}$. Since $\alpha_{N}$ and $p_{N}$ do not depend on the size of the illiquid asset held by a solvent bank, it follows that if $i$ is solvent for some $e_{*}^{i}$, it is solvent for all $e^{i}>e_{*}^{i}$. This establishes the cut-off form of bank $i$ 's solvency. Moreover, the cutoff is finite because $\alpha_{N}>0$. Since $\alpha_{N}$ and $p_{N}$ are non-decreasing in $c^{i}$ and non-increasing in $w^{i}$ by Statement 3 of Lemma F.3 it follows that there exist cutoffs $c_{*}^{i}$ and $w_{*}^{i}$ such that bank $i$ is solvent if and only if $c^{i} \geq c_{*}^{i}$ and $w^{i} \leq w_{*}^{i}$, respectively.

For the second statement, observe that $i \in \mathcal{D}_{N} \backslash \mathcal{C}_{N}$ if and only if $w^{i}-\beta\left(c^{i}+\alpha_{N} e^{i}+\left(\pi p_{N}\right)^{i}\right)>0$ and that $i \in \mathcal{S}_{N} \backslash \mathcal{I}_{N}$ if and only if $\ell_{N}^{i}=0$. Since for a bank in $\mathcal{D}_{N} \backslash \mathcal{C}_{N}$, a change in $c^{i}$ or $w^{i}$ does not affect the asset price or the vector of repayments, it follows that if a bank $i$ is in $\mathcal{D}_{N} \backslash \mathcal{C}_{N}$ for some $c_{1}^{i}$ (some $w_{2}^{i}$ ), then it is also in $\mathcal{D}_{N} \backslash \mathcal{C}_{N}$ for $c^{i} \leq c_{1}^{i}$ (for $w^{i} \geq w_{2}^{i}$ ). Since $\mathcal{D}_{N} \backslash \mathcal{C}_{N} \subseteq \mathcal{D}_{N}$, it follows that $c_{1}^{i} \leq c_{*}^{i}\left(w_{2}^{i} \geq w_{*}^{i}\right)$. Similarly, for a bank in $\mathcal{S}_{N} \backslash \mathcal{I}_{N}$, a change in $c^{i}$ or $w^{i}$ does not affect the asset price or the vector of repayments, hence if a bank $i$ is in $\mathcal{S}_{N} \backslash \mathcal{I}_{N}$ for some $c_{2}^{i}$ (some $w_{1}^{i}$ ), then it is also in $\mathcal{S}_{N} \backslash \mathcal{I}_{N}$ for $c^{i} \geq c_{2}^{i}$ (for $\left.w^{i} \leq w_{1}^{i}\right)$. Again, it follows that $c_{*}^{i} \leq c_{2}^{i}\left(w_{1}^{i} \leq w_{*}^{i}\right)$ by monotonicity. Finally, as $c^{i}$ goes to infinity, bank $i$ will not have to liquidate anything, hence $i \in \mathcal{S}_{N} \backslash \mathcal{I}_{N}$, showing that $c_{2}^{i}$ is finite. As $w^{i}$ goes to infinity, bank $i$ is not able to repay anything to its junior creditors and hence $i \in \mathcal{D}_{N} \backslash \mathcal{C}_{N}$, showing that $w_{2}^{i}$ is finite.

Lemma H.8. For each $i$, let $e_{*}^{i}, c_{1}^{i}, c_{*}^{i}, c_{2}^{i}, w_{1}^{i}, w_{*}^{i}$, and $w_{2}^{i}$ be as in Lemma H.7. Then $\alpha_{N}$ is decreasing for $e<e_{*}^{i}, \alpha_{N}$ has a positive discontinuity at $e_{*}^{i}$, and $\alpha_{N}$ is constant for $e^{i}>e_{*}^{i}$. The asset price $\alpha_{N}$ is constant for $c^{i} \leq c_{1}^{i}$ and $c^{i} \geq c_{2}^{i}$, increasing for $c^{i} \in\left[c_{*}^{i}, c_{2}^{i}\right]$, non-decreasing for $c^{i} \in\left[c_{1}^{i}, c_{*}^{i}\right]$ with strict monotonicity if $\theta_{\mathcal{I}_{N}}^{i}(\beta, \pi)>0$. The asset price $\alpha_{N}$ is decreasing in $\gamma$, it is non-decreasing in $\beta$, and constant in $\lambda$. Moreover, $\alpha_{N}$ is locally increasing in $\beta$ if and only if at least one bank in $\mathcal{C}_{N}$ has a creditor in $\mathcal{I}_{N}$.

In order to prove Lemma $\mathrm{H} .8$, we require the following two auxiliary results. The first implies that $\theta_{\mathcal{S}}^{i}(\beta, \pi) \leq 1$ for every $i \in \mathcal{C}_{N}$ and any set $\mathcal{S}$ by setting $y^{\mathcal{C}}=\rho_{i}^{\mathcal{C}_{N}}$.

Lemma H.9. For any two sets of banks $\mathcal{I}$ and $\mathcal{C}$ and any vector $y$, let $y^{\mathcal{C}}$ denote the subvector with entries in $\mathcal{C}$ and let $\pi^{\mathcal{I}, \mathcal{C}}$ denote the submatrix of $\pi$ with rows in $\mathcal{I}$ and columns in $\mathcal{C}$. If $y^{\mathcal{C}}$ is non-negative and $\mathcal{I}$ and $\mathcal{C}$ are disjoint, we have

$$
\sum_{i \in \mathcal{I}} \pi^{i, \mathcal{C}}\left(I-\beta \pi^{\mathcal{C}, \mathcal{C}}\right)^{-1} y^{\mathcal{C}} \leq \sum_{i \in \mathcal{C}} y^{i}
$$


Proof. Let $x^{\mathcal{C}}:=\left(I-\beta \pi^{\mathcal{C}, \mathcal{C}}\right)^{-1} y^{\mathcal{C}}$. We can expand $x^{\mathcal{C}}$ using a power series to $x^{\mathcal{C}}=\sum_{k=0}^{\infty}\left(\beta \pi^{\mathcal{C}, \mathcal{C}}\right)^{k} y^{\mathcal{C}}$. Since every entry of $y$ and $\pi$ is non-negative, it follows that $x^{i} \geq y^{i} \geq 0$. Let $\mathbf{1}_{\mathcal{C}} \in \mathbb{R}^{|\mathcal{C}|}$ denote the vector with ones in every component. Then, we can write

$$
\beta \sum_{i, j \in \mathcal{C}} \pi^{i j} x^{j}=\beta \mathbf{1}_{\mathcal{C}}^{\top} \pi^{\mathcal{C}, \mathcal{C}} x^{\mathcal{C}}+\mathbf{1}_{\mathcal{C}}^{\top} x^{\mathcal{C}}-\mathbf{1}_{\mathcal{C}}^{\top} x^{\mathcal{C}}=\mathbf{1}_{\mathcal{C}}^{\top} x^{\mathcal{C}}-\mathbf{1}_{\mathcal{C}}^{\top}\left(I-\beta \pi^{\mathcal{C}, \mathcal{C}}\right) x^{\mathcal{C}}=\sum_{i \in \mathcal{C}}\left(x^{i}-y^{i}\right)
$$

Since $\mathcal{I}$ and $\mathcal{C}$ are disjoint and $\pi$ is column-stochastic, we obtain

$$
\beta \sum_{i \in \mathcal{I}} \pi^{i, \mathcal{C}} x^{\mathcal{C}}=\beta \sum_{j \in \mathcal{C}} x^{j} \sum_{i \in \mathcal{I}} \pi^{i j} \leq \beta \sum_{j \in \mathcal{C}} x^{j}\left(1-\sum_{i \in \mathcal{C}} \pi^{i j}\right)=\sum_{i \in \mathcal{C}}\left(y^{i}-(1-\beta) x^{i}\right) \leq \beta \sum_{i \in \mathcal{C}} y^{i},
$$

where we have used (75) in the penultimate equation and $y^{i} \geq x^{i}$ in the last equation.

Lemma H.10. At any differentiability point of any financial system, the quantity

$$
\chi_{N}:=\frac{\gamma}{\alpha_{N}} \sum_{i \in \mathcal{I}_{N}}\left(L^{i}+w^{i}-c^{i}-\left(\pi p_{N}\right)^{i}\right)+\gamma \sum_{i \in \mathcal{I}_{N}} \frac{\partial\left(\pi p_{N}\right)^{i}}{\partial \alpha_{N}}
$$

is strictly smaller than 1.

Proof. Because there is at least one fundamentally defaulting bank by assumption, $\sum_{i=1}^{n} \ell_{p}^{i}>0$ for any vector of repayments $p$. Therefore, 47 implies that $\frac{\partial \alpha_{p}}{\partial \gamma}<0$. Since $\alpha_{p}$ is non-decreasing in $p$ and $p_{N}$ is non-increasing in $\gamma$ by Lemma F.3 it follows from total differentiation that

$$
\frac{\partial \alpha_{N}}{\partial \gamma}=\frac{\partial \alpha_{p}}{\partial p} \frac{\partial p_{N}}{\partial \gamma}+\frac{\partial \alpha_{p}}{\partial \gamma}<0
$$

Observe that we can write

$$
\alpha_{N}=\exp \left(-\frac{\gamma}{\alpha_{N}} \sum_{i \in \mathcal{I}_{N}}\left(L^{i}+w^{i}-c^{i}-\left(\pi p_{N}\right)^{i}\right)-\gamma \sum_{i \in \mathcal{D}_{N}} e^{i}\right)
$$

Implicitly differentiating (78) with respect to $\gamma$, we obtain

$$
\frac{\partial \alpha_{N}}{\partial \gamma}=\frac{\alpha_{N} \ln \left(\alpha_{N}\right)}{\gamma}+\chi_{N} \frac{\partial \alpha_{N}}{\partial \gamma}=\frac{\alpha_{N} \ln \left(\alpha_{N}\right)}{\gamma\left(1-\chi_{N}\right)} .
$$

By (77), this term has to be negative, implying that $\chi_{N}<1$.

Proof of Lemma H.8. Since $\lambda$ is just a welfare parameter, it is clear that $\alpha_{N}$ is constant in $\lambda$. It follows from (77) that $\alpha_{N}$ is strictly decreasing in $\gamma$ at continuity points and it follows from Statement 3 of Lemma F.3 that $\alpha_{N}$ is decreasing in $\gamma$ at discontinuities. Statement 3 of Lemma F.3 shows that $\alpha_{N}$ is non-decreasing in $\beta$.

Let us now characterize when $\alpha_{N}$ is strictly increasing in $\beta$. Fix a bank $i \in \mathcal{I}_{N}$ and observe 
that $\ell_{N}^{i}=\frac{1}{\alpha_{N}}\left(L^{i}+w^{i}-c^{i}-\left(\pi p_{N}\right)^{i}\right)$. Taking the partial derivative with respect to $\beta$ yields

$$
\frac{\partial \ell_{N}^{i}}{\partial \beta}=-\frac{1}{\alpha_{N}} \ell_{N}^{i} \frac{\partial \alpha_{N}}{\partial \beta}-\frac{1}{\alpha_{N}} \sum_{j \in \mathcal{C}_{N}} \pi^{i j} \frac{\partial p_{N}^{j}}{\partial \beta}
$$

where we have used the fact that $p_{N}^{i}$ is constant for solvent banks and banks in $\mathcal{D}_{N} \backslash \mathcal{C}_{N}$. Since $\ell_{N}^{j}$ is constant for $j \notin \mathcal{I}_{N}$, multiplying $(79)$ by $-\gamma \alpha_{N}$ and summing over all banks yields

$$
\frac{\partial \alpha_{N}}{\partial \beta}=-\gamma \alpha_{N} \sum_{i \in \mathcal{I}_{N}} \frac{\partial \ell_{N}^{i}}{\partial \beta}=\gamma \sum_{i \in \mathcal{I}_{N}} \ell_{N}^{i} \frac{\partial \alpha_{N}}{\partial \beta}+\gamma \sum_{i \in \mathcal{I}_{N}} \sum_{j \in \mathcal{C}_{N}} \pi^{i j} \frac{\partial p_{N}^{j}}{\partial \beta}=\frac{\gamma \sum_{i \in \mathcal{I}_{N}} \sum_{j \in \mathcal{C}_{N}} \pi^{i j} \frac{\partial p_{N}^{j}}{\partial \beta}}{1-\gamma \sum_{i \in \mathcal{I}_{N}} \ell_{N}^{i}} .
$$

By Statement 3 of Lemma F.3, this quantity is non-negative and it is different from 0 if and only if there exists a bank $j \in \mathcal{C}_{N}$ with a creditor $i \in \mathcal{I}_{N}$.

For the sensitivity with respect to $e^{i}$ and $c^{i}$, we first determine the change in $\pi p_{N}$ in the asset price $\alpha_{N}$. Let $\mathcal{S}$ be any set of banks with $\mathcal{S} \cap \mathcal{C}_{N}=\emptyset$. Using the subvector and submatrix notation introduced in Lemma H.9, it follows from (74) that

$$
\sum_{j \in \mathcal{S}} \frac{\partial\left(\pi p_{N}\right)^{j}}{\partial \alpha_{N}}=\beta \mathbf{1}_{\mathcal{S}}^{\top} \pi^{\mathcal{S}, \mathcal{C}_{N}}\left(I-\beta \pi^{\mathcal{C}_{N}, \mathcal{C}_{N}}\right)^{-1} e^{\mathcal{C}_{N}}=\sum_{j \in \mathcal{C}_{N}}\left(\beta \theta_{\mathcal{S} \backslash \mathcal{D}_{N}}^{j}+\theta_{\mathcal{S} \cap \mathcal{D}_{N} \backslash \mathcal{C}_{N}}^{j}\right) e^{j} \geq 0
$$

at any differentiability point. Taking the partial derivative of (74) with respect to $e^{i}$ yields

$$
\sum_{j \in \mathcal{S}} \frac{\partial\left(\pi p_{N}\right)^{j}}{\partial e^{i}}=\sum_{j \in \mathcal{S}} \frac{\partial\left(\pi p_{N}\right)^{j}}{\partial \alpha_{N}} \frac{\partial \alpha_{N}}{\partial e^{i}}+\alpha_{N}\left(\beta \theta_{\mathcal{S} \backslash \mathcal{D}_{N}}^{i}(\beta, \pi)+\theta_{\mathcal{S} \cap \mathcal{D}_{N} \backslash \mathcal{C}_{N}}(\beta, \pi)\right) 1_{\left\{i \in \mathcal{C}_{N}\right\}}
$$

Using (81) for $\mathcal{S}=\mathcal{I}_{N}$, implicit differentiation in $(78)$ with respect to $e^{i}$ yields

$$
\frac{\partial \alpha_{N}}{\partial e^{i}}=\chi_{N} \frac{\partial \alpha_{N}}{\partial e^{i}}-\gamma \alpha_{N} 1_{\left\{i \in \mathcal{D}_{N}\right\}}+\gamma \alpha_{N}^{2} \beta \theta_{\mathcal{I}_{N}}^{i}(\beta, \pi) 1_{\left\{i \in \mathcal{C}_{N}\right\}} .
$$

For $i \in \mathcal{C}_{N}$, solving 82 for $\frac{\partial \alpha_{N}}{\partial e^{i}}$ and using the bound in Lemma H.9, we obtain

$$
\frac{\partial \alpha_{N}}{\partial e^{i}} \leq \frac{\gamma \alpha_{N}\left(\beta \alpha_{N}-1\right)}{1-\chi_{N}}<0
$$

where we have used that $\chi_{N}<1$ by Lemma H.10. It follows in the same way that the partial derivative in 82 is negative for $i \in \mathcal{D}_{N} \backslash \mathcal{C}_{N}$ and 0 for $i \notin \mathcal{D}_{N}$. For discontinuity points, observe that Lemma H.7 shows that crossing $e_{*}^{i}$ reduces bankruptcy costs, hence $p_{N}$ and $\alpha_{N}$ have an upward discontinuity. All other discontinuities occur when an increase in $e^{i}$ decreases the price to a level, at which another bank becomes insolvent, causing a downward discontinuity in $p_{N}$ and $\alpha_{N}$.

For the sensitivity with respect to $c^{i}$, observe first that at continuity points, (74) implies that 
the weak partial derivative of $\left(\pi p_{N}\right)^{i}$ with respect to $c^{i}$ equals

$$
\sum_{j \in \mathcal{I}_{N}} \frac{\partial\left(\pi p_{N}\right)^{j}}{\partial c^{i}}=\sum_{j \in \mathcal{I}_{N}} \frac{\partial\left(\pi p_{N}\right)^{j}}{\partial \alpha_{N}} \frac{\partial \alpha_{N}}{\partial c^{i}}+\beta \theta_{\mathcal{I}_{N}}^{i}(\beta, \pi) 1_{\left\{i \in \mathcal{C}_{N}\right\}}
$$

Using (83), implicit differentiation in $(78)$ with respect to $c^{i}$ yields

$$
\frac{\partial \alpha_{N}}{\partial c^{i}}=\gamma 1_{\left\{i \in \mathcal{I}_{N}\right\}}+\chi_{N} \frac{\partial \alpha_{N}}{\partial c^{i}}+\gamma \beta \theta_{\mathcal{I}_{N}}^{i}(\beta, \pi) 1_{\left\{i \in \mathcal{C}_{N}\right\}} .
$$

Consider first the case where $c^{i} \leq c_{1}^{i}$ or $c^{i} \geq c_{2}^{i}$. Then $i \notin \mathcal{I}_{N} \cup \mathcal{C}_{N}$ by Lemma H.7, hence it follows from (84) that $\alpha_{N}$ is constant in $c^{i}$. If $c^{i} \in\left[c_{1}^{i}, c_{*}^{i}\right]$, then $i \in \mathcal{I}_{N}$ by Lemma H.7. Solving (84) for $\frac{\alpha_{N}}{\partial c^{i}}$ shows that $\alpha_{N}$ is increasing in $c^{i}$ by Lemma H.10. Finally, if $c^{i} \in\left[c_{*}^{i}, c_{2}^{i}\right]$, then $i \in \mathcal{C}_{N}$ by Lemma H.7. It follows from (84) $\alpha_{N}$ is increasing in $c^{i}$ at continuity points if $\theta_{\mathcal{I}_{N}}^{i}(\beta, \pi)$ is positive and $\alpha_{N}$ is constant otherwise. Statement 3 of Lemma F.3 shows that $\alpha_{N}$ is increasing in $c^{i}$ at discontinuities. The statement now follows from Lemma H.7.

Let $\pi^{\mathcal{C}, \mathcal{C}}$ denote the submatrix of entries corresponding only to banks in $\mathcal{C}_{N}$ and define $\bar{\pi}:=$ $\left(I-\beta \pi^{\mathcal{C}, \mathcal{C}}\right)^{-1}$. Note that $\bar{\pi}=\sum_{k=0}^{\infty}\left(\beta \pi^{\mathcal{C}, \mathcal{C}}\right)^{k}$ captures the direct exposure of banks in $\mathcal{C}_{N}$ towards each other as well as the higher-order exposures.

Lemma H.11. Fix a bank $i \in \mathcal{C}_{N}$. Interbank repayments $p_{N}^{i}$ are decreasing in $\gamma$ and $w^{i}$, they are increasing in $\beta$ and $c^{i}$, and they are constant in $\lambda$ and $e^{j}$ for $j \in \mathcal{S}_{N}$. Moreover, at continuity points, $p_{N}^{i}$ is decreasing in $e^{j}$ for $j \in \mathcal{D}_{N} \backslash \mathcal{C}_{N}$ and it is increasing in $e^{j}$ for $j \in \mathcal{C}_{N}$ if and only if

$$
\frac{\bar{\pi}^{i j}}{\sum_{k \in \mathcal{C}_{N}} \bar{\pi}^{i k} e^{k}}>-\frac{1}{\alpha_{N}} \frac{\partial \alpha_{N}}{\partial e^{j}}
$$

Proof. Fix a bank $i \in \mathcal{C}_{N}$ and write $p_{N}^{i}=\beta\left(c^{i}+\alpha_{N} e^{i}+\left(\pi p_{N}\right)^{i}\right)-w^{i}$. Since $p_{N}^{j}$ for any $j$ is weakly monotone in $\beta, \gamma, c^{k}$, and $w^{k}$ for any $k$ by Lemma F.3, it follows that $\left(\pi p_{N}\right)^{i}$ is weakly monotone in $\beta, \gamma, c^{i}$, and $w^{i}$. Together with Lemma H.8, this shows strict monotonicity. For monotonicty with respect to $e^{j}$, observe that (74) implies

$$
\frac{\partial p_{N}^{i}}{\partial e^{j}}=\beta \bar{\pi}^{i j} \alpha_{N} 1_{\left\{j \in \mathcal{C}_{N}\right\}}+\beta \sum_{k \in \mathcal{C}_{N}} \bar{\pi}^{i k} e^{k} \frac{\partial \alpha_{N}}{\partial e^{j}}
$$

at a differentiability point. By Lemma $\mathrm{H} .8$, this partial derivative is zero for $j \in \mathcal{S}_{N}$ and negative for $j \in \mathcal{D}_{N} \backslash \mathcal{C}_{N}$. For $j \in \mathcal{C}_{N}$, it is positive if and only if 85 holds.

Lemma H.12. For each $i$, let $e_{*}^{i}$ be as in Lemma H.7. Welfare losses $W_{N}$ are increasing in $\gamma$, non-decreasing in $\lambda$, and non-increasing in $\beta$. Welfare losses $W_{N}$ is locally increasing in $\lambda$ if and only if $\theta^{i}(\beta, \pi)>0$ for at least one bank $i \in \mathcal{D}_{N} \backslash \mathcal{C}_{N}$. Moreover, $W_{N}$ is locally decreasing in $\beta$ if and only if $\theta^{i}(\beta, \pi)>0$ for at least one bank $i \in \mathcal{D}_{N}$. Finally, suppose that $(1+\lambda) \beta \leq 1$ or 
$\mathcal{D}_{N} \backslash \mathcal{C}_{N}=\emptyset$. Then welfare losses $W_{N}$ are increasing in $e^{i}$ except at $e_{*}^{i}$, where they have a downward discontinuity.

Proof. It follows immediately from the definition of welfare losses in (6) that $W_{N}$ is non-decreasing in $\lambda$ and that it is increasing if and only if $\delta^{i}\left(p_{N}, \alpha_{N}\right)>0$ for at least one bank $i \in \mathcal{D}_{N} \backslash \mathcal{C}_{N}$. The latter condition is equivalent to $\theta^{i}(\beta, \pi)$ for one bank $i \in \mathcal{D}_{N} \backslash \mathcal{C}_{N}$. For the sensitivity with respect to the remaining parameters, note that Lemma B.1 implies that there exists a constant $C>0$ that does not depend on $\gamma, \beta$, or $e$ such that for $\tilde{\beta}:=(1+\lambda) \beta$, we obtain

$$
W_{N}=C+\sum_{i=1}^{n} e^{i}-\left(\sum_{i \in \mathcal{S}_{N}} e^{i}+\tilde{\beta} \sum_{i \in \mathcal{D}_{N} \backslash \mathcal{C}_{N}} e^{i}\right) \alpha_{N}-\sum_{j \in \mathcal{C}_{N}} p_{N}^{j}\left(\sum_{i \in \mathcal{S}_{N}} \pi^{i j}+\tilde{\beta} \sum_{i \in \mathcal{D}_{N} \backslash \mathcal{C}_{N}} \pi^{i j}\right)-\sum_{i \in \mathcal{D}_{N} \backslash \mathcal{C}_{N}} \tilde{\beta} c^{i} .
$$

It follows immediately from Lemmas H.8 and H.11 that $W_{N}$ is strictly increasing in $\gamma$ and $e^{j}$ for $j \in \mathcal{S}_{N}$ and that it is non-decreasing in $\beta$. Taking the derivative of (86) with respect to $\beta$ yields

$$
\begin{aligned}
\frac{\partial W_{N}}{\partial \beta}= & -\left(\sum_{i \in \mathcal{S}_{N}} e^{i}+\tilde{\beta} \sum_{i \in \mathcal{D}_{N} \backslash \mathcal{C}_{N}} e^{i}\right) \frac{\partial \alpha_{N}}{\partial \beta}-\sum_{j \in \mathcal{C}_{N}} \frac{\partial p_{N}^{j}}{\partial \beta}\left(\sum_{i \in \mathcal{S}_{N}} \pi^{i j}+\tilde{\beta} \sum_{i \in \mathcal{D}_{N} \backslash \mathcal{C}_{N}} \pi^{i j}\right) \\
& -\sum_{i \in \mathcal{D}_{N} \backslash \mathcal{C}_{N}}\left(c^{i}+\alpha_{N} e^{i}+\sum_{j \in \mathcal{C}_{N}} \pi^{i j} p_{N}^{j}\right) .
\end{aligned}
$$

Lemmas H.8 and H.11 imply that $\alpha_{N}$ is increasing in $\beta$ if and only if $\pi^{i j}>0$ for some $j \in \mathcal{C}_{N}$ and $i \in \mathcal{I}_{N}$, and that $p_{N}$ is increasing in $\beta$. Therefore, $W_{N}$ is strictly decreasing in $\beta$ if and only if at least one bank $j \in \mathcal{C}_{N}$ has a creditor $i \notin \mathcal{C}_{N}$ or if $\mathcal{D}_{N} \backslash \mathcal{C}_{N}$ is non-empty. The former condition is equivalent to $\theta^{i}(\beta, \pi)>0$ for at least one $i \in \mathcal{C}_{N}$ and the latter condition is equivalent to $\theta^{i}(\beta, \pi)>0$ for at least one $i \in \mathcal{D}_{N} \backslash \mathcal{C}_{N}$. This concludes the proof of the sensitivity with respect to $\beta$.

It remains to analyze the sensitivity with respect to $e^{j}$. Observe first that if $e^{j}>e_{*}^{j}$, then $j \in \mathcal{S}_{N}$ by Lemma $\mathrm{H.7}$ and $\alpha_{N}$ and hence $p_{N}$ are constant in $e^{j}$ by Lemma H.8. It follows frrom (86) that $W_{N}$ is constant in $e^{j}$. Suppose next that $e^{j}<e_{*}^{j}$ so that $j \in \mathcal{D}_{N}$ by Lemma H.7. It follows from (80) and (81) that the weak partial derivative of (86) equals

$$
\frac{\partial W_{N}}{\partial e^{j}}=1-\alpha_{N} \beta\left(\theta^{j}(\beta, \pi)+\lambda \theta_{\mathcal{D}_{N}}^{j}(\beta, \pi)\right)-\left(\sum_{i \in \mathcal{S}_{N}} e^{i}+\beta \sum_{i \in \mathcal{D}_{N}}\left(\theta^{i}(\beta, \pi)+\lambda \theta_{\mathcal{D}_{N}}^{i}(\beta, \pi)\right) e^{i}\right) \frac{\partial \alpha_{N}}{\partial e^{j}} .
$$

If $(1+\lambda) \beta \leq 1$ or if $\mathcal{D}_{N} \backslash \mathcal{C}_{N}=\emptyset$, then the sum of the first two terms in 87) is non-negative by Lemma 4.3. Since $\alpha_{N}$ is decreasing in $e^{j}$ by Lemma H.8 it follows that $W_{N}$ is increasing in $e^{j}$. At $e^{j}=e_{*}^{j}$, it follows from Lemma H.7 that bank $j$ becomes solvent and hence $W_{N}$ has a downward discontinuity. It follows in the same way as in the proof of Lemma H.8 that an increase in $e^{j}$ other than at $e_{*}^{j}$ can lead to changes in $\mathcal{D}_{N}$ only through other banks defaulting. At those points, there are upward discontinuities in $W_{N}$. 
Lemma H.13. Let $0 \leq c_{1}^{i} \leq c_{*}^{i} \leq c_{2}^{i}$ be as in Lemma H.7. Welfare losses $W_{N}$ are continuously increasing in $c^{i}$ for $c^{i} \leq c_{1}^{i}$ if and only if $(1+\lambda) \beta<1, W_{N}$ is decreasing for $c^{i} \in\left[c_{*}^{i}, c_{2}^{i}\right]$ with a downward discontinuity at $c_{*}^{i}$, and constant for $c^{i} \geq c_{2}^{i}$. On the interval $\left[c_{1}^{i}, c_{*}^{i}\right]$, welfare losses $W_{N}$ have only downward discontinuities and they are locally decreasing in $c^{i}$ if and only if

$$
\beta \theta^{i}(\beta, \pi)+\beta \lambda \theta_{\mathcal{D}_{N} \backslash \mathcal{C}_{N}}^{i}(\beta, \pi)+\gamma \theta_{\mathcal{I}}^{i}(\beta, \pi) \cdot \frac{\sum_{j \in \mathcal{S}_{N}} e^{j}+(1+\lambda) \beta \sum_{j \in \mathcal{D}_{N}} e^{j}}{1+\ln \left(\alpha_{N}\right)+\gamma \sum_{j \in \mathcal{D}_{N}} e^{j}-\gamma \beta \sum_{j \in \mathcal{C}_{N}} \theta_{\mathcal{I}}^{j} e^{j}} \geq 1 .
$$

Proof. For $\tilde{\beta}:=(1+\lambda) \beta$, we recast welfare losses in the default cascade as

$$
W_{N}=C^{\prime}+\sum_{j \in \mathcal{C}_{N}} c^{j}+\sum_{j \in \mathcal{D}_{N} \backslash \mathcal{C}_{N}}(1-\tilde{\beta}) c^{j}-\sum_{j \in \mathcal{S}_{N}}\left(e^{j} \alpha_{N}+\left(\pi p_{N}\right)^{j}\right)-\tilde{\beta} \sum_{j \in \mathcal{D}_{N} \backslash \mathcal{C}_{N}}\left(e^{j} \alpha_{N}+\left(\pi p_{N}\right)^{j}\right)
$$

for a constant $C^{\prime}>0$ which does not depend on $c$. Observe first that $\alpha_{N}$ and $p_{N}$ are constant in $c^{i}$ for $c^{i} \notin\left[c_{1}^{i}, c_{2}^{i}\right]$ by Lemma H.8 and (83). For $c^{i} \leq c_{1}^{i}$, Lemma H.7 shows that $i \in \mathcal{D}_{N} \backslash \mathcal{C}_{N}$. Thus, it follows straight from $(88)$ that $W_{N}$ is increasing in $c^{i}$ if and only if $\tilde{\beta}<1$. Similarly, for $c^{i} \geq c_{2}^{i}$, Lemma H.7 states that $i \in \mathcal{S}_{N} \backslash \mathcal{I}_{N}$, hence $W_{N}$ is locally constant by 88). Suppose now that $c^{i} \in\left[c_{1}^{i}, c_{2}^{i}\right]$. Because prices are non-decreasing in $c^{i}$ by Lemma F.3, an increase in $c^{i}$ can affect solvency of other banks only by making them solvent, leading to downward discontinuities in $W_{N}$. At continuity points, welfare losses are weakly differentiable. It follows from (74) that

$$
\frac{\partial\left(\pi p_{N}\right)^{j}}{\partial c^{i}}=\beta \pi^{j, \mathcal{C}_{N}}\left(I-\beta \pi^{\mathcal{C}_{N}, \mathcal{C}_{N}}\right)^{-1}\left(\rho_{i}^{\mathcal{C}_{N}} 1_{\left\{c^{i}<c_{*}^{i}\right\}}+e^{\mathcal{C}_{N}} \frac{\partial \alpha_{N}}{\partial c^{i}}\right)
$$

where we have used that $i \in \mathcal{C}_{N}$ if and only if $c^{i} \in\left[c_{1}^{i}, c_{*}^{i}\right)$ by Lemma H.7. Taking the weak partial derivative of 88 with respect to $c^{i}$ thus yields

$$
\frac{\partial W_{N}}{\partial c^{i}}=\left(1-\beta \theta^{i}(\beta, \pi)-\beta \lambda \theta_{\mathcal{D}_{N} \backslash \mathcal{C}_{N}}^{i}(\beta, \pi)\right) 1_{\left\{c^{i}<c_{*}^{i}\right\}}-\left(\sum_{j \in \mathcal{S}_{N}} e^{j}+\tilde{\beta} \sum_{j \in \mathcal{D}_{N}} \theta^{j}(\beta, \pi) e^{j}\right) \frac{\partial \alpha_{N}}{\partial c^{i}} .
$$

Consider first the case where $c^{i} \in\left[c_{*}^{i}, c_{2}^{i}\right]$. Since $\alpha_{N}$ is increasing in $c^{i}$ by Lemma H.8, it follows from (89) that $W_{N}$ is decreasing in $c^{i}$. For $c^{i} \in\left[c_{1}^{i}, c_{*}^{i}\right)$, the statement follows from (84), 89), and Lemma $\underline{\text { H.10. }}$

\section{H.3 Credibility of the Threat}

Lemma H.14. Let $0 \leq \gamma_{1} \leq \gamma_{2}$ be as in Lemma H.2. For $\gamma \geq \gamma_{2}$, the difference $W_{P}-W_{N}$ is decreasing in $\gamma$. For $\gamma \leq \gamma_{2}$, an increase in $\gamma$ leads to downward discontinuities in $W_{P}-W_{N}$ when additional banks default. At continuity points, $W_{P}-W_{N}$ is locally increasing in $\gamma$ if and only if

$$
\frac{\sum_{i \in \mathcal{S}_{N}} e^{i}+(1+\lambda) \beta \sum_{i \in \mathcal{D}_{N}} \theta^{i}(\beta, \pi) e^{i}}{\frac{1+\ln \left(\alpha_{N}\right)}{\gamma}+\sum_{i \in \mathcal{D}_{N}} e^{i}-\beta \sum_{i \in \mathcal{C}_{N}} \theta_{\mathcal{I}_{N}}^{i} e^{i}} \geq \lambda \frac{\alpha_{P} \ln \left(\alpha_{P}\right)}{\alpha_{N} \ln \left(\alpha_{N}\right)}+\frac{\lambda\left(1+\ln \left(\alpha_{P}\right)\right)-\gamma \sum_{i=1}^{n} e^{i}}{1+\ln \left(\alpha_{P}\right)-\gamma \sum_{i \in \mathcal{F}} e^{i}} 1_{\left\{\gamma \leq \gamma_{1}\right\}} .
$$


Proof. Observe first that $W_{P}$ is constant for $\gamma \geq \gamma_{2}$, hence $W_{P}-W_{N}$ is decreasing by Lemma H.12. Moreover, continuity of $W_{P}$ implies via Lemma $\mathrm{H}$.12 that all discontinuities of $W_{P}-W_{N}$ are downward discontinuities. Suppose now that $\gamma \leq \gamma_{2}$. Taking the partial derivative in 86 with respect to $\gamma$ yields

$$
\frac{\partial W_{N}}{\partial \gamma}=-\frac{\alpha_{N} \ln \left(\alpha_{N}\right)}{\gamma\left(1-\chi_{N}\right)}\left(\sum_{i \in \mathcal{S}_{N}} e^{i}+(1+\lambda) \beta \sum_{i \in \mathcal{D}_{N}} \theta^{i}(\beta, \pi) e^{i}\right) .
$$

The statement thus follows from (71) with some careful arithmetics.

Proof of Lemma 4.4. Observe first that welfare losses $W_{P}$ in the public bailout do not depend on $\beta$. The desired statement thus follows straight from Lemma H.12. For the sensitivity with respect to $\lambda$, observe that the marginal decrease of $\sum_{j \in \mathcal{D}_{N}(s)} \delta^{j}(s)$ with respect to the provided subsidy $s^{i}$ is $\theta_{\mathcal{D}_{N} \backslash \mathcal{C}_{N}}^{i}(\beta, \pi) \leq 1$. For $s=s_{L}$, no bank defaults and hence $\delta\left(s_{L}\right)=0$. It follows that

$$
\frac{\partial W_{N}}{\partial \lambda}=\sum_{j \in \mathcal{D}_{N} \backslash \mathcal{C}_{N}} \delta^{j}\left(p_{N}, \alpha_{N}\right)<\sum_{i=1}^{n} s_{L}^{i} \leq \sum_{i=1}^{n} s_{0}^{i}+\frac{\alpha_{P} \ln \left(\alpha_{P}\right)}{\gamma}=\frac{\partial W_{P}}{\partial \lambda},
$$

where the first inequality is strict since $e^{i}>0$ for every bank $i$ by assumption and we have used (70) in the last equality.

For the sensitivity with respect to $e^{i}$, observe that $W_{P}$ is continuous with respect to $e^{i}$ and, by Lemma H.12, $W_{N}$ has downward discontinuities except at $e_{*}^{i}$. Therefore, the only upward discontinuity of $W_{P}-W_{N}$ occurs at $e_{*}^{i}$. At continuity points, $W_{P}-W_{N}$ is weakly differentiable. It follows from (87) that $\frac{\partial W_{N}}{\partial e^{i}} \geq 1-\alpha_{N}$ if $(1+\lambda) \beta \leq 1$ or if $\mathcal{D}_{N} \backslash \mathcal{C}_{N}=\emptyset$. Together with $(73)$, this shows that

$$
\frac{\partial\left(W_{P}-W_{N}\right)}{\partial e^{i}} \leq \alpha_{N}-\alpha_{P}<0,
$$

where we have used that $\alpha_{P} \geq \alpha_{L}>\alpha_{N}$ by Lemma F.3. Note that $\alpha_{L}$ is strictly larger than $\alpha_{N}$ because there is at least one fundamentally defaulting bank.

Finally, it follows from Lemma H.14 that there exists $\gamma_{2}$ such that $W_{P}-W_{N}$ is decreasing for $\gamma \geq \gamma_{2}$ and that all discontinuity points are downward discontinuities. Since $W_{P}$ does not depend on $\beta$ or $\theta^{i}(\beta, \pi)$, it follows from (90) that $\frac{\partial\left(W_{P}-W_{N}\right)}{\partial \gamma}$ is decreasing in $\theta^{i}(\beta, \pi)$ for any bank $i \in \mathcal{C}_{N}$. Since $\theta^{i}(\beta, \pi)$ is increasing in $\beta$, it also follows that $\frac{\partial\left(W_{P}-W_{N}\right)}{\partial \gamma}$ is decreasing in $\beta$.

Proof of Lemma 4.5. We first show monotonicity of $W_{P}-W_{N}$ in $c^{i}$. Monotonicity in $\varepsilon^{i}$ then follows from $c^{i}=c_{0}^{i}-\varepsilon^{i}$ via the chain rule. Recall first that $W_{P}$ is continuous and $W_{N}$ has only downward discontinuities by Lemma H.13. Therefore, $W_{P}-W_{N}$ has only upward discontinuities. We deduce in the same way as in the proof of Lemma H.7 that there exists $0 \leq c_{a}^{i} \leq c_{b}^{i}$ such that $i$ does not have to liquidate anything in the clearing equilibrium $\left(L, \ell_{L}, \alpha_{L}\right)$ if and only if $c^{i} \geq c_{b}^{i}$ and that $i \in \mathcal{F}$ if and only if $c^{i}<c_{a}^{i}$. Since interbank claims are valued higher in $\left(L, \ell_{L}, \alpha_{L}\right)$ than in $\left(p_{N}, \ell_{N}, \alpha_{N}\right)$, more cash is required in the latter clearing equilibrium to reach solvency and liquidity, that is, $c_{2}^{i} \geq c_{b}^{i}$ and $c_{*}^{i} \geq c_{a}^{i}$, where $c_{2}^{i}$ and $c_{*}^{i}$ are defined in Lemma H.7. By definition of $c_{b}^{i}$ we can write 
$s_{0}^{i}=\left(c_{b}^{i}-c^{i}\right)^{+}$and hence $-\frac{\partial s_{0}^{i}}{\partial w^{i}}=1_{\left\{c^{i} \leq c_{b}^{i}\right\}}$. Since the complete bailout depends on $c^{i}$ only through $s_{0}^{i}$ it follows that $W_{P}$ and $\alpha_{P}$ are constant in $c^{i}$ for $c^{i} \geq c_{b}^{i}$ and that $\frac{\partial \alpha_{P}}{\partial c^{i}}=-\frac{\partial \alpha_{P}}{\partial s_{0}^{i}}$ and $\frac{\partial W_{P}}{\partial c^{i}}=-\frac{\partial W_{P}}{\partial s_{0}^{i}}$ otherwise. It follows from $(72)$ and Lemma H.1 that

$$
\frac{\partial W_{P}}{\partial c^{i}}=-\lambda\left(1-\frac{\left(\ln \left(\alpha_{L}\right)-\ln \left(\alpha_{\text {ind }}\right)\right)^{+}}{1+\ln \left(\alpha_{L}\right)+\gamma \sum_{j \in \mathcal{F}} e^{j}} 1_{\left\{c^{i}>c_{a}^{i}\right\}}\right) 1_{\left\{c^{i} \leq c_{b}^{i}\right\}} .
$$

It follows in the same was as in the proof of Lemma H.5 that this quantity is between $-\lambda$ and 0 .

Since $W_{N}$ is decreasing for $c^{i} \in\left[c_{1}^{i}, c_{2}^{i}\right]$ and constant for $c^{i} \geq c_{2}^{i}$ by Lemma H.13, it follows immediately from (91) that $W_{P}-W_{N}$ id increasing for $c^{i} \in\left[\max \left(c_{1}^{i}, c_{b}^{i}\right), c_{2}^{i}\right]$ and constant for $c^{i} \geq c_{2}^{i}$. Since $\frac{\partial W_{N}}{\partial c^{i}}=1-(1+\lambda) \beta$ for $c^{i} \leq c_{1}^{i}$, it follows from (91) that $\frac{\partial\left(W_{P}-W_{N}\right)}{\partial c^{i}}=-(1+\lambda)(1-\beta)<0$ for $c^{i} \in\left[0, \min \left(c_{1}^{i}, c_{a}^{i}\right)\right]$. For $c^{i} \in\left[c_{1}^{i}, c_{b}^{i}\right]$, the partial derivative of $W_{N}$ with respect to $c^{i}$ is given by (89). In particular, $\frac{\partial W_{N}}{\partial c^{i}}$ is decreasing in $\beta$ and $\theta^{j}(\beta, \pi)$ for any $j \in \mathcal{C}_{N}$. For $c^{i} \in\left[c_{a}^{i}, c_{1}^{i}\right]$, the partial derivative of $W_{N}$ is given by $1-(1+\lambda) \beta$, which is also decreasing in $\beta$ and trivially non-increasing in $\theta^{i}(\beta, \pi)$. Since $\frac{\partial W_{P}}{\partial c^{i}}$ is constant in $\beta$ and $\theta^{j}(\beta, \pi)$, it follows that $\frac{\partial\left(W_{P}-W_{N}\right)}{\partial c^{i}}$ is increasing in $\beta$ and $\theta^{j}(\beta, \pi)$ for any $j \in \mathcal{C}_{N}$ and decreasing in $\lambda$. The result thus follows from the chain rule and by setting $\varepsilon_{1}^{i}:=c_{0}^{i}-c_{2}^{i}, \varepsilon_{2}^{i}:=c_{0}^{i}-\max \left(c_{b}^{i}, c_{1}^{i}\right)$, and $\varepsilon_{3}^{i}:=c_{0}^{i}-\max \left(c_{1}^{i}, c_{a}^{i}\right)$.

We conclude this section by showing that the throughput is a conditionally sufficient statistic for the network structure.

Proof of Lemma 4.3. It follows as an application of Lemma $H .9$ to $y^{\mathcal{C}}=\rho_{i}^{\mathcal{C}_{N}}$ that $\theta^{i}(\beta, \pi) \in[0,1]$ for any bank $i$. Monotonicity in $\beta$ follows immediately from $\left(I-\beta \pi^{\mathcal{C}_{N}, \mathcal{C}_{N}}\right)^{-1}=\sum_{k=0}^{\infty}\left(\beta \pi^{\mathcal{C}_{N}, \mathcal{C}_{N}}\right)^{k}$. For the second statement, consider $\mathcal{D}=\mathcal{D}_{N}, \mathcal{C}=\mathcal{C}_{N}, \mathcal{S}=\mathcal{D}_{N}^{c}$, and $\mathcal{I}=\mathcal{I}_{N}$ as fixed. Observe from (78) that the asset price $\alpha_{N}$ depends on $p_{N}$ only through $\pi p_{N}$. It follows from (74) that

$$
\left(\pi p_{N}\right)^{i}=\sum_{j \in \mathcal{C}} \theta_{i}^{j}(\beta, \pi)\left(\beta c^{j}+\beta \alpha_{N} e^{j}+\beta \sum_{k \in \mathcal{S}} L^{j k}-w^{j}\right)+\sum_{j \in \mathcal{S}} L^{i j}
$$

for any bank $i \notin \mathcal{C}_{N}$, showing that $\left(\pi p_{N}\right)^{i}$ depends on $\pi$ only through $\sum_{j \in \mathcal{C}} \theta_{i}^{j}(\beta, \pi)$ and $\sum_{j \in \mathcal{S}} L^{i j}$. Therefore, (78) implies that $\alpha_{N}$ depends on $\pi$ only through $\sum_{j \in \mathcal{C}} \theta_{\mathcal{I}}^{j}(\beta, \pi)$ and $\sum_{j \in \mathcal{S}} L^{i j}$. Finally, it follows as a consequence to 28 that $W_{N}$ depends on $\pi$ only through $\sum_{j \in \mathcal{C}} \theta_{\mathcal{I}}^{j}(\beta, \pi)$, the total throughput $\sum_{j \in \mathcal{C}} \theta^{j}(\beta, \pi)$, and $\sum_{j \in \mathcal{S}} L^{i j}$. Since the complete bailout does not depend on the network structure at all, the claim follows.

\section{Proofs of Section 5}

Proof of Lemma 5.1. Let $s$ be any set of subsidies that rescues banks in $\mathcal{B}$ by awarding direct subsidies only to banks in $\mathcal{B}$. For any such vector of subsidies, the greatest clearing payment vector is given by $\bar{p}(s)=p(\mathcal{B})$. Indeed, for any such vector of subsidies, banks in $\mathcal{B}$ are able to repay their liabilities in full. Contingent on full repayment by banks in $\mathcal{B}$, banks in $\mathcal{B}^{c}$ are not affected by 
the subsidies as they are awarded only to banks in $\mathcal{B}$, hence $\bar{p}(s)=p(\mathcal{B})$. The welfare-maximizing vector of direct subsidies that banks $i \in \mathcal{B}$ require to afford payment $L^{i}$ is thus given by the shortfall $\left(L^{i}+w^{i}-c^{i}-(\pi p(\mathcal{B}))^{i}\right)^{+}$. This shows that any welfare-maximizing bailout is of the form $s(\mathcal{B})$ for a suitable set $\mathcal{B}$, hence it is in $\mathcal{S}_{P}$.

Proof of Lemma 5.3. Fix a feasible proposal $(b, s)$ with accepting equilibrium response $a$. Necessity of Condition 1 follows in the same way as in the proof of Lemma 3.5. Therefore, the regulator's best response after rejection by any bank $i$ in $\mathcal{C}:=\left\{i \mid b^{i}>0, a^{i}=1\right\}$ is a public bailout. Let $\mathcal{B}_{i} \in \mathcal{B}_{P}$ be such that $r\left(b, s,\left(0, a^{-i}\right)\right)=s\left(\mathcal{B}_{i}\right)$. Suppose now that $a^{i}=1$ but Condition 2 is violated for bank $i$ with threats from $s\left(\mathcal{B}_{i}\right)$. The negation of Condition 2 implies that

$$
L^{i}+w^{i} \leq c^{i}+s^{i}-b^{i}+\sum_{j=1}^{n} \pi^{i j} L^{j}<c^{i}+s^{i}\left(\mathcal{B}_{*}\right)+\sum_{j=1}^{n} \pi^{i j} p^{j}\left(\mathcal{B}_{i}\right) .
$$

This shows that bank $i$ is solvent in the optimal bailout $s\left(\mathcal{B}_{i}\right)$. Therefore, subtracting $L^{i}+w^{i}$ in 92 ) implies that $V^{i}\left(s\left(\mathcal{B}_{i}\right)\right)>V^{i}\left(b, s,\left(1, a^{-i}\right)\right)$. This is a contradiction. Sufficiency of the conditions follows analogously to the proof of Lemma 3.5 .

For the second statement, suppose that there exists $\mathcal{B}_{*} \in \mathcal{B}_{P}$ such that Condition 2 holds in the accepting equilibrium $a$ for every bank $i \in \mathcal{C}$ when the bailout $s\left(\mathcal{B}_{*}\right)$ is threatened. Define the regulator's reaction $\tilde{r}$ by setting $\tilde{r}(b, s, \tilde{a})=$ "bailout $\mathcal{B}_{*}$ " for any $\tilde{a}$ with $W_{\lambda}(b, s, \tilde{a})>W_{P}^{*}$ and $\tilde{r}(b, s, \tilde{a})=$ "bail-in" otherwise. Reaction $\tilde{r}$ is an equilibrium reaction that leads to the public bailout $s\left(\mathcal{B}_{*}\right)$ in all rejecting equilibria. Condition 2 implies that $V^{i}(b, s, a) \geq V^{i}\left(s\left(\mathcal{B}_{*}\right)\right)$ for every bank $i \in \mathcal{C}$. Since $a$ is an accepting equilibrium, by definition $W_{\lambda}(b, s, a) \leq W_{P}^{*}$, hence a rejecting equilibrium is subgame Pareto efficient only if $(b, s)$ is equivalent to some public bailout of Lemma 5.1.

For the converse, fix a rejecting equilibrium $\tilde{a}$. Let $\mathcal{B}_{*} \in \mathcal{B}_{P}$ be an arbitrary bailout that is implemented in reaction to $\tilde{a}$. Let $a$ be any accepting equilibrium with contributing banks $\mathcal{C}$. By assumption, there exists a bank $i \in \mathcal{C}$ for which Condition 2 is violated for $\mathcal{B}_{i}=\mathcal{B}_{*}$. Therefore, bank $i$ is strictly worse off in $a$ than in $\tilde{a}$, showing that $\tilde{a}$ cannot be Pareto dominated by an accepting equilibrium.

\section{I.1 Proof of Theorem 5.4}

In addition to proving Theorem 5.4. we also characterize the bail-in proposals that attain the equilibrium welfare losses stated in Theorem 5.4 .

Proposition I.1. For any $\mathcal{B}_{*} \in \mathcal{B}_{P}$, let $\Xi_{\mathcal{B}_{*}}(\mathcal{B})$ denote the set of bail-in proposals $(b, s)$ such that:

1. if $W_{\mathcal{B}_{*}}(\mathcal{B})=W_{\lambda}(s(\mathcal{B}))-\sum_{j=1}^{m_{\mathcal{B}_{*}}(\mathcal{B})} \nu^{i_{\mathcal{B}_{*}}^{j}(\mathcal{B})}(\mathcal{B})$, then

(a) $s=s(\mathcal{B})$

(b) $b^{j}=\eta_{\mathcal{B}_{*}}^{j}(\mathcal{B})$ for $j=i_{\mathcal{B}_{*}}^{1}(\mathcal{B}), \ldots, i_{\mathcal{B}_{*}}^{m_{\mathcal{B}_{*}}(\mathcal{B})}(\mathcal{B})$ and $b^{j}=0$ otherwise.

2. If $W_{\mathcal{B}_{*}}(\mathcal{B})=W_{P}^{*}-\lambda \eta^{i_{m_{\mathcal{B}_{*}}(\mathcal{B})+1}(\mathcal{B})}(\mathcal{B})$, then 
(a) $s \geq s(\mathcal{B})$,

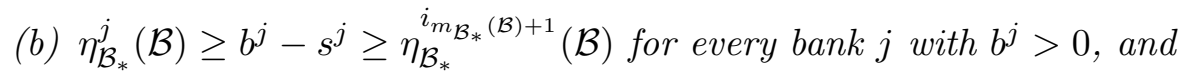

(c) $\sum_{i=1}^{n}\left(s^{i}-s^{i}(\mathcal{B})\right)=\sum_{j=1}^{m_{\mathcal{B}_{*}}(\mathcal{B})} \eta_{\mathcal{B}_{*}}^{i_{\mathcal{B}_{*}}^{j}(\mathcal{B})}(\mathcal{B})+\frac{1}{\lambda}\left(W_{P}^{*}-W_{\lambda}(s(\mathcal{B}))\right)$

In any subgame Pareto efficient equilibrium with welfare losses $W_{\mathcal{B}_{*}}(\mathcal{B})$, a bail-in from $\Xi_{\mathcal{B}_{*}}(\mathcal{B})$ is proposed and accepted by all banks.

We proceed to prove Theorem 5.4 and Proposition I.1 in parallel. We start by giving two precursory lemmas, which will be invoked in their proof.

Lemma I.2. Any $(b, s) \in \Xi_{\mathcal{B}_{*}}(\mathcal{B})$ admits a unique accepting continuation equilibrium a with $a^{i}=1$ for every bank $i$ and $W_{\lambda}(b, s, a)=W_{\mathcal{B}_{*}}(\mathcal{B})$.

Proof. Fix $\mathcal{B}_{*} \in \mathcal{B}_{P}$ and a set of banks $\mathcal{B}$ to be rescued. For the sake of brevity, denote $\eta=\eta_{\mathcal{B}_{*}}(\mathcal{B})$, $i_{j}=i_{\mathcal{B}_{*}}^{j}(\mathcal{B})$, and $m=m_{\mathcal{B}_{*}}(\mathcal{B})$. Consider a bail-in proposal $(b, s) \in \Xi_{\mathcal{B}_{*}}(\mathcal{B})$ with response vector $a=(1, \ldots, 1)$. Suppose first that $W_{\mathcal{B}_{*}}(\mathcal{B})=W_{\lambda}(s(\mathcal{B}))-\lambda \sum_{j=1}^{m} \eta^{i_{j}}(\mathcal{B})$. Then the definition of $\Xi_{\mathcal{B}_{*}}(\mathcal{B})$ in Proposition I.1 imposes that $s=s(\mathcal{B})$ and $b^{j}=\eta^{j}$ for any bank $j \in\left\{i_{1}, \ldots, i_{m}\right\}$. It follows straight from (7) that $W_{\lambda}(b, s, a)=W_{\mathcal{B}_{*}}(\mathcal{B})$. Suppose, therefore, that $W_{\mathcal{B}_{*}}(\mathcal{B})=W_{P}^{*}-\lambda \eta^{i_{m+1}}(\mathcal{B})$ instead. Then we obtain

$$
W_{\lambda}(b, s, a)=W_{\lambda}(s(\mathcal{B}))+\lambda \sum_{i=1}^{n}\left(s^{i}-s^{i}(\mathcal{B})\right)-\lambda \sum_{j=1}^{m+1} \eta^{i_{j}}=W_{P}^{*}-\lambda \eta^{i_{m+1}}
$$

where we have used Property 2.(c) of a bail-in in $\Xi_{\mathcal{B}_{*}}(\mathcal{B})$ in the last equation.

To see that $a$ is indeed a continuation equilibrium, we verify the necessary and sufficient conditions given in Lemma 5.3. By definition of $\Xi_{\mathcal{B}_{*}}(\mathcal{B})$, any bank $i$ 's net contribution $b^{i}-s^{i}$ is smaller than its maximal incentive-compatible contribution $\eta_{\mathcal{B}_{*}}^{i}(\mathcal{B})$ given in 23 . Therefore, the second condition in Lemma 5.3 is satisfied for every bank. For the first condition, we distinguish again the two cases. If $W_{\mathcal{B}_{*}}(\mathcal{B})=W_{\lambda}(s(\mathcal{B}))-\lambda \sum_{j=1}^{m} \eta^{i_{j}}$, then $b^{j}=\eta^{j}$ for $j \in\left\{i_{1}, \ldots, i_{m}\right\}$. Thus, a deviation by bank $i_{k}$ for $k=1, \ldots, m$ would lead to welfare losses of

$$
W_{\lambda}\left(b, s,\left(0, a^{-i_{k}}\right)\right)=W_{\lambda}(b, s, a)+\lambda \eta^{i_{k}} \geq W_{\lambda}(b, s, a)+\lambda \eta^{i_{m}} \geq W_{P}^{*}
$$

where the first inequality holds because $i_{1}, \ldots, i_{m}$ is a decreasing order of the components of $\eta$ and the second inequality holds by definition of $m$ given in Theorem 5.4. This shows that Condition 1 in Lemma 5.3 is satisfied and hence $a$ is indeed an equilibrium response. It also implies that the regulator will not agree to proceed with the bail-in if only a subset of banks accepts, thereby showing uniqueness. If $W_{\mathcal{B}_{*}}(\mathcal{B})=W_{P}^{*}-\lambda \eta^{i_{m+1}}(\mathcal{B})$ holds instead, then $b^{i} \geq b^{i}-s^{i} \geq \eta^{i_{m+1}}$ by Property 2.(b), hence

$$
W_{\lambda}\left(b, s,\left(0, a^{-i_{k}}\right)\right)=W_{\lambda}(b, s, a)+\lambda \eta^{i_{k}} \geq W_{\lambda}(b, s, a)+\lambda \eta^{i_{m+1}}=W_{P}^{*}
$$

The remainder of the argument works analogously. 
Lemma I.3. Let $(b, s)$ be a feasible bail-in with equilibrium response a when threats come from the bailout $s\left(\mathcal{B}_{*}\right)$ for $\mathcal{B}_{*} \in \mathcal{B}_{P}$. Let $\mathcal{B}$ denote the set of solvent banks in $(b, s, a)$ with $s^{i}>0$. Then $W_{\lambda}(b, s, a) \geq W_{\mathcal{B}_{*}}(\mathcal{B})$, where the inequality is binding only if $(b, s) \in \Xi_{\mathcal{B}_{*}}(\mathcal{B})$.

Proof. Fix $\mathcal{B}_{*} \in \mathcal{B}_{P}$. Let $(b, s)$ be a bail-in with continuation equilibrium $a$ if threats come from the bailout $s\left(\mathcal{B}_{*}\right)$. Let $\mathcal{B}:=\left\{i \mid s^{i}>0, \bar{p}^{i}(b, s, a)=L^{i}\right\}$ and abbreviate $\eta=\eta_{\mathcal{B}_{*}}(\mathcal{B})$ and $m=m_{\mathcal{B}_{*}}(\mathcal{B})$. If $a$ is a rejecting equilibrium, then $W_{\lambda}(b, s, a)=W_{P}^{*}$. Thus, it follows straight from the definitions of $m$ and $W_{\mathcal{B}_{*}}(\mathcal{B})$ in Theorem 5.4 that welfare losses are bounded from below by $W_{\mathcal{B}_{*}}(\mathcal{B})$. Suppose, therefore, that $a$ is an accepting continuation equilibrium. Due to Lemma C.1, we may assume without loss of generality that $b^{i} s^{i}=0$ and $a^{i}=1$ for any bank $i$ by passing to an equivalent equilibrium. Condition 1 of Lemma 5.3 implies that

$$
W_{\lambda}(b, s, a) \geq W_{P}^{*}-\lambda b^{i}
$$

for any contributing bank $i$. Condition 2 of Lemma 5.3 together with feasibility implies that $b^{i} \leq \eta^{i}$. Moreover, since $s(\mathcal{B})$ are the minimal subsidies that guarantee solvency of banks in $\mathcal{B}$, it must hold that

$$
\sum_{i=1}^{n} s^{i} \geq \sum_{i=1}^{n} s^{i}(\mathcal{B})
$$

We distinguish cases according to the number $k$ of contributing banks. Suppose first that $k<m$. It follows from (94) that

$$
W_{\lambda}(b, s, a) \geq W_{\lambda}(s(\mathcal{B}))-\lambda \sum_{k=1}^{m-1} \eta^{i_{k}}(\mathcal{B})>W_{P}^{*},
$$

where we have used in the last inequality that the sum of incentive-compatible contributions by $k \leq m-1$ banks must be smaller than the contributions of $i_{1}, \ldots, i_{m-1}$ in $\eta$. This contradicts the fact that $a$ is an accepting equilibrium. Suppose next that $k=m$. Again, the sum of incentivecompatible contributions by $m$ banks must be smaller than the contributions of $i_{1}, \ldots, i_{m-1}$ in $\eta$. Together with (94), this implies

$$
W_{\lambda}(b, s, a) \geq W_{\lambda}(s(\mathcal{B}))-\lambda \sum_{j=1}^{m} \eta^{i_{j}} \geq W_{\mathcal{B}_{*}}(\mathcal{B}),
$$

where the last inequality follows directly from (24). Finally, suppose that the set of contributing banks $\mathcal{C}$ is of size $k \geq m+1$. Because $i_{1}, i_{2}, \ldots$ is a non-increasing ordering of the incentivecompatible contributions, it follows that there must be a contributing bank with $b^{i} \leq \eta^{i_{m+1}}$. It thus follows from (93) that

$$
W_{\lambda}(b, s, a) \geq W_{P}^{*}-\min _{i \in \mathcal{C}} \lambda b^{i} \geq W_{P}^{*}-\lambda \eta^{i_{m+1}} \geq W_{\mathcal{B}_{*}}(\mathcal{B})
$$

This concludes the proof that no bail-in can attain equilibrium welfare losses below $W_{\mathcal{B}_{*}}(\mathcal{B})$. 
Lemma I.2 shows that welfare losses can be attained by bail-in proposals in $\Xi_{\mathcal{B}_{*}}(\mathcal{B})$. Finally, it is clear from $(95)$ and $(96)$ that these inequalities hold with equality if and only if $(b, s) \in \Xi_{\mathcal{B}_{*}}(\mathcal{B})$.

Proof of Theorem 5.4 and Proposition I.1. Lemma 5.3 shows that in order to rule out rejecting equilibria, the regulator must threaten the same optimal bailout to all banks. Fix now any $\mathcal{B}_{*} \in$ $\arg \min W_{\lambda}(s(\mathcal{B}))$ and any $\mathcal{B} \subseteq\{1, \ldots, n\}$. Lemma $I .2$ shows that any $(b, s) \in \Xi_{\mathcal{B}_{*}}(\mathcal{B})$ has a unique accepting equilibrium with welfare losses equal to $W_{\mathcal{B}_{*}}(\mathcal{B})$. By Lemma 5.3 , that accepting equilibrium is the unique subgame Pareto efficient continuation equilibrium. The regulator is thus aware that any bail-in from $\Xi_{\mathcal{B}_{*}}(\mathcal{B})$ that he proposes in the first stage will be implemented in equilibrium. Moreover, by Lemma I.3, an accepted bail-in proposal from $\Xi_{\mathcal{B}_{*}}(\mathcal{B})$ is strictly preferred by the regulator over any other accepted proposal $\left(b^{\prime}, s^{\prime}\right)$ that rescues banks in $\mathcal{B}$ by awarding direct subsidies only to banks in $\mathcal{B}$ when $s\left(\mathcal{B}_{*}\right)$ is threatened. Therefore, if $\min _{\mathcal{B}_{*}, \mathcal{B}} W_{\mathcal{B}_{*}}(\mathcal{B}) \leq W_{P}^{*}$, the regulator's only rational choice in stage 1 is to propose a bail-in from Proposition I.1. If, on the other hand, $\min _{\mathcal{B}_{*}, \mathcal{B}} W_{\mathcal{B}_{*}}(\mathcal{B})>W_{P}^{*}$, then the regulator will implement an optimal public bailout from Lemma 5.1 either by proposing it in the first stage or by choosing it in the third stage after an arbitrary proposal in the first stage.

\section{I.2 Properties of Optimal Bailout/Bail-in}

Proof of Lemma 5.2. Fix two sets of banks $\mathcal{B}^{\prime} \subseteq \mathcal{B}$. We will compare welfare losses between the bailouts $s(\mathcal{B})$ and $s\left(\mathcal{B}^{\prime}\right)$. Since $\gamma=0$, the welfare losses consist only of bankruptcy costs as well as welfare costs of taxpayer contributions and senior creditor's losses. We start by comparing the subsidies. For $\mathcal{C} \in\left\{\mathcal{B}, \mathcal{B}^{\prime}\right\}$, let $y(\mathcal{C}):=L+w-c-\pi p(\mathcal{C})$ so that $S(\mathcal{C})=y(\mathcal{C})^{+}$and $C(\mathcal{C})=y(\mathcal{C})^{-}$, where $S$ and $C$ are defined in $(21)$. Since $y(\mathcal{B})=y\left(\mathcal{B}^{\prime}\right)-\zeta$, it follows that for any $i \in \mathcal{S}(\mathcal{B})$, we have

$$
s^{i}(\mathcal{B})=S^{i}(\mathcal{B})=y^{i}(\mathcal{B})+C^{i}(\mathcal{B})=S^{i}\left(\mathcal{B}^{\prime}\right)-\zeta+C^{i}(\mathcal{B})-C^{i}\left(\mathcal{B}^{\prime}\right)
$$

Since $\mathcal{B}^{\prime} \subseteq \mathcal{B}$ and $p(\cdot)$ is monotonic in the set of banks $\mathcal{B}$, it follows that $\zeta^{i} \geq 0$ for every bank $i$. Therefore, $C^{i}\left(\mathcal{B}^{\prime}\right)=\left(-y^{i}(\mathcal{B})-\zeta^{i}\right)^{+}=\left(C^{i}(\mathcal{B})-\zeta^{i}\right)^{+}$, where we have used that $-y^{i}(\mathcal{B})-\zeta^{i}>0$ only if $y^{i}(\mathcal{B})<0$. It follows from the elementary identity $a-(a-b)^{+}=\min (a, b)$ that $C^{i}(\mathcal{B})-C^{i}\left(\mathcal{B}^{\prime}\right)=$ $\min \left(\zeta^{i}, C^{i}(\mathcal{B})\right)$. Together with 97$)$, this implies that

$$
\sum_{i \in \mathcal{S}(\mathcal{B})}\left(s^{i}\left(\mathcal{B}^{\prime}\right)-s^{i}(\mathcal{B})\right)=\sum_{i \in \mathcal{S}(\mathcal{B})}\left(\zeta^{i}-\min \left(\zeta^{i}, C^{i}(\mathcal{B})\right)\right)-\sum_{i \in \mathcal{R}} S^{i}\left(\mathcal{B}^{\prime}\right)
$$

where we have used that $s^{i}\left(\mathcal{B}^{\prime}\right)=S^{i}\left(\mathcal{B}^{\prime}\right)$ for $i \in \mathcal{S}\left(\mathcal{B}^{\prime}\right)$ and $s^{i}\left(\mathcal{B}^{\prime}\right)=0$ for $i \in \mathcal{B} \backslash \mathcal{B}^{\prime}$. For the

losses of senior creditors, note that $\delta^{i}(\mathcal{B})=\left(\delta^{i}\left(\mathcal{B}^{\prime}\right)-\beta \zeta^{i}\right)^{+}$follows straight from (5). Therefore, the elementary identity $a-(a-b)^{+}=\min (a, b)$ implies

$$
\delta^{i}\left(\mathcal{B}^{\prime}\right)-\delta^{i}(\mathcal{B})=\min \left(\beta \zeta^{i}, \delta^{i}\left(\mathcal{B}^{\prime}\right)\right)
$$


For any set of banks $\mathcal{C}$, let $g^{i}(\mathcal{C}):=(1-\beta)\left(c^{i}+\alpha e^{i}+(\pi p(\mathcal{C}))^{i}\right)$ denote the bankruptcy losses of a bank $i \in \mathcal{D}(\mathcal{C})$. Observe that 30 implies

$$
g^{i}(\mathcal{C})=c^{i}-w^{i}+(\pi p(\mathcal{C}))^{i}-p(\mathcal{C})^{i}+\delta^{i}(\mathcal{C})=L^{i}-p^{i}(\mathcal{C})+\delta^{i}(\mathcal{C})-y^{i}(\mathcal{C})
$$

Applying 100 for $\mathcal{C}=\mathcal{B}$ as well as $\mathcal{C}=\mathcal{B}^{\prime}$, we obtain

$$
g^{i}\left(\mathcal{B}^{\prime}\right)-g^{i}(\mathcal{B})=p^{i}(\mathcal{B})-p^{i}\left(\mathcal{B}^{\prime}\right)+\delta^{i}\left(\mathcal{B}^{\prime}\right)-\delta^{i}(\mathcal{B})-\zeta^{i}
$$

for any bank $i \in \mathcal{D}(\mathcal{B})$. Since any bank $i \in \mathcal{R}$ is rescued in the bailout $s(\mathcal{B})$, we have $g^{i}(\mathcal{B})=0$ and $p^{i}(\mathcal{B})=L^{i}$. Equation 100 thus implies that $g^{i}\left(\mathcal{B}^{\prime}\right)-g^{i}(\mathcal{B})=p^{i}(\mathcal{B})-p^{i}\left(\mathcal{B}^{\prime}\right)+\delta^{i}\left(\mathcal{B}^{\prime}\right)-S^{i}\left(\mathcal{B}^{\prime}\right)$ for any $i \in \mathcal{R}$. Together with $(99)$ and $(101)$, this yields

$$
\begin{aligned}
\sum_{i \in \mathcal{D}\left(\mathcal{B}^{\prime}\right)}\left(g^{i}\left(\mathcal{B}^{\prime}\right)-g^{i}(\mathcal{B})\right) & =\sum_{i \in \mathcal{D}\left(\mathcal{B}^{\prime}\right)}\left(p^{i}(\mathcal{B})-p^{i}\left(\mathcal{B}^{\prime}\right)\right)+\sum_{i \in \mathcal{R}}\left(\delta^{i}\left(\mathcal{B}^{\prime}\right)-S^{i}\left(\mathcal{B}^{\prime}\right)\right)+\sum_{i \in \mathcal{D}(\mathcal{B})}\left(\min \left(\beta \zeta^{i}, \delta^{i}\left(\mathcal{B}^{\prime}\right)\right)-\zeta^{i}\right) \\
& =\sum_{i \in \mathcal{S}\left(\mathcal{B}^{\prime}\right)} \zeta^{i}+\sum_{i \in \mathcal{R}}\left(\delta^{i}\left(\mathcal{B}^{\prime}\right)-S^{i}\left(\mathcal{B}^{\prime}\right)\right)+\sum_{i \in \mathcal{D}(\mathcal{B})} \min \left(\beta \zeta^{i}, \delta^{i}\left(\mathcal{B}^{\prime}\right)\right),
\end{aligned}
$$

where we have used that

$$
\sum_{i \in \mathcal{D}\left(\mathcal{B}^{\prime}\right)}\left(p^{i}(\mathcal{B})-p^{i}\left(\mathcal{B}^{\prime}\right)\right)=\sum_{i=1}^{n}\left(p^{i}(\mathcal{B})-p^{i}\left(\mathcal{B}^{\prime}\right)\right)=\sum_{i=1}^{n} \zeta^{i}
$$

since $\pi$ is column-stochastic. It now follows from (98) and 102 that

$$
\begin{aligned}
W_{\lambda}\left(s\left(\mathcal{B}^{\prime}\right)\right)-W_{\lambda}(s(\mathcal{B}))= & (1+\lambda) \sum_{i \in \mathcal{S}(\mathcal{B})} \zeta^{i}-\lambda \sum_{i \in \mathcal{S}(\mathcal{B})} \min \left(\zeta^{i}, C^{i}(\mathcal{B})\right)-(1+\lambda) \sum_{i \in \mathcal{R}} S^{i}\left(\mathcal{B}^{\prime}\right) \\
& +(1+\lambda) \sum_{i \in \mathcal{R}} \delta^{i}\left(\mathcal{B}^{\prime}\right)+(1+\lambda) \sum_{i \in \mathcal{D}(\mathcal{B})} \min \left(\beta \zeta^{i}\left(\mathcal{B}^{\prime}\right), \delta^{i}\left(\mathcal{B}^{\prime}\right)\right)
\end{aligned}
$$

which readily implies 22 .

Proof of Lemma 5.5. Fix a set $\mathcal{B}_{*} \in \mathcal{B}_{P}$ of banks to be rescued in the threatened bailout. Fix two sets of banks $\mathcal{B}^{\prime} \subseteq \mathcal{B}$ and let $\zeta=\pi\left(p(\mathcal{B})-p\left(\mathcal{B}^{\prime}\right)\right)$. Observe that $\zeta \geq 0$ since $p(\cdot)$ is monotonic in the set of rescued banks. It follows in the same way as in the proof of Lemma 5.2 that $C\left(\mathcal{B}^{\prime}\right)=$ $(C(\mathcal{B})-\zeta)^{+}$. Together with the definition of $\eta(\mathcal{B})$ in $(23)$, this implies

$$
\eta\left(\mathcal{B}^{\prime}\right)=\min \left(\left(\pi\left(p(\mathcal{B})-p\left(\mathcal{B}_{*}\right)\right)-s\left(\mathcal{B}_{*}\right)-\zeta\right)^{+}, C\left(\mathcal{B}^{\prime}\right)\right)=(\eta(\mathcal{B})-\zeta)^{+}
$$

It now follows from the elementary identity $a-(a-b)^{+}=\min (a, b)$ that

$$
\eta(\mathcal{B})-\eta\left(\mathcal{B}^{\prime}\right)=\min (\zeta, \eta(\mathcal{B}))
$$


Together with (103), this implies the statement.

Proof of Lemma 5.6. Fix $\mathcal{B}_{*} \in \mathcal{B}_{P}$, from which the threats are chosen. Fix two sets $\mathcal{B} \subseteq \mathcal{B}^{\prime}$ and note that $\eta\left(\mathcal{B}^{\prime}\right) \geq \eta(\mathcal{B})$ by $(104)$. It follows that

$$
W_{\lambda}\left(s\left(\mathcal{B}^{\prime}\right)\right)-\lambda \sum_{j=1}^{m(\mathcal{B})} \eta^{i_{j}(\mathcal{B})}\left(\mathcal{B}^{\prime}\right) \leq W_{\lambda}(s(\mathcal{B}))-\lambda \sum_{j=1}^{m(\mathcal{B})} \eta^{i_{j}(\mathcal{B})}(\mathcal{B})<W_{P}^{*}
$$

Because $m^{\prime}(\mathcal{B})$ is the size of the smallest set of contributors to attain welfare losses lower than $W_{P}^{*}$ with contributions $\eta\left(\mathcal{B}^{\prime}\right)$, this shows that $m\left(\mathcal{B}^{\prime}\right) \leq m(\mathcal{B})$. Since $\eta^{i}\left(\mathcal{B}^{\prime}\right) \geq \eta^{i}(\mathcal{B})$ for any bank $i$, the $k^{\text {th }}$-largest element in $\eta\left(\mathcal{B}^{\prime}\right)$ must be at least as large as the $k^{\text {th }}$-largest element in $\eta(\mathcal{B})$ for any $k$. This shows that

$$
\begin{aligned}
W_{B_{*}}\left(\mathcal{B}^{\prime}\right) & \leq W_{P}^{*}-\lambda \eta^{i_{m\left(\mathcal{B}^{\prime}\right)+1}\left(\mathcal{B}^{\prime}\right)}\left(\mathcal{B}^{\prime}\right) \\
& \leq W_{P}^{*}-\lambda \eta^{i_{m\left(\mathcal{B}^{\prime}\right)+1}(\mathcal{B})}(\mathcal{B}) \leq W_{P}^{*}-\lambda \eta^{i_{m(\mathcal{B})+1}(\mathcal{B})}(\mathcal{B})
\end{aligned}
$$

where we used $m\left(\mathcal{B}^{\prime}\right) \leq m(\mathcal{B})$ in the last inequality. To show $W_{\mathcal{B}_{*}}\left(\mathcal{B}^{\prime}\right) \leq W_{\mathcal{B}_{*}}\left(\mathcal{B}^{\prime}\right)$, we distinguish two cases. Consider first the case when $m\left(\mathcal{B}^{\prime}\right)=m(\mathcal{B})$. Then

$$
W_{\mathcal{B}_{*}}\left(\mathcal{B}^{\prime}\right) \leq W_{\lambda}\left(s\left(\mathcal{B}^{\prime}\right)\right)-\lambda \sum_{i=1}^{m(\mathcal{B})} \eta^{i_{j}\left(\mathcal{B}^{\prime}\right)}\left(\mathcal{B}^{\prime}\right) \leq W_{\lambda}(s(\mathcal{B}))-\lambda \sum_{i=1}^{m(\mathcal{B})} \eta^{i_{j}(\mathcal{B})}(\mathcal{B}) .
$$

Together with (105), this implies $W_{\mathcal{B}_{*}}\left(\mathcal{B}^{\prime}\right) \leq W_{\mathcal{B}_{*}}(\mathcal{B})$. If $m\left(\mathcal{B}^{\prime}\right)<m(\mathcal{B})$, then

$$
\begin{aligned}
W_{\mathcal{B}_{*}}\left(\mathcal{B}^{\prime}\right) & \leq W_{P}^{*}-\lambda \eta^{i_{m\left(\mathcal{B}^{\prime}\right)+1}\left(\mathcal{B}^{\prime}\right)}\left(\mathcal{B}^{\prime}\right) \\
& \leq W_{P}^{*}-\lambda \eta^{i_{m(\mathcal{B})}(\mathcal{B})}(\mathcal{B}) \leq W_{\lambda}(s(\mathcal{B}))-\lambda \sum_{i=1}^{m(\mathcal{B})} \eta^{i_{j}(\mathcal{B})}(\mathcal{B})
\end{aligned}
$$

where we have used the fact that $W_{P}^{*} \leq W_{\lambda}(s(\mathcal{B}))-\lambda \sum_{i=1}^{m(\mathcal{B})-1} \eta^{i_{j}(\mathcal{B})}(\mathcal{B})$ by definition of $m(\mathcal{B})$. Together with 105 , this shows again that $W_{\mathcal{B}_{*}}\left(\mathcal{B}^{\prime}\right) \leq W_{\mathcal{B}_{*}}(\mathcal{B})$.

\section{I.3 Counterexample}

Consider a network consisting of $n=4$ banks with assets and liabilities given as in the left panel of Figure 1 and the interbank network given in the right panel of Figure 1. Let further $\gamma=0, \beta=0.4$, and $\lambda=0.5$. Banks 1 and 2 are fundamentally defaulting banks. In absence of intervention, they cannot repay what they owe to banks 3 and 4 . This causes the contagious default of bank 3 because it becomes unable to repay its senior creditors. The clearing payment vector is $p_{N}=(0.4,0.4,0,0)$ and welfare losses without intervention are equal to 9.824 .

A large part of those welfare losses come from the default of bank 3 because of its large asset value. Solvency of bank 3 can be established by either rescuing bank 1 or bank 2 . Because the 


\begin{tabular}{c|ccc} 
Bank & $L^{i}$ & $w^{i}$ & $c^{i}$ \\
\hline 1 & 2.9 & 0 & 1 \\
2 & 3 & 0 & 1 \\
3 & 0 & 10 & 8.5 \\
4 & 0 & 0 & 1
\end{tabular}

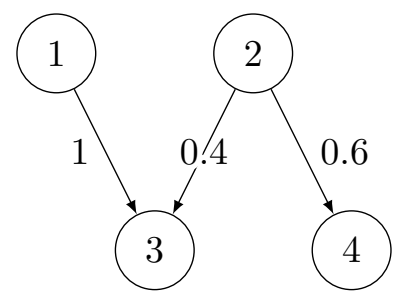

Figure 1: Example of a network with $n=4$ banks, in which the equilbirium partial bail-in does not rescue all the banks that are bailed out in the optimal partial bailout.

liabilities and hence the shortfall of bank 1 are slightly smaller than those of bank 2 , the optimal bailout rescues bank 1 by awarding subsidies $s=(1.9,0,0,0)$. This guarantees solvency of banks 1 , 3 , and 4 and leads to welfare losses $W_{P}^{*}=1.55$.

For the optimal bail-in, additional considerations are taken into account. The bail-in that provides subsidies only to bank 1 leads to the same clearing payment vector as the optimal bailout. It follows from Lemma 5.3 that the threat level towards all banks is 0 and, thus, that bail-in coincides with the optimal bailout. In comparison, the bail-in that rescues bank 2 provides benefits to bank 4 that id not present in the optimal bailout. Thus, bank 4 has an incentive to participate in the bail-in up to a contribution of size $b^{4}=1.56$. The corresponding bail-in leads to welfare losses of $W_{\lambda}(\{2\})=0.82$. Finally, the bail-in that rescues both banks 1 and 2 allows the regulator to extract contributions from both banks 3 and 4 . However, the additional contribution from bank 3 is not large enough to make up for the additional subsidies paid because the large default costs of bank 3 are already prevented by the rescue of bank 2 . Indeed, the corresponding welfare losses are equal to $W_{\lambda}(\{1,2\})=1.03$, which shows that the optimal bail-in may not save banks that are protected in the optimal bailout. 